JOURNAL OF

SYMPLECTIC GEOMETRY

Volume 9, Number 4, 483-636, 2011

\title{
FLOER TRAJECTORIES WITH IMMERSED NODES AND SCALE-DEPENDENT GLUING
}

\author{
Yong-Geun OH AND Ke Zhu
}

Development of pseudo-holomorphic curves and Floer homology in symplectic topology has led to moduli spaces of pseudo-holomorphic curves consisting of both "smooth elements" and "spiked elements", where the latter are combinations of $\boldsymbol{J}$-holomorphic curves (or Floer trajectories) and gradient flow line segments. In many cases the "spiked elements" naturally arise under adiabatic degeneration of "smooth elements" which gradually go through thick-thin decomposition. The reversed process, the recovering problem of the "smooth elements" from "spiked elements" is recently of much interest.

In this paper, we define an enhanced compactification of the moduli space of Floer trajectories under Morse background using the adiabatic degeneration and the scale-dependent gluing techniques. The compactification reflects the one-jet datum of the smooth Floer trajectories nearby the limiting nodal Floer trajectories arising from adiabatic degeneration of the background Morse function. This paper studies the gluing problem when the limiting gradient trajectories has length zero through a renomalization process. The case with limiting gradient trajectories of nonzero length will be treated elsewhere.

An immediate application of our result is a complete proof of the isomorphism property of the PSS map: a proof of this isomorphism property was outlined by Piunikhin-Salamon-Schwarz [PSS] in a way somewhat different from the current proof in its details. This kind of scale-dependent gluing techniques was initiated in [FOOO2] in relation to the metamorphosis of holomorphic polygons under Lagrangian surgery and is expected to appear in other gluing and compactification problem of pseudo-holomorphic curves that involves 'adiabatic' parameters or rescaling of the targets.

Contents

1. Introduction

1.1. Adiabatic degeneration of Floer trajectories 
1.2. Nodal Floer trajectories with immersed nodes $\quad 489$

1.3. Related works and organization of the content 491

2. Review of the classical Floer's equation $\quad 492$

3. Invariant setup of the Floer equation 493

3.1. Punctures with analytic coordinates $\quad 494$

3.2. Hamiltonian perturbations $\quad 494$

3.3. Floer moduli spaces $\quad 496$

4. Formulation of the PSS maps $\quad 499$

4.1. The smooth moduli space $\mathcal{M}_{\left(s_{0} ; s_{+}, s_{-}\right)}(K, J ; B) \quad 499$

4.2. The PSS maps $\Phi$ and $\Psi \quad 500$

4.3. The PSS-scheme of proof of the isomorphism property $\Phi_{*} \quad 503$

Part I. Geometry: Floer trajectories with immersed nodes 505

5. Definition of the deformation-cobordism moduli space 505

5.1. Moduli space of "disk-flow-disk" configurations 505

5.2. Nodal Floer trajectories of PSS deformation at $\lambda=0$

512

5.3. Moduli space of enhanced nodal Floer trajectories 513

$\begin{array}{ll}\text { 5.4. Nodal points are immersed } & 517\end{array}$

5.5. Resolved nodal Floer trajectories in Morse background $\quad \mathbf{5 2 2}$

6. Local models near nodes in vacuum $\quad 524$

6.1. Classification of local models $\quad 524$

6.2. Fredholm theory of local models $\quad 528$

6.3. Transversality of local models 531

7. Local models near nodes in Morse background $\quad 534$

8. Off-shell framework for the gluing $\quad 540$

8.1. Darboux family and explosion of manifolds 541

8.2. Off-shell formulation for perturbed $J$-holomorphic discs

8.3. Off-shell formulation of enhanced nodal Floer trajectories

553

8.4. Appendix: Explosion $\quad 557$

Part II. Analysis: scale-dependent gluing and compactification

9. Smoothing of nodal Floer trajectories I; to "disk-flowdisk" 
10. Smoothing of nodal Floer trajectories II; to Floer trajectories

10.1. Construction of approximate solutions

10.2. Error estimates of approximate solutions

10.3. The off-shell setting of resolved nodal Floer trajectories

10.4. Construction and estimates of the right inverse

12. Controlled nodal degeneration of Floer trajectories

12.1. Convergence in level 0: stable map convergence

12.2. One-jet convergence to nodal curves with immersed nodes

13. Surjectivity of the scale-dependent gluing family

603

13.1. Convergence in the neck regions

605

13.2. Convergence in the central region

610

Part III. Application: a proof of PSS isomorphism

619

14. Review of Floer complex and operators

620

15. $\Psi \circ \Phi=\mathrm{i} d$; Floer via Morse back to Floer

627

16. $\Phi \circ \Psi=\mathrm{i} d$; Morse via Floer back to Morse

629

Acknowledgments

632

References

632

Index

635

\section{Introduction}

Development of pseudo-holomorphic curves and Floer homology in symplectic geometry has led to moduli spaces consisting of both "smooth elements" and "spiked elements", where the latter are combinations of $\boldsymbol{J}$ holomorphic curves (or Floer trajectories) and gradient flow segments. For example, they appear in the generalized holomorphic building in symplectic field theory [BEHWZ], in the quantum structure and cluster complex for Lagrangian submanifolds $[\mathbf{B C}, \mathbf{C L}]$, and even earlier in the works $[\mathbf{F u 2 ,}$ Oh1, PSS, Sc1, MT]. In many cases the "spiked elements" naturally arise from adiabatic degeneration of "smooth elements" which gradually decompose into "thick parts" and "thin parts".

The adiabatic degeneration and its reversal process of the type studied in this paper has appeared in $[\mathbf{F O h}, \mathbf{E k}, \mathbf{R}]$. The paper $[\mathbf{S W}]$ studied another type of adiabatic degeneration in a different context. All these papers are, however, restricted to the case without quantum contribution, i.e, without bubbling phenomenon. The papers $[\mathbf{O h} 5, \mathbf{O h 3}, \mathbf{M T}]$ studied 
adiabatic degeneration with quantum contribution close to the one studied in this paper. However, the recovering problem was only mentioned and left as a future work in $[\mathbf{O h} 1, \mathbf{M T}]$.

Part of the difficulty for the recovering problem lies in finding good local models near the junction points where the $\boldsymbol{J}$-holomorphic curve and gradient flow intersect. It turns out the derivative information of the $\boldsymbol{J}$-holomorphic curve and the gradient flow at the junction point is needed to determine the local model. Besides a good local model, appropriate Fredholm theory and implicit function theorem are needed in order to glue the "spiked elements" to "smooth elements" in a controlled way to reflect the adiabatic degeneration. It turns out that the scale-dependent gluing technique carried out in chapter 10 [FOOO2] of [FOOO1] in relation to metamorphosis of $\boldsymbol{J}$-holomorphic polygon under the Lagrangian surgery, which treats a small region near the junction point as about the same size as the original target manifold, is needed to retain the geometric features of the local model under the perturbation via implicit function theorem. Large part of the analysis used in this paper is motivated by those in [FOOO2].

1.1. Adiabatic degeneration of Floer trajectories. In this paper, we study the adiabatic degeneration of maps $u: \mathbb{R} \times S^{1} \rightarrow M$ satisfying the following one-parameter $\left(0<\varepsilon<\varepsilon_{0}\right)$ family of Floer equations:

$$
\left(d u+P_{K_{\varepsilon}}(u)\right)_{J_{\varepsilon}}^{(0,1)}=0 \quad \text { or equivalently } \bar{\partial}_{J_{\varepsilon}}(u)+\left(P_{K_{\varepsilon}}\right)_{J_{\varepsilon}}^{(0,1)}(u)=0,
$$

We refer to Section 3 for detailed exposition of (1.1), the invariant form of the Floer equation. The expression of the degenerating Hamiltonian $K_{\varepsilon}$ : $\mathbb{R} \times S^{1} \times M \rightarrow \mathbb{R}$ is given by

$$
K_{\varepsilon}(\tau, t, x)= \begin{cases}\kappa_{\varepsilon}^{+}(\tau) \cdot H(t, x), & \text { for } \tau \geq R(\varepsilon) \\ \rho_{\varepsilon}(\tau) \cdot \varepsilon f(x), & \text { for }|\tau| \leq R(\varepsilon) \\ \kappa_{\varepsilon}^{-}(\tau) \cdot H(t, x), & \text { for } \tau \leq-R(\varepsilon)\end{cases}
$$

where $\kappa_{e}^{ \pm}$and $\rho_{\varepsilon}$ are suitable cut-off functions (see (5.34) for the precise definition). This type of equations, for example, appears in the study of isomorphism property of the PSS map introduced in [PSS].

Roughly speaking, the adiabatic degeneration occurs because $K_{\varepsilon}$ restricts to Morse function $\varepsilon f$ on longer and longer cylinder $[-R(\varepsilon), R(\varepsilon)] \times S^{1}$ in $\mathbb{R} \times S^{1}$. A basic assumption that we put on this paper is that $R(\varepsilon)$ satisfies

$$
\lim _{\varepsilon \rightarrow 0} \varepsilon R(\varepsilon)=0 \text {. }
$$

The general case of $\lim _{\varepsilon \rightarrow 0} \varepsilon R(\varepsilon)=\ell$ for $\ell>0$ will be studied in a forthcoming paper [OZ2]. Under this assumption, it is proved in [Oh5, Oh3, MT] that as $\varepsilon \rightarrow 0$, a degenerating sequence of Floer trajectories converges to a nodal Floer trajectory denoted by $\left(u_{-}, u_{+}\right)$. Descriptions of nodal Floer trajectories and immersed nodal points are now in order. 
Let $\dot{\Sigma}_{+}$be the Riemann sphere with one marked point $o_{+}$and one positive puncture $e_{+}$. Choose analytical charts at $o_{+}$and at $e_{+}$on some neighborhoods $O_{+}$and $E_{+}$, respectively, so that conformally $O_{+} \backslash o_{+} \cong(-\infty, 0] \times S^{1}$, and $E_{+} \backslash e_{+} \cong[0,+\infty) \times S^{1}$. We use $t$ for the $S^{1}$ coordinate and $\tau$ for the $\mathbb{R}$ coordinate. Then $\{-\infty\} \times S^{1}$ and $\{+\infty\} \times S^{1}$ correspond to $o_{+}$and $e_{+}$, respectively.

We consider a vector-valued one-form $K_{+}$on $\dot{\Sigma}$ with its values in the set $\operatorname{ham}(M, \omega)$ of Hamiltonian vector fields on $(M, \omega)$, and $\dot{\Sigma}=\mathbb{C} P^{1} \backslash\left\{e_{+}, o_{+}\right\} \cong$ $\mathbb{C} \backslash\{0\} \cong \mathbb{R} \times S^{1}$. We denote by $(\tau, t)$ the standard coordinate on $\mathbb{R} \times S^{1}$. With respect to this coordinates, we require $K_{+}$satisfy

$$
\begin{cases}K_{+}=0, & \text { near } o_{+} \\ K_{+}=H_{+}(t, x) d t, & \text { near } e_{+}\end{cases}
$$

$H_{+}: S^{1} \times M \rightarrow \mathbb{R}$ is a Hamiltonian function independent of the variable $\tau$.

Let $z_{+}: S^{1} \rightarrow M$ be a nondegenerate periodic orbit of $H_{+}$and consider a finite energy solution $u_{+}: \dot{\Sigma} \rightarrow M$ of the Floer equation (1.1) associated to $K_{+}$. By the finite energy condition and since $K_{+} \equiv 0$ near $o_{+}, u_{+}$extends smoothly across $o_{+}$and can be regarded as a smooth map defined on $\mathbb{C}$ that is holomorphic near the origin $0 \in \mathbb{C}$.

Similarly we consider one-form $K_{-}$on $\dot{\Sigma}$ and $\dot{\Sigma}=\mathbb{C} P^{1} \backslash\left\{o_{-}, e_{-}\right\} \cong$ $\mathbb{C} \backslash\{0\} \cong \mathbb{R} \times S^{1}$. We denote by $(\tau, t)$ the standard coordinate on $\mathbb{R} \times S^{1}$, so that $+\infty$ corresponds to $o_{-}$and $-\infty$ to $e_{-}$. With respect to this coordinates, we require $K_{+}$satisfy

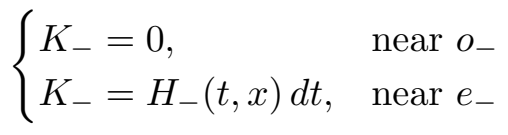

$H_{ \pm}: S^{1} \times M \rightarrow \mathbb{R}$ are a pair of Hamiltonian functions independent of the variable $\tau$. Let $z_{ \pm}: S^{1} \rightarrow M$ be a nondegenerate periodic orbit of $H_{ \pm}$and its lifting $\left[z_{ \pm}, w_{ \pm}\right]$of $z_{ \pm}$.

A nodal Floer trajectory is, by definition, the gluing $u_{-} \# u_{+}$at $u_{-}\left(o_{-}\right)=$ $u_{+}\left(o_{+}\right)$where $u_{ \pm}$are the solutions of the Floer equation associated to $K_{ \pm}$ respectively. We say that a nodal point of $u_{-} \# u_{+}$is immersed if $u_{ \pm}$are immersed at $o_{ \pm}$, respectively.

One of the main results of the paper is the following enhanced compactification theorem in Section 12 (Theorem 12.1) for gluing and its surjectivity:

Theorem 1.1. Suppose that $u_{-}, u_{+}$are immersed at the node

$$
p=u_{-}\left(o_{-}\right)=u_{+}\left(o_{+}\right) .
$$

Let Glue $\left(u_{-}, u_{+}\right)$be the nodal Floer trajectory formed by $u_{-}$and $u_{+}$ with nodal points $p=u_{-}(o-)=u_{+}\left(o_{+}\right)$. Suppose that $u_{n}$ converges to Glue $\left(u_{-}, u_{+}\right)$in level 0 . Then there exists a subsequence $u_{n_{i}}$ and a sequence $\varepsilon_{i} \rightarrow 0$ such that $u_{n_{i}}$ converges to $\left(u_{-}, u_{+}, u_{0}\right)$ in the $\left\{\varepsilon_{i}\right\}$-controlled way. 
A more detailed description of the local model $u_{0}$ above is in order now.

The convergence in level 0 is the usual Gromov convergence (Definition 12.3). The convergence in $\varepsilon$-controlled way is in Definition 12.4. Roughly speaking, we magnify suitable small neighborhood of the center of the neck of $u_{n_{i}}$ to keep track of the degeneration in microscopic level, and in the limit we get a proper holomorphic curve in $\mathbb{C}^{n}$ with asymptotic convergence to simple Reeb orbits $\gamma_{-}$and $\gamma_{+}$of the standard contact form $\lambda$ on $S^{2 n-1}$ in the cylindrical end $\mathbb{R} \times\left(S^{2 n-1}, \lambda\right)$ of $\mathbb{C}^{n}$. Such a holomorphic curve can be identified as a degree 2 rational curve in $\mathbb{C} P^{n}$ intersecting the hyperplane at infinity at two points $x_{0}$ and $x_{\infty}$. We have the following classification result of such rational curves (Proposition 6.1):

Proposition 1.1. Fix a hyperplane $H$ in $\mathbb{C} P^{n}$ and two points $x_{0}, x_{\infty} \in H$. Then there exists a unique rational curve passing through $x_{0}, x_{1}$ and of degree 2 modulo the action of $A u t\left(\mathbb{C} P^{n} ; H\right)$, which is the group of automorphisms of $\mathbb{C} P^{n}$ fixing $H$.

We can give an explicit formula for such rational curves. (See Remark 6.1 for such formula.) We refer to Theorem 6.1 for the explanation how this proposition can be used to provide the local model $u_{0}$. (Strictly speaking the microscopic adiabatic limit has some remnant from the background small Morse function $\varepsilon f$ put in the middle of $K_{\varepsilon}$ (5.34) above and is a proper holomorphic curve perturbed by a linear vector $\tau \nabla f(p)$, i.e., has the form $u_{0}+\tau \nabla f(p)$ See Proposition 7.2.)

We call $\left(u_{-}, u_{+}, u_{0}\right)$ an enhanced nodal Floer trajectory. For a given enhanced nodal Floer trajectory, we glue a 1-parameter family of smooth Floer trajectories and show that they are all possible nearby smooth Floer trajectories according to the topology defined by the above enhanced convergence. (See Theorem 10.1 for the precise statement.)

Equation (1.1) is nothing but a coordinate free expression of the equation arising in the framework of the PSS map described in [PSS]. A key step during the PSS scheme of proof of the isomorphism property is to resolve the nodal Floer trajectory to a 1-parameter family of smooth Floer trajectories. Unlike the smoothing trajectories obtained via the more conventional gluing outlined in [PSS, MS] (see also $[\mathbf{L u}]$ ), our resolved Floer trajectories is more closely tied to the limiting configurations arising through adiabatic degeneration in that they are aligned in the gradient flow direction near the node and is related to the disk-flow-disk elements.

Remark 1.1. As far as we know, the detail of a key gluing result needed in the proof of isomorphism property of the PSS map announced in [PSS] has not been given yet in any previous literature and our paper is the first one that provides a full detail of the proof of isomorphism property of the PSS map. Our proof uses a somewhat possibly "overkilled" gluing result obtained in Theorems 12.1 and 10.1: Although we did not check it, it is 
conceivable that one might be able to write down a proof, without rescaling target manifolds, following the more standard approach of Floer's gluing [F11]. However we strongly believe that to materialize such a proof one will still need to assume that nodes are immersed as we do in the present paper. Such a requirement has not been addressed in the proposed PSS scheme in $[\mathbf{P S S}]$ or in any other existing literature related to it. We refer to the next subsection for more discussion on the nontriviality of this gluing theorem involved in the PSS scheme. See also Remark 5.2.

In this paper, we take the PSS framework as a test case to apply our scale-dependent gluing scheme thereto because the PSS picture appears as the simplest case for an adiabatic degeneration yet manifests the general technique. Our gluing scheme can also be applied to other context such as in the story told in [Oh1] where the adiabatic degeneration of holomorphic polygons under the total collapse of $k$ Lagrangian graphs Graph $d f_{i}$, $i=1, \ldots, k$ in a Darboux neighborhood of a given Lagrangian submanifold $L \subset M$ was outlined which involves configurations of holomorphic curves joined by gradient trajectories of $k$ different Morse functions in a general symplectic manifold $(M, \omega)$. In this general case there are non-constant holomorphic spheres or discs around unlike the case of cotangent bundle studied in $[\mathbf{F O h}]$. This is a subject of future study [OZ2].

1.2. Nodal Floer trajectories with immersed nodes. Temporarily we denote by $\mathcal{M}_{\varepsilon}$ the general moduli space parameterized by $\varepsilon$ for $-\varepsilon_{0} \leq \varepsilon \leq \varepsilon_{0}$ with some phase change at $\varepsilon=0$. We will focus on the one that appears in the above mentioned PSS scheme but the same story can be applied to more general setting. In relation to the scheme of proof of the isomorphism property of the PSS map, for example, one would like to prove a certain parameterized moduli space

$$
\mathcal{M}^{\text {para }}:=\bigcup_{-\varepsilon_{0} \leq \varepsilon \leq \varepsilon_{0}} \mathcal{M}_{\varepsilon} \rightarrow\left[-\varepsilon_{0}, \varepsilon_{0}\right]
$$

defines a piecewise smooth compact cobordism between $\mathcal{M}_{-\varepsilon_{0}}$ and $\mathcal{M}_{\varepsilon_{0}}$ : there occurs a 'phase change' at $\varepsilon=0$. Due to the 'phase change' at $\varepsilon=0$, one needs to prove a bi-collar theorem of $\mathcal{M}_{0} \subset \mathcal{M}^{\text {para }}$ to materialize the PSS-scheme. From $-\varepsilon_{0}$ to 0, one can construct the left one-sided collar by finite dimensional differential topology (see Section 9). On the other hand, for the right one-sided collar over $\left[0, \varepsilon_{0}\right],[\mathbf{P S S}]$ attempts to produce the collar by a 'standard gluing method' of 'some' perturbed Cauchy Riemann equation. More specifically, $[\mathbf{P S S}]$ attempts to produce a diffeomorphism

$$
\bigcup_{0 \leq \varepsilon \leq \varepsilon_{0}} \mathcal{M}_{\varepsilon} \cong \mathcal{M}_{0} \times\left[0, \varepsilon_{0}\right]
$$


for a sufficiently small $\varepsilon_{0}>0$. However the details of this gluing theorem are given neither in $[\mathbf{P S S}, \mathbf{L u}]$ nor in the recent book $[\mathbf{M S}]$. As far as the authors understand, construction of this diffeomorphism is not as standard as [PSS, MS] indicated.

The main result of the present paper is to construct this one-sided collar, at $\varepsilon=0$ (not at $\infty$ ), by producing a one-parameter family of Floer trajectories out of the nodal Floer trajectories (out of $\mathcal{M}_{0}$ ) by the adiabatic degeneration $[\mathbf{O h} 5, \mathbf{O h} 3, \mathbf{M T}]$ and a scale-dependent gluing method. We would like to emphasize that due to the phase change at $\varepsilon=0$ the standard gluing theorem of parameterized moduli space over $\varepsilon$ cannot be applied either here.

If we only consider the usual stable map convergence a $\varepsilon \rightarrow 0$, we only see the standard nodal Floer trajectories as a degenerate limit when we ignore bubbling-off-spheres. But to recover the nearby resolved Floer trajectories for $\varepsilon>0$ and construct the above mentioned one-sided collar, we need extra one-jet data that is lost into the node during the standard stable map convergence. For this purpose, it is essential to assume that nodal points are immersed. For the purpose of completing the proof of isomorphism property of the PSS map, consideration of such nodal Floer trajectories will be sufficient.

Let $\left[z_{ \pm}, w_{ \pm}\right]$be periodic orbits with caps of $H_{ \pm}$, respectively. We denote by

$$
\mathcal{M}_{\text {stand }}^{\text {nodal }}\left(\left[z_{-}, w_{-}\right],\left[z_{+}, w_{+}\right] ;(K, J)\right)
$$

the set of nodal Floer trajecotories in class $B \in \pi_{2}\left(z_{-}, z_{+}\right)$that satisfies

$$
\left[w_{-}\right] \# B \#\left[w_{+}\right]=0 .
$$

Here $\pi_{2}\left(z_{-}, z_{+}\right)$is the set of homotopy class of maps $w:[0,1] \times S^{1} \rightarrow M$ satisfying $w(0, t)=z_{-}(t), w(1, t)=z_{+}(t)$. Note that the gluing $u_{-} \# u_{+}$canonically assigns a class in $\pi_{2}\left(z_{-}, z_{+}\right)$. A general index theorem [SZ] says that the virtual dimension of the moduli space $\mathcal{M}_{\text {stand }}^{\text {nodal }}\left(\left[z_{-}, w_{-}\right],\left[z_{+}, w_{+}\right] ;(K, J)\right)$ is given by

$$
\mu_{H_{-}}\left(\left[z_{-}, w_{-}\right]\right)-\mu_{H_{+}}\left(\left[z_{+}, w_{+}\right]\right)
$$

where $\mu_{H}([z, w])$ is the Conley-Zehnder index $[\mathbf{C Z}]$ of the periodic orbit $z$ with cap $w$ associated to the Hamiltonian $H$. The sign conventions of $[\mathbf{C Z}, \mathbf{S Z}]$ are different from those used in the present paper one way or the other. We refer to Appendix of [Oh4] for a discussion of the index formula in the convention used in the present paper.

The following theorem enable us to consider only the nodal Floer trajectories with immersed node for the purpose of proving isomorphism property of the PSS map. 
Theorem 1.2. Let $\left(K_{ \pm}, J_{ \pm}\right)$be a Floer datum with the asymptotic Hamiltonian $H_{ \pm}$. Suppose that

$$
\mu_{H_{-}}\left(\left[z_{-}, w_{-}\right]\right)-\mu_{H_{+}}\left(\left[z_{+}, w_{+}\right]\right)<2 n-1 .
$$

Then there exists a dense subset of $\mathcal{J}_{\omega}$ consisting of $J$ 's such that for any quadruple

$$
\left(u_{-}, u_{+}, r_{-}, r_{+}\right) \in \mathcal{M}_{\text {stand }}^{\text {nodal }}\left(\left[z_{-}, w_{-}\right],\left[z_{+}, w_{+}\right] ;(K, J)\right)
$$

with $u_{-}\left(r_{-}\right)=u_{+}\left(r_{+}\right), r_{-}$and $r_{+}$are immersed points of $u_{-}$and $u_{+}$respectively, and

$$
\left[d u_{-}\left(r_{-}\right)\right] \neq\left[d u_{+}\left(r_{+}\right)\right] \quad \text { in } \mathbb{P}\left(T_{x} M\right)
$$

with $x=u_{-}\left(r_{-}\right)=u_{+}\left(r_{+}\right)$. The same holds for a one parameter family of $\operatorname{such}\left(K_{ \pm}, J_{ \pm}\right)$.

In particular, these hold when $\mu\left(\left[z_{-}, w_{-}\right]\right)-\mu\left(\left[z_{+}, w_{+}\right]\right)=0$, or -1 .

1.3. Related works and organization of the content. Our gluing theorem involves two moduli spaces in different scales. This kind of gluing theorem first appeared in $[\mathbf{F O h}]$ in symplectic geometry, in which Fukaya and Oh glued holomorphic discs with boundary punctures at the intersections of several gradient trajectories of different Morse functions after they are shrunk with a prescribed scale depending on the degeneration parameter $\varepsilon$. Another scale-dependent gluing theorem has been also used in [FOOO2] in relation to the Lagrangian surgery and metamorphosis of holomorphic polygons. Furthermore the kind of analysis that has been used for the analysis of proper pseudo-holomorphic curves in symplectic manifolds with cylindrical ends [Ho, HWZ1, HWZ2, HWZ4] also plays a crucial role in our analysis. This analysis is further complicated by the fact that we have to work out the relevant estimates in the setting of asymptotically cylindrical ends on incomplete manifolds, especially in the proof of surjectivity of the gluing.

Finally it would be worthwhile to mention that the analysis given in the present paper is a first step towards a full understanding of the conjectural picture described in $[\mathbf{O h} \mathbf{1}]$, which would require this type of scale-dependent gluing theorem of pseudo-holomorphic curves under the background Morse function, or twisted by the Hamiltonian flow of a Morse function. Based on the argument of adiabatic degeneration, the senior author indicated that 'homology' of the quantum chain complex will be isomorphic to that of the Floer complex, if they defined. Study of some related collapsing degenerations has been carried out by the senior author in $[\mathbf{O h} 5, \mathbf{O h 3}]$ and by Mundet i Rierra and Tian [MT].

We would like to mention one potential application of our gluing scheme. In [FOOO2], scale-dependent gluing was used to compare the moduli space of $\boldsymbol{J}$-holomorphic triangles ending on 3 Lagrangian submanifolds $\left(L_{0}, L_{1}, L_{2}\right)$ and the moduli space of $\boldsymbol{J}$-holomorphic 2 -gons ending on two Lagrangian submanifolds $\left(L_{0}, L_{1} \#_{\lambda} L_{2}\right)$, where $L_{1} \#_{\lambda} L_{2}$ is obtained by 
Lagrangian surgery from $L_{1}$ and $L_{2}$. Similar to the Lagrangian surgery to smooth the singular Lagrangian submanifolds $L_{1} \cup L_{2}$ to $L_{1} \#_{\lambda} L_{2}$, we expect our scale-dependent gluing can be used to understand $\boldsymbol{J}$-holomorphic curves in singular target spaces, or its change when the target manifold undergoes some surgery.

A brief summary of each part of the paper is in order. In Part I, we set up a new geometric framework which addresses an enhancement of the description of standard nodal Floer trajectories. In this enhancement, it is essential to assume that the nodes of nodal Floer trajectories are immersed and to insert suitable local models at the nodes in one-jet level, so we prove Theorem 1.2 (Theorem 5.1).

In Part II, we carry out a scale-dependent gluing analysis to glue two outer pseudo-holomorphic curves and the local model in different scale which is somewhat reminiscent of the ones in [FOh,FOOO2]. In this scaledependent analysis, the immersion property of nodal points and a proper choice of scales of neck-stretching relative to the adiabatic parameter is essential.

In Part III, we combine these with the standard deformation-cobordism argument to explain how our gluing theorem can be used to give a proof of the isomorphism property of the PSS map.

\section{Review of the classical Floer's equation}

Throughout this paper, $(M, \omega)$ is a compact symplectic manifold. We will always identify $S^{1}$ with $\mathbb{R} / \mathbb{Z}$, which in particular has the canonical marking $0(\bmod 1) \in S^{1}$. Denote by $S=\mathbb{R} \times S^{1}$ the infinite cylinder with the unique complex structure, denoted by $j$. We denote by $(\tau, t)$ be the associated cylindrical coordinates such that

$$
\tau+\mathrm{i} t, \quad \tau \in \mathbb{R}, \quad t \in S^{1}=\mathbb{R} / \mathbb{Z}
$$

provides the standard complex coordinates on $S$ identified with the quotient space $S=\mathbb{C} / i \mathbb{Z}$ which lifts to the standard coordinates $z=\mathrm{e}^{2 \pi(\tau+\mathrm{i} t)}$ on $\mathbb{C}$.

Let $J=J(\tau, t)$ be a two-parameter family of almost complex structures compatible with $\omega$ for $(\tau, t) \in R \times S^{1}$ satisfying the asymptotic condition

$$
J(\tau, t) \equiv J( \pm \infty, t), \quad \text { for } \tau \geq R_{+} \quad \text { and } \quad \tau \leq-R_{-}
$$

for some $R_{ \pm} \geq 0$. Denote the set of all such $J$ by $\mathcal{J}=\mathcal{J}_{\omega}$, and by $\mathcal{J}_{\omega}^{\text {cyl }}$ the set of such $J$ 's independent of $\tau$.

Next, we consider two parameter family of smooth functions on $M$ parameterized by $(\tau, t) \in \mathbb{R} \times S^{1}$

$$
H=H(\tau, t, x)
$$

such that $H(\tau, t, x) \equiv H_{ \pm}(t, x)$ for $\tau \geq R_{+}$or $\tau \leq R^{-}$. We call $H$ cylindrical if $H$ is independent of $\tau$. For each given cylindrical $H$, we consider the 
Hamilton equation

$$
\dot{x}=X_{\mathrm{H}}(t, x), t \in S^{1},
$$

where $X_{\mathrm{H}}$ is the Hamiltonian vector field of $H$, and denote by $\operatorname{Per} H$ the set of one-periodic solutions $z(t)$, i.e., those satisfying $z(0)=z(1)$. We note that $z(t)$ can be written as $z(t)=\phi_{\mathrm{H}}^{t}(x)$ for some $x \in M$, where $\phi_{H}^{t}$ is the Hamiltonian flow for $H$ at time $t$. Then $z(t)$ is periodic if and only if $x$ is a fixed point of the time-one map $\phi_{\mathrm{H}}^{1}$ of $X_{\mathrm{H}}$.

For each given periodic orbits $z_{ \pm}(t)$ at $\tau= \pm \infty$ of $H_{ \pm}$, respectively, the Floer's perturbed Cauchy Riemann equation associated to the pair $(H, J)$ has the form

$$
\left\{\begin{array}{l}
\frac{\partial u}{\partial \tau}+J\left(\frac{\partial u}{\partial t}-X_{H}(u)\right)=0 \\
u(-\infty, t)=z_{-}(t), u(\infty, t)=z_{+}(t)
\end{array}\right.
$$

for a map $u: \mathbb{R} \times S^{1} \rightarrow M$. We call this equation Floer's perturbed CauchyRiemann equation or simply as the perturbed Cauchy-Riemann equation (associated to the pair $(H, J)$ ). This equation may be regarded as the negative gradient flow equation of an action functional defined on the Novikov covering space. The Floer theory largely relies on the study of the moduli spaces of finite energy solutions $u: \mathbb{R} \times S^{1} \rightarrow M$ of the kind (14.5). The relevant energy function is given by

Definition 2.1. For a given smooth map $u: \mathbb{R} \times S^{1} \rightarrow M$, we define the energy, denoted by $E_{(H, J)}(u)$, of $u$ by

$$
E_{(H, J)}(u)=\frac{1}{2} \int\left(\left|\frac{\partial u}{\partial \tau}\right|_{J_{t}}^{2}+\left|\frac{\partial u}{\partial t}-X_{H}(u)\right|_{J_{t}}^{2}\right) d t d \tau .
$$

Equation (14.5) has translational symmetry for the cylindrical pair $(H, J)$ and counting the isolated trajectories of such pair defines the Floer boundary map, and counting isolated trajectories of generic (noncylindrical) pair defines the Floer chain map. This finishes the summary of Floer's original setup of the Floer homology.

When one considers the product structure on the Floer homology, one needs to consider general Riemann surfaces, $\dot{\Sigma}$ of genus zero with punctures. We denote by $\Sigma$ a closed Riemann surface, possibly with nonempty boundary $\partial \Sigma$, and $\dot{\Sigma}$ the corresponding punctured Riemann surface with a finite number of marked points in Int $\Sigma$.

\section{Invariant setup of the Floer equation}

In this section, we will formulate the setup for the general Floer's perturbed Cauchy-Riemann equation on compact Riemann surface with a finite number of punctures. This requires a coordinate-free framework of the equation. 
3.1. Punctures with analytic coordinates. We start with the description of positive and negative punctures. Let $\Sigma$ be a compact Riemann surface with a marked point $p \in \Sigma$. Consider the corresponding punctured Riemann surface $\dot{\Sigma}$ with an analytic coordinates $z: D \rightarrow \mathbb{C}$ on a neighborhood $D \subset \dot{\Sigma}$. By composing $z$ with a linear translation of $\mathbb{C}$, we may assume $z(p)=0$.

We know that $D \backslash\{p\}$ is conformally isomorphic to both $[0, \infty) \times S^{1}$ and $(-\infty, 0] \times S^{1}$.

(1) We say that the pair $(p ;(D, z))$ has a incoming cylindrical end (with analytic chart) if $D=z^{-1}\left(D^{2}(1)\right)$ and the chart induces the biholomorphism

$(\tau, t) \in(-\infty, 0] \times S^{1} \mapsto \mathrm{e}^{2 \pi(\tau+\mathrm{i} t)} \in D^{2}(1) \backslash\{0\} \mapsto z^{-1} \in D \backslash\{p\}$.

We call the corresponding puncture $p \in \Sigma$ a positive puncture.

(2) We say that the pair $(p ;(D, z))$ has a outgoing cylindrical end (with analytic chart) if $D=z^{-1}\left(D^{2}(1)\right)$ and the chart $z$ induces the biholomorphism

$(\tau, t) \in[0, \infty) \times S^{1} \mapsto \mathrm{e}^{-2 \pi(\tau+\mathrm{i} t)} \in D^{2}(1) \backslash\{0\} \mapsto z^{-1} \in D \backslash\{p\}$.

In this case, we call the corresponding puncture $(p ;(D, z))$ a negative puncture (with analytic chart).

3.2. Hamiltonian perturbations. Now we describe the Hamiltonian perturbations in a coordinate free fashion. Such a description was given, for example, by Seidel in $[\mathbf{S e 2}, \mathbf{S e 3}, \mathbf{M S}]$.

Let $\Sigma$ be a compact Riemann surface and $\dot{\Sigma}$ denote $\Sigma$ with a finite number of punctures and analytic coordinates. We denote by $\mathcal{J}_{0, \omega}$ the set of almost complex structures that are cylindrical near the puncture with respect to the given analytic charts $z=\mathrm{e}^{ \pm(2 \pi(\tau+\mathrm{i} t)}$. Define $\mathcal{J}_{\Sigma}$ or $\mathcal{J}_{\dot{\Sigma}}$ to be the set of maps $J: \Sigma, \dot{\Sigma} \rightarrow \mathcal{J}_{0, \omega}$, respectively.

We recall that the standard $\bar{\partial}$-operator

$$
\bar{\partial}_{J}: u \mapsto \bar{\partial}_{J} u:=\frac{d u+J \circ d u \circ j}{2}
$$

defines a section of the vector bundle

$$
\Omega_{J}^{(0,1)}(\Sigma, M) \rightarrow C^{\infty}(\Sigma, M)
$$

where the fiber thereof at $u$ is given by the vector space

$$
\Omega_{J}^{(0,1)}\left(u^{*} T M\right):=C^{\infty}\left(\Lambda_{J}^{(0,1)}\left(u^{*} T M\right)\right),
$$

where $\Lambda_{J}^{(0,1)}\left(u^{*} T M\right)$ is the set of anti-J-linear maps from $(T \Sigma, j) \rightarrow(T M, J)$ lifting $u$, or in other words, $u^{*} T M$-valued $(0,1)$-forms on $\Sigma$. Recall we have the decomposition

$$
\Omega^{1}\left(u^{*} T M\right)=\Omega_{J}^{(1,0)}\left(u^{*} T M\right) \oplus \Omega_{J}^{(0,1)}\left(u^{*} T M\right) .
$$


In the cylindrical coordinates $(\tau, t)$, the map

$$
\left.\frac{\partial}{\partial \tau}\right\rfloor(\cdot): \Omega_{J}^{(0,1)}\left(u^{*} T M\right) \rightarrow \Omega^{0}\left(u^{*} T M\right)=C^{\infty}\left(u^{*} T M\right)
$$

defines a local isomorphism and the expression $\frac{\partial u}{\partial \tau}+J \frac{\partial u}{\partial t}$ in the Floer equation is nothing but

$$
2 \bar{\partial}_{J} u\left(\frac{\partial}{\partial \tau}\right) .
$$

We want to regard the perturbation term $-J X_{H}(u)$ in a similar way. It will be the value of the $(0,1)$-part of some one-form $P_{\dot{\Sigma}}(u) \in \Omega^{1}\left(u^{*} T M\right)$. Furthermore, the term involves a Hamiltonian vector field, not a general vector field. We recall the exact sequence

$$
0 \rightarrow \mathbb{R} \rightarrow C^{\infty}(M) \rightarrow \operatorname{ham}(M, \omega) \rightarrow 0,
$$

where $\operatorname{ham}(M, \omega)$ is the set of Hamiltonian vector fields on $(M, \omega)$ and we assume that $M$ is compact and connected. This sequence canonically splits: we have the integration map

$$
\int_{M}: C^{\infty}(M) \rightarrow \mathbb{R} ; h \mapsto \int_{M} h d \mu .
$$

Therefore, this induces a natural exact sequence

$$
0 \rightarrow \Omega^{1}(\Sigma, \mathbb{R}) \rightarrow \Omega^{1}\left(\Sigma, C^{\infty}(M)\right) \rightarrow \Omega^{1}(\Sigma, \operatorname{ham}(M, \omega)) \rightarrow 0 .
$$

If we restrict the Hamiltonians to the mean-normalized ones, i.e., those in the kernel of the above integral map, we have the isomorphism

$$
\Omega^{1}\left(\Sigma, C_{m}^{\infty}(M)\right) \cong \Omega^{1}(\Sigma, \operatorname{ham}(M, \omega)) .
$$

We denote $C_{m}^{\infty}(M)=\operatorname{ker} \int_{M}$.

Now let $K \in \Omega^{1}\left(\Sigma, C^{\infty}(M)\right)$ and denote by $P_{K}$ the corresponding oneform of $\Omega^{1}(\Sigma, \operatorname{ham}(M, \omega))$. Then for each choice of $\xi \in C^{\infty}(T \Sigma), K(\xi)$ gives a function on $M$ and so a Hamiltonian vector field $P_{K}(\xi)=X_{K(\xi)}$ on $M$. In cylindrical coordinate $(\tau, t)$, we want $K$ to satisfy

$$
-2\left(P_{K}\right)^{(0,1)}(u)\left(\frac{\partial}{\partial \tau}\right)=-J X_{H}(u)
$$

It is easy to check that one such choice of $K$ will be

$$
K(\tau, t)=H(t) d t
$$

on the cylindrical ends for an arbitrary choice of $H$.

Definition 3.1. We call $K \in \Omega^{1}\left(\Sigma, C^{\infty}(M)\right)$ cylindrical at the puncture $p \in \Sigma$ with analytic chart $(D, z)$, if it has the form

$$
K(\tau, t)=H(t) d t
$$

in $D \backslash\{p\}$. We denote by $\mathcal{K}_{\dot{\Sigma}}$ the set of such $K^{\prime}$ 's. 
One important quantity associated to the one-form $K$ is a two-form, denoted by $R_{K}$, and defined by

$$
R_{K}\left(\xi_{1}, \xi_{2}\right)=\xi_{1}\left[K\left(\xi_{2}\right)\right]-\xi_{2}\left[K\left(\xi_{1}\right)\right]-\left\{K\left(\xi_{2}\right), K\left(\xi_{1}\right)\right\}
$$

for two vector fields $\xi_{1}, \xi_{2}$, where $\xi_{1}\left[\left(K\left(\xi_{2}\right)\right]\right.$ denotes directional derivative of the function $K\left(\xi_{2}\right)(z, x)$ with respect to the vector field $\xi_{1}$ as a function on $\Sigma$, holding the variable $x \in M$ fixed. It follows from the expression that $R_{K}$ is tensorial on $\Sigma$.

Remark 3.1. This quantity has the interpretation as the curvature of a symplectic vector bundle over $\Sigma$ in the following way [Ba, Se1]. We regard the product $E=\Sigma \times(M, \omega)$ as a bundle of symplectic manifold whose structure group is $\operatorname{Symp}_{0}(M, \omega)$, the identity component of $\operatorname{Symp}(M, \omega)$. Each one-form $K$ defines a horizontal subspace of $T_{(p, x)} E$ given by the subspace

$$
\mathcal{D}_{K}(p, x):=\left\{\left(\xi, X_{K(\xi)}(x)\right) \mid \xi \in T_{p} \Sigma, x \in M\right\}
$$

and so can be regarded as an Ehresmann connection of $T E \rightarrow \Sigma$. Then $R_{K}$ is the corresponding curvature of this connection $K$. Note that the distribution $\mathcal{D}_{K} \subset T E$ is integrable if and only if $R_{K}=0$ and also equivalent to saying that locally $P_{K}$ can be integrated as the two-parameter family of Hamiltonian isotopies

$$
\Lambda:(s, t) \mapsto \phi(s, t) \in \operatorname{Ham}(M, \omega),
$$

where $\operatorname{Ham}(M, \omega)$ is the Hamiltonian diffeomorphism group on $M$. This last statement was essentially proved by Banyaga $[\mathbf{B a}]$. Motivated by this observation, we will call $R_{K}$ as the curvature of the connection $K$.

3.3. Floer moduli spaces. Now we are ready to give the definition of the moduli space of perturbed Cauchy-Riemann equation in a coordinate-free form. The Hamiltonian-perturbed Cauchy-Riemann equation has the form

$$
\left(d u+P_{K}(u)\right)_{J}^{(0,1)}=0 \quad \text { or equivalently } \bar{\partial}_{J}(u)+\left(P_{K}\right)_{J}^{(0,1)}(u)=0
$$

on $\Sigma$ in general. Following Seidel [Se3], we call a pair $(K, J) \in \mathcal{K}_{\dot{\Sigma}} \times \mathcal{J}_{\dot{\Sigma}}$ a Floer datum.

For each given such pair $(K, J)$, it defines a perturbed Cauchy-Riemann operator by

$$
\bar{\partial}_{(K, J)} u:=\bar{\partial}_{J} u+P_{K}(u)_{J}^{(0,1)}=\left(d u+P_{K}(u)\right)_{J}^{(0,1)} .
$$

Let $(\mathfrak{p}, \mathfrak{q})$ be a given set of positive punctures $\mathfrak{p}=\left\{p_{1}, \ldots, p_{k}\right\}$ and with negative punctures $\mathfrak{q}=\left\{q_{1}, \ldots, q_{\ell}\right\}$ on $\Sigma$. For each given Floer datum $(K, J)$ and a collection $\vec{z}=\left\{z_{*}\right\}_{* \in \mathfrak{p} \cup \mathfrak{q}}$ of asymptotic periodic orbits $z_{*}$ attached to the punctures $*=p_{i}$ or $*=q_{j}$, we consider the perturbed Cauchy-Riemann equation

$$
\left\{\begin{array}{l}
\bar{\partial}_{(K, J)}(u)=0, \\
u\left(\infty_{*}, t\right)=z_{*}(t) .
\end{array}\right.
$$


One more ingredient we need to give the definition of the Hamiltonianperturbed moduli space is the choice of an appropriate energy of the map $u$. For this purpose, we fix a metric $h_{\Sigma}$, which is compatible with the structure of the Riemann surface and which has the cylindrical ends with respect to the given cylindrical coordinates near the punctures, i.e., $h_{\Sigma}$ has the form

$$
h_{\Sigma}=d \tau^{2}+d t^{2}
$$

on $D_{*} \backslash\{*\}$. We denote by $d A_{\Sigma}$ the corresponding area element on $\Sigma$.

Here is the relevant energy function

Definition 3.2 (Energy). For a given asymptotically cylindrical pair $(K, J)$, we define

$$
E_{(K, J)}(u)=\frac{1}{2} \int_{\Sigma}\left|d u-P_{K}(u)\right|_{J}^{2} d A_{\Sigma}
$$

where $|\cdot|_{J(\sigma, u(\sigma))}$ is the norm of $\Lambda^{(0,1)}\left(u^{*} T M\right) \rightarrow \Sigma$ induced by the metrics $h_{\Sigma}$ and $g_{J}:=\omega(\cdot, J \cdot)$.

Note that this energy depends only on the conformal class of $h_{\Sigma}$, i.e., depends only on the complex structure $j$ of $\Sigma$ and restricts to the standard energy for the usual Floer trajectory moduli space given by

$$
E_{(H, J)}=\frac{1}{2} \int_{C_{*}}\left(\left|\frac{\partial u}{\partial \tau}\right|_{J}^{2}+\left|\frac{\partial u}{\partial t}-X_{H}(u)\right|_{J}^{2}\right) d t d \tau,
$$

in the cylindrical coordinates $(\tau, t)$ on the cylinder $C_{*}$ corresponding to the puncture $* . E_{(K, J)}(u)$ can be bounded by a more topological quantity depending only on the asymptotic orbits, or more precisely their liftings to the universal covering space of $\mathcal{L}_{0}(M)$, where the latter is the contractible loop space of $M$. As usual, we denote such a lifting of a periodic orbit $z$ by $[z, w]$ where $w: D^{2} \rightarrow M$ is a disc bounding the loop $z$.

We recall the definition of the standard action functional $\mathcal{A}_{H}: \widetilde{\mathcal{L}}_{0}(M) \rightarrow$ $\mathbb{R}$ on the Novikov covering space $[\mathbf{H S}]$ given by

$$
\mathcal{A}_{H}([\gamma, w])=-\int w^{*} \omega-\int_{0}^{1} H(t, \gamma(t)) d t .
$$

The following lemma can be derived by a straightforward computation. See $[\mathbf{S c 2}, \mathbf{O h} 3, \mathrm{Se} 2]$ for related calculations.

Lemma 3.1. Assume $(K, J)$ is asymptotically cylindrical. Let $\left\{\left[z_{*}, w_{*}\right]\right\}_{* \in \mathfrak{p} \cup \mathfrak{q}}$ be a given collection of asymptotic periodic orbits and let u have finite energy. Then we have the identity

$$
E_{(K, J)}(u)=\sum_{i=1}^{k} \mathcal{A}_{H_{p_{i}}}\left(\left[z_{i}^{+}, w_{i}^{+}\right]\right)-\sum_{j=1}^{\ell} \mathcal{A}_{H_{q_{j}}}\left(\left[z_{j}^{-}, w_{j}^{-}\right]\right)+\int_{\Sigma} R_{K}(u),
$$

where $R_{K} \in \Omega^{2}\left(\Sigma, C^{\infty}(M)\right)$ is the curvature two-form of the one-form $K$. 
Here, we remark that the last curvature integral converges as $R_{K}(u)$ will have compact support by the hypothesis that $K$ is cylindrical near the ends of $\dot{\Sigma}$.

We also consider the real blow-up of $\dot{\Sigma} \subset \Sigma$ at the punctures and denote it by $\bar{\Sigma}$ which is a compact Riemann surface with boundary

$$
\partial \bar{\Sigma}=\coprod_{* \in \mathfrak{p} \cup \mathfrak{q}} S_{*}^{1},
$$

where $S_{*}^{1}$ is the exceptional circle over the point $*$. We note that since there is given a preferred coordinates near the point $*$, each circle $S_{*}^{1}$ has the canonical identification

$$
\theta_{*}: S_{*}^{1} \rightarrow \mathbb{R} / \mathbb{Z}=[0,1] \bmod 1 .
$$

We note that for a given asymptotic orbits $\vec{z}$, one can define the space of maps $u: \dot{\Sigma} \rightarrow M$ which can be extended to $\bar{\Sigma}$ such that $u \circ \theta_{*}=z_{*}(t)$ for $* \in \mathfrak{p} \cup \mathfrak{q}$. Each such map defines a natural homotopy class $B$ relative to the boundary. We denote the corresponding set of homotopy classes by $\pi_{2}(\vec{z})$. When we are given the additional data of bounding discs $w_{*}$ for each $z_{*}$, then we can form a natural homology (in fact a homotopy class), denoted by $B \#\left(\coprod_{* \in \mathfrak{p} \cup \mathfrak{q}}\left[w_{*}\right]\right) \in H_{2}(M)$, by 'capping-off' the boundary components of $B$ using the discs $w_{*}$, respectively.

Definition 3.3. Let $\left\{\left[z_{*}, w_{*}\right]\right\}_{* \in \mathfrak{p} \cup \mathfrak{q}}$ be given. We say $B \in \pi(\vec{z})$ is admissible if it satisfies

$$
B \#\left(\coprod_{* \in \mathfrak{p} \cup \mathfrak{q}}\left[w_{*}\right]\right)=0 \quad \text { in } H_{2}(M, \mathbb{Z}),
$$

where

$$
\#: \pi_{2}(\vec{z}) \times \prod_{* \in \mathfrak{p} \cup \mathfrak{q}} \pi_{2}\left(z_{*}\right) \rightarrow H_{2}(M, \mathbb{Z})
$$

is the natural gluing operation of the homotopy class from $\pi_{2}(\vec{z})$ and those from $\pi_{2}\left(z_{*}\right)$ for $* \in \mathfrak{p} \cup \mathfrak{q}$. Now we are ready to give the definition of the Floer moduli spaces.

Definition 3.4. Let $(K, J)$ be a Floer datum over $\Sigma$ with punctures $\mathfrak{p}, \mathfrak{q}$, and let $\left\{\left[z_{*}, w_{*}\right]\right\}_{* \in \mathfrak{p} \cup \mathfrak{q}}$ be the given asymptotic orbits. Let $B \in \pi_{2}(\vec{z})$ be a homotopy class admissible to $\left\{\left[z_{*}, w_{*}\right]\right\}_{* \in \mathfrak{p} \cup \mathfrak{q} \text {. We define the moduli space }}$

$$
\mathcal{M}\left(K, J ;\left\{\left[z_{*}, w_{*}\right]\right\}_{*}\right)
$$

to be the set of $u: \dot{\Sigma} \rightarrow M$ that satisfies $(3.4)$ and $[u] \#\left(\coprod_{* \in \mathfrak{p} \cup \mathfrak{q}}\left[w_{*}\right]\right)=0$. 
We note that the moduli space $\mathcal{M}\left(K, J ;\left\{\left[z_{*}, w_{*}\right]\right\}_{*}\right)$ is a finite union of the moduli spaces

$$
\mathcal{M}(K, J ; \vec{z} ; B) ; \quad B \#\left(\coprod_{* \in \mathfrak{p} \cup \mathfrak{q}}\left[w_{*}\right]\right)=0: .
$$

It follows from the energy estimate (3.6) and Gromov compactness that there are only finitely many elements $B \in \pi_{2}(\vec{z})$ admissible to the given

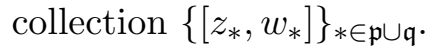

\section{Formulation of the PSS maps}

In this section, we will give a precise formulation of the so called PSS-map introduced in [PSS].

Let $f: M \rightarrow \mathbb{R}$ be a back-ground Morse function on $M$ and $H=H(t, x)$ and $J=J(t, x)$. The goal of the PSS-map is to establish an isomorphism between the Morse homology of $f$ and the Floer homology of $(H, J)$.

One of the moduli spaces entering in the construction of the PSS-map is the space of solutions of (3.4) with one puncture, which can be either positive or negative, and with one marked point playing the role of the origin of $\dot{\Sigma}$.

4.1. The smooth moduli space $\mathcal{M}_{\left(s_{0} ; s_{+}, s_{-}\right)}(\boldsymbol{K}, \boldsymbol{J} ; \boldsymbol{B})$. We consider the triple

$$
\mathfrak{p}=\left\{p_{1}, \ldots, p_{s_{+}}\right\}, \mathfrak{q}=\left\{q_{1}, \ldots, q_{s_{-}}\right\}, \mathfrak{r}=\left\{r_{1}, \ldots, r_{s_{0}}\right\}
$$

of positive and negative punctures, with analytic charts assigned, and marked points respectively. We assume they are all distinct points. We denote by

$$
\widetilde{\mathcal{M}}_{\left(s_{0} ; s_{+}, s_{-}\right)}
$$

the set of all such triples and by $\mathcal{M}_{\left(s_{0} ; s_{+}, s_{-}\right)}$the quotient space by the action of automorphisms of the punctured Riemann surface with marked points. We call a triple $(\mathfrak{r} ; \mathfrak{p}, \mathfrak{q})$ stable if it has a finite automorphism group. The space $\mathcal{M}_{\left(s_{0} ; s_{+}, s_{-}\right)}$is non-empty as long as $s_{0}+s_{+}+s_{-} \geq 3$.

Next we define $\mathcal{M}_{\left(s_{0} ; s_{+}, s_{-}\right)}(K, J ; \vec{z} ; B)$ in an obvious way,

$$
\mathcal{M}_{\left(s_{0} ; s_{+}, s_{-}\right)}(K, J ; \vec{z} ; B)=\{(u ; \mathfrak{r} ; \mathfrak{p}, \mathfrak{q}) \mid u, \text { satisfies }(3.4)[u]=B\},
$$

where $B$ is a given homotopy class of maps $u$ satisfying the asymptotic conditions at the punctures. Here $\vec{z}=\left\{z_{*}\right\}_{* \in \mathfrak{p} \cup \mathfrak{q}}$ is a given set of asymptotic periodic orbits. To avoid having continuous automorphisms, we will always assume that the asymptotic Hamiltonian $H$ at the puncture is not timeindependent when we consider the moduli space corresponding to

$$
\left(s_{0} ; s_{+}, s_{-}\right)=(1 ; 1,0) \text { or }(1 ; 0,1) .
$$


We will not need to consider the case where $r=0, s_{+}+s_{-}=1$. This assumption rules out the possibility of a circle symmetry for the asymptotic solutions at infinity.

In addition, we will also assume that $K$ and $J$ satisfy

$$
\begin{aligned}
K & \equiv 0, \\
J & \equiv J_{0}, \quad \text { near the marked point } r \in \dot{\Sigma},
\end{aligned}
$$

respectively, where $J_{0}$ is a (time-independent) compatible almost complex structure of $(M, \omega)$. We assume $J_{0}$ is generic.

This assumption together with the condition on the asymptotic Hamiltonian being nondegenerate makes such $K$ prevent from having continuous symmetry and so a genuinely two-dimensional family over $\dot{\Sigma}$. In particular, any solution in these moduli space has automatically a finite automorphism group which can be trivial. The following can be derived by a standard argument.

Proposition 4.1. Let $J_{0}$ be a given compatible almost complex structure on $(M, \omega)$. Suppose that all the asymptotic pairs $\left(H_{*}, J_{*}\right)$ are Floer-regular in that $H_{*}$ are non-degenerate in the sense of Lefshetz fixed point theory, and in that the corresponding Floer moduli space is transverse. Then there exists a generic choice of such $(K, J) \in \mathcal{K}_{\dot{\Sigma}} \times \mathcal{J}_{\dot{\Sigma}}$ such that the moduli space $\mathcal{M}_{\left(s_{0} ; s_{+}, s_{-}\right)}((K, J) ; \vec{z} ; B)$ become transverse. Furthermore, the dimension of the moduli space is given by

$$
\begin{aligned}
\operatorname{dim} \mathcal{M}_{\left(s_{0} ; s_{+}, s_{-}\right)}(K, J ; \vec{z} ; B)= & \sum \mu_{H_{*}^{+}}\left(\left[z_{*}^{+}, w_{*}^{+}\right]\right)-\sum \mu_{H_{*}^{-}}\left(\left[z_{*}^{-}, w_{*}^{-}\right]\right) \\
& +2 s_{0}+n\left(s_{+}-s_{-}\right),
\end{aligned}
$$

where $\left[z_{*}, w_{*}\right]$ are the liftings of the asymptotic orbits with $B$ satisfying (3.7).

From now on in the rest of the paper, we will exclusively concern $\Sigma$ of genus zero.

4.2. The PSS maps $\boldsymbol{\Phi}$ and $\boldsymbol{\Psi}$. In this subsection, we recall the definitions of the two PSS maps $\Phi$ and $\Psi$ from [PSS] except that we follow different grading conventions using the ones from $[\mathbf{O h 2}]$ for the various grading issues (Figure 1). And we also use Morse cycles of $-f$, instead of $f$, to represent the homology of $M$ : In particular, the grading of Morse cycles is given by

$$
\operatorname{Index}_{(-f)}(p)=2 n-\operatorname{Index}_{f}(p) .
$$

The issue of grading is not essential for the proof and so can be largely ignored. We just put this here for the consistency with the papers by the senior author $[\mathbf{O h 2}]-[$ Oh4 $]$.

Let $\dot{\Sigma}_{+}$be the Riemann sphere with one marked point $o_{+}$and one positive puncture $e_{+}$. We identify $\dot{\Sigma}_{+} \backslash\left\{o_{+}\right\} \cong \mathbb{R} \times S^{1}$ and denote by $(\tau, t)$ the 


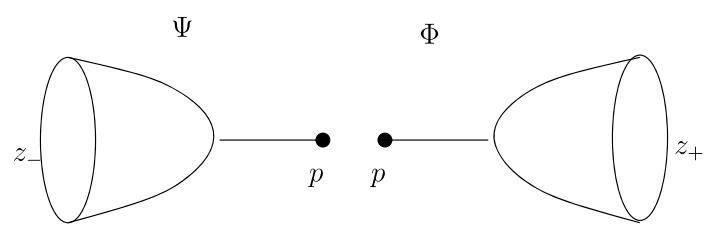

Figure 1. The PSS maps $\Psi$ and $\Phi$.

corresponding coordinates so that $\{+\infty\} \times S^{1}$ correspond to $e_{+}$. We note that the coordinates $(\tau, t)$ is defined modulo the the action of $\mathbb{R} \times S^{1} \cong \mathbb{C}^{*}$

$$
(\tau, t) \mapsto(\tau+a, t+b) .
$$

We consider the one form $K_{+} \in \Omega^{1}(\dot{\Sigma}, \operatorname{ham}(M, \omega))$ such that in the above mentioned coordinates $K_{+}$as a one-form in $\Omega^{1}(\dot{\Sigma}, \operatorname{ham}(M, \omega))$ can be written as

$$
\begin{cases}K_{+}=0, & \text { near } o_{+}, \\ K_{+}=H_{+}(t, x) d t, & \text { near } e_{+},\end{cases}
$$

where $H_{+}: S^{1} \times M \rightarrow \mathbb{R}$ is a $t$-dependent Hamiltonian function. By the remark on the coordinate $(\tau, t)$ made above, the phrases "near $e_{+}$" or "near $o_{+}$" put on the above definition of $K_{+}$does not depend on the choice of coordinates and has well-defined meaning.

Let $\mathcal{L}_{0}(M)$ be the contractible free loop space of $M$ and $\mathcal{L}_{0}(M)$ the Novikov covering space $\widetilde{\mathcal{L}}_{0}(M)$. Let $z_{+}=z_{+}(t)\left(t \in S^{1}\right)$ be a nondegenerate periodic orbit of $H_{+}(t, x)$ We denote by $\left[z_{+}, w_{+}\right]$a lifting of $z_{+}$to $\widetilde{\mathcal{L}}_{0}(M)$ and denote

$$
\widetilde{\operatorname{Per}}\left(H_{+}\right)=\left\{\left[z_{+}, w_{+}\right] \mid \dot{z}_{+}=X_{H_{+}}\left(z_{+}\right)\right\}
$$

We note that $\widetilde{\operatorname{Per}}\left(H_{+}\right)$is precisely the set of critical points of the action functional $\mathcal{A}_{H}: \widetilde{\mathcal{L}}_{0}(M) \rightarrow \mathbb{R}$. Using the bounding disc $w_{+}: D^{2} \rightarrow M$, we trivialize the symplectic bundle $z_{+}^{*}(T M)$ and get a loop in $S p(2 n)$, which gives rise to the Conley-Zehnder index $\mu_{H_{+}}\left(\left[z_{+}, w_{+}\right]\right) \in \mathbb{Z}$.

Now we consider the moduli space

$$
\begin{aligned}
\mathcal{M}\left(K_{+}, J_{+} ;\left[z_{+}, w_{+}\right] ; A_{+}\right)= & \left\{u: \dot{\Sigma} \rightarrow M \mid \bar{\partial}_{\left(K_{+}, J_{+}\right)} u=0,\right. \\
& \left.u(+\infty, t)=z_{+}(t),\left[u \# w_{+}\right]=A_{+}\right\},
\end{aligned}
$$

where $A_{+} \in H_{2}(M, \mathbb{Z})$ is in the image of the Hurwitz map $\pi_{2}(M) \rightarrow H_{2}$ $(M, \mathbb{Z})$.

For generic $J_{+}$or $K_{+}$, the moduli space is regular and its dimension is equal to

$$
\text { Index } D_{u} \bar{\partial}_{\left(K_{+}, J_{+}\right)}=n-\mu_{H_{+}}\left(\left[z_{+}, w_{+}\right]\right)+2 c_{1}\left(A_{+}\right) \text {. }
$$


Here we follow the conventions from [Oh2] (See section $6.2[\mathbf{O h 2}]$ ). Similarly for $u$ in the moduli space

$$
\begin{aligned}
\mathcal{M}\left(K_{-}, J_{-} ;\left[z_{-}, w_{-}\right] ; A_{-}\right)= & \left\{u: \dot{\Sigma} \rightarrow M \mid \bar{\partial}_{\left(K_{-}, J_{-}\right)} u=0,\right. \\
& \left.u(-\infty, t)=z_{-}(t),\left[\bar{w}_{-} \# u\right]=A_{-}\right\},
\end{aligned}
$$

where $A_{-}$is similar to $A_{+}$.

$$
\text { Index } D_{u} \bar{\partial}_{\left(K_{-}, J_{-}\right)}=n+\mu_{H_{-}}\left(\left[z_{-}, w_{-}\right]\right)+2 c_{1}\left(A_{-}\right) \text {. }
$$

Recall that the quantum homology $Q H_{*}(M)=H_{*}(M) \otimes \Lambda_{\omega}$, where $\Lambda_{\omega}$ is the Novikov ring defined as

$$
\begin{aligned}
\Lambda_{\omega}= & \left\{\sum_{A \in \Gamma} r_{A} q^{-A} \mid r_{A} \in \mathbb{Q}, \text { such that for all } \lambda \in \mathbb{R},\right. \\
& \left.\#\left\{A \in \Gamma \mid r_{A} \neq 0, \omega(A)>\lambda\right\}<\infty\right\} .
\end{aligned}
$$

Here $\Gamma \subset H_{2}(M)$ is the image of $\pi_{2}(M)$ under the Hurewicz homomorphism, and $q$ is a formal variable. If we use the Morse homology of $-f$ to represent $H_{*}(M)$, then we can represent $Q H_{*}(M)$ as the homology of $C_{*}(-f) \otimes \Lambda_{\omega}$, where $C_{*}(-f)$ is the chain complex of the Morse homology of $-f$ generated by the critical points of $f$. The grading of $[p] q^{-A}$ is $\mu_{(-f)}(p)-2 c_{1}(A)$, where $[p] \in C_{*}(-f)$, and $\mu_{(-f)}(p)$ is the Morse index of $f$ at $p$.

We are going to define the PSS map

$$
\Phi_{*}: Q H_{k}(M) \rightarrow F H_{n-k}(M) .
$$

Following [PSS], we first define the chain level map $\Phi: C_{*}(-f) \otimes \Lambda_{\omega} \rightarrow$ $C F_{*}(M)$ by defining it on the generators $[p]$ of $C_{*}(-f)$ and then linearly extending over ring $\Lambda_{\omega}$ as

$$
\Phi:[p] \rightarrow \sum_{\left[z_{+}, w_{+}\right] \in \widetilde{\operatorname{Per}}\left(H_{+}\right)} \#\left(\mathcal{M}\left(p,\left[z_{+}, w_{+}\right] ; A_{+}\right)\left[z_{+}, w_{+}\right] q^{-A_{+}}\right.
$$

Here, roughly speaking, the moduli space $\mathcal{M}\left(p,\left[z_{+}, w_{+}\right] ; A_{+}\right)$consists of "spike discs" emerging from the critical point $p$ and ending on the periodic orbit $z_{+}$in class $\left[u \# w_{+}\right]=A_{+}$in $\Gamma$. More precisely, we have the definition

$$
\begin{aligned}
\mathcal{M}\left(p,\left[z_{+}, w_{+}\right] ; A_{+}\right)= & \left\{\left(\chi_{+}, u_{+}\right) \mid u_{+}: \dot{\Sigma}_{+} \rightarrow M,\left[u_{+} \# w_{+}\right]=A_{+},\right. \\
& u(+\infty, t)=z_{+}(t), \bar{\partial}_{\left(K_{+}, J_{+}\right)} u_{+}=0, \\
& \left.\dot{\chi}_{+}=\nabla f\left(\chi_{+}\right), \chi_{+}(-\infty)=p, \chi_{+}(0)=u_{+}\left(o_{+}\right)\right\} .
\end{aligned}
$$

Here we put index condition such that $\mathcal{M}\left(p,\left[z_{+}, w_{+}\right] ; A_{+}\right)$is a zerodimensional oriented manifold so we can do algebraic count "\#". The index 
condition is

$$
\begin{aligned}
& \left(n-\mu\left(\left[z_{+}, w_{+}\right]+2 c_{1}\left(A_{+}\right)\right)+(2 n-\mu(p))-2 n=0,\right. \\
& \text { i.e. } \quad \mu\left(\left[z_{+}, w_{+}\right]\right)=n-\left(\mu(p)-2 c_{1}\left(A_{+}\right)\right) .
\end{aligned}
$$

Standard gluing argument shows that $\phi$ is a chain map (similar to the continuation map that proves Morse homology is independent on the Morse function), so it passes to homology and we get the PSS map $\Phi_{*}: Q H_{k}(M) \rightarrow$ $H F_{n-k}(M)$.

Next we define the inverse of $\Phi$

$$
\Psi_{*}: H F_{*}(M) \rightarrow Q H_{*}(M) .
$$

For any $\left[z_{-}, w_{-}\right] \in \widetilde{\operatorname{Per}}\left(K_{-}\right)$, define $\psi: C F_{*}(M) \rightarrow C_{*}(-f) \otimes \Lambda_{\omega}$,

$$
\Psi:\left[z_{-}, w_{-}\right] \rightarrow \sum_{p \in \operatorname{Crit}(-f) ; A_{-} \in \pi_{2}(M)} \# \mathcal{M}\left(\left[z_{-}, w_{-}\right], p ; A_{-}\right) p \otimes q^{-A_{-}},
$$

where $\mathcal{M}\left(\left[z_{-}, w_{-}\right], p ; A_{-}\right)$consists of "spiked-discs" emerging from the periodic orbit $z_{-}$and ending on the critical point $p$, namely

$$
\begin{aligned}
\mathcal{M}\left(\left[z_{-}, w_{-}\right], p ; A_{-}\right)= & \left\{\left(u_{-}, \chi_{-}\right) \mid u_{-}: \dot{\Sigma}_{-} \rightarrow M,\left[\bar{w}_{-} \# u_{-}\right]=A_{-},\right. \\
& u(-\infty, t)=z_{-}(t), \bar{\partial}_{\left(K_{-}, J_{-}\right)} u_{-}=0, \\
& \left.\dot{\chi}_{-}=\nabla f\left(\chi_{-}\right), \chi_{-}(+\infty)=p, \chi_{-}(0)=u_{-}\left(o_{-}\right)\right\} .
\end{aligned}
$$

Here we also put the index condition

$$
\mu_{H_{-}}\left(\left[z_{-}, w_{-}\right]\right)=n-\left(\mu_{(-f)}(p)-2 c_{1}\left(A_{-}\right)\right),
$$

so $\mathcal{M}\left(\left[z_{-}, w_{-}\right], p ; A_{-}\right)$becomes a zero-dimensional (orientable) manifold. The same continuation map argument shows $\Psi$ is a chain map so it induce the homomorphism $\Psi_{*}: H F_{n-k}(M) \rightarrow Q H_{k}(M)$.

4.3. The PSS-scheme of proof of the isomorphism property $\Phi_{*}$. In this section, we sketch the argument of Piunikhin-Salamon-Schwarz towards a proof of isomorphism property of the PSS-maps based on some picture, which describes a deformation leading to the chain isomorphism between the composition

$$
\Psi \circ \Phi, \quad \mathrm{i} d: C F^{*}(M) \rightarrow C F^{*}(M)
$$

and the identity map. The deformation involves the moduli spaces of three different types in the course of deformations (see Figure 2):

(1) disk-flow-disk,

(2) nodal Floer trajectories,

(3) chain map Floer trajectories.

For the sake of following discussion, we denote the deformation parameter by $\lambda \in[-1,1]$, so that the nodal configuration occurs at $\lambda=0$. As long as $\lambda>0$ or $\lambda<0$, the deformation involves the same type of moduli 

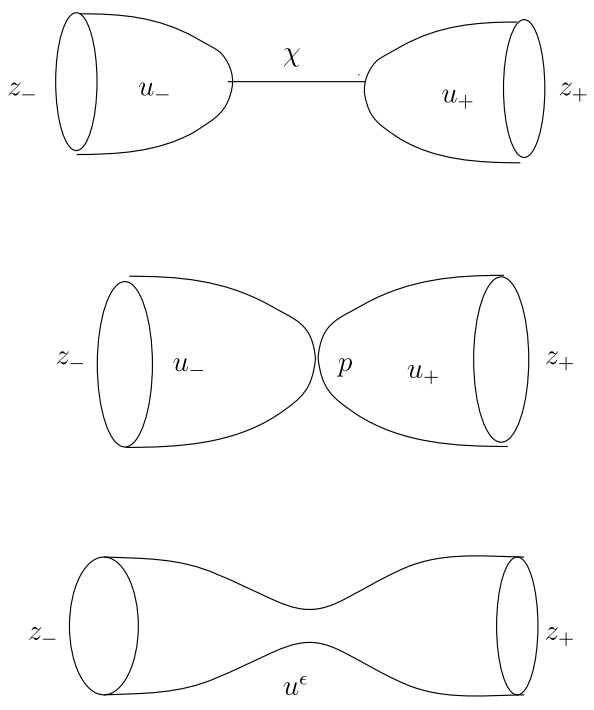

Figure 2. The PSS scheme.

spaces and so can be applied the standard argument to construct a corbodism over $\left[-1,-\varepsilon_{0}\right]$ or $\left[\varepsilon_{0}, 1\right]$ for $\varepsilon>0$. To complete the cobordism over the whole interval $[-1,1]$, one needs to connect the two cobordisms to one over $\left[-\varepsilon_{0}, \varepsilon_{0}\right]$. However, there occurs "phase change" in the moduli spaces over the interval $\left[-\varepsilon_{0}, \varepsilon_{0}\right]$ at $\lambda=0$. Due to the "phase change" at $\lambda=0$, one can a priori expect only a piecewise smooth corbodism and needs to prove a bi-collar theorem of $\mathcal{M}_{0} \subset \mathcal{M}^{\text {para }}$ to materialize the PSS-scheme. From $-\varepsilon_{0}$ to 0 , one can construct the left one-sided collar by finite dimensional differential topology (See Section 9). For the right one-sided collar over $\left[0, \varepsilon_{0}\right]$, we will construct the collar by the method of adiabatic degeneration $[$ Oh5, Oh3, MT] and scale-dependent gluing of immersed nodal Floer trajectories developed in the present paper

This then implies the following isomorphism property as stated in [PSS]. We refer to Part III in the present paper for the details of proof of this isomorphism property based on this bi-collar neighborhood theorem and the adiabatic degeneration result. This final step largely reproduces the argument used in [PSS].

Theorem 4.1. Let $(f ; g)$ be a generic Morse-Smale pair of a Morse function $f$ and a metric $g$ on $M$ and $H^{M o r s e}(f ; g)$ the Morse homology of $(f ; g)$ and $(H, J)$ be a generic time-periodic Hamiltonian function $H$ and a family of compatible almost complex structure $J=\left\{J_{t}\right\}$ on $M$. Let $\Psi, \Phi$ be the PSS maps given in $[\boldsymbol{P S S}]$. Then there exists a homomorphism

$$
h_{p s s}: C F^{*}(H, J) \rightarrow C F^{*+1}(H, J)
$$


that satisfy

$$
\Psi \circ \Phi-i d=\partial_{(H, J)} \circ h_{p s s}-h_{p s s} \circ \partial_{(f, g)} .
$$

In particular, we have $\Psi_{*} \circ \Phi_{*}=i d$ in homology.

This shows $\Psi_{*} \circ \Phi_{*}=i d$. The other identity $\Phi_{*} \circ \Psi_{*}=i d$ is much easier to prove. Details of the proof are given in Section 15.

Remark 4.1. The adiabatic degeneration of the moduli space of solutions of the Floer trajectory equation with a Morse function $\varepsilon f$ in the middle does not produces just nodal Floer trajectories as used in the PSS-scheme but produces the nodal Floer trajectories with some one-jet datum which reflects the back ground Morse function $f$. This datum enters in our scaledependent gluing which is the correct reversal process of the the adiabatic degeneration of the moduli space as $\varepsilon \rightarrow 0$.

\section{Part I. Geometry: Floer trajectories with immersed nodes}

\section{Definition of the deformation-cobordism moduli space}

In this section, we will provide the precise mathematical formulation of the moduli spaces appearing in each stage of the deformation-cobordism described in Subsection 4.3 which was proposed by Piunikhin-SalamonSchwarz [PSS, MS].

5.1. Moduli space of "disk-flow-disk" configurations. This subsection is the first stage of the deformation of the parameterized moduli space entering in the construction of the chain homotopy map between $\Psi \circ \Phi$ and the identity on $H F(H, J)$.

A "disk-flow-disk" configuration consists of two perturbed $\boldsymbol{J}$-holomorphic discs joined by a gradient flow line between their marked points. In this section we will define the moduli space of such configurations.

For notation brevity, we just denote

$$
\mathcal{M}^{\varepsilon}\left(K^{ \pm}, J^{ \pm} ;\left[z_{ \pm}, w_{ \pm}\right], f ; A_{ \pm}\right)=\mathcal{M}^{\varepsilon}\left(\left[z_{ \pm}, w_{ \pm}\right], f ; A_{ \pm}\right),
$$

respectively, omitting the Floer datum $\left(K^{ \pm}, J^{ \pm}\right)$, as long as it does not cause confusion.

Given the two moduli spaces $\mathcal{M}\left(\left[z_{-}, w_{-}\right] ; A_{-}\right)$and $\mathcal{M}\left(\left[z_{+}, w_{+}\right] ; A_{+}\right)$and the Morse function $f$, let the moduli space of "disk-flow-disk" configurations $\left(u_{-}, \chi, u_{+}\right)$of flow time $\varepsilon$ to be

$$
\begin{gathered}
\mathcal{M}^{\varepsilon}\left(\left[z_{-}, w_{-}\right] ; f ;\left[z_{+}, w_{+}\right] ; A_{ \pm}\right):=\left\{\left(u_{-}, \chi, u_{+}\right) \mid u_{ \pm} \in \mathcal{M}\left(K^{ \pm}, J^{ \pm} ; \vec{z}_{ \pm} ; A_{ \pm}\right)\right. \\
\left.\chi:[0, \varepsilon] \rightarrow M, \dot{\chi}-\nabla f(\chi)=0, u_{-}\left(o_{-}\right)=\chi(0), u_{+}\left(o_{+}\right)=\chi(\varepsilon)\right\} .
\end{gathered}
$$


Then the moduli space of "disk-flow-disk" configurations is defined to be

$$
\mathcal{M}^{\text {para }}\left(\left[z_{-}, w_{-}\right] ; f ;\left[z_{+}, w_{+}\right] ; A_{ \pm}\right):=\bigcup_{\varepsilon \geq 0} \mathcal{M}^{\varepsilon}\left(\left[z_{-}, w_{-}\right] ; f ;\left[z_{+}, w_{+}\right] ; A_{ \pm}\right)
$$

Note we have included the $\varepsilon=0$ case, which corresponds to the nodal Floer trajectory moduli space.

We now provide the off-shell formulation of the "disk-flow-disk" moduli spaces. We first provide the Banach manifold hosting the moduli space

$$
\mathcal{M}^{\varepsilon}\left(\left[z_{-}, w_{-}\right] ; f ;\left[z_{+}, w_{+}\right] ; A_{ \pm}\right) .
$$

We define

$$
\begin{aligned}
\mathcal{B}_{\varepsilon}^{\mathrm{dfd}}\left(z_{-}, z_{+}\right):= & \left\{\left(u_{-}, \chi, u_{+}\right) \mid u_{ \pm} \in W^{1, p}\left(\dot{\Sigma}, M ; z_{ \pm}\right),\right. \\
& \left.\chi \in W^{1, p}([0, \varepsilon], M), u_{-}\left(o_{-}\right)=\chi(0), u_{+}\left(o_{+}\right)=\chi(\varepsilon)\right\}
\end{aligned}
$$

for $p>2$. Then for each $u=\left(u_{-}, \chi, u_{+}\right) \in \mathcal{B}_{\varepsilon}^{\text {res }}\left(z_{-}, z_{+}\right)$, we define

$$
L_{u}^{p}\left(z_{-}, z_{+}\right)=L^{p}\left(\Lambda^{(0,1)} u^{*} T M\right)
$$

and form the Banach bundle

$$
\mathcal{L}_{\varepsilon}^{\mathrm{dfd}}=\bigcup_{u \in \mathcal{B}_{\varepsilon}^{\mathrm{ddd}}\left(z_{-}, z_{+}\right)} L_{u}^{p}\left(z_{-}, z_{+}\right)
$$

over $\mathcal{B}_{\varepsilon}^{\text {dfd }}\left(z_{-}, z_{+}\right)$. Here the superscript "dfd" stands for "disk-flow-disk". We refer to $[\mathbf{F l 2}]$ for a more detailed description of the asymptotic behavior of the elements in $\mathcal{B}_{\varepsilon}^{\mathrm{dfd}}\left(z_{-}, z_{+}\right)$in the context of Floer moduli spaces.

For $u=\left(u_{-}, \chi, u_{+}\right) \in \mathcal{B}_{\varepsilon}^{\mathrm{dfd}}\left(z_{-}, z_{+}\right)$, its tangent space $T_{u} \mathcal{B}_{\varepsilon}^{\mathrm{dfd}}$ consists of $\xi=\left(\xi_{-}, a, \xi_{+}\right)$, where $\xi_{ \pm} \in W^{1, p}\left(u_{ \pm}^{*} T M\right), a \in W^{1, p}\left(\chi^{*} T M\right)$, with the matching condition

$$
\xi_{-}\left(o_{-}\right)=a(0), \quad \xi_{+}\left(o_{+}\right)=a(\varepsilon) .
$$

We denote the set of such $\xi$ as $W_{u}^{1, p}\left(z_{-}, z_{+}\right)$.

We let

$$
\mathcal{B}^{\mathrm{dfd}}\left(z_{-}, z_{+}\right)=\bigcup_{\varepsilon \in\left(0, \varepsilon_{0}\right)} \mathcal{B}_{\varepsilon}^{\mathrm{dfd}}\left(z_{-}, z_{+}\right) \quad \text { and } \quad \mathcal{L}^{\mathrm{dfd}}\left(z_{-}, z_{+}\right)=\bigcup_{\varepsilon \in\left(0, \varepsilon_{0}\right)} \mathcal{L}_{\varepsilon}^{\mathrm{dfd}}\left(z_{-}, z_{+}\right) .
$$

Remark 5.1. If we regard $u$ in $\mathcal{B}^{\text {dfd }}$ instead of $\mathcal{B}_{\varepsilon}^{\text {dfd }}$, then its tangent space consists of $\xi=\left(\xi_{-}, a, \xi_{+}, \mu\right)$, where $\xi_{ \pm} \in W^{1, p}\left(u_{ \pm}^{*} T M\right), a \in W^{1, p}$ $\left(\chi^{*} T M\right), \mu \in T_{\varepsilon} \mathbb{R} \cong \mathbb{R}$, with the matching condition

$$
\xi_{-}\left(o_{-}\right)=a(0), \quad \xi_{+}\left(o_{+}\right)=a(\varepsilon)+\mu \nabla f(\chi(\varepsilon)) .
$$

Here the $\mu$ comes from the variation of the length $\varepsilon$ of the domain of gradient flows. 
Now we fix $\varepsilon>0$ and consider a natural section

$$
e: \mathcal{B}_{\varepsilon}^{\mathrm{dfd}}\left(z_{-}, z_{+}\right) \rightarrow \mathcal{L}_{\varepsilon}^{\mathrm{dfd}}\left(z_{-}, z_{+}\right)
$$

such that $e(u) \in L_{u}^{p}\left(z_{-}, z_{+}\right)$is given by

$$
e(u)=\left(\bar{\partial}_{\left(K_{-}, J_{-}\right)} u_{-}, \dot{\chi}-\nabla f(\chi), \bar{\partial}_{\left(K_{+}, J_{+}\right)} u_{+}\right),
$$

where the $u_{ \pm}$and $\chi$ satisfy the matching condition in (5.3). The linearization of $e$ at $u \in \mathrm{e}^{-1}(0)=\mathcal{M}^{\varepsilon}\left(\left[z_{-}, w_{-}\right] ; f ;\left[z_{+}, w_{+}\right] ; A_{ \pm}\right)$induces a linear operator

$$
E(u):=D_{u} e: T_{u} \mathcal{B}_{\varepsilon}^{\mathrm{dfd}}\left(z_{-}, z_{+}\right) \rightarrow L_{u}^{p}\left(z_{-}, z_{+}\right),
$$

where we have

$$
\begin{aligned}
T_{u} \mathcal{B}_{\varepsilon}^{\mathrm{dfd}}\left(z_{-}, z_{+}\right) & =\left\{\left(\xi_{-}, \xi_{+}, a\right) \mid \xi_{ \pm} \in W^{1, p}\left(u_{ \pm}^{*} T M\right), \xi_{-}\left(o_{-}\right)\right. \\
& \left.=a(0), \xi_{+}\left(o_{+}\right)=a(\varepsilon)\right\}
\end{aligned}
$$

and the value $D_{u} e(\xi)=: \eta$ has the expression

$$
\eta=\left(\eta_{-}, b, \eta_{+}\right)=\left(D_{u_{-}} \bar{\partial}_{\left(K_{-}, J_{-}\right)}\left(\xi_{-}\right), \frac{D a}{d \tau}-\nabla_{a} \operatorname{grad}(f), D_{u_{+}} \bar{\partial}_{\left(K_{+}, J_{+}\right)}\left(\xi_{+}\right)\right)
$$

for $\xi=\left(\xi_{-}, a, \xi_{+}\right)$. For the simplicity of notation, we denote the tangent space

$$
T_{u} \mathcal{B}_{\varepsilon}^{\mathrm{dfd}}\left(z_{-}, z_{+}\right) \subset W^{1, p}\left(u_{-}^{*} T M\right) \times W^{1, p}\left(\chi^{*} T M\right) \times W^{1, p}\left(u_{+}^{*} T M\right)
$$

by $W_{u}^{1, p}\left(z_{-}, z_{+} ; \mathrm{dfd}\right)$. Now we show $E(u)$ is Fredholm and compute its index. Denote by $\Pi_{0}^{\varepsilon}$ the parallel transport along the path $\chi$ and $\Delta_{u(o)}$ the diagonal subspace in $T_{u(o)} M \times T_{u(o)} M$.

Proposition 5.1. The operator E $(u)$ is a Fredholm operator and we have

$$
\operatorname{Index} E(u)=\mu_{H_{-}}\left(\left[z_{-}, w_{-}\right]\right)-\mu_{H_{+}}\left(\left[z_{+}, w_{+}\right]\right)+2 c_{1}\left(A_{-}\right)+2 c_{1}\left(A_{+}\right) .
$$

for any

$$
u=\left(u_{-}, \chi, u_{+}\right) \in \mathcal{M}^{\varepsilon}\left(\left[z_{-}, w_{-}\right] ; f ;\left[z_{+}, w_{+}\right] ; A_{ \pm}\right) .
$$

Proof. We compute the kernel and the cokernel of

$$
E(u): W_{u}^{1, p}\left(z_{-}, z_{+} ; \mathrm{dfd}\right) \rightarrow L_{u}^{p}\left(z_{-}, z_{+}\right) .
$$

By the matching condition (5.5) it is clear that

$$
\begin{aligned}
\operatorname{ker} E(u)= & \left\{\left(\xi_{-}, \xi_{+}, a\right) \mid \xi_{ \pm} \in \operatorname{ker} D_{u_{ \pm}} \bar{\partial}_{\left(K^{ \pm}, J^{ \pm}\right)},\right. \\
& \left.\frac{D a}{\partial \tau}-\nabla_{a} \operatorname{grad} f(\chi)=0, \xi_{-}\left(o_{-}\right)=a(0), \xi_{+}\left(o_{+}\right)=a(\varepsilon)\right\}
\end{aligned}
$$

It is easy to see

$$
\left(\xi_{-}\left(o_{-}\right), \xi_{+}\left(o_{+}\right)\right)=\left(d \phi_{f}^{\varepsilon} \times \mathrm{id}\right)^{-1}\left(\Delta_{u_{+}\left(o_{+}\right)}\right),
$$


for any $\left(\xi_{-}, \xi_{+}, a\right) \in \operatorname{ker} E(u)$ noticing that $a$ is determined by its initial value $a(\varepsilon)$ and by the equation

$$
\frac{D a}{\partial \tau}-\nabla_{a} \operatorname{grad} f(\chi)=0
$$

Then we derive

$$
\begin{aligned}
\operatorname{dim} \operatorname{ker} E(u)= & \operatorname{dim} \operatorname{ker} D_{u_{-}} \bar{\partial}_{\left(K^{-}, J^{-}\right)}+\operatorname{dim} \operatorname{ker} D_{u_{+}} \bar{\partial}_{\left(K_{+}, J_{+}\right)} \\
& -2 n+\operatorname{dim} \Delta_{u_{-}\left(o_{-}\right)} \cap \operatorname{Graph}\left(\left(\Pi_{0}^{\varepsilon}\right)^{-1} \circ d \phi_{f}^{\varepsilon}\right) .
\end{aligned}
$$

Next we compute the cokernel of $E(u)$. Let $E(u)^{*}$ be the adjoint operator of $E(u)$, such that

$$
E(u)^{*}: L_{u}^{p}\left(z_{-}, z_{+}\right)^{*} \rightarrow W^{1, p}\left(z_{-}, z_{+} ; \mathrm{dfd}\right)^{*} .
$$

Using the nondegenerate $L^{2}$ pairing

$$
L^{p}\left(\Lambda^{(0,1)} u^{*} T M\right) \times L^{q}\left(\Lambda^{(1,0)} u^{*} T M\right) \rightarrow \mathbb{R},
$$

we identify $L_{u}^{p}\left(z_{-}, z_{+}\right)^{*}$ with $L^{q}\left(\Lambda^{(1,0)} u^{*} T M\right)$. On the other hand, we can identify $W^{1, p}\left(z_{-}, z_{+} ; \mathrm{dfd}\right)^{*}$ with the space

$$
\left\{\left(\xi_{-}, a, \xi_{+}\right) \in W^{1, p}\left(z_{-}, z_{+}\right) \mid \xi_{-}\left(o_{-}\right)=a(0), \xi_{+}\left(o_{+}\right)=a(\varepsilon)\right\}^{\perp}
$$

in the direct product

$$
W^{-1, q}\left(z_{-}, z_{+}\right)=W^{-1, q}\left(u_{-}^{*} T M\right) \times W^{-1, q}\left(\chi^{*} T M\right) \times W^{-1, q}\left(u_{+}^{*} T M\right),
$$

where $(\cdot)^{\perp}$ denotes the $L^{2}$-orthogonal complement. Here we have $1<q<2$ since $2<p<\infty$.

We denote by

$$
E(u)^{\dagger}: L^{q}\left(\Lambda^{(1,0)} u^{*} T M\right) \rightarrow W^{-1, q}\left(z_{-}, z_{+} ; \mathrm{dfd}\right)
$$

the corresponding $L^{2}$-adjoint with respect to these identifications.

Now we derive the formula for $E(u)^{\dagger}$. Recall by definition, we have

$$
\langle E(u) \xi, \eta\rangle=\left\langle\xi, E(u)^{\dagger} \eta\right\rangle .
$$

Then for any given $\eta:=\left(\eta_{-}, b, \eta_{+}\right) \in \operatorname{ker} E^{\dagger}(u) \subset W^{-1, q}\left(z_{-}, z_{+}\right)$it satisfies

$$
\begin{aligned}
0= & \int_{0}^{\varepsilon}\left\langle\frac{D a}{\partial \tau}-\nabla \operatorname{grad} f(\chi) a, b\right\rangle \\
& +\int_{\dot{\Sigma}_{-}}\left\langle D_{u_{-}} \bar{\partial}_{\left(K^{-}, J^{-}\right)} \xi_{-}, \eta_{-}\right\rangle+\int_{\dot{\Sigma}_{+}}\left\langle D_{u_{+}} \bar{\partial}_{\left(K_{+}, J_{+}\right)} \xi_{+}, \eta_{+}\right\rangle,
\end{aligned}
$$

for all $\left(\xi_{+}, a, \xi_{-}\right) \in W_{u}^{1, p}\left(z_{-}, z_{+} ; \mathrm{dfd}\right)$, i.e., for all the triples satisfying the matching condition

$$
\xi_{-}\left(o_{-}\right)=a(0), \quad \xi_{+}\left(o_{+}\right)=a(\varepsilon) .
$$


Integrating by parts, we have

$$
\begin{aligned}
0= & \langle a(\varepsilon), b(\varepsilon)\rangle-\langle a(0), b(0)\rangle+\int_{0}^{\varepsilon}\left\langle-\frac{D b}{\partial \tau}-\nabla \operatorname{grad} f(\chi) b, a\right\rangle \\
& -\int_{\dot{\Sigma}_{-}}\left\langle\left(D_{u_{-}} \bar{\partial}_{\left(K^{-}, J^{-}\right)}\right)^{\dagger} \eta_{-}, \xi_{-}\right\rangle-\int_{\dot{\Sigma}_{+}}\left\langle\left(D_{u_{+}} \bar{\partial}_{\left(K^{+}, J^{+}\right)}\right)^{\dagger} \eta_{+}, \xi\right\rangle .
\end{aligned}
$$

Here $\left.D_{u_{ \pm}} \partial_{\left(K^{ \pm}, J^{ \pm}\right)}\right)$is the formal adjoint of $\left.D_{u_{ \pm}} \bar{\partial}_{\left(K^{ \pm}, J^{ \pm}\right)}\right)$which has its symbol of that of the Dolbeault operator $\partial$ (near $z=0$ in $\mathbb{C}$ ) and so elliptic. Here we note that we are using a metric on $\Sigma_{ \pm} \cong \mathbb{C}$ that is standard near the origin and cylindrical near the end.

Substituting (5.12) into this we can rewrite (5.13) into

$$
\begin{aligned}
0= & \int_{0}^{\varepsilon}\left\langle-\frac{D b}{\partial \tau}-\nabla \operatorname{grad} f(\chi) b, a\right\rangle+\left\langle\xi_{+}\left(o_{+}\right), b(\varepsilon)\right\rangle-\left\langle\xi_{-}\left(o_{-}\right), b(0)\right\rangle \\
& -\int_{\dot{\Sigma}_{-}}\left\langle\left(D_{u_{-}} \bar{\partial}_{\left(K^{-}, J^{-}\right)}\right)^{\dagger} \eta_{-}, \xi_{-}\right\rangle-\int_{\dot{\Sigma}_{+}}\left\langle\left(D_{u_{+}} \bar{\partial}_{\left(K^{+}, J^{+}\right)}\right)^{\dagger} \eta_{+}, \xi\right\rangle .
\end{aligned}
$$

Note $a$ can be varied arbitrarily on the interior $(0, \varepsilon)$ and can be matched to any given $\xi_{ \pm}\left(o_{ \pm}\right)$at $0, \varepsilon$. Therefore, considering the variation $\xi_{-}=\xi_{+}=0$, we derive that $b$ must satisfy

$$
\left\langle-\frac{D b}{\partial \tau}-\nabla \operatorname{grad} f(\chi) b, a\right\rangle=0
$$

for all $a$ with $a(0)=0=a(\varepsilon)$. Therefore, $b$ satisfies

$$
-\frac{D b}{\partial \tau}-\nabla \operatorname{grad} f(\chi) b=0
$$

on $[0, \varepsilon]$ first in the distribution sense and then in the classical sense by the bootstrap regularity of the ODE (5.14) and so it is smooth.

Let

$$
\begin{aligned}
& P: T_{u_{-}\left(o_{-}\right)} M \rightarrow T_{u_{+}\left(\mathrm{o}_{+}\right)} M, a(0) \rightarrow a(\varepsilon), \\
& P^{\dagger}: T_{u_{-}\left(o_{-}\right)} M \rightarrow T_{u_{+}\left(\mathrm{o}_{+}\right)} M, b(0) \rightarrow b(\varepsilon)
\end{aligned}
$$

be the linear maps for solutions $a, b$ of ODE (5.10) and (5.14), respectively, We note that $P=d \phi_{f}^{\varepsilon}\left(u_{-}\left(o_{-}\right)\right)$. Then for solutions $a, b$ we have

$$
\frac{d}{d \tau}\langle a(\tau), b(\tau)\rangle=\langle\nabla \operatorname{grad} f(\chi) a(\tau), b(\tau)\rangle+\langle a(\tau),-\nabla \operatorname{grad} f(\chi) b(\tau)\rangle=0,
$$

This in particular implies

$$
\left\langle P a(0), P^{\dagger} b(0)\right\rangle=\langle a(\varepsilon), b(\varepsilon)\rangle=\langle a(0), b(0)\rangle .
$$


Substituting (5.14) into the above we obtain

$$
\begin{aligned}
0= & -\left\langle\xi_{-}\left(o_{-}\right), b(0)\right\rangle+\int_{\dot{\Sigma}_{-}}\left\langle\left(D_{u_{-}} \bar{\partial}_{\left(K^{-}, J^{-}\right)}\right)^{\dagger} \eta_{-}, \xi_{-}\right\rangle \\
& \left.+\left\langle\xi_{+}\left(o_{+}\right), b(\varepsilon)\right)\right\rangle+\int_{\dot{\Sigma}_{+}}\left\langle\left(D_{u_{+}} \bar{\partial}_{\left(K^{+}, J^{+}\right)}\right)^{\dagger} \eta_{+}, \xi_{+}\right\rangle .
\end{aligned}
$$

Now we can vary $\xi_{ \pm}$independently and hence we have

$$
\begin{aligned}
& 0=-\left\langle\xi_{-}\left(o_{-}\right), b(0)\right\rangle-\int_{\dot{\Sigma}_{-}}\left\langle\left(D_{u_{-}} \bar{\partial}_{\left(K^{-}, J^{-}\right)}\right)^{\dagger} \eta_{-}, \xi_{-}\right\rangle, \\
& 0=\left\langle\xi_{+}\left(o_{+}\right), b(\varepsilon)\right\rangle+\int_{\dot{\Sigma}_{+}}\left\langle\left(D_{u_{+}} \bar{\partial}_{\left(K^{+}, J^{+}\right)}\right)^{\dagger} \eta_{+}, \xi_{+}\right\rangle
\end{aligned}
$$

and hence

$$
\begin{gathered}
\left(D_{u_{-}} \bar{\partial}_{\left(K^{-}, J^{-}\right)}\right)^{\dagger} \eta_{-}-b(0) \delta_{o_{-}}=0 \\
\left(D_{u_{+}} \bar{\partial}_{\left(K^{+}, J^{+}\right)}\right)^{\dagger} \eta_{+}+b(\varepsilon) \delta_{o_{+}}=0 .
\end{gathered}
$$

Here $\delta_{o}$ denotes the Dirac-delta measure supported at the point $\{o\} \subset \Sigma$. Due to the choice of our metric on the domain $\mathbb{C}$ of $u_{ \pm}, \eta_{ \pm}$must have the singularity of the type $\frac{1}{\bar{z}}$ which is the fundamental solution to $\partial \eta=\vec{b} \delta_{o}$ which lies in $L^{q}$ for any $1<q<2$. Therefore, we can solve the distributional equation

$$
\left(D_{u_{ \pm}} \bar{\partial}_{\left(K^{ \pm}, J^{ \pm}\right)}\right)^{\dagger} \eta=\vec{b} \cdot \delta_{o_{ \pm}}
$$

provided that $\vec{b}$ satisfies the Fredholm alternative:

$$
\left\langle\vec{b}, \xi_{ \pm}\left(o_{ \pm}\right)\right\rangle=\int_{\dot{\Sigma}_{ \pm}}\left\langle\vec{b} \cdot \delta_{o_{ \pm}}, \xi_{ \pm}\right\rangle=0
$$

for all $\xi_{ \pm} \in \operatorname{ker}\left(\left(D_{u_{ \pm}} \bar{\partial}_{\left(K^{ \pm}, J^{ \pm}\right)}\right)^{\dagger}\right)^{\dagger}=\operatorname{ker} D_{u_{ \pm}} \bar{\partial}_{\left(K^{ \pm}, J^{ \pm}\right)}$. Namely $\vec{b} \in\left(V_{ \pm}\right)^{\perp}$, where

$$
V_{ \pm}:=e v_{\mathrm{o} \pm}\left(\operatorname{ker} D_{u_{ \pm}} \bar{\partial}_{\left(K^{ \pm}, J^{ \pm}\right)}\right)
$$

We fix such a solution denoted by $\eta_{\vec{b}} \in L^{q}$.

Then (5.16) can be written as

$$
\left(D_{u_{-}} \bar{\partial}_{\left(K^{-}, J^{-}\right)}\right)^{\dagger}\left(\eta_{-}-\eta_{b(0)}\right)=0, \quad\left(D_{u_{+}} \bar{\partial}_{\left(K^{+}, J^{+}\right)}\right)^{\dagger}\left(\eta_{+}+\eta_{b(\varepsilon)}\right)=0
$$

i.e.,

$$
\begin{aligned}
& \eta_{-}+\eta_{b(0)} \in \operatorname{ker}\left(D_{u_{-}} \bar{\partial}_{\left(K_{-}, J_{-}\right)}\right)^{\dagger} \\
& \eta_{+}-\eta_{b(\varepsilon)} \in \operatorname{ker}\left(D_{u_{+}} \bar{\partial}_{\left(K_{+}, J_{+}\right)}\right)^{\dagger}
\end{aligned}
$$

Therefore, we have the exact sequence

$$
\begin{aligned}
& 0 \rightarrow \operatorname{Graph} P^{\dagger} \cap\left(V_{-}^{\perp} \times V_{+}^{\perp}\right) \stackrel{i}{\rightarrow} \operatorname{ker} E^{\dagger}(u) \\
& \stackrel{j}{\rightarrow} \operatorname{ker}\left(D_{u_{+}} \bar{\partial}_{\left(K_{+}, J_{+}\right)}\right)^{\dagger} \oplus \operatorname{ker}\left(D_{u_{-}} \bar{\partial}_{\left(K_{-}, J_{-}\right)}\right)^{\dagger} \rightarrow 0: .
\end{aligned}
$$


Here the first homomorphism is the map

$$
\mathrm{i}\left(b_{1}, b_{2}\right)=\left(\eta_{b_{1}}, b_{b_{1}},-\eta_{b_{2}}\right)
$$

where $b_{b_{1}}$ is a solution of (5.14) satisfying $b_{b_{1}}(0)=b_{1}$. Note that we have $b_{b_{1}}(\varepsilon)=b_{2}$ if and only if $\left(b_{1}, b_{2}\right) \in$ Graph $P^{\dagger}$. And the second map $j$ is given by

$$
j\left(\eta_{-}, b, \eta_{+}\right)=\left(\eta_{-}+\eta_{b(0)}, \eta_{+}-\eta_{b(\varepsilon)}\right),
$$

and so $\operatorname{ker} E^{\dagger}(u)$ has its dimension given by

$$
\begin{aligned}
\operatorname{dim} \operatorname{ker}\left(D_{u_{+}} \bar{\partial}_{\left(K_{+}, J_{+}\right)}\right)^{\dagger}+\operatorname{dim} \operatorname{ker}\left(D_{u_{-}} \bar{\partial}_{\left(K_{-}, J_{-}\right)}\right)^{\dagger} \\
+\operatorname{dim} \operatorname{Graph} P^{\dagger} \cap\left(V_{+}^{\perp} \times V_{-}^{\perp}\right) \\
=\operatorname{dim} \operatorname{ker}\left(D_{u_{+}}\left(\bar{\partial}_{\left(K_{+}, J_{+}\right)}\right)^{\dagger}+\operatorname{dim} \operatorname{ker}\left(D_{u_{-}} \bar{\partial}_{\left(K_{-}, J_{-}\right)}\right)^{\dagger}\right. \\
\quad+\operatorname{dim}\left(P^{\dagger} \cdot V_{-}^{\perp} \cap V_{+}^{\perp}\right) .
\end{aligned}
$$

Equivalently $E(u)$ has a closed range and its coker $E(u)$ has dimension the same as this. Combining this dimension counting of coker $E(u)$ with that of $\operatorname{ker} E(u)$ in (5.11), we conclude that $E(u)$ is Fredholm and has index given by

Index $E(u)=\operatorname{dim} \operatorname{ker} E(u)-\operatorname{dim} \operatorname{ker} E^{\dagger}(u)$

$$
\begin{aligned}
& =\operatorname{Index} D_{u_{+}} \bar{\partial}_{\left(K^{+}, J^{+}\right)}+\operatorname{Index} D_{u_{-}} \bar{\partial}_{\left(K^{-}, J^{-}\right)}-2 n \\
& =\left(n+\mu_{H_{-}}\left(\left[z_{-}, w_{-}\right]\right)+2 c_{1}\left(A_{-}\right)\right)+\left(n-\mu_{H_{+}}\left(\left[z_{+}, w_{+}\right]\right)+2 c_{1}\left(A_{+}\right)\right) \\
& =\mu_{H_{-}}\left(\left[z_{-}, w_{-}\right]\right)-\mu_{H_{+}}\left(\left[z_{+}, w_{+}\right]\right)+c_{1}\left(A_{-}\right)+c_{1}\left(A_{+}\right) .
\end{aligned}
$$

Here we have used

$$
\begin{aligned}
& \operatorname{dim}\left(P \cdot V_{-}+V_{+}\right)+\operatorname{dim}\left(P^{\dagger} \cdot V_{-}^{\perp} \cap V_{+}^{\perp}\right) \\
& \quad=\operatorname{dim}\left(P \cdot V_{-}+V_{+}\right)+\operatorname{dim}\left(\left(P \cdot V_{-}\right)^{\perp} \cap V_{+}^{\perp}\right) \\
& \quad=\operatorname{dim}\left(P \cdot V_{-}+V_{+}\right)+\operatorname{dim}\left(P \cdot V_{-}+V_{+}\right)^{\perp}=2 n,
\end{aligned}
$$

for the second identity, where $P^{\dagger} V_{-}^{\perp}=\left(P V_{-}\right)^{\perp}$ is due to (5.15), and

$$
\begin{aligned}
& \text { Index } D_{u_{-}} \bar{\partial}_{\left(K_{-}, J_{-}\right)}=\left(n+\mu_{H_{-}}\left(\left[z_{-}, w_{-}\right]\right)+2 c_{1}\left(A_{-}\right)\right), \\
& \text {Index } D_{u_{+}} \bar{\partial}_{\left(K_{+}, J_{+}\right)}=\left(n-\mu_{H_{+}}\left(\left[z_{+}, w_{+}\right]\right)+2 c_{1}\left(A_{+}\right)\right)
\end{aligned}
$$

for the third identity.

In Section 9, we will show that for given generic $J^{ \pm}, f$, there exists some $\varepsilon_{0}>0$, such that for $\varepsilon \in\left(0, \varepsilon_{0}\right]$, every "disk-flow-disk" curves $u \in \mathcal{M}^{\varepsilon}\left(\left[z_{-}, w_{-}\right] ; f ;\left[z_{+}, w_{+}\right] ; A_{ \pm}\right)$is regular, in the sense that $E(u)$ is surjective. So $\mathcal{M}^{\varepsilon}\left(\left[z_{-}, w_{-}\right] ; f ;\left[z_{+}, w_{+}\right] ; A_{ \pm}\right)$is a smooth manifold with dimension equal to the index of $E(u)$ :

$$
\mu_{H_{-}}\left(\left[z_{-}, w_{-}\right]\right)-\mu_{H_{+}}\left(\left[z_{+}, w_{+}\right]\right)+c_{1}\left(A_{-}\right)+c_{1}\left(A_{+}\right)
$$

for generic $J^{ \pm}, f$ and small $\varepsilon$. 
5.2. Nodal Floer trajectories of PSS deformation at $\lambda=0$. This is the middle stage of the construction of the above-mentioned piecewise smooth corbodism at which the "phase transition" of the moduli spaces occurs as $\lambda$ pass through $\lambda=0$. In next subsection, we will give an enhanced version of the corresponding moduli space entering in our construction of the cobordism. The definition of the enhanced moduli space will involve a picture of recently developed symplectic field theory [EGH, BEHWZ] in the Morse-Bott setting. (See [FOOO2] also.)

Let $\dot{\Sigma}_{ \pm}$be two compact surfaces each with one positive puncture (resp. one negative puncture) with analytic coordinates. Let $o_{ \pm} \in \dot{\Sigma}_{ \pm}$a marked point and denote by $(\tau, t)$ with $\pm \tau \geq 0$ the cylindrical chart of $\dot{\Sigma}_{ \pm} \backslash\left\{o_{ \pm}\right\}$ such that $z=e^{ \pm 2 \pi(\tau+i t)}$. We fix periodic orbits $z_{ \pm}$of $H_{ \pm}=H_{ \pm}(t)$ and their liftings $\left[z_{ \pm}, w_{ \pm}\right]$, respectively. We denote

$$
\mathcal{M}_{1}\left(\left(K_{ \pm}, J_{ \pm}\right) ;\left[z_{ \pm}, w_{ \pm}\right]\right)=\left\{\left(u_{ \pm}, o_{ \pm}\right) \mid u_{ \pm} \in \mathcal{M}\left(\left(K_{ \pm}, J_{ \pm}\right) ;\left[z_{ \pm}, w_{ \pm}\right]\right), o_{ \pm} \in \dot{\Sigma}_{ \pm}\right\},
$$

respectively. We have the natural evaluation maps

$$
e v_{ \pm}: \mathcal{M}_{1}\left(\left(K_{ \pm}, J_{ \pm}\right) ;\left[z_{ \pm}, w_{ \pm}\right]\right) \rightarrow M ; \quad e v_{ \pm}\left(u_{ \pm}\right)=u_{ \pm}\left(o_{ \pm}\right) .
$$

The standard nodal Floer trajectories will be the elements in the fiber product

$$
\begin{aligned}
& \mathcal{M}_{1}\left(\left(K_{+}, J_{+}\right) ;\left[z_{+}, w_{+}\right]\right)_{e v_{+}} \times_{e v_{-}} \mathcal{M}_{1}\left(\left(K_{-}, J_{-}\right) ;\left[z_{-}, w_{-}\right]\right) \\
& \quad=\left\{\left(u_{+}, u_{-}\right) \mid u_{ \pm} \in \mathcal{M}\left(\left(K_{ \pm}, J_{ \pm}\right) ;\left[z_{ \pm}, w_{ \pm}\right]\right), u_{+}\left(o_{+}\right)=u_{-}\left(o_{-}\right)\right\} .
\end{aligned}
$$

This is the space that appears in the middle of the "chain homotopy" between $\Psi \circ \Phi$ and the identity map on $\operatorname{HF}(H, J)$ proposed by PiunikhinSalamon-Schwarz in $[\mathbf{P S S}]$. To differentiate this moduli space from the later enhanced version of nodal Floer trajectories that we introduce when the nodal points are immersed, we denote this moduli space by

$$
\mathcal{M}_{\text {stand }}^{\text {nodal }}\left(\left[z_{-}, w_{-}\right],\left[z_{+}, w_{+}\right] ;(K, J)\right) \text {. }
$$

On $U \pm$, using the given analytic coordinates $z=\mathrm{e}^{2 \pi(\tau+\mathrm{i} t)}$, we fix a function

$$
\kappa^{+}(\tau)= \begin{cases}0, & \text { if }|\tau| \leq 1 \\ 1, & \text { if }|\tau| \geq 2\end{cases}
$$

and let $\kappa^{-}(\tau)=\kappa^{+}(-\tau)$. We set $\kappa_{\varepsilon}^{+}(\tau)=\kappa^{+}(\tau-R(\varepsilon)+1)$ and $\kappa_{\varepsilon}^{-}(\tau)=$ $\kappa_{\varepsilon}^{+}(-\tau)$. It is easy to see

$$
\kappa_{\varepsilon}^{+}(\tau)=\left\{\begin{array}{ll}
1, & \text { for } \tau \geq R(\varepsilon)+1, \\
0, & \text { for } \tau \leq R(\varepsilon),
\end{array} \quad \kappa_{\varepsilon}^{-}= \begin{cases}1, & \text { for } \tau \leq-R(\varepsilon)-1 \\
0, & \text { for } \tau \geq-R(\varepsilon)\end{cases}\right.
$$

We then extend these outside the charts $U \pm$ by zero. 
We define $\left(K_{\varepsilon}, J_{\varepsilon}\right)$ to be the obvious pairs

$$
\begin{aligned}
& K_{\varepsilon}(\tau, t, x)= \begin{cases}\kappa_{\varepsilon}^{+}(\tau) \cdot K^{+}(\tau, t, x), & (\tau, t) \in U_{+}, \\
\kappa_{\varepsilon}^{-}(\tau) \cdot K^{-}(\tau, t, x), & (\tau, t) \in U_{-}, \\
0, & z \in \Sigma_{\varepsilon} \backslash U_{+} \cup U_{-},\end{cases} \\
& J_{\varepsilon}^{ \pm}(\tau, t, x)= \begin{cases}J^{\kappa_{\varepsilon}^{+}(\tau)}(t, x), & (\tau, t) \in U_{+}, \\
J_{\varepsilon}^{-}(\tau)(t, x), & (\tau, t) \in U_{-}, \\
J_{0}(x), & z \in \Sigma_{\varepsilon} \backslash U_{+} \cup U_{-}\end{cases}
\end{aligned}
$$

associated to $\kappa_{\varepsilon}^{ \pm}$respectively. Here, we denote a gluing of $\Sigma_{+}$and $\Sigma_{-}$by $\Sigma_{+} \#_{\varepsilon} \Sigma_{-}$(see Definition 12.1, Example 12.1 for details). We then extend these to a constant family outside the charts $U \pm$. Thanks to the cut-off functions $\beta_{ \pm}$, this extension defines a smooth family on $\dot{\Sigma}$. We will vary $R=R(\varepsilon)$ depending on $\varepsilon$, so that we are given a one-parameter family

$$
\dot{\Sigma}_{\varepsilon},\left(K_{\varepsilon}, J_{\varepsilon}\right) .
$$

Here, we would like to emphasize that $K_{\varepsilon} \equiv 0$ in the neck regions of $\Sigma_{\varepsilon}$.

5.3. Moduli space of enhanced nodal Floer trajectories. If we attempt to construct a smooth coordinate chart for the parameterized moduli space of dimension 1 near $\lambda=0$, the resolved Floer trajectories should be related to the "disk-flow-disk" elements. One way is to break the local conformal symmetry of the equation near the node by inserting a small Morse function $\varepsilon f$ with $\varepsilon \rightarrow 0$ at the node. This forces one to study adiabatic degeneration as studied in $[\mathbf{O h} \mathbf{5}, \mathbf{O h} \mathbf{3}, \mathbf{M T}]$ and the relevant gluing problem. What distinguishes this gluing problem from the gluing problem in the standard Gromov-Witten or in the Floer theory is that it glues two configurations in different scales: nodal Floer trajectory $\left(u_{-}, u_{+}\right)$in macroscopic level and local model $u_{0}$ in microscopic level. To find the correct local model, we need to analyze the fine structure of the node in the nodal trajectories. Description of this structure is in order.

First of all, we will need to require that the nodal points are immersed points for both $u_{ \pm}$. We will prove that this requirement holds for a generic choice of $J$. For the moment, we will assume that the nodal points are immersed for both $u_{ \pm}$, and continue with our discussion.

Secondly, we need to enhance the moduli space of standard nodal Floer trajectories by some local models, which are to be implanted at the intersection point $u_{+}\left(o_{+}\right)=u_{-}\left(o_{-}\right)$of $u_{+}$and $u_{-}$.

We first describe the space of local models. Let $H$ be a hyperplane of $\mathbb{C} P^{n}$. We identify $\left(\mathbb{C} P^{n}, H\right)$ with

$$
\mathbb{C} P^{n}=\mathbb{P}\left(1 \oplus \mathbb{C}^{n}\right), \quad H=\mathbb{P}\left(0 \oplus \mathbb{C}^{n}\right)
$$


and $\operatorname{Aut}\left(\mathbb{C} P^{n}, H\right)$ is the set of homothety and translations given by

$$
v \mapsto c v+a ; \mathbb{C}^{n} \rightarrow \mathbb{C}^{n}
$$

with $c \in \mathbb{C}^{*}$ and $a \in \mathbb{C}^{n}$. We define

$$
\begin{aligned}
\widetilde{\mathcal{M}}_{(0 ; 2,0)}\left(\mathbb{C} P^{n}, H ; 2\right):= & \left\{\left(u ; p_{1}, p_{2}\right) \mid u: \Sigma \rightarrow \mathbb{C} P^{n}, u\left(p_{i}\right) \in H, p_{1} \neq p_{2},\right. \\
& \operatorname{deg} u=2, \operatorname{Im} u \not \subset H\}, \\
\mathcal{M}_{(0 ; 2,0)}\left(\mathbb{C} P^{n}, H ; 2\right)= & \widetilde{\mathcal{M}}_{(0 ; 2,0)}\left(\mathbb{C} P^{n}, H ; 2\right) / P S L_{2}(\mathbb{C}) .
\end{aligned}
$$

In terms of the cylindrical coordinates $(s, \Theta)$ of $\mathbb{C}^{n} \backslash\{0\} \cong \mathbb{R} \times S^{2 n-1}$, the above moduli space can be identified with

$$
\begin{aligned}
\widetilde{\mathcal{M}}_{(0 ; 2,0)}^{\mathrm{SFT}}\left(\mathbb{C}^{n} ;(1,1)\right)=: & \left\{u: \dot{\Sigma} \rightarrow \mathbb{C}^{n} \mid u: \dot{\Sigma} \cong S^{1} \times \mathbb{R} \rightarrow \mathbb{C}^{n}\right. \text { is proper, } \\
& \bar{\partial}_{J_{0}} u=0, \lim _{\tau \rightarrow \pm \infty} \Theta \circ u(\tau / 2 \pi, t)=\gamma_{ \pm}(t), \\
& \left.\lim _{\tau \rightarrow \pm \infty} \int u_{\tau}^{*} \lambda=2 \pi\right\} .
\end{aligned}
$$

We then define

$$
\mathcal{M}_{(0 ; 2,0)}^{\mathrm{SFT}}\left(\mathbb{C}^{n} ;(1,1)\right)=\widetilde{\mathcal{M}}_{(0 ; 2,0)}^{\mathrm{SFT}}\left(\mathbb{C}^{n} ;(1,1)\right) / P S L_{2}(\mathbb{C}) .
$$

Here $\lambda$ is the standard contact form on $S^{2 n-1}(1) \subset \mathbb{C}^{n}, \gamma_{ \pm}$closed Reeb orbits of $\lambda$ and $(1,1)$ stands for the multiplicity of the closed Reeb orbits $\gamma_{ \pm}$, respectively, and $u_{\tau}: S^{1} \rightarrow \mathbb{C}^{n}$ is the loop defined by $u_{\tau}(t)=u(\tau, t)$. We recall that $T:=\int \gamma^{*} \lambda$ for a closed Reeb orbit $\gamma$ is the same as its period. We denote by

$$
\widetilde{\mathcal{R}}_{1}(\lambda)=\operatorname{Reeb}^{\min }\left(S^{2 n-1}, \lambda\right)
$$

the set of minimal Reeb orbits of period $2 \pi$. Then the diagonal circle action on $\mathbb{C}^{n}$ induces a free $S^{1}$-action on $\widetilde{\mathcal{R}}_{1}(\lambda)$, which makes and $\widetilde{\mathcal{R}}_{1}(\lambda) \rightarrow \mathbb{C} P^{n-1}$ a principal $S^{1}$-bundle. We then denote by $\mathcal{R}_{1}(\lambda)$ that of unparameterized ones, i.e.,

$$
\mathcal{R}_{1}(\lambda)=\widetilde{\mathcal{R}}_{1}(\lambda) / S^{1} .
$$

Now for the purpose of defining the correct nodal Floer trajectories we need to consider one-jet evaluation maps on both $\widetilde{\mathcal{M}}_{(0 ; 2,0)}^{S F T}\left(\mathbb{C}^{n} ;(1,1)\right)$ and $\widetilde{\mathcal{M}}_{1}\left(K_{\varepsilon}^{-}, J_{\varepsilon}^{-} ;\left[z_{-}, w_{-}\right]\right)$(or $\left.\widetilde{\mathcal{M}}_{1}\left(K_{\varepsilon}^{+}, J_{\varepsilon}^{+} ;\left[z_{+}, w_{+}\right]\right)\right)$. We now explain these evaluation maps.

We now fix a local chart $I=\left\{I_{p}: \mathcal{U}_{p} \rightarrow \mathcal{V}_{p}\right\}$ be a given Darboux family. (See Subsection 8.1 for the definition.) Using $I$ we can identify $\left(T_{p} M, J_{p}, \omega_{p}\right)$ with $\mathbb{C}^{n}$ equipped with the standard Kähler structure. Then we can define the evaluation maps

$$
\widetilde{e v}_{I, \pm}^{\#}: \widetilde{\mathcal{M}}_{(0 ; 2,0)}^{\mathrm{SFT}}\left(\mathbb{C}^{n} ;(1,1)\right) \rightarrow \widetilde{\mathcal{R}}_{1}(\lambda):
$$


By the immersed property of $u_{ \pm}$at $o_{ \pm}$, the limit

$$
\gamma_{ \pm}(t):=\lim _{\tau \pm \infty} u_{\tau}(\tau, t)
$$

defines an element $\widetilde{\mathcal{R}}_{1}\left(S^{1}\left(T_{p} M\right), \lambda_{p}\right) \cong \mathcal{R}_{1}(\lambda)$. Then the map $\widetilde{e v}_{I, \pm}^{\#}$ descends to the map

$$
e v_{I, \pm}^{\#}: \mathcal{M}_{(0 ; 2,0)}^{\mathrm{SFT}}\left(\mathbb{C}^{n} ;(1,1)\right) \rightarrow \mathcal{R}_{1}(\lambda) ; \quad e v_{I, \pm}^{\#}(u)=\left[\gamma_{ \pm}\right]
$$

for $u_{ \pm} \in \widetilde{\mathcal{M}}\left(K_{\varepsilon}^{ \pm}, J_{\varepsilon}^{ \pm} ;\left[z_{ \pm}, w_{ \pm}\right]\right)$. Since we will not change the Darboux family $I$, we often omit the sub-index $I$ as long as there occurs no confusion.

To get rid of the domain automorphism, we consider the moduli space

$$
\left.\widetilde{\mathcal{M}}_{(1 ; 2,0)}^{\mathrm{SFT}}\left(\mathbb{C}^{n} ;(1,1)\right)=\left\{\left(u,\left(e_{ \pm}, r\right)\right)\right) \mid u: \dot{\Sigma} \rightarrow \mathbb{C}^{n}\right\}
$$

and

$$
\mathcal{M}_{(1 ; 2,0)}^{\mathrm{SFT}}\left(\mathbb{C}^{n} ;(1,1)\right)=\widetilde{\mathcal{M}}_{(1 ; 2,0)}^{\mathrm{SFT}}\left(\mathbb{C}^{n} ;(1,1)\right) / \sim
$$

where $e_{ \pm}$are punctures on $\dot{\Sigma}$ and $r$ is a marked point in the interior, $\sim$ is the equivalence relation under the action of $P S L(2, \mathbb{C})$. After modding out by $\operatorname{PSL}(2, \mathbb{C})$, we can identify $\mathcal{M}_{(1 ; 2,0)}^{\mathrm{SFT}}\left(\mathbb{C}^{n} ;(1,1)\right)$ with the more concrete space

$$
\left\{u \mid u: \mathbb{R} \times S^{1} \rightarrow \mathbb{C}^{n}, \bar{\partial}_{J_{0}} u=0,\left[\lim _{\tau \rightarrow \pm \infty} u_{\tau}(\cdot)\right] \in \mathcal{R}_{1}(\lambda)\right\}
$$

via the unique conformal identification

$$
\varphi:(\dot{\Sigma}, r) \cong \mathbb{R} \times S^{1}
$$

such that $\varphi\left(e_{ \pm}\right)= \pm \infty$ and $\varphi(r)=(0,0)$.

We denote the corresponding moduli space also by

$$
\mathcal{M}^{\mathrm{SFT}}\left(\mathbb{R} \times S^{1}, \mathbb{C}^{n} ; \mathcal{R}_{1}(\lambda)\right) \cong \mathcal{M}_{(1 ; 2,0)}^{\mathrm{SFT}}\left(\mathbb{C}^{n} ;(1,1)\right) .
$$

We have the evaluation maps

$$
e v_{\#,-}^{\mathrm{SFT}}: \mathcal{M}_{(1 ; 2,0)}^{\mathrm{SFT}}\left(\mathbb{C}^{n} ;(1,1)\right) \rightarrow \mathcal{R}_{1}(\lambda)
$$

by

$$
e v_{\#,-}^{\mathrm{SFT}}(u)=\left[\lim _{\tau \rightarrow-\infty} u_{\tau}\right] \in \mathcal{R}_{1}(\lambda) .
$$

Similarly, we define $e v_{\#,+}^{\mathrm{SFT}}: \mathcal{M}_{(1 ; 2,0)}^{\mathrm{SFT}}\left(\mathbb{C}^{n} ;(1,1)\right) \rightarrow \mathcal{R}_{1}(\lambda)$.

Lemma 5.1. The above definition of ev $\underset{\#, \pm}{S F T}$ pushes down to the quotient moduli space

$$
\mathcal{M}_{(1 ; 2,0)}^{S F T}\left(\mathbb{C}^{n} ;(1,1)\right) / \operatorname{Aut}\left(\mathbb{C}^{n}\right) .
$$


Proof. We need to prove

$$
e v_{\#}^{\mathrm{SFT}}(u)=e v_{\#}^{\mathrm{SFT}}(g \circ u)
$$

for all $(g: v \mapsto c v+a) \in \operatorname{Aut}\left(\mathbb{C}^{n}\right)$. For the identity for $e v_{\#}^{\mathrm{SFT}}$, we note that the action induced by the elements of $\operatorname{Aut}\left(\mathbb{C}^{n}\right)$ does not change the asymptotic limit $\lim _{\tau \rightarrow \pm \infty} u_{\tau}$ as an unparameterized Reeb orbit. This finishes the proof.

Now we are ready to give the definition of "enhanced" moduli space of nodal Floer trajectories appearing in the PSS picture.

Let $I=\left\{I_{p}: \mathcal{U}_{p} \rightarrow \mathcal{V}_{p}\right\}$ be the given Darboux family. (See Subsection 8.1 for the definition.) This family provides an isomorphism between $T_{p} M$ and $\mathbb{C}^{n}$ at any $p \in M$. We now consider the evaluation maps

$$
\begin{aligned}
& e v_{+, I}^{\#}: \mathcal{M}_{1}\left(K_{\varepsilon}^{+}, J_{\varepsilon}^{+} ;\left[z_{+}, w_{+}\right]\right) \rightarrow \mathcal{R}_{1}(\lambda), \\
& e v_{-, I}^{\#}: \mathcal{M}_{1}\left(K_{\varepsilon}^{-}, J_{\varepsilon}^{-} ;\left[z_{+}, w_{+}\right]\right) \rightarrow \mathcal{R}_{1}(\lambda)
\end{aligned}
$$

as before for $u \in \mathcal{M}_{1}\left(K_{\varepsilon}^{*}, J_{\varepsilon}^{*} ;\left[z_{*}, w_{*}\right]\right)$. We further note that $\mathcal{M}_{1}\left(K_{\varepsilon}^{ \pm}, J_{\varepsilon}^{ \pm}\right.$; $\left.\left[z_{ \pm}, w_{ \pm}\right]\right)$have decomposition

$$
\begin{aligned}
& \mathcal{M}_{1}\left(K_{\varepsilon}^{+}, J_{\varepsilon}^{+} ;\left[z_{+}, w_{+}\right]\right)=\bigcup_{A \in \pi_{2}(M)} \mathcal{M}_{1}\left(K_{\varepsilon}^{+}, J_{\varepsilon}^{+} ;\left[z_{+}, w_{+}\right] ; A\right), \\
& \mathcal{M}_{1}\left(K_{\varepsilon}^{-}, J_{\varepsilon}^{-} ;\left[z_{-}, w_{-}\right]\right)=\bigcup_{A \in \pi_{2}(M)} \mathcal{M}_{1}\left(K_{\varepsilon}^{-}, J_{\varepsilon}^{-} ;\left[z_{-}, w_{-}\right] ; A\right),
\end{aligned}
$$

where $\mathcal{M}_{1}\left(K_{\varepsilon}^{ \pm}, J_{\varepsilon}^{ \pm} ;\left[z_{ \pm}, w_{ \pm}\right] ; A\right)$ are the sets

$$
\begin{aligned}
& \mathcal{M}_{1}\left(K_{\varepsilon}^{+}, J_{\varepsilon}^{+} ;\left[z_{+}, w_{+}\right] ; A_{+}\right) \\
& \quad=\left\{u_{+} \in \mathcal{M}_{1}\left(K_{\varepsilon}^{+}, J_{\varepsilon}^{+} ;\left[z_{+}, w_{+}\right]\right) \mid\left[u \# w_{+}\right]=A_{+} \in \pi_{2}(M)\right\}, \\
& \mathcal{M}_{1}\left(K_{\varepsilon}^{-}, J_{\varepsilon}^{-} ;\left[z_{-}, w_{-}\right] ; A_{-}\right) \\
& \quad=\left\{u_{-} \in \mathcal{M}_{1}\left(K_{\varepsilon}^{-}, J_{\varepsilon}^{-} ;\left[z_{-}, w_{-}\right]\right) \mid\left[\bar{w}_{-} \# u\right]=A_{-} \in \pi_{2}(M)\right\} .
\end{aligned}
$$

We can define the fiber product

$$
\begin{aligned}
& \mathcal{M}_{1}\left(K_{\varepsilon}^{+}, J_{\varepsilon}^{+} ;\left[z_{+}, w_{+}\right] ; A_{+}\right)_{e v_{+, I}^{\#}} \times_{e v_{\#,-}^{\mathrm{SFT}}} \mathcal{M}_{(0 ; 2,0)}^{\mathrm{SFT}}\left(\mathbb{C}^{n} ;(1,1)\right)_{e v_{\#,+}^{\mathrm{SFT}}} \\
& \quad \times_{e v_{-, I}^{\#}} \mathcal{M}_{1}\left(K_{\varepsilon}^{-}, J_{\varepsilon}^{-} ;\left[z_{-}, w_{-}\right] ; A_{-}\right)
\end{aligned}
$$

for each given pair $A_{ \pm} \in \pi_{2}(M)$. Then we form the union

$$
\begin{aligned}
\mathcal{M}^{\text {nodal }}\left(\left[z_{-}, w_{-}\right],\left[z_{+}, w_{+}\right] ;(K, J)\right) & :=\bigcup_{\left(A_{-}, A_{+}\right) ; A_{-}+A_{+}=0} \mathcal{M}_{1}\left(K_{\varepsilon}^{+}, J_{\varepsilon}^{+} ;\left[z_{+}, w_{+}\right] ; A_{+}\right)_{e v_{+, I}^{\#}} \\
& \quad \times_{e v_{\#,-}^{\mathrm{SFT}}} \mathcal{M}_{(0 ; 2,0)}^{\mathrm{SFT}}\left(\mathbb{C}^{n} ;(1,1)\right)_{e v_{\#,+}^{\mathrm{SFT}}} \times_{e v_{-, I}^{\#}} \mathcal{M}_{1}\left(K_{\varepsilon}^{-}, J_{\varepsilon}^{-} ;\left[z_{-}, w_{-}\right] ; A_{-}\right)
\end{aligned}
$$



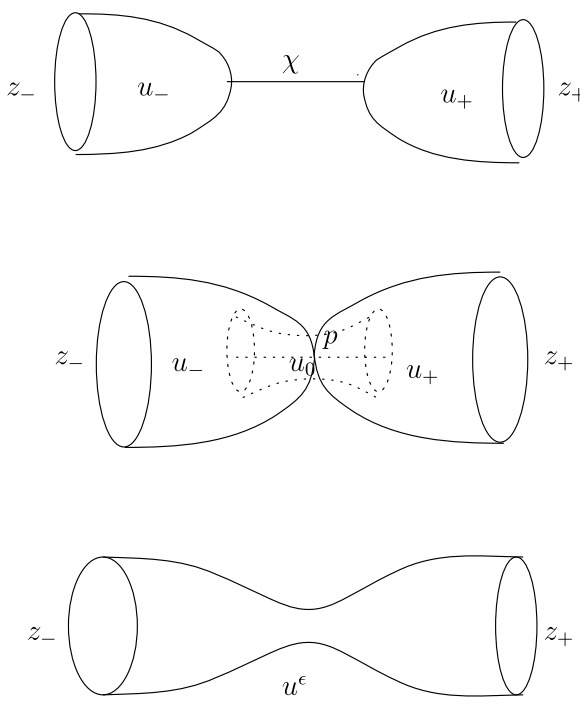

Figure 3. The enhanced PSS scheme.

under the assumption that $d u_{ \pm}\left(o_{ \pm}\right) \neq 0$. We call elements $\left(u_{+}, u_{0}, u_{-}\right)$ therein enhanced nodal Floer trajectories in vacuum (Figure 3). The following theorem justifies the hypothesis that the nodal points are immersed, and so that the above fiber product is well defined. We will postpone its proof to the next subsection.

Theorem 5.1. Let $(K, J ; \varepsilon)$ be a Floer datum with the asymptotic Hamiltonian $H$ such that it satisfies (5.19), (5.20). Suppose that

$$
\mu\left(\left[z_{-}, w_{-}\right]\right)-\mu\left(\left[z_{+}, w_{+}\right]\right)<2 n-1 .
$$

Then there exists a dense subset of $\mathcal{J}_{\omega}$ consisting of $J$ 's such that for any quintuple

$$
\left(u_{-}, u_{+}, r_{-}, r_{+} ; \varepsilon\right) \in \cup_{0<\varepsilon \leq \varepsilon_{0}} \mathcal{M}_{\text {stand }}^{\text {nodal }}\left(\left[z_{-}, w_{-}\right],\left[z_{+}, w_{+}\right] ;\left(K_{\varepsilon}, J_{\varepsilon}\right)\right)
$$

with $u_{-}\left(r_{-}\right)=u_{+}\left(r_{+}\right), r_{-}$and $r_{+}$are immersed points of $u_{-}$and $u_{+}$respectively, and

$$
\left[d u_{-}\left(r_{-}\right)\right] \neq\left[d u_{+}\left(r_{+}\right)\right] \quad \text { in } \mathbb{P}\left(T_{p} M\right)
$$

with $p=u_{-}\left(r_{-}\right)=u_{+}\left(r_{+}\right)$.

In particular, these hold when $\mu\left(\left[z_{-}, w_{-}\right]\right)-\mu\left(\left[z_{+}, w_{+}\right]\right)=0$.

5.4. Nodal points are immersed. In this subsection, we will give the proof of Theorem 5.1. The proof is a variation of the dimension counting argument and partially inspired by Hutchings and Taubes's proof of Theorem $4.1[\mathbf{H u T}]$ in which they studied immersion properties of pseudoholomorphic curves in the symplectization of contact three-manifolds. 
Consider the parameterized family $(K, J)=\left(\left\{K_{\varepsilon}\right\},\left\{J_{\varepsilon}\right\}\right)$ such that

$$
J \equiv J_{0}
$$

in a neighborhood of the marked point $r \in \dot{\Sigma}$. We consider a pair of them denoted by $\left(K_{ \pm}, J_{ \pm}\right)$.

We consider $\left(J,\left(u_{-}, r_{-}\right),\left(u_{+}, r_{+}\right), \varepsilon\right)$ and the map

$$
\begin{aligned}
\Upsilon & :\left(J,\left(u_{-}, r_{-}\right),\left(u_{+}, r_{+}\right), \varepsilon\right) \\
& \mapsto\left(\bar{\partial}_{(J, K)^{-} ; \varepsilon}\left(u_{-}\right), \bar{\partial}_{(J, K)^{+} ; \varepsilon}\left(u_{+}\right) ; \partial_{J} u\left(r_{-}\right), \partial_{J} u\left(r_{+}\right)\right),
\end{aligned}
$$

where we denote

$$
\begin{aligned}
& \bar{\partial}_{(K, J)^{-} ; \varepsilon}\left(u_{-}\right)=\left(d u+P_{K_{\varepsilon}^{-}}\right)_{J_{\varepsilon}^{-}}^{(0,1)}(u), \\
& \bar{\partial}_{(K, J)^{+} ; \varepsilon}\left(u_{+}\right)=\left(d u+P_{K_{\varepsilon}^{+}}\right)_{J_{\varepsilon}^{+}}^{(0,1)}(u) .
\end{aligned}
$$

We consider the bundles over $\Sigma \times M$

$$
\begin{aligned}
& H_{J_{0}}^{(0,1)}(\Sigma \times M):=\cup_{(z, x)} H_{o m}^{\prime \prime}\left(T_{z} \Sigma, T_{x} M\right), \\
& H_{J_{0}}^{(1,0)}(\Sigma \times M):=\cup_{(z, x)} H_{o m}^{\prime} J_{J_{0}}\left(T_{z} \Sigma, T_{x} M\right),
\end{aligned}
$$

whose fibers are $J$-anti-linear and $J$-linear parts of $\operatorname{Hom}\left(T_{z} \Sigma, T_{x} M\right)$, respectively. The union of standard nodal Floer trajectories

$$
\mathcal{M}_{\text {stand }}^{\text {nodal }}\left(\left[z_{-}, w_{-}\right],\left[z_{+}, w_{+}\right] ;(K, J) ; R(\varepsilon)\right)
$$

over $J \in \mathcal{J}_{\omega}$ is nothing but

$$
\begin{aligned}
& \Upsilon^{-1}\left(\{0\} \times\{0\} \times H_{J_{0}}^{(1,0)}(\Sigma \times M) \times{ }_{\Delta} H_{J_{0}}^{(1,0)}(\Sigma \times M)\right) \\
& \quad:=\mathcal{M}_{\text {stand }}^{\text {nodal }}\left(\left[z_{-}, w_{-}\right],\left[z_{+}, w_{+}\right] ; K ; R(\varepsilon)\right) .
\end{aligned}
$$

We recall that $K_{ \pm} \equiv 0$ near the marked points $o_{ \pm}$. Therefore, we have

$$
\bar{\partial}_{(J, K)^{ \pm} ; \varepsilon} u_{ \pm}\left(o_{ \pm}\right)=\bar{\partial}_{J_{0}}\left(u_{ \pm}\right)\left(o_{ \pm}\right)
$$

which implies that for any $u_{ \pm}$with $\bar{\partial}_{(J, K)^{ \pm} ; \varepsilon}\left(u_{ \pm}\right)\left(o_{ \pm}\right)=0$, we have

$$
d u\left(o_{ \pm}\right)=0, \quad \text { if and only if } \partial_{J} u\left(o_{ \pm}\right)=0 .
$$

Postponing the precise functional analytic details until Section 8.3, we introduce the necessary framework for the Fredholm theory needed to prove Theorem 5.1. We denote by

$$
\mathcal{F}^{-}=\mathcal{F}^{-}\left(\dot{\Sigma}, M ;\left[z_{-}, w_{-}\right]\right), \quad \mathcal{F}^{+}=\mathcal{F}^{+}\left(\dot{\Sigma}, M ;\left[z_{+}, w_{+}\right]\right)
$$

the off-shell function space hosting the operator $\bar{\partial}_{(K, J)^{ \pm}}$and the corresponding Floer moduli spaces, respectively. And we introduce the standard bundle

$$
\begin{array}{ll}
\mathcal{H}^{\prime \prime}=\cup_{(u, J)} \mathcal{H}_{(u, J)}^{\prime \prime}, & \mathcal{H}_{(u, J)}^{\prime \prime}=\Omega_{J}^{(0,1)}\left(u^{*} T M\right), \\
\mathcal{H}^{\prime}=\cup_{(u, J)} \mathcal{H}_{(u, J)}^{\prime}, & \mathcal{H}_{(u, J)}^{\prime}=\Omega_{J}^{(1,0)}\left(u^{*} T M\right) .
\end{array}
$$


We have the natural evaluation map

$$
e v_{\mathcal{F}^{*}}: \mathcal{F}^{*} \rightarrow H_{J_{0}}^{(1,0)}(\Sigma \times M) ; \quad e v_{\mathcal{F}^{*}}(u)=\left(o_{*}, u\left(o_{*}\right) ; \partial_{J} u\left(o_{*}\right)\right)
$$

for $*= \pm$, respectively. Then the above map $\Upsilon$ defines a section of the Banach bundle

$$
\mathcal{J}_{\omega} \times \mathcal{F}^{-} \times \mathcal{F}^{+} \rightarrow \mathcal{H}_{-}^{\prime \prime} \times \mathcal{H}_{+}^{\prime \prime} \times H_{J_{0}}^{(1,0)} \times H_{J_{0}}^{(1,0)}
$$

We now prove the following proposition by a standard argument via the linearization of $\Upsilon$. We use the convention that $o_{L}$ is the zero section for a bundle $L$.

Proposition 5.2. The map $\Upsilon$ is transverse to the (stratified) submanifold

$$
\begin{aligned}
& o_{\mathcal{H}_{-}^{\prime \prime}} \times o_{\mathcal{H}_{+}^{\prime \prime}} \times\left(o_{H_{J_{0}}^{(1,0)}} \times{ }_{\Delta} H_{J_{0}}^{(1,0)} \bigcup H_{J_{0}}^{(1,0)} \times{ }_{\Delta} o_{H_{J_{0}}}^{(1,0)}\right) \\
& \subset \mathcal{H}_{-}^{\prime \prime} \times \mathcal{H}_{+}^{\prime \prime} \times H_{J_{0}}^{(1,0)} \times H_{J_{0}}^{(1,0)} .
\end{aligned}
$$

In particular the set

$$
\Upsilon^{-1}\left(o_{\mathcal{H}_{-}^{\prime \prime}} \times o_{\mathcal{H}_{+}^{\prime \prime}} \times\left(o_{H_{J_{0}}^{(1,0)}} \times_{\Delta} H_{J_{0}}^{(1,0)} \bigcup H_{J_{0}}^{(1,0)} \times_{\Delta} o_{H_{J_{0}}^{(1,0)}}\right)\right)
$$

is a (stratified) submanifold of

$$
\mathcal{M}_{\text {stand }}^{\text {nodal }}\left(\left[z_{-}, w_{-}\right],\left[z_{+}, w_{+}\right] ; K ; \varepsilon\right)
$$

of codimension $2 n$.

Proof. It is easy to check the statement on the codimension and so we will focus on proving the submanifold property. We note that the subset

$$
o_{\mathcal{H}_{-}^{\prime \prime}} \times o_{\mathcal{H}_{+}^{\prime \prime}} \times\left(o_{H_{J_{0}}^{(1,0)}} \times_{\Delta} H_{J_{0}}^{(1,0)} \bigcup H_{J_{0}}^{(1,0)} \times_{\Delta} o_{H_{J_{0}}^{(1,0)}}\right)
$$

consists of two strata: one is the open stratum given by

$$
\begin{aligned}
& o_{\mathcal{H}_{-}^{\prime \prime}} \times o_{\mathcal{H}_{+}^{\prime \prime}} \times\left(o_{H_{J_{0}}^{(1,0)}} \times{ }_{\Delta} H_{J_{0}}^{(1,0)} \bigcup H_{J_{0}}^{(1,0)} \times_{\Delta} o_{H_{J_{0}}^{(1,0)}}\right) \backslash \\
& o_{\mathcal{H}_{-}^{\prime \prime}} \times o_{\mathcal{H}_{+}^{\prime \prime}} \times\left(o_{H_{J_{0}}^{(1,0)}} \times{ }_{\Delta} o_{H_{J_{0}}^{(1,0)}}\right)
\end{aligned}
$$

and the other is given by the lower-order stratum

$$
o_{\mathcal{H}_{-}^{\prime \prime}} \times o_{\mathcal{H}_{+}^{\prime \prime}} \times\left(o_{H_{J_{0}}^{(1,0)}} \times{ }_{\Delta} o_{H_{J_{0}}^{(1,0)}}\right) .
$$

We note that the lower-dimensional stratum has codimension $2 n$ insider the set (5.25).

The linearization of $\Upsilon$ is given by

$$
\begin{aligned}
& \left(B,\left(\xi_{-}, v_{-}\right),\left(\xi_{+}, v_{+}\right), h\right) \mapsto\left(D \bar{\partial}_{\left(K_{-}\right)}\left(u_{-}\right)\left(B, \xi_{-}\right), D \bar{\partial}_{\left(K_{+}\right)}\left(u_{+}\right)\left(B, \xi_{+}\right) ;\right. \\
& \quad\left(\xi_{-}\left(o_{-}\right),\left(D \partial_{J_{-}, u_{-}}\left(\xi_{-}\right)\left(o_{-}\right)\right),\left(\xi_{+}\left(o_{+}\right),\left(D \partial_{J_{+}, u_{+}}\left(\xi_{+}\right)\left(o_{+}\right)\right)\right),\right.
\end{aligned}
$$


for $B \in T_{J_{0}} \mathcal{J}_{\omega}, \xi_{ \pm} \in T_{u_{ \pm}} \mathcal{F}^{ \pm}$, and $v_{ \pm} \in T_{o_{ \pm}} \Sigma_{ \pm}$. It is well known that $D \bar{\partial}_{\left(K_{-}\right)}$ $\left(u_{-}\right)\left(B, \xi_{-}\right), D \bar{\partial}_{\left(K_{+}\right)}\left(u_{+}\right)\left(B, \xi_{+}\right)$are surjective and so we will focus on the problem of finite-dimensional transversality of the linear map

$$
\left(\xi_{-}, \xi_{+}\right) \mapsto\left(\left(\xi_{-}\left(o_{-}\right),\left(D \partial_{J_{-}, u_{-}}\left(\xi_{-}\right)\left(o_{-}\right)\right),\left(\xi_{+}\left(o_{+}\right),\left(D \partial_{J_{+}, u_{+}}\left(\xi_{+}\right)\left(o_{+}\right)\right)\right)\right.\right.
$$

to the submanifold

$$
o_{H_{J_{0}}}^{(1,0)} \times{ }_{\Delta} H_{J_{0}}^{(1,0)} \bigcup H_{J_{0}}^{(1,0)} \times{ }_{\Delta} o_{H_{J_{0}}^{(1,0)}}
$$

in $H_{J_{0}}^{(1,0)}(\Sigma \times M) \times H_{J_{0}}^{(1,0)}(\Sigma \times M)$.

Since transversality of the map $\left(u_{-}, u_{+}\right) \mapsto\left(u_{-}\left(o_{-}\right), u_{+}\left(o_{+}\right)\right)$to $\Delta \subset$ $M \times M$ is obvious, we will focus on the other factor on the tangential data. We first consider the top dimensional stratum, i.e., for the pair $\left(u_{-}, u_{+}\right)$ such that $u_{-}\left(o_{-}\right)=u_{+}\left(o_{+}\right)$and

$$
d u_{-}\left(o_{-}\right)=0, \quad d u_{+}\left(o_{+}\right) \neq 0 .
$$

We need to prove that the equation

$$
D \partial_{J, u}\left(\xi_{-}\right)\left(o_{-}\right)=\eta_{-}
$$

has a solution $\xi_{-}$for each given $\eta_{-} \in \Lambda^{(1,0)}(T M)$. Similar consideration applies to the case of switching + and - .

In general, a well-known computation shows

$$
D_{u} \partial_{J}\left(\xi_{-}\right)=\left(\nabla_{u} \xi_{-}\right)_{J}^{(1,0)}+T_{J}^{(1,0)}\left(d u_{-}, \xi\right)
$$

with the torsion term $T$. However, if $d u_{-}\left(o_{-}\right)=0, T_{J}^{(1,0)}\left(d u_{-}\left(o_{-}\right)\right.$, $\left.\xi\left(o_{-}\right)\right)=0$ for any $\xi$.

We now introduce the linear operator $q_{J, x_{0}}$ defined by

$$
q_{J, x_{0}}(x)=\left(J_{x_{0}}+J(x)\right)^{-1}\left(J_{x_{0}}-J(x)\right)
$$

for $x$ such that $d\left(x, x_{0}\right)<\delta$ for $\delta>0$ depending only on $(M, \omega, J)$ but independent of $x_{0} \cdot q_{J, x_{0}}$ satisfies $q_{J, x_{0}}\left(x_{0}\right)=0$. (See [Si].) Then if we identify $\left(T_{x_{0}} M, J_{x_{0}}\right) \cong \mathbb{C}^{n}$, we can write the operator

$$
\left(\nabla_{u} \xi_{-}\right)_{J}^{(1,0)}=\partial \xi_{-}-q_{J, r}(u) \bar{\partial} \xi_{-}+D \cdot \xi_{-}
$$

for some zero-order operator $D$ with $D\left(o_{-}\right)=0$.

Therefore, if $u$ satisfies $d u_{-}\left(o_{-}\right)=0$, we can write

$$
D \partial_{J, u}(\xi)=\partial \xi_{-}-A \cdot \bar{\partial} \xi_{-}+C \cdot \xi_{-}
$$

in a neighborhood of $o_{-}$where $A, C$ are smooth pointwise (matrix) multiplication operators with

$$
A\left(o_{-}\right)=C\left(o_{-}\right)=0 .
$$

To finish the proof, we need to prove the existence of local solutions of the equation

$$
\partial \xi_{-}-A \cdot \bar{\partial} \xi_{-}+C \cdot \xi_{-}=\eta_{-}
$$


near the given point $r_{-}$. This equation can be transformed into

$$
(I d-A \cdot \bar{T}) \partial \xi_{-}+C \cdot \xi_{-}=\eta_{-},
$$

where $T$ is the operator

$$
\begin{aligned}
\bar{T} g(z) & =p \cdot v \cdot\left(\frac{1}{2 \pi \mathrm{i}} \iint_{D} \frac{g(\zeta)}{(\bar{\zeta}-z)^{2}} d \zeta \wedge d \bar{\zeta}\right) \\
& =-\lim _{\delta \rightarrow 0} \iint_{\zeta|| \bar{\zeta}-z|\geq \delta,| \zeta \mid \leq 1} \frac{g(\zeta)}{(\bar{\zeta}-z)^{2}} d \zeta \wedge d \bar{\zeta} .
\end{aligned}
$$

The operator $T$ satisfies the a priori estimate

$$
\|T g\|_{W^{k, p}} \leq A_{k, p}\|g\|_{k, p}
$$

(see $[\mathbf{V e}]$, p. $166-167$ of $[\mathbf{S i}]$ ).

Now after multiplying a cut-off function to $\eta_{-}$with its support contained in a sufficiently small neighborhood of $r_{-}$and using the a priori estimate (for $k=1$ ), we can solve (5.28) by the contraction mapping theorem in a neighborhood of $o_{-}$. This finishes the existence of a solution to (5.26) for the top stratum.

For the lower-dimensional stratum, i.e., for those pairs $\left(u_{-}, u_{+}\right)$with

$$
u_{-}\left(o_{-}\right)=u_{+}\left(\mathrm{o}_{+}\right), \quad d u_{-}\left(\mathrm{o}_{-}\right)=d u_{+}\left(\mathrm{o}_{+}\right)=0
$$

we can prove the existence by the same argument. The only thing to make sure is that the surjectivity proof of $D \bar{\partial}_{(K, J)^{ \pm}}$using the same variation $B$ still holds. But it is easy to check that the nodal condition $u_{-}\left(o_{-}\right)=u_{+}\left(o_{+}\right)$ ensures this, whose checking is left to the readers. (see [OZZ1] for a complete discussion on this matter.)

This finishes the proof of the proposition.

We have the natural projection

$$
\pi_{\Upsilon}: \Upsilon^{-1}\left(o_{\mathcal{H}_{-}^{\prime \prime}} \times o_{\mathcal{H}_{+}^{\prime \prime}} \times\left(o_{H_{J_{0}}^{(1,0)}} \times_{\Delta} H_{J_{0}}^{(1,0)} \bigcup H_{J_{0}}^{(1,0)} \times_{\Delta} o_{H_{J_{0}}^{(1,0)}}\right)\right) \rightarrow \mathcal{J}_{\omega},
$$

which is the restriction of the projection map

$$
\mathcal{M}_{\text {stand }}^{\text {nodal }}\left(\left[z_{-}, w_{-}\right],\left[z_{+}, w_{+}\right] ; K\right) \rightarrow \mathcal{J}_{\omega},
$$

where we denote

$$
\mathcal{M}_{\text {stand }}^{\text {nodal }}\left(\left[z_{-}, w_{-}\right],\left[z_{+}, w_{+}\right] ; K\right)=\bigcup_{J \in \mathcal{J}_{\omega}} \mathcal{M}_{\text {stand }}^{\text {nodal }}\left(\left[z_{-}, w_{-}\right],\left[z_{+}, w_{+}\right] ; K, J\right) .
$$

Since the latter projection has the index $\mu\left(\left[z_{-}, w_{-}\right]\right)-\mu\left(\left[z_{+}, w_{+}\right]\right)+1$ (for the parameterized problem over $\left.0<\varepsilon \leq \varepsilon_{0}\right)$, the Fredholm index of $\pi_{\Upsilon}$ is given by $\mu\left(\left[z_{-}, w_{-}\right]\right)-\mu\left(\left[z_{+}, w_{+}\right]\right)+1-2 n$. 
Therefore for any regular value $J$ of $\pi_{\Upsilon}$, the preimage will be empty whenever

$$
\mu\left(\left[z_{-}, w_{-}\right]\right)-\mu\left(\left[z_{+}, w_{+}\right]\right)<2 n-1
$$

and in particular when $\mu\left(\left[z_{-}, w_{-}\right]\right)-\mu\left(\left[z_{+}, w_{+}\right]\right)=0$ or -1 . This finishes the proof of Theorem 5.1 except the requirement $\left[d u_{-}\left(o_{-}\right)\right] \neq\left[d u_{+}\left(o_{+}\right)\right]$. But this itself can be proved by refining the above genericity argument with an addition of another stratum

$$
\Delta_{H_{J}^{(1,0)}} \subset H_{J}^{(1,0)} \times H_{J}^{(1,0)}
$$

whose details we leave to the readers.

5.5. Resolved nodal Floer trajectories in Morse background. This subsection is the third stage of the deformation of the parameterized moduli space of the PSS cobordism.

In this subsection, we consider the Riemann surface with one positive and one negative punctures

$$
(\Sigma ; p, q)
$$

with analytic charts. Modulo the action of $P S L(2, \mathbb{C})$, we may identify this with the standard cylinder

$$
\left(\mathbb{R} \times S^{1} ;\{-\infty\},\{+\infty\}\right)
$$

with a global conformal coordinates $(\tau, t)$. The coordinate is uniquely defined modulo the linear translations

$$
(\tau, t) \mapsto\left(\tau+\tau_{0}, t+t_{0}\right) .
$$

We provide the analytic charts at the punctures $p, q$ so that they are compatible with this identification. Using this coordinates, we write

$$
K=F(\tau, t) d \tau+H(\tau, t) d t
$$

and require the condition of cylindrical ends

$$
F \equiv 0, H \equiv H(t), \quad \text { at } \pm \infty
$$

for $K$. This condition does not affect under the coordinate change $(\tau, t) \mapsto$ $\left(\tau+\tau_{0}, t+t_{0}\right)$ and so is well-defined.

Similarly we also fix a homotopy from $J_{0}$ to $J(t)\left\{J^{s}\right\}_{0 \leq s \leq 1}$ so that $J^{0}=$ $J_{0}, J^{1}=J$.

We will consider a one-parameter family of such pairs $\left(K_{\varepsilon}, J_{\varepsilon}\right)$ with their cylindrical ends given by

$$
\operatorname{End}_{ \pm}\left(K_{\varepsilon}, J_{\varepsilon}\right)=(H, J), \quad R_{0} \leq R(\varepsilon)<\infty
$$

for a given Floer-regular pair $(H, J)$. For this purpose, we use the family of function $\kappa_{\varepsilon}^{ \pm}$constructed in (5.19) for $R \in \mathbb{R}_{+}=[0, \infty)$. We also define a 
function $\rho_{\varepsilon}: \mathbb{R} \rightarrow[0,1]$ so that

$$
\begin{gathered}
\rho_{\varepsilon}(\tau)= \begin{cases}1, & \text { for }|\tau| \leq R(\varepsilon)-1, \\
0, & \text { for }|\tau| \geq R(\varepsilon),\end{cases} \\
\left|\rho_{\varepsilon}^{\prime}(\tau)\right| \leq 2, \quad \text { for } R(\varepsilon)-1 \leq|\tau| \leq R(\varepsilon) .
\end{gathered}
$$

For the main purpose of the present paper, we will later choose $R=R(\varepsilon)$ so that

$$
\varepsilon R(\varepsilon) \rightarrow 0, \quad \text { as } \varepsilon \rightarrow 0 .
$$

We remark that the choice of $R(\varepsilon)$ made in (5.32) will be needed for some normalization procedure which will be explained later in the course of the adiabatic degeneration argument.

We define $J_{\varepsilon}$ by

$$
J_{\varepsilon}(\tau, t, x)= \begin{cases}J^{\kappa_{\varepsilon}^{+}(\tau)}(t, x), & \text { for } \tau \geq R(\varepsilon), \\ J_{0}(x), & \text { for }|\tau| \leq R(\varepsilon)-1, \\ J^{\kappa_{\varepsilon}^{-}(\tau)}(t, x), & \text { for } \tau \leq-R(\varepsilon) .\end{cases}
$$

Thanks to the cut-off functions $\kappa_{ \pm}$, this defines a smooth $\mathbb{R} \times S^{1}$ family of almost complex structures $J$ on $\mathrm{M}$.

Similarly, we define the family $K_{\varepsilon}: \mathbb{R} \times S^{1} \times M \rightarrow \mathbb{R}$ by

$$
K_{\varepsilon}(\tau, t, x)= \begin{cases}\kappa_{\varepsilon}^{+}(\tau) \cdot H(t, x), & \text { for } \tau \geq R(\varepsilon), \\ \rho_{\varepsilon}(\tau) \cdot \varepsilon f(x), & \text { for }|\tau| \leq R(\varepsilon), \\ \kappa_{\varepsilon}^{-}(\tau) \cdot H(t, x), & \text { for } \tau \leq-R(\varepsilon) .\end{cases}
$$

Remark 5.2. We would like to compare our choice of $K_{\varepsilon}$ above with that of [PSS, MS]: the latter uses a family of $K_{\varepsilon}$ with $K_{\varepsilon} \equiv 0$ in the neck region of $\Sigma_{\varepsilon}$, while we use the one by putting a small Morse function $\varepsilon f$ in the neck and take the adiabatic limit as $\varepsilon \rightarrow 0$. With the choice $K_{\varepsilon} \equiv 0$ in the neck region, this process of degenerating Floer trajectories to nodal ones by letting $\varepsilon \rightarrow 0$ is not at all obvious to the authors. However this process is not properly explained in [PSS, MS].

Now using this particular one-parameter family $\left(K_{R}, J_{R}\right)$ for a given cutoff function $\kappa=\left\{\kappa_{+}, \kappa_{-}\right\}$, we consider the corresponding parameterized moduli space

$$
\begin{aligned}
& \mathcal{M}^{\text {para }}\left(\left[z_{-}, w_{-}\right],\left[z_{+}, w_{+}\right]\right) ;\{(K, J ; \kappa)\} \\
& \quad=\bigcup_{0<\varepsilon \leq \varepsilon_{0}} \mathcal{M}\left(\left[z_{-}, w_{-}\right],\left[z_{+}, w_{+}\right] ; K_{\varepsilon}, J_{\varepsilon}\right) .
\end{aligned}
$$

For the simplicity of notations, we will also write $\mathcal{M}^{\text {para }}=\cup_{0<\varepsilon \leq \varepsilon_{0}} \mathcal{M}^{\varepsilon}$ whenever there is no danger of confusion. To study the map $\Psi \circ \Phi$ in homology, 
we need to analyze compactification of $\mathcal{M}^{\text {para }}$. In the next several sections of the paper, we prove the following theorem.

Theorem 5.2. The parameterized moduli space $\mathcal{M}^{\text {para }}$ as $0<\varepsilon \leq \varepsilon_{0}$ can be compactified to a one-dimensional smooth manifold with boundary whose collar is diffeomorphic to

$$
\left[0, \varepsilon_{0}\right) \times \mathcal{M}_{(0 ; 1,1)}^{\text {nodal }}\left(\left[z_{-}, w_{-}\right],\left[z_{+}, w_{+}\right] ;(H, J),\left(f, J_{0}\right)\right)
$$

for a sufficiently small $\varepsilon_{0}>0$.

This theorem provides the right-hand one-sided collar neighborhood of $\mathcal{M}_{0}$ in $\mathcal{M}^{\text {para }}=\cup_{-\varepsilon_{0} \leq \varepsilon \leq \varepsilon_{0}} \mathcal{M}_{\varepsilon}$ mentioned in Section 4.3.

\section{Local models near nodes in vacuum}

We study proper holomorphic curves in $\mathbb{C}^{n}$ with cylindrical end $\mathbb{R} \times S^{2 n-1}$ with a cylindrical metric on at the ends thereof: we provide a metric $g$ conformal to the standard metric on $\mathbb{C}^{n}$ and has the form

$$
g=d s^{2}+g_{S^{2 n-1}}=\frac{1}{r^{2}} g_{\mathbb{C}^{n}}
$$

at infinity where $(r, \Theta)$ is the standard polar coordinates of $\mathbb{C}^{n} \backslash\{0\}$ and $r=\mathrm{e}^{s}$ for the cylindrical coordinates $(s, \Theta) \in \mathbb{R} \times S^{2 n-1}(1)$.

The standard complex structure on $\mathbb{C}^{n}$ provides an almost complex structure on the cylinder that is translational invariant, and the symplectic form written in the coordinates as

$$
\omega_{0}=d\left(r^{2} \Theta\right)=d\left(e^{2 s} \Theta^{*} \lambda\right)=\mathrm{e}^{2 s}\left(2 d s \wedge \Theta^{*} \lambda+d \Theta^{*} \lambda\right)
$$

where $\lambda$ is the standard contact form on $S^{2 n-1}=S^{2 n-1}(1)$. The set of Reeb orbits of $\lambda$ on $S^{2 n-1}$ consists of the Hopf circles with constant speed which forms a smooth family parameterized by $\mathbb{C} P^{n-1}$ and gives a Morse-Bott type degenerate asymptotic condition at infinity for the relevant pseudoholomorphic curves on $\mathbb{C}^{n}$. A relevant Fredholm theory has been given in $[\mathbf{H W Z 3}]$ in three dimension. And in a general Morse-Bott setting the Fredholm theory has been laid out in [Bou] and [FOOO2].

We modify the exposition given in [FOOO2] in our context. The book [FOOO2] dealt with the more nontrivial case with Lagrangian boundary conditions. Because we cannot directly borrow the results therefrom, we provide detailed explanation in our current context.

6.1. Classification of local models. We note that the unit sphere $S^{2 n-1}$ has the standard contact form given by

$$
\lambda=\frac{1}{2} \sum_{i=1}^{n}\left(x_{i} d y_{i}-y_{i} d x_{i}\right)
$$


and the associated Reeb vector field by

$$
X_{\lambda}=\sum_{i=1}\left(x_{i} \frac{\partial}{\partial y_{i}}-y_{i} \frac{\partial}{\partial x_{i}}\right) .
$$

It follows from the expression of the Reeb vector field $X_{\lambda}$ that the minimal Reeb orbits of $\left(S^{2 n-1}, \lambda\right)$ are given by the curves $\gamma:[0,2 \pi] \rightarrow \mathbb{C}^{n}$ which parameterize a Hopf circle in $S^{2 n-1}$

$$
S^{2 n-1} \cap L \subset S^{2 n-1},
$$

where $L \subset \mathbb{C}^{n}$ is one-dimensional complex subspace. We note that all the Reeb orbits have the same periods $2 \pi$ and are nondegenerate in the MorseBott sense.

We recall that an element $u \in \mathcal{M}_{(0 ; 2,0)}^{\mathrm{SFT}}\left(\mathbb{C}^{n} ;(1,1)\right)$ is assumed to satisfy the convergence

$$
\begin{aligned}
\lim _{\tau \rightarrow \pm \infty} s \circ u(\tau, t) & =\infty, \\
\lim _{\tau \rightarrow \pm \infty} \Theta \circ u(\tau, t) & =\gamma_{ \pm}(t),
\end{aligned}
$$

respectively, for some Reeb orbit $\gamma_{ \pm} \in \widetilde{\mathcal{R}}_{1}(\lambda)$. The following uniqueness result will be important later in the gluing problem.

Proposition 6.1. Fix a hyperplane $H$ in $\mathbb{C} P^{n}$ and two points $x_{0}, x_{\infty} \in H$. Consider a rational curve that is not contained in $H$. Then there exists a unique rational curve passing through $x_{0}, x_{1}$ of degree 2 modulo the action of Aut $\left(\mathbb{C} P^{n} ; H\right)$ which is the group of automorphisms of $\mathbb{C} P^{n}$ fixing $H$.

Proof. It is easy to construct a degree two map $u: S^{2} \rightarrow \mathbb{C} P^{n}$ through any two given points in $\mathbb{C} P^{n}$ and hence there always exists a map $u: S^{2} \rightarrow \mathbb{C} P^{n}$ a holomorphic map of degree 2 satisfying

$$
u(0)=x_{0}, \quad u(\infty)=x_{\infty} ; \quad x_{0}, x_{\infty} \in H \subset \mathbb{C} P^{n} .
$$

We now prove the uniqueness modulo the action of $\operatorname{Aut}\left(\mathbb{C} P^{n} ; H\right)$. Let $u^{\prime}$ be another such curve with the same asymptotic condition. Then the extension of $u^{\prime}$ to $\mathbb{C} P^{n}$ has the condition $u^{\prime}(0)=u(0)$ and $u^{\prime}(\infty)=u(\infty)$ and $u(z) \in \mathbb{C} P^{n} \backslash H \cong \mathbb{C}$. Now we choose a point $x \in C$ with $C=$ Image $u$. Composing $u^{\prime}$ with an element $g \in \operatorname{Aut}\left(\mathbb{C} P^{n} ; H\right)$ and replacing $u^{\prime}$ by $g \circ$ $u^{\prime}$, we may assume that $u$ and $u^{\prime}$ pass through the three distinct points $\left\{x_{0}, x_{\infty}, x\right\}$. We note that as long as $n \geq 3$, we can find a hyperplane $H^{\prime} \subset$ $\mathbb{C} P^{n}$ that contains the three points. Then both $C, C^{\prime}$, which have degree 2 , must be contained in the hyperplane $H^{\prime} \subset \mathbb{C} P^{n}$ containing the three points. Repeating this argument inductively whenever $n \geq 3$, we can reduce the proof to the case $n=2$, i.e., to $\mathbb{C} P^{2}$. From now on, we assume $n=2$.

We choose a point $x \in C \backslash H$, which exists since $C$ is not a line. Let $L$ be a line that is tangent to $C$ at $x$. We note that any irreducible degree two curve 
is immersed (in fact embedded) and the action of $\operatorname{Aut}\left(\mathbb{C} P^{n}, H\right)=\operatorname{Aut}\left(\mathbb{C}^{n}\right)$ preserves the projective tangent line, i.e., the induced map

$$
g_{*}: \mathbb{P}\left(T_{x} \mathbb{C}^{n}\right) \rightarrow \mathbb{P}\left(T_{g(x)} \mathbb{C}^{n}\right) ;[\ell] \mapsto\left[d_{x} g(\ell)\right]
$$

becomes the identity map under the canonical identification of $\mathbb{P}\left(T_{x} \mathbb{C}^{n}\right)=$ $\mathbb{P}\left(\mathbb{C}^{n}\right)=\mathbb{P}\left(T_{g(x)} \mathbb{C}^{n}\right)$.

Therefore there is a well-defined Gauss map

$$
\mathbb{C} P^{1} \rightarrow \mathbb{C} P^{1}=\mathbb{P}\left(\mathbb{C}^{2}\right) ; p \mapsto[d u(p)],
$$

where $[d u(p)]$ is the tangent line at $u(p)$. Since this map is holomorphic which is not constant for a degree 2 curve $u$, it must be surjective. Let $x^{\prime} \in C^{\prime}$ be a point such that $\left[T_{x} C\right]=\left[T_{x^{\prime}} C^{\prime}\right]$ in $\mathbb{P}\left(\mathbb{C}^{2}\right)$. We apply an element $g$ with $g(x)=x^{\prime}$ to the map $u$. Then the map $g \circ u$ passes through the three points $x_{0}, x_{\infty}, x^{\prime}$ and becomes tangent to $C^{\prime}$ at $x^{\prime}$. Finally, if $g(C)=C^{\prime}$ already, we are finished. Otherwise $\left(C \backslash C^{\prime}\right) \backslash H \neq \emptyset$. We choose a point $y \in\left(C \backslash C^{\prime}\right) \backslash H$ and consider the line $L^{\prime}$ through $x^{\prime}$ and $y$. This line cannot coincide with the tangent line $\left[T_{x^{\prime}} C^{\prime}\right]$ of $C^{\prime}$ and so it must intersect with another point $y^{\prime} \in C^{\prime}$ because $C^{\prime}$ has degree 2 . Now we apply a scaling at the center $x^{\prime}$

$$
g_{\lambda}: y \mapsto x^{\prime}+\lambda\left(y-x^{\prime}\right) ; \mathbb{C}^{2} \rightarrow \mathbb{C}^{2},
$$

which satisfies $g_{\lambda}(y)=y^{\prime}$ (and $\left.g_{\lambda}\left(x^{\prime}\right)=x^{\prime}\right)$. We consider the map $g_{\lambda} \circ g \circ u$ and $u^{\prime}$. They share 4 points and a common tangent at the point $x^{\prime}$. But any two such degree two curves, i.e., conics must coincide up to reparameterization (see, e.g., Remark 4.2.1 in Chapter V $[\mathbf{H a}]$ ). This finishes the proof.

We now derive the following uniqueness result from the above proposition.

Theorem 6.1. For each given $\gamma_{ \pm} \in \mathcal{R}_{1}(\lambda)$, there exists a unique proper holomorphic map $u: \mathbb{R} \times S^{1} \rightarrow \mathbb{C}^{n}$ modulo the action of

$$
\operatorname{Aut}_{l m d}=\operatorname{Aut}\left(\mathbb{C}^{n}\right)
$$

with the given asymptotic condition

$$
e v_{\#, \pm}^{S F T}(u)=\gamma_{ \pm}
$$

Proof. The case where $\gamma_{+}=\gamma_{-}$can be regarded as the limiting case of unique intersection of the curve and $H$ with multiplicity two and can be treated similarly. Therefore we will assume $\gamma_{+} \neq \gamma_{-}$as an unparameterized curve.

Recall that there is a one-one correspondence between $\mathcal{M}_{0 ; 2,0}^{\mathrm{SFT}}\left(\mathbb{C}^{n}\right.$; $(1,1))$ and $\mathcal{M}_{(0,2,0)}\left(\mathbb{C} P^{n}, H ; 2\right)$. The theorem immediately follows from Proposition 6.1.

Remark 6.1. We can be more explicit by giving the equations of rational curves in $\mathbb{C} P^{2}$ with asymptote $x_{+}$and $x_{-}$on the hyperplane $H_{\infty}$. In affine charts $\mathbb{C}=\mathbb{C} P^{1}-\infty \subset \mathbb{C} P^{1}$ and $\mathbb{C}^{2}=\mathbb{C} P^{2}-H_{\infty} \subset \mathbb{C} P^{2}$ the quadratic 
curve satisfies equation $A x^{2}+B x y+C y^{2}+D x+E y+F=0$, where the coefficients $A, \ldots, F$ are in $\mathbb{C}$. For generic coefficients, the equation can be factorized as

$$
\left(k_{+}(x-e)+l_{+}(y-f)\right)\left(k_{-}(x-e)+l_{-}(y-f)\right)=1
$$

for suitable $k_{ \pm}, l_{ \pm}, e$ and $f \in \mathbb{C}$. We rewrite the above equation in a parameterized form

$$
\left\{\begin{array}{l}
k_{+}(x-e)+l_{+}(y-f)=z, \\
k_{-}(x-e)+l_{-}(y-f)=\frac{1}{z}
\end{array}\right.
$$

and solve

$$
\left\{\begin{array}{l}
x(z)=a z+b / z+e \\
y(z)=c z+d / z+f
\end{array}\right.
$$

where $a, \ldots, f$ are in $\mathbb{C}$. Since $z=\mathrm{e}^{2 \pi(\tau+\mathrm{i} t)}$ for $(\tau, t) \in \mathbb{R} \times S^{1}$,

$$
\left\{\begin{array}{l}
\lim _{\tau \rightarrow+\infty}[x(z), y(z), 1]=[a, c, 0]=x_{+} \in H_{\infty}, \\
\lim _{\tau \rightarrow-\infty}[x(z), y(z), 1]=[b, d, 0]=x_{-} \in H_{\infty} .
\end{array}\right.
$$

From (10.3) we can determine the coefficients in (6.3), up to the ambiguity of $e, f$ arising from Aut $\left(\mathbb{C} P^{2}, H_{\infty}\right)$ and the ambiguity of the ratio $a / b$ arising from $\operatorname{Aut}\left(\mathbb{R} \times S^{1}\right)=\mathbb{C}^{*}$. Since any two $\mathbb{C} P^{2}$ in $\mathbb{C} P^{n}$ are related by a projective linear transform, which restricted on the affine chart $\mathbb{C} P^{2} \backslash H_{\infty}$ is a linear transform, from (6.3) we get the equation for the degree 2 curves in the above theorem:

$$
u(z)=A z+B / z+C, \quad z=e^{2 \pi(\tau+i t)} \in \mathbb{C}_{*}, A, B, C \in \mathbb{C}^{n}, A \neq 0, B \neq 0,
$$

for $z \in \mathbb{C}^{*} \cong \mathbb{R} \times S^{1}$.

Remark 6.2 (Local models with Morse background). From the expression (6.5) we can calculate that the center of mass of the loop $u\left(\left\{\tau_{0}\right\} \times S^{1}\right)$ in $\mathbb{C}^{n}$ (with respect to the standard metric) for any $\tau_{0}$ is always at the fixed vector $C \in \mathbb{C}^{n}$ (by mean value theorem of harmonic functions), which indicates that the needed local model is not quite those lying in $\mathcal{M}_{0,2,0}^{\mathrm{SFT}}\left(\mathbb{C}^{n} ;(1,1)\right)$ because they do not reflect the background gradient flow of the given Morse function $f$. This motivates us to look for some models in Section 7 for which the center of mass of the loop $u\left(\{\tau\} \times S^{1}\right)$ resembles the straight line $a \tau$ in $\mathbb{C}^{n} \cong T_{p} M$ where $a=\nabla f(p)$ the gradient vector of $f$ at $p$. 
6.2. Fredholm theory of local models. We can improve the asymptotic property of such constructed holomorphic curves in the following way. For each $\gamma \in \mathcal{R}_{1}(\lambda)$, we have the following exponential convergence statement, which is the analog to the similar results from $[\mathbf{B E H W Z}]$ and can be proved in the same way as other cases of Morse-Bott-Floer theory. (See also section 62 [FOOO2] for the relevant discussion.)

Proposition 6.2. Fix any $p>2$. Let $\varepsilon>0$ be sufficiently small. Consider a holomorphic map $u: \dot{\Sigma} \rightarrow \mathbb{C}^{n}$ satisfying (10.3) and write $u(\tau, t)=$ $(s(\tau, t), \Theta(\tau, t))$ near $\tau=\infty$. Then there exist constants $\tau_{0}$ and $C_{k}, c_{k}>0$ depending only on $k, p$ such that

$$
\begin{array}{r}
\left|\nabla^{k}\left(s(\tau, t)-2 \pi\left(\tau-\tau_{0}\right)\right)\right| \leq C_{k} \mathrm{e}^{\frac{-2 \pi c_{k}|\tau|}{p}}, \\
\left|\nabla^{k}(\Theta(\tau, t)-\gamma(2 \pi t))\right| \leq C_{k} \mathrm{e}^{\frac{-2 \pi c_{k}|\tau|}{p}} .
\end{array}
$$

Proposition 6.2 dictates the adequate function space for the proper Fredholm theory of the pseudo-holomorphic curves in our problem, which we now explain. Let $\delta<2 \pi$ be a positive number.

Our metric $h$ is conformal to the standard Euclidean metric $|d z|^{2}$ on $\mathbb{C}$ such that

$$
h=\lambda(z)|d z|^{2} .
$$

where $\lambda: \mathbb{C} \rightarrow \mathbb{R}$ is a positive radial function such that

$$
\lambda(z)=\frac{1}{|z|^{2}}
$$

when $|z|$ sufficiently large. We also fix a metric $g_{\text {cyl }}$ on $\mathbb{C}^{n}$ conformal to the standard metric $|d w|^{2}$ so that

$$
g_{\text {cyl }}=\mu(w)|d w|^{2}
$$

for a radial function $\mu$ and it becomes the cylindrical metric on the end of $\mathbb{C}^{n}$, i.e.,

$$
\mu(w)=\frac{1}{|w|^{2}}
$$

when $r=\sum_{i=1}^{n}\left|w_{i}\right|^{2}$ is sufficiently large.

With respect to these metrics on the domain and the target, we now define the space

$$
W_{\delta,(0 ; 2,0)}^{1, p}\left(\dot{\Sigma}, \mathbb{C}^{n} ; \gamma_{+}, \gamma_{-}, \tau_{+}, \tau_{-}\right)
$$

for each fixed $\gamma_{ \pm}$in $\mathcal{R}_{1}(\lambda)$ and $\tau_{ \pm} \in \mathbb{R}$ as follows.

Definition 6.1. $W_{\delta,(0 ; 2,0)}^{1, p}\left(\dot{\Sigma}, \mathbb{C}^{n} ; \gamma_{+}, \gamma_{-}, \tau_{+}, \tau_{-}\right)$is the set of all $u=(s \circ$ $u, \Theta \circ u)$ such that

(1) $u \in W_{\mathrm{loc}}^{1, p}$, 
(2) Using coordinate $(\tau, t)$ at the ends of $\dot{\Sigma}, u$ satisfies

$$
\mathrm{e}^{\frac{2 \pi \delta|\tau|}{p}}\left|\Theta_{ \pm}(\tau, t)-\gamma_{ \pm}(t)\right| \in W^{1, p}\left([0, \infty) \times S^{1}, \mathbb{R}\right)
$$

where $\Theta_{ \pm}=\left.\Theta \circ u\right|_{D_{ \pm}}$

(3) and

$$
\mathrm{e}^{\frac{2 \pi \delta|\tau|}{p}}\left|s_{ \pm}(\tau, t)-2 \pi\left(\tau-\tau_{ \pm}\right)\right| \in W^{1, p}\left([0, \infty) \times S^{1}, \mathbb{R}\right),
$$

where $s_{ \pm}=\left.s \circ u\right|_{D_{ \pm}}$.

Here we denote by $D_{ \pm}$the given coordinate disks associated to the analytic charts at $p_{ \pm}$, and use the cylindrical metrics $h=\lambda(|z|)|d z|^{2}$ to define $W^{1, p}$ and the metric $g_{\text {cyl }}$ (6.8) of $\mathbb{C}^{n}$ to define $\mid$. We call the tuple $\left(\left(\gamma_{-}, \tau_{-}\right),\left(\gamma_{+}, \tau_{+}\right)\right)$the asymptotic datum of $u$ relative to the cylindrical ends associated to the given analytic charts.

Proposition 6.2 implies

$$
\mathcal{M}_{(0 ; 2,0)}\left(\dot{\Sigma}, \mathbb{C}^{n}\right) \subset \bigcup_{\gamma_{ \pm} \in \mathcal{R}_{1}(\lambda)} \bigcup_{\tau_{ \pm} \in \mathbb{R}} W_{\delta,(0 ; 2,0)}^{1, p}\left(\dot{\Sigma}, \mathbb{C}^{n} ; \gamma_{ \pm}, \tau_{ \pm}\right)
$$

We define

$$
\left.W_{\delta,(0 ; 2,0)}^{1, p}\left(\dot{\Sigma}, \mathbb{C}^{n}\right)=\bigcup_{\gamma_{ \pm} \in \mathcal{R}_{1}(\lambda)} \bigcup_{\tau_{0} \in \mathbb{R}} W_{\delta,(0 ; 2,0)}^{1, p}\left(\dot{\Sigma}, \mathbb{C}^{n} ; \gamma_{ \pm}, \tau_{ \pm}\right)\right)
$$

We recall that we have the natural projection

$$
\pi: \mathcal{R}_{1}(\lambda) \rightarrow \mathbb{C} P^{n-1}
$$

forms a principal $S^{1}$-bundle with the $S^{1}$-action being the Hopf action, which can also be realized by with the rotations of the domain circle. We denote by

$$
e v_{ \pm}: W_{\delta,(0 ; 2,0)}^{1, p}\left(\dot{\Sigma}, \mathbb{C}^{n}\right) \rightarrow \mathcal{R}_{1}(\lambda)
$$

the evaluation map defined $e v_{ \pm}(u)=u( \pm \infty, \cdot)$.

The following can be proved by a standard argument. We refer to [FOOO2] for the relevant proof in the more complicated context of proper holomorphic curves with Lagrangian boundary conditions. Since we will need to use the description of the tangent space thereof, we give an outline of the proof of this lemma.

Lemma 6.1. $W_{\delta,(0 ; 2,0)}^{1, p}\left(\dot{\Sigma}, \mathbb{C}^{n}\right)$ has the structure of Banach manifold such that the obvious projection

$$
\begin{aligned}
& \left(\left(\pi \circ e v_{+}, e v_{\mathbb{R}}^{+}\right),\left(\pi \circ e v_{-}, e v_{\mathbb{R}}^{-}\right)\right): W_{\delta,(0 ; 2,0)}^{1, p}\left(\dot{\Sigma}, \mathbb{C}^{n}\right) \\
& \quad \rightarrow\left(\mathbb{C} P^{n-1} \times \mathbb{R}\right) \times\left(\mathbb{C} P^{n-1} \times \mathbb{R}\right)
\end{aligned}
$$

defines a locally trivial fiber bundle. 
Proof. The tangent space of this Banach manifold can be constructed as in Lemma 29.5 or Lemma 60.10 [FOOO2]. See [Bou] for a relevant discussion.

We take a smooth function $\chi_{+}:[0, \infty) \rightarrow[0,1]$ such that $\chi_{+}(\tau)=1$ for $\tau>2, \chi_{+}(\tau)=0$ for $\tau<1$, and $\left|\chi_{+}^{\prime}(\tau)\right|<2$. Symmetrically, we let $\chi_{-}(\tau)=\chi_{+}(-\tau)$. Let $u \in W_{\delta,(0 ; 2,0)}^{1, p}\left(\dot{\Sigma}, \mathbb{C}^{n} ; \gamma_{ \pm}, \tau_{ \pm}\right) \subset W_{\delta,(0 ; 2,0)}^{1, p}\left(\dot{\Sigma}, \mathbb{C}^{n}\right)$. We consider the set of all quintuples $\left(U, V_{\mathcal{R}_{1}(\lambda)}^{ \pm}, V_{\mathbb{R}}^{ \pm}\right)$satisfying

(1) $V_{\mathcal{R}_{1}(\lambda)}^{ \pm}(t) \in T_{\gamma_{ \pm}} \mathcal{R}_{1}(\lambda), V_{\mathbb{R}}^{ \pm} \in \mathbb{R} \cong T_{\tau_{ \pm}} \mathbb{R}$, respectively.

(2) $U \in W_{\text {loc }}^{1, p}\left(\dot{\Sigma} ; u^{*} T \mathbb{C}^{n}\right)$.

(3) Denote

$$
\widetilde{U}(\tau, t)=U(\tau, t)-\chi_{-}(\tau) \operatorname{Pal}_{u(\tau, t)} U(-\infty, t)-\chi_{+}(\tau) \operatorname{Pal}_{u(\tau, t)} U(+\infty, t),
$$

where $U(+\infty, t)=\left(V_{\mathbb{R}}^{+}, V_{\mathcal{R}_{1}(\lambda)}^{+}(t)\right)$ in the cylindrical end of $\left(\mathbb{C}^{n}, g_{\text {cyl }}\right)$, and $\mathrm{Pal}_{u(\tau, t)} U(+\infty, t)$ is the parallel transport of $U(+\infty, t)$ from $u(+\infty, t)$ to $u(\tau, t)$ along the minimal geodesic of $\left(\mathbb{C}^{n}, g_{\text {cyl }}\right)$. Similarly, we define $\operatorname{Pal}_{u(\tau, t)} U(+\infty, t)$. Then we have

$$
\mathrm{e}^{\frac{2 \pi \delta|\tau|}{p}}|\widetilde{U}(\tau, t)| \in W_{D_{ \pm}}^{1, p}(\dot{\Sigma}, \mathbb{R}) .
$$

Here we regard $V_{\mathcal{R}_{1}(\lambda)}^{ \pm}$to be a vector field on $\gamma_{ \pm}$in $\mathbb{C}^{n}$. Since every parameterized simple Reeb orbit $\gamma$ in $S^{2 n-1} \subset \mathbb{C}^{n}$ satisfies $\gamma(t)=\mathrm{e}^{ \pm 2 \pi \mathrm{i}} \gamma(0)$, we have $V_{\mathcal{R}_{1}(\lambda)}^{ \pm}(t)=\mathrm{e}^{ \pm 2 \pi \mathrm{i}} V_{\mathcal{R}_{1}(\lambda)}^{ \pm}(0)$.

Let $C^{0}(u)$ be the set of all such quintuples. It becomes a Banach space with norm $\|\cdot\|_{1, p, \delta}$ such that

$$
\begin{aligned}
& \left\|\left(U, V_{\mathcal{R}_{1}(\lambda)}^{ \pm}, V_{\mathbb{R}}^{ \pm}\right)\right\|_{1, p, \delta}^{p}=\left\|\mathrm{e}^{\frac{2 \pi \delta|\tau|}{p}} \widetilde{U}(\tau, t)\right\|_{W^{1, p}}^{p} \\
& +\left|V_{\mathcal{R}_{1}(\lambda)}^{-}(0)\right|^{p}+\left|V_{\mathbb{R}}^{-}\right|^{p}+\left|V_{\mathcal{R}_{1}(\lambda)}^{+}(0)\right|^{p}+\left|V_{\mathbb{R}}^{+}\right|^{p} .
\end{aligned}
$$

We remark that $V_{\mathcal{R}_{1}(\lambda)}^{ \pm}, V_{\mathbb{R}}^{ \pm}$are determined from $U$ in case the norm $\left\|\left(U, V_{\mathcal{R}_{1}(\lambda)}^{ \pm}, V_{\mathbb{R}}^{ \pm}\right)\right\|_{1, p, \delta}$ is finite.

It is standard to check that $W_{\delta,(0 ; 2,0)}^{1, p}\left(\dot{\Sigma}, \mathbb{C}^{n}\right)$ is a Banach manifold and

$$
C^{0}(u)=T_{u} W_{\delta,(0 ; 2,0)}^{1, p}\left(\dot{\Sigma}, \mathbb{C}^{n}\right) .
$$

To show that (6.9) is a locally trivial fiber bundle, we use the $U(n)$ action as a biholomorphic isometry on $\mathbb{C}^{n}$, which preserves the contact form $\left(S^{2 n-1}, \lambda\right)$. It induces an $U(n)$-action on $W_{\delta,(0 ; 2,0)}^{1, p}\left(\dot{\Sigma}, \mathbb{C}^{n}\right)$. Then the map $(6.9)$ is $U(n)$ equivariant.

On the other hand, the group $\mathbb{C}^{*} \cong \operatorname{Aut}\left(\mathbb{C}^{1} ;\{0, \infty\}\right)$ acts on $W_{\delta,(0 ; 1,0)}^{1, p}$ $\left(\dot{\Sigma}, \mathbb{C}^{n}\right)$ as the automorphism of the domain and on $\mathbb{C} P^{n-1} \times \mathbb{R}$ trivially on $\mathbb{C} P^{n-1}$ and by an addition by $\frac{1}{2 \pi} \ln |z|$ on $\mathbb{R}$. 
Then (6.9) is $\mathbb{C}^{*}$-equivariant. The local triviality (6.9) follows from this equivariance.

We next put

$$
C^{1}(u)=L_{\delta}^{p}\left(\dot{\Sigma}, \Lambda^{(0,1)}\left(u^{*} T \mathbb{C}^{n}\right)\right) .
$$

Then there exists an infinite-dimensional vector bundle over $W_{\delta,(0 ; 2,0)}^{1, p}\left(\dot{\Sigma}, \mathbb{C}^{n}\right)$ whose fiber at $u$ is $C^{1}(u)$.

The formal linearization of the Cauchy-Riemann operator $\bar{\partial}$ defines an operator

$$
D_{u} \bar{\partial}: C^{0}(u) \rightarrow C^{1}(u) .
$$

We apply $D_{u} \bar{\partial}$ only the first component $U$ of the triple $\left(U, V_{\mathcal{R}_{1}(\lambda)}, V_{\mathbb{R}}\right)$.

To see $D_{u} \bar{\partial}$ indeed maps to $C^{1}(u)$, consider the function

$$
\tilde{u}(\tau, t)=\mathrm{e}^{2 \pi\left(\tau-\tau_{+}\right)} \gamma_{+}(t)=\mathrm{e}^{2 \pi\left(\tau-\tau_{+}+\sqrt{-1} t\right)} \gamma_{+}(0)
$$

in $\mathbb{C}^{n}$, then $\tilde{u}$ is holomorphic, and has the same asymptote as $u$ when $\tau \rightarrow$ $+\infty$. It is easy to verify that

$$
D_{\tilde{u}} \bar{\partial}\left(P a l_{u(\tau, t)} U(+\infty, t)\right)=0 .
$$

Using (6.12) the fact that $u-\tilde{u} \in W_{\delta}^{1, p}\left([0,+\infty] \times S^{1}\right)$, we see

$$
D_{u} \bar{\partial}\left(P a l_{\tilde{u}(\tau, t)} U(+\infty, t)\right) \in L_{\delta}^{p}\left([0,+\infty) \times S^{1}\right),
$$

similarly

$$
\left.D_{u} \bar{\partial}\left(P a l_{\tilde{u}(\tau, t)} U(-\infty, t)\right) \in L_{\delta}^{p}(-\infty, 0] \times S^{1}\right) .
$$

Hence $\left(D_{u} \bar{\partial}\right)(U)$ is contained in $C^{1}(u)$.

Proposition 6.3. The operator (6.11) is Fredholm with index given by

$$
\text { Index } D_{u} \bar{\partial}=4(n+1)+4-6+1=4 n+3 \text {. }
$$

Proof. The Fredholm property can be proved in the same way as Lemma 60.14 [FOOO2] and so its proof is omitted referring readers thereto. The index formula can be derived from the general theory from [Bou, EGH] but we will give its proof as a consequence of the classification, Proposition 6.1, and the transversality of the local models, which we will prove in the next subsection.

6.3. Transversality of local models. We recall that each element $u \in$ $\mathcal{M}_{(0 ; 2,0)}\left(\dot{\Sigma}, \mathbb{C}^{n}\right)$ has the convergence

$$
\lim _{\tau \rightarrow \pm \infty} s \circ u(\tau, t)=\infty, \quad \lim _{\tau \rightarrow \pm \infty} \Theta \circ u(\tau, t)=\gamma_{ \pm}(t)
$$

for some $\gamma_{ \pm} \in \mathcal{R}_{1}(\lambda)$. 
By Proposition 6.2, we have

$$
\mathcal{M}_{(0 ; 2,0)}\left(\dot{\Sigma}, \mathbb{C}^{n}\right) \subset W_{\delta,(0 ; 2,0)}^{1, p}\left(\dot{\Sigma}, \mathbb{C}^{n}\right)
$$

We first prove the following surjectivity.

Proposition 6.4. Let $u \in \mathcal{M}_{(0 ; 2,0)}\left(\dot{\Sigma}, \mathbb{C}^{n}\right)$ and

$$
C^{0}(u)=T_{u} W_{\delta,(0 ; 2,0)}^{1, p}\left(\dot{\Sigma}, \mathbb{C}^{n}\right), \quad C^{1}(u)=L_{\delta}^{p}\left(\dot{\Sigma}, \Lambda^{(0,1)}\left(u^{*} T \mathbb{C}^{n}\right)\right) .
$$

Then the linearization operator

$$
D_{u} \bar{\partial}: C^{0}(u) \rightarrow C^{1}(u)
$$

is surjective.

Proof. We note that since the almost complex structure on $\mathbb{C}^{n}$ is integrable, we have

$$
D_{u} \bar{\partial}=\text { the standard Dolbeault operator. }
$$

Here we recall from Theorem 5.1 that the nodal points are immersed and the tangent planes of the two components at the nodes are different. This is translated as the local model $u$ corresponds to a degree two rational curve in $\mathbb{P}^{n}$ that intersect the hyperplane $\mathbb{H}$ at two distinct points transversely. Furthermore since we use the cylindrical metrics on both on the domain $\dot{\Sigma}$ and $\mathbb{C}^{n}$, the operator

$$
D_{u} \bar{\partial}: C^{0}(u) \rightarrow C^{1}(u)
$$

can be shown to be conjugate to the standard Dolbeault operator

$$
\bar{\partial}: W^{1, p}\left(u^{*} T \mathbb{P}^{n}\right) \rightarrow L^{p}\left(\Lambda_{J}^{(0,1)}\left(u^{*} T \mathbb{P}^{n}\right)\right)
$$

if we regard $u$ as a map from $\mathbb{P}^{1}$ to $\mathbb{P}^{n}$. Once we have these, the surjectivity immediately follows from the well-known fact $H^{1}\left(u^{*} T \mathbb{P}^{n}\right)=\{0\}$ for any rational curve $u: \mathbb{P}^{1} \rightarrow \mathbb{P}^{n}$.

Due to the Morse-Bott character of our gluing problem, this surjectivity will not be enough for our purpose. We need to augment this by the asymptotic evaluation datum at infinity. We recall that ev $: W_{\delta,(0 ; 2,0)}^{1, p}\left(\dot{\Sigma}, \mathbb{C}^{n}\right) \rightarrow$ $\left(\mathbb{C} P^{n-1} \times \mathbb{R}\right) \times\left(\mathbb{C} P^{n-1} \times \mathbb{R}\right)$ is the assignment of the asymptotic datum followed by the projection $\mathcal{R}_{1}(\lambda) \rightarrow \mathbb{C} P^{n-1}$.

The following is the main result of this subsection.

Theorem 6.2. Let $u$ be a holomorphic curve in $\mathcal{M}_{(0 ; 2,0)}\left(\dot{\Sigma}, \mathbb{C}^{n}\right)$ with the asymptotic datum $\left(\gamma_{ \pm}, \tau_{ \pm}\right)$. Then the operator

$D_{u} \bar{\partial} \oplus D \pi: C^{0}(u) \rightarrow C^{1}(u) \oplus T_{\left(\left[\gamma_{-}\right], \tau_{-}\right)}\left(\mathbb{C} P^{n-1} \times \mathbb{R}\right) \oplus T_{\left(\left[\gamma_{+}\right], \tau_{+}\right)}\left(\mathbb{C} P^{n-1} \times \mathbb{R}\right)$ is surjective. 
Proof. We note that the action of $U(n)$ on $\mathbb{C}^{n}$ preserves $\left(S^{2 n-1}, \lambda\right)$ and induces an action on $\mathbb{C} P^{n-1}$. By this $U(n)$-invariance of the equation, it suffices to consider the case when $\gamma_{-}$is the equator given by

$$
\gamma_{-}(t)=(\cos (2 \pi t)+i \sin (2 \pi t), 0, \ldots, 0) \in S^{2 n-1} \subset \mathbb{C}^{n} .
$$

Furthermore, $\mathbb{C} P^{n-1}$ is two-point homogeneous in that any two pair of distinct points can be mapped to each other by the action of $U(n)$ (or more precisely by the action of $P U(n))$.

As a first step, we will establish the following splitting:

$$
C^{0}(u)=T_{u} W_{\delta,(0 ; 2,0)}^{1, p}\left(\dot{\Sigma}, \mathbb{C}^{n}\right)=\mathbb{C}_{-}^{n}(u) \oplus \mathbb{C}_{+}^{n}(u) \oplus W^{1, p}\left(u^{*} T \mathbb{C}^{n}\right)
$$

such that the restriction

$$
D \pi: \mathbb{C}_{-}^{n}(u) \oplus \mathbb{C}_{+}^{n}(u) \rightarrow T_{\left(\left[\gamma_{-}\right], \tau_{-}\right)}\left(\mathbb{C} P^{n-1} \times \mathbb{R}\right) \oplus T_{\left(\left[\gamma_{+}\right], \tau_{+}\right)}\left(\mathbb{C} P^{n-1} \times \mathbb{R}\right)
$$

is surjective.

First, we find a subspace $\mathbb{C}^{n-1} \subset u(n)$ such that

$$
\mathbb{C}^{n-1} \oplus u(n-1)=u(n),
$$

where $U(n-1)$ is the isotropy subgroup of the vector $(1,0, \ldots, 0)$. We identify $\mathbb{C}^{n-1} \cong u(n) / u(n-1)$ with $\{0\} \oplus \mathbb{C}^{n-1} \subset \mathbb{C}^{n}$. The action

$$
A \in \mathbb{C}^{n-1} \cong u(n) / u(n-1) \mapsto A \cdot u
$$

defines an embedding

$$
\mathbb{C}^{n-1} \rightarrow C^{0}(u)=T_{u} W_{\delta,(0 ; 2,0)}^{1, p}\left(\dot{\Sigma}, \mathbb{C}^{n}\right) .
$$

We denote by $\mathbb{C}_{-}^{n-1}(u)$ the image of this embedding.

Similarly, we consider the isotropy group $U(n-1)_{\gamma_{+}}$of $\left[\gamma_{+}\right]$and define an embedding of $\mathbb{C}^{n-1}$ into $C^{0}(u)$ by the similar way. We denote this by $\mathbb{C}_{+}^{n-1}(u)$. Using the fact that $\mathbb{C} P^{n-1}$ is two-point homogeneous, we can choose this embedding so that $\mathbb{C}_{+}^{n-1}(u)$ and $\mathbb{C}_{-}^{n-1}(u)$ are linearly independent. We note by construction of $\mathbb{C}_{ \pm}^{n-1}(u)$ that they are transverse to the Hopf action.

Finally we take the generators $X$ of $\mathbb{C} \cong \operatorname{aut}(\dot{\Sigma}) \cong \operatorname{Aut}(\mathbb{C},\{0\})$ and consider the action

$$
X \mapsto \mathcal{L}_{X} u
$$

This action at each end $\gamma_{ \pm}$of $u$ defines an embedding $\mathbb{C} \rightarrow C^{0}(u)$ which coincides with the infinitesimal action of the translations along the direction of $\tau$ in the analytic charts chosen at $p_{ \pm}$. The image of this embedding is linearly independent of $\mathbb{C}_{ \pm}^{n-1}(u)$. This gives rise to the required splitting by setting

$$
\mathbb{C}_{ \pm}^{n}(u)=\mathbb{C} \dot{\gamma}_{ \pm} \oplus \mathbb{C}_{ \pm}^{n-1}(u),
$$

respectively. By construction, it follows that the map (6.15) is surjective and so we have established the required splitting (6.14). 
By the splitting (6.14), Theorem 12.2 will follow from the surjectivity of the linear map

$$
D_{u} \bar{\partial}: C^{0}(u) \rightarrow C^{1}(u),
$$

which is precisely the content of Proposition 6.4. This finishes the proof.

Once we have established the surjectivity of the linearization operator $D_{u} \bar{\partial}$, the moduli space $\mathcal{M}_{(0 ; 2,0)}\left(\dot{\Sigma}, \mathbb{C}^{n}\right)$ becomes a smooth manifold whose tangent space can be identified with the kernel of the operator $D_{u} \bar{\partial}: C^{0}(u) \rightarrow C^{1}(u)$ for a holomorphic curve $u: \dot{\Sigma} \rightarrow \mathbb{C}^{n}$ found in the uniqueness Theorem 6.1.

The classification theorem, Proposition 6.1, immediately proves the following theorem. This in particular computes the index of $D_{u} \bar{\partial}$, when combined with the surjectivity.

Theorem 6.3. Assume $0<\delta<2 \pi$ is sufficiently small. Let $u$ be the pseudoholomorphic map constructed in Theorem 6.1 associated to the Reeb orbits $\gamma_{ \pm}$. Then we have

$$
\operatorname{dim} \operatorname{Ker} D_{u} \bar{\partial}=4 n+3
$$

and all the elements $u \in \widetilde{\mathcal{M}}_{(0 ; 1,1)}\left(\dot{\Sigma}, \mathbb{C}^{n}\right)$ is transverse and so is a smooth manifold of dimension $6 n$. Furthermore, the quotient space

$$
\widetilde{\mathcal{M}}_{(0 ; 1,1)}\left(\dot{\Sigma}, \mathbb{C}^{n}\right) / \text { Aut }_{l m d}
$$

is a one-point set.

We would like to separately state the following obvious corollary of Theorems 12.2 and 6.3 .

Corollary 6.1. We have

$$
\text { Index } D_{u} \bar{\partial}=4 n+3 \text {. }
$$

\section{Local models near nodes in Morse background}

In this section, we provide the Banach manifold for the solutions $(u, a)$ of the inhomogeneous Cauchy-Riemann equation $\bar{\partial}_{J_{0}} u=a$ in $\left(\mathbb{C}^{n}, J_{0}\right)$, with the same asymptotic condition (10.3) for $u$ at infinity as the homogeneous case in the previous section. Here $u: \mathbb{R} \times S^{1} \rightarrow \mathbb{C}^{n}$ is a map, $\bar{\partial}_{J_{0}}$ is the standard Cauchy-Riemann operator, $a$ is a section of $\Lambda^{0,1}\left(u^{*}\left(T \mathbb{C}^{n}\right)\right)$ that satisfies

$$
a\left(\frac{\partial}{\partial \tau}\right)=\nabla f(p)
$$

in $\mathbb{C}^{n}$ in coordinates $(\tau, t)$, where $z=\mathrm{e}^{2 \pi(\tau+\mathrm{i} t)}$. Then the equation $\bar{\partial} u=a$ is nothing but

$$
\left(\frac{\partial}{\partial \tau}+J_{0} \frac{\partial}{\partial t}\right) u(\tau, t)=\nabla f(p)
$$


whose inhomogeneous term is a constant vector $\nabla f(p)$ in $\left(T_{p} M, J_{p}\right) \cong\left(\mathbb{C}^{n}\right.$, $\left.J_{0}\right)$. We remark that the norm of a section of $\Lambda^{0,1}\left(u^{*}\left(T \mathbb{C}^{n}\right)\right)$ is induced from the asymptotically cylindrical metric $\lambda(x) g_{s t}$ rather than the standard metric $g_{s t}$ in $\mathbb{C}^{n}$, so the norm of the one-form a depends on $u(\tau, t)$ pointwise and is not a constant.

We need to adapt the local models found in the previous section because the ambient manifold has the background gradient flow associated to the given Morse function $f$, which affects the Hamiltonian perturbation $K$ for the resolved nodal Floer trajectories. The relevant rescaling procedure of the Floer equation at the nodes does not yield the homogeneous equation $\bar{\partial} u=0$ but yields the inhomogeneous Cauchy-Riemann equation $\bar{\partial} u=a$ on $T_{p} M \cong \mathbb{C}^{n}$ for the one-form $a$ with $a\left(\frac{\partial}{\partial \tau}\right)=\nabla f(p)$.

Recall the off-shell Banach manifold for homogeneous local models was

$$
W_{\delta,(0 ; 2,0)}^{1, p}\left(\dot{\Sigma}, \mathbb{C}^{n}\right)=W_{\delta,(0 ; 2,0)}^{1, p}\left(\dot{\Sigma}, \mathbb{C}^{n} ;(1,1)\right) .
$$

For inhomogeneous local models, we set the Banach manifold to be the set

$$
\mathcal{B}_{0}:=W_{\delta,(0 ; 2,0)}^{1, p}\left(\dot{\Sigma}, \mathbb{C}^{n}\right) \oplus \mathbb{C}^{n} \backslash\{0\}
$$

of the pair $(u, a)$ and the Banach bundle over it as

$$
\mathcal{L}_{0}:=\bigcup_{(u, a) \in \mathcal{B}_{0}} L_{\delta}^{p}\left(\dot{\Sigma}, \Lambda^{0,1}\left(u^{*} T \mathbb{C}^{n}\right)\right) .
$$

We define the augmented Cauchy Riemann operator $\widehat{\partial}: \mathcal{B}_{0} \rightarrow \mathcal{L}_{0}$ as

$$
\widehat{\partial}: W_{\delta,(0 ; 2,0)}^{1, p}\left(\dot{\Sigma}, \mathbb{C}^{n}\right) \oplus \mathbb{C}^{n} \backslash\{0\} \rightarrow \bigcup_{(u, a) \in \mathcal{B}_{0}} L_{\delta}^{p}\left(\dot{\Sigma}, \Lambda^{0,1}\left(u^{*} T \mathbb{C}^{n}\right)\right)
$$

such that

$$
\widehat{\partial}(u, a)=\bar{\partial} u-a
$$

The following proposition gives the relation between homogeneous and inhomogeneous Cauchy-Riemann equations:

Proposition 7.1. We equip both $\dot{\Sigma}$ and $\mathbb{C}^{n}$ with metrics cylindrical at infinity. Then the followings hold:

(1) $u_{0}$ in $W_{\delta,(0 ; 2,0)}^{1, p}\left(\dot{\Sigma}, \mathbb{C}^{n}\right)$ if and only if $u:=u_{0}+a \tau$ is in $W_{\delta,(0 ; 2,0)}^{1, p}\left(\dot{\Sigma}, \mathbb{C}^{n}\right)$.

(2) $\bar{\partial} u_{0}=0$ if any only if $\bar{\partial} u-a=0$.

(3) Suppose $u_{0}$ in $W_{\delta,(0 ; 2,0)}^{1, p}\left(\dot{\Sigma}, \mathbb{C}^{n}\right)$ has the decomposition $u_{0}=\left(s \circ u_{0}\right.$, $\left.\Theta \circ u_{0}\right)$ that satisfies the asymptotic condition

$$
\begin{aligned}
\lim _{\tau \rightarrow \pm \infty} \Theta \circ u_{0}(\tau, t) & =\gamma_{ \pm}(t), \\
\lim _{\tau \rightarrow \pm \infty} s \circ u_{0}(\tau, t) & =2 \pi\left(\tau-\tau_{ \pm}\right) .
\end{aligned}
$$

Then $u=u_{0}+a \tau$ is also in $W_{\delta,(0 ; 2,0)}^{1, p}\left(\dot{\Sigma}, \mathbb{C}^{n}\right)$ and satisfies the same asymptotics. 
Proof. Since $\bar{\partial}(a \tau)=a$, it follows that $\widehat{\partial}(u, a)=0$ if and only if $\bar{\partial} u_{0}=0$. (1) is a conclusion of (3), by applying (3) to $u=u_{0}+a \tau$ and $u_{0}=u-a \tau$. So it remains to prove the statement $(3)$.

Write $u_{0}(\tau, t)=\left(\Theta_{0}(\tau, t), s_{0}(\tau, t)\right)$ in the cylindrical end of $\mathbb{C}^{n}$. Then by the definition of $W_{\delta,(0 ; 2,0)}^{1, p}\left(\dot{\Sigma}, \mathbb{C}^{n}\right)$, there exist $\gamma_{ \pm} \in \mathcal{R}_{1}(\lambda), \tau_{ \pm} \in \mathbb{R}$ such that

$$
\begin{aligned}
& \mathrm{e}^{\frac{2 \pi \delta|\tau|}{p}}\left|\Theta_{0}(\tau, t)-\gamma_{ \pm}(t)\right|_{S^{2 n-1}} \in W^{1, p}\left(\mathbb{R} \times S^{1}, \mathbb{R}\right), \\
& \mathrm{e}^{\frac{2 \pi \delta|\tau|}{p}}\left|s_{0}(\tau, t)-2 \pi\left(\tau-\tau_{ \pm}\right)\right| \in W^{1, p}\left(\mathbb{R} \times S^{1}, \mathbb{R}\right) .
\end{aligned}
$$

Since $W^{1, p}\left([k, k+1] \times S^{1}\right) \hookrightarrow C^{0, \alpha}\left([k, k+1] \times S^{1}\right)$ when $p>2$, we have

$$
\begin{aligned}
& \max _{\tau \in[k, k+1]} \mathrm{e}^{\frac{2 \pi \delta|\tau|}{p}}\left|\Theta_{0}(\tau, t)-\gamma_{ \pm}(t)\right| \\
& \quad \leq C\left\|\mathrm{e}^{\frac{2 \pi \delta|\tau|}{p}}\left|\Theta_{0}(\tau, t)-\gamma_{ \pm}(t)\right|\right\|_{W^{1, p}\left([k, k+1] \times S^{1}, \mathbb{R}\right)} \rightarrow 0
\end{aligned}
$$

Hence

$$
\left|\Theta_{0}(\tau, t)-\gamma_{ \pm}(t)\right| \leq C \mathrm{e}^{-\frac{2 \pi \delta|\tau|}{p}}
$$

for large enough $\tau$. Similarly,

$$
\left|s_{0}(\tau, t)-2 \pi\left(\tau-\tau_{ \pm}\right)\right| \leq C \mathrm{e}^{-\frac{2 \pi \delta|\tau|}{p}}
$$

for large enough $\tau$. So for large $\tau$,

$$
s_{0}(\tau, t) \geq 2 \pi\left(\tau-\tau_{ \pm}\right)-1
$$

Thus,

$$
\left|u_{0}(\tau, t)\right|=\left|\mathrm{e}^{s_{0}(\tau, t)}\right| \geq \mathrm{e}^{-1} \mathrm{e}^{2 \pi\left|\tau-\tau_{ \pm}\right|} \geq C \mathrm{e}^{2 \pi|\tau|}
$$

for $|\tau|$ sufficiently large.

Let $u(\tau, t)=u_{0}(\tau, t)+a \tau$, and write $u(\tau, t)=(\Theta(\tau, t), s(\tau, t))$. Then

$$
\begin{aligned}
\Theta(\tau, t) & =\frac{u_{0}(\tau, t)+a \tau}{\left\|u_{0}(\tau, t)+a \tau\right\|}=\frac{u_{0}(\tau, t)}{\left\|u_{0}(\tau, t)\right\|} \cdot \frac{\left\|u_{0}(\tau, t)\right\|}{\left\|u_{0}(\tau, t)+a \tau\right\|}+\frac{a \tau}{\left\|u_{0}(\tau, t)+a \tau\right\|} \\
& =\Theta_{0}(\tau, t)\left(1-\frac{\left\|u_{0}(\tau, t)+a \tau\right\|-\left\|u_{0}(\tau, t)\right\|}{\left\|u_{0}(\tau, t)+a \tau\right\|}\right)+\frac{a \tau}{\left\|u_{0}(\tau, t)+a \tau\right\|} .
\end{aligned}
$$

Therefore

$$
\begin{aligned}
\left\|\Theta(\tau, t)-\Theta_{0}(\tau, t)\right\| & \leq \frac{\|a \tau\|}{\left\|u_{0}(\tau, t)+a \tau\right\|}+\frac{\|a \tau\|}{\left\|u_{0}(\tau, t)+a \tau\right\|} \\
& \leq \frac{2\|a\| \tau}{C \mathrm{e}^{2 \pi|\tau|}-\|a\| \tau} \\
& \leq C_{1}\|a\| \mathrm{e}^{-2 \pi|\tau|}
\end{aligned}
$$

for large enough $|\tau|$. Hence

$$
\mathrm{e}^{\frac{2 \pi \delta|\tau|}{p}}\left|\Theta(\tau, t)-\Theta_{0}(\tau, t)\right| \in L^{p}\left(\mathbb{R} \times S^{1}, \mathbb{R}\right) .
$$


Similar straightforward computation also shows

$$
\mathrm{e}^{\frac{2 \pi \delta|\tau|}{p}}\left|\nabla \Theta(\tau, t)-\nabla \Theta_{0}(\tau, t)\right| \in L^{p}\left(\mathbb{R} \times S^{1}, \mathbb{R}\right) .
$$

Hence

$$
\mathrm{e}^{\frac{2 \pi \delta|\tau|}{p}}\left|\Theta(\tau, t)-\Theta_{0}(\tau, t)\right| \in W^{1, p}\left(\mathbb{R} \times S^{1}, \mathbb{R}\right) .
$$

Since

$$
\mathrm{e}^{\frac{2 \pi \delta|\tau|}{p}}\left|\Theta(\tau, t)-\gamma_{ \pm}(t)\right| \leq \mathrm{e}^{\frac{2 \pi \delta|\tau|}{p}}\left(\left|\Theta(\tau, t)-\Theta_{0}(\tau, t)\right|+\left|\Theta_{0}(\tau, t)-\gamma_{ \pm}(t)\right|\right)
$$

we get

$$
\mathrm{e}^{\frac{2 \pi \delta|\tau|}{p}}\left|\Theta(\tau, t)-\gamma_{ \pm}(t)\right| \in W^{1, p}\left(\mathbb{R} \times S^{1}, \mathbb{R}\right) .
$$

Next, we estimate $s(\tau, t)=\log \left|u_{0}(\tau, t)+a \tau\right|$ :

$$
\begin{aligned}
\left|s(\tau, t)-s_{0}(\tau, t)\right| & =\log \frac{\left\|u_{0}(\tau, t)+a \tau\right\|}{\left\|u_{0}(\tau, t)\right\|} \\
& =\log \left(1+\frac{\left\|u_{0}(\tau, t)+a \tau\right\|-\left\|u_{0}(\tau, t)\right\|}{\left\|u_{0}(\tau, t)\right\|}\right) .
\end{aligned}
$$

Since

$$
\left|\frac{\left\|u_{0}(\tau, t)+a \tau\right\|-\left\|u_{0}(\tau, t)\right\|}{\left\|u_{0}(\tau, t)\right\|}\right| \leq \frac{\|a \tau\|}{\left\|u_{0}(\tau, t)\right\|} \leq \frac{\|a \tau\|}{C_{1} \mathrm{e}^{2 \pi|\tau|}}=C_{2}|\tau| \mathrm{e}^{-2 \pi|\tau|} \rightarrow 0,
$$

and $\log (1+h) \sim h$ when $h \rightarrow 0$, for large enough $\tau$

$$
\left|s(\tau, t)-s_{0}(\tau, t)\right| \leq 2 C_{2}|\tau| \mathrm{e}^{-2 \pi|\tau|} .
$$

Now

$$
\mathrm{e}^{\frac{2 \pi \delta|\tau|}{p}}\left|s(\tau, t)-2 \pi\left(\tau-\tau_{ \pm}\right)\right| \leq \mathrm{e}^{\frac{2 \pi \delta|\tau|}{p}}\left(\left|s(\tau, t)-s_{0}(\tau, t)\right|+\left|s_{0}(\tau, t)-2 \pi\left(\tau-\tau_{ \pm}\right)\right|\right),
$$

hence

$$
\mathrm{e}^{\frac{2 \pi \delta|\tau|}{p}}\left|s(\tau, t)-2 \pi\left(\tau-\tau_{ \pm}\right)\right| \in L^{p}\left(\mathbb{R} \times S^{1}, \mathbb{R}\right) .
$$

We also have

$$
\begin{aligned}
\left|\nabla s-\nabla s_{0}\right|= & \left|\frac{(u+a \tau) \cdot(\nabla(u+a \tau))}{|u+a \tau|^{2}}-\frac{u \cdot \nabla u}{|u|^{2}}\right| \\
\leq & |(u+a \tau) \cdot(\nabla(u+a \tau))| \cdot\left|\frac{1}{|u+a \tau|^{2}}-\frac{1}{|u|^{2}}\right| \\
& +\frac{1}{|u|^{2}}|(u+a \tau) \cdot \nabla(u+a \tau)-u \cdot \nabla u| \\
\leq & C_{3}\left(|u||\nabla u| \frac{|a \tau|}{|u|^{3}}+\frac{1}{|u|^{2}}(|a \cdot u|+|a \tau \cdot \nabla u|)\right) \\
\leq & C_{3}|\tau| e^{-2 \pi|\tau|},
\end{aligned}
$$


when $|\tau|$ large. So

$$
\mathrm{e}^{\frac{2 \pi \delta|\tau|}{p}}\left|s(\tau, t)-s_{0}(\tau, t)\right| \in W^{1, p}\left(\mathbb{R} \times S^{1}, \mathbb{R}\right) .
$$

Together with

$$
\mathrm{e}^{\frac{2 \pi \delta|\tau|}{p}}\left|s_{0}(\tau, t)-2 \pi\left(\tau-\tau_{ \pm}\right)\right| \in W^{1, p}\left(\mathbb{R} \times S^{1}, \mathbb{R}\right),
$$

by triangle inequality

$$
\mathrm{e}^{\frac{2 \pi \delta|\tau|}{p}}\left|s(\tau, t)-2 \pi\left(\tau-\tau_{ \pm}\right)\right| \in W^{1, p}\left(\mathbb{R} \times S^{1}, \mathbb{R}\right) .
$$

This finishes the proof of $(3)$.

Denote the moduli space of solutions of $\widehat{\partial}(u, a)=0$ by $\mathcal{M}_{(0 ; 2,0)}^{+}\left(\dot{\Sigma}, \mathbb{C}^{n}\right.$; $(1,1)$ ), which is the moduli space of inhomogeneous local models. By Proposition 7.1 , we see

Corollary 7.1. $\mathcal{M}_{(0 ; 2,0)}^{+}\left(\dot{\Sigma}, \mathbb{C}^{n} ;(1,1)\right) \cong \mathcal{M}_{(0 ; 2,0)}\left(\dot{\Sigma}, \mathbb{C}^{n} ;(1,1)\right) \oplus \mathbb{C}^{n} \backslash\{0\}$.

By the same argument, it is immediate to check the following lemma whose proof is omitted.

Lemma 7.1. $\widehat{\partial}(u, a)=\bar{\partial} u-a$ is indeed in $L_{\delta}^{p}\left(\dot{\Sigma}, \Lambda^{0,1}\left(u^{*} T \mathbb{C}^{n}\right)\right)$.

Next we consider the tangent space of any $(u, a) \in \mathcal{B}_{0}$. The tangent space consists of elements $\left(U, V_{\mathbb{R}^{ \pm}}, V_{\mathcal{R}_{1}^{ \pm}(\lambda)}, h\right)$, where $U$ is a section in $W_{\delta}^{1, p}(\dot{\Sigma}$, $\left.u^{*} T \mathbb{C}^{n}\right), V_{\mathbb{R}^{ \pm}} \in T_{\tau_{ \pm}} \mathbb{R} \cong \mathbb{R}, V_{\mathcal{R}_{1}(\lambda)} \in T_{\gamma_{ \pm}(0)} S^{2 n-1}$, and $h \in T_{a} \mathbb{C}^{n}$. The linearized $\widehat{\partial}$ operator is

$$
\begin{aligned}
& D_{(u, a)} \widehat{\partial}: W_{\delta}^{1, p}\left(\dot{\Sigma}, u^{*} T \mathbb{C}^{n}\right) \oplus T_{a} \mathbb{C}^{n} \rightarrow L_{\delta}^{p}\left(\dot{\Sigma}, \Lambda^{0,1}\left(u^{*} T \mathbb{C}^{n}\right)\right), \\
& D_{(u, a)} \widehat{\partial}\left(U, V_{\mathbb{R}_{ \pm}}, V_{R(\lambda)}, h\right)=D_{u} \bar{\partial} U-h=\bar{\partial} U-h .
\end{aligned}
$$

The last identity holds because $D_{u} \bar{\partial}$ becomes the standard Dolbeault operator in $\mathbb{C}^{n}$. Recall the projection

$$
\begin{aligned}
\pi: W_{\delta,(0 ; 2,0)}^{1, p}\left(\dot{\Sigma}, \mathbb{C}^{n} ;(1,1)\right) \rightarrow\left(S^{2 n-1} \times \mathbb{R}\right) \times\left(S^{2 n-1} \times \mathbb{R}\right), \\
u \rightarrow\left(\gamma_{+}(0), \tau_{+}\right) \times\left(\gamma_{-}(0), \tau_{-}\right), \\
D \pi: T_{u} W_{\delta,(0 ; 2,0)}^{1, p}\left(\dot{\Sigma}, \mathbb{C}^{n}\right) \rightarrow T_{\gamma_{+}(0), \tau_{+}}\left(S^{2 n-1} \times \mathbb{R}\right) \times T_{\gamma_{-}(0), \tau_{-}}\left(S^{2 n-1} \times \mathbb{R}\right) .
\end{aligned}
$$

We consider the combined operator

$$
\begin{aligned}
D_{u} \widehat{\partial} \oplus D \pi: T_{u} W_{\delta,(0 ; 2,0)}^{1, p}\left(\dot{\Sigma}, \mathbb{C}^{n}\right) \rightarrow & L_{\delta}^{p}\left(\dot{\Sigma}, \Lambda^{0,1}\left(u^{*} T \mathbb{C}^{n}\right)\right) \\
& \oplus T_{\left(\gamma_{+}(0), \tau_{+}\right)}\left(S^{2 n-1} \times \mathbb{R}\right) \\
& \oplus T_{\left(\gamma-(0), \tau_{-}\right)}\left(S^{2 n-1} \times \mathbb{R}\right), \\
\left(U, V_{\mathbb{R}_{ \pm}}, V_{\mathcal{R}_{1}(\lambda)}, h\right) \rightarrow & \left(D \bar{\partial} U-h, V_{\mathbb{R}^{+}}^{+}, V_{\mathcal{R}_{1}(\lambda)}^{+}, V_{\mathbb{R}}^{-}, V_{\mathcal{R}_{1}(\lambda)}^{-}\right) .
\end{aligned}
$$


Proposition 7.2. There exists a constant $\eta>0$ depending on $u$ but independent of a such that for $u=u_{0}+a \tau$ with $|a|<\eta \cdot \min \left\{e^{\tau_{+}}, e^{\tau_{-}}\right\}, D_{u} \widehat{\partial} \oplus D \pi$ is surjective. Here $\tau_{ \pm}$are the asymptotic parameters of $u_{0}$.

Proof. In the previous section, we have proved the surjectivity of $D_{u_{0}} \bar{\partial}$, where $u_{0}$ is in $W_{\delta,(0 ; 2,0)}^{1, p}\left(\dot{\Sigma}, \mathbb{C}^{n}\right)$ satisfying $\bar{\partial} u_{0}=0$ with fixed asymptote $\gamma_{ \pm}$ and $\tau_{ \pm}$. Here the solution of $\bar{\partial} u=a$ is given by $u=u_{0}+a \tau$, so for small $a \in \mathbb{C}^{n} \backslash\{0\}, u=u_{0}+a \tau$ is a small perturbation from $u_{0}$ in $W_{\delta,(0 ; 2,0)}^{1, p}\left(\dot{\Sigma}, \mathbb{C}^{n}\right)$ by (7.1) and (7.2). Since surjectivity of $D_{u} \bar{\partial}$ is an open condition, which is preserved under small perturbation from $u$, for all $a$ with $|a|<\eta, D_{u} \bar{\partial}$ : $W_{\delta}^{1, p}\left(\dot{\Sigma}, u^{*} T \mathbb{C}^{n}\right) \rightarrow L_{\delta}^{p}\left(\dot{\Sigma}, \Lambda^{0,1}\left(u^{*} T \mathbb{C}^{n}\right)\right)$ is surjective, where $\eta$ is a constant depending on $u_{0}$.

Especially, this implies $D_{(u, a)} \widehat{\partial}$ is surjective since we can let $h=0$ in (7.3), and the target $L_{\delta}^{p}\left(\dot{\Sigma}, \Lambda^{0,1}\left(u^{*} T \mathbb{C}^{n}\right)\right)$, is still the same.

Next we describe the kernel of $\widehat{\partial}$ :

Proposition 7.3. For any $(u, a)$ satisfying $\widehat{\partial}(u, a)=0$,

$$
\begin{aligned}
\operatorname{ker}\left(D_{(u, a)} \widehat{\partial}\right) & =\left\{\left(U, V_{\mathbb{R}}^{ \pm}, V_{\mathcal{R}_{1}(\lambda)}^{ \pm}, h\right) \mid D_{u} \bar{\partial} U-h=0\right\} \\
& \cong\left\{\left(\operatorname{ker}\left(D_{u} \bar{\partial}\right)+h \tau, V_{\mathbb{R}}^{ \pm}, V_{\mathcal{R}_{1}(\lambda)}^{ \pm}, h\right)\right\} .
\end{aligned}
$$

Corollary 7.2. Index $D_{(u, a)} \widehat{\partial}=\operatorname{dim} \operatorname{ker} D_{(u, a)} \widehat{\partial}=6 n+3$

Proof. From (7.5), we see

$$
\operatorname{dim} \operatorname{ker} D_{(u, a)}=\operatorname{dim} \operatorname{ker} D_{u} \bar{\partial}+\operatorname{dim} T_{a} \mathbb{C}^{n}=4 n+3+2 n=6 n+3 .
$$

By Proposition 7.2, $D_{(u, a)} \widehat{\partial}$ is surjective so we get the index is equal to the dimension of the kernel.

On the moduli space $\mathcal{M}_{(0 ; 2,0)}^{+}\left(\dot{\Sigma}, \mathbb{C}^{n} ;(1,1)\right)$, using the isomorphism in Corollary 7.1 we define the jet evaluation map

$$
e v_{\text {jet }}: \mathcal{M}_{(0 ; 2,0)}^{+}\left(\dot{\Sigma}, \mathbb{C}^{n} ;(1,1)\right) \rightarrow \mathbb{C}^{n} \backslash\{0\},(u, a) \rightarrow a .
$$

$e v_{\text {jet }}$ has the following geometric meaning: For $u(\tau, t) \in \mathcal{M}_{(0 ; 2,0)}^{+}\left(\dot{\Sigma}, \mathbb{C}^{n}\right.$; $(1,1))$, let $I(\tau)=\int_{S^{1}} u(\tau, t) d t$ be the center of mass flow of $u(\tau, t)$, where the integration is with respect to the standard metric in $\mathbb{C}^{n}$, then $I^{\prime}(\tau)=$ $a=e v_{j e t}(u)$ for any $\tau$. (This is due to the mean value theorem of harmonic functions.) So $e v_{\text {jet }}(u)$ gives the direction that the local model $u$ is aligned to.

Definition 7.1 (Enhanced nodal Floer trajectory). We denote

$$
\mathcal{M}^{\text {nodal }}\left(\left[z_{-}, w_{-}\right],\left[z_{+}, w_{+}\right] ;(K, J),\left(f, J_{0}\right), p\right):=\left(e v_{\text {jet }}\right)^{-1}([\nabla f(p)])
$$


and define

$$
\begin{aligned}
& \mathcal{M}^{\text {nodal }}\left(\left[z_{-}, w_{-}\right],\left[z_{+}, w_{+}\right] ;(K, J),\left(f, J_{0}\right)\right) \\
& \quad=\bigcup_{p \in M} \mathcal{M}^{\text {nodal }}\left(\left[z_{-}, w_{-}\right],\left[z_{+}, w_{+}\right] ;(K, J),\left(f, J_{0}\right), p\right) .
\end{aligned}
$$

We call an element $\left(u_{+}, u_{0}, u_{+}\right)$therein an enhanced nodal Floer trajectory under the back ground Morse function $f$.

\section{Off-shell framework for the gluing}

We first define several function spaces to furnish the Banach manifolds and bundles needed for the $\bar{\partial}_{(K, J)}:=\left(d+P_{K}\right)_{J}^{(0,1)}$ operator to become a smooth Fredholm section of an appropriate infinite dimensional vector bundle. We summarize the various moduli spaces relevant to this formulation:

(1) For the moduli space $\mathcal{M}_{(0 ; 2,0)}^{\mathrm{SFT}}\left(\mathbb{C}^{n}\right)$, this is the Morse-Bott setting of the Symplectic Field Theory. We have followed the description by Fukaya-Oh-Ohta-Ono [FOOO2] of the Fredholm theory where a similar Morse-Bott setting of SFT but with Lagrangian boundary condition was used. There was also given a Morse-Bott setup of the Fredholm theory of SFT by Bourgeois [Bou].

(2) For the moduli space $\mathcal{M}_{(1 ; 1,0)}\left((K, J) ; z_{-}\right)$or $\mathcal{M}_{(1 ; 0,1)}\left((K, J) ; z_{+}\right)$, this is standard except the requirement that the maps are immersed at the origin.

(3) For the moduli space of nodal Floer trajectories, it is necessary to match the evaluation maps from (1) and (2). We will introduce a cylindrical metric on a neighborhood of the nodal point $p=u_{-}\left(o_{-}\right)=u_{+}\left(o_{+}\right)$in $M$ for any element $u=\left(u_{-}, u_{+}\right)$with $u_{-} \in \mathcal{M}\left((K, J) ;\left[z_{-}, w_{-}\right]\right)$and $u_{+} \in \mathcal{M}\left((K, J) ;\left[z_{+}, w_{+}\right]\right)$, such that the evaluation map of (2) takes the value in SFT setting. Geometrically this corresponds to blowing up of the neighborhood of $p$ and then reparameterization by $S^{2 n-1} \times \mathbb{R}$. This setting is very similar to that in [FOOO2] in Lagrangian surgery on a vertex of a holomorphic triangle. However, instead of blowing up a given vertex in $\mathrm{M}$, here we need to do this reparametrization for a family of neighborhoods depending on varying $p$, so we need a family of cylindrical metrics fibered over $M$. The precise off-shell formulation is in the subsequent subsection.

(4) For the moduli space of "disk-flow-disk", we need to formulate a Fredholm theory for the objects which are allowed to have dimension jump. When the length of the lines of "disk-flow-disk" elements shrink to zero, there appears some subtlety in the Fredholm theory since we encounter a noncompact family of domains and suitable transversality 
is needed for such a family. However, this transversality issue can be reduced to a finite dimensional differential topology lemma and is solved in Section 9.

To apply the discussion on $\mathbb{C}^{n}$ to the one-jet consideration of $M$, we need a way of identifying $\left(T_{x} M, \omega_{x}, J_{x}\right)$ with the standard almost Kähler structure on $\left(\mathbb{C}^{n}, \omega_{0}, J_{0}\right)$. Since this identification depends on the point $x$, there is no canonical way of doing this identification, especially when $J$ is nonintegrable. One might try to adjust $J$ so that it becomes integrable if the given point $x$ were a fixed point. However for our purpose, we will need to provide this identification at an unspecified point and so changing the given almost complex structure is not appropriate. Because of this, we need to carry out this identification in a systematic way making all the choices involved smoothly varying over $x \in M$.

It turns out the notion of Darboux family introduced by Weinstein [We1] is particularly useful for the above process.

8.1. Darboux family and explosion of manifolds. In this section, we first recall the notion of Darboux family introduced by Weinstein [We1] and then carry out the explosion of manifolds of the Riemannian metric at a point to produce a smooth family parameterized by $M \times\left[0, \varepsilon_{0}\right]$ for a constant $\varepsilon_{0}$ depending only on the symplectic manifold $(M, \omega)$.

For a symplectic manifold $M$, each tangent space $T_{x} M$ inherits the structure of symplectic vector space with the symplectic quadratic form $\omega_{x}$.

Definition 8.1. [We1] A Darboux family is a family of symplectic diffeomorphisms $I_{x}: V_{x} \rightarrow U_{x}$ such that

(1) $V_{x}$ and $U_{x}$ are open neighborhoods of $0 \in T_{x} M$ and $x \in M$, respectively,

(2) $I_{x}(0)=x$ and $d I_{x}(0)=\mathrm{i} d$,

(3) $I_{x}^{*} \omega=\omega_{x}$,

(4) $\left(V_{x}, U_{x} ; I_{x}\right)$ depends smoothly on $x$.

To emphasize the readers that $I_{x}$ plays the same kind of role as the exponential map at $x$, we denote

$$
I_{x}=: \exp _{x}^{I} .
$$

When $(M, \omega)$ is equipped with a compatible almost complex structure $J$ so that the triple $(M, \omega, J)$ defines an almost Kähler structure, the above Darboux family automatically assigns an almost complex structure $I_{x}^{*} J$ on $V_{x}$. In addition to (1), we can require the condition

(5) $\left(I_{x}^{*} J\right)(0)=J_{x}$ on $T_{0}\left(T_{x} M\right) \cong T_{x} M$.

Now we can identify $\left(T_{x} M, \omega_{x}, J_{x}\right)$ with $\mathbb{C}^{n}$ by an Hermitian isometry and denote by $B^{2 n}\left(\varepsilon_{0} ; J_{x}\right)$ as the standard ball of radius $\varepsilon_{0}>0$. The ball 
$B^{2 n}\left(\varepsilon_{0} ; J_{x}\right) \subset T_{x} M$ does not depend on this identification but depends on $\left(\omega_{x}, J_{x} ; \varepsilon_{0}\right)$.

Note that when $M$ is compact, we can choose the family so that there is $\varepsilon_{0}>0$ such that

$$
I_{x}\left(B^{2 n}\left(\varepsilon_{0} ; J_{x}\right)\right) \subset U_{x}
$$

for all $x \in M$. We call any such $\varepsilon_{0}>0$ an admissible radius for the Darboux family. We denote

$$
B\left(I, J ; x, \varepsilon_{0}\right)=I_{x}\left(B^{2 n}\left(\varepsilon_{0} ; J_{x}\right)\right) \subset M .
$$

Since we will not change $J$ or $I$, we will simplify and just denote $B\left(I, J ; x, \varepsilon_{0}\right)$ by $B_{\varepsilon_{0}}(x)$ whenever there is no danger of confusion.

Next we recall the explosion constructions of manifolds and metrics from [We2] in detail in Appendix 8.4. We use this construction in the context of almost Kähler structure.

Consider the pointed manifold $(M, p)$ for each $p \in M$ and denote by $\pi_{E(M, p)}: E(M, p) \rightarrow M \times \mathbb{R}$ the explosion of $(M, p)$ at $p$. By construction, $E(M, p)$ is defined by beginning with the product $M \times \mathbb{R}$, removing the "axis" $M \times\{0\}$, and replacing it with the tangent space $T_{p} M$ at $p$ in $M$. The differentiable structure on $E(M, p)$ is taken to be the usual product structure on $M \times(\mathbb{R} \backslash\{0\})$. Charts near $M \times\{0\}$ is defined with the aid of the above given Darboux family of coordinates on $M$. We refer to [We2] or Appendix 8.4 for more precise details. This enables us to regard $E(M, p)$ as a family of manifolds "exploding" at $p$ at the time $\varepsilon=0$. For $\varepsilon \neq 0$, the fiber $E_{\varepsilon}=E(M, p)_{\varepsilon}$ is diffeomorphic to $M$ and for $\varepsilon=0, E_{0}$ is diffeomorphic to the linear space $T_{p} M$.

We now consider the explosions $E(M, p)$ as a family parameterized by $p \in M$. We define

$$
E(M)=\bigcup_{p \in M} E(M, p) \rightarrow M
$$

and will provide a fiber bundle structure $E(M) \rightarrow M$ : It is enough to provide compatible local trivializations thereof at each $p \in M$. Let $U \subset M$ be a neighborhood of $p$ such that $I^{-1}(U)=B^{2 n}(r)$. Without loss of any generality, we will assume $r=1$. We will find a trivialization

$$
\Phi:\left.E(M)\right|_{U} \cong U \times E(M, p)
$$

by defining diffeomorphisms $\varphi_{p^{\prime} p}: E\left(M, p^{\prime}\right) \rightarrow E(M, p)$ depending smoothly on $p^{\prime} \in U$. For this purpose, we will use Corollary 8.2 in the Appendix.

Using the fact that the open ball $\operatorname{Int} B^{2 n}(1)$ is two-point homogeneous under the action of Möbius transformations we can find a smooth family of diffeomorphism

$$
\varphi_{p^{\prime} p}:\left(B^{2 n}(1), \partial B^{2 n}(1)\right) \rightarrow\left(B^{2 n}(1), \partial B^{2 n}(1)\right),
$$




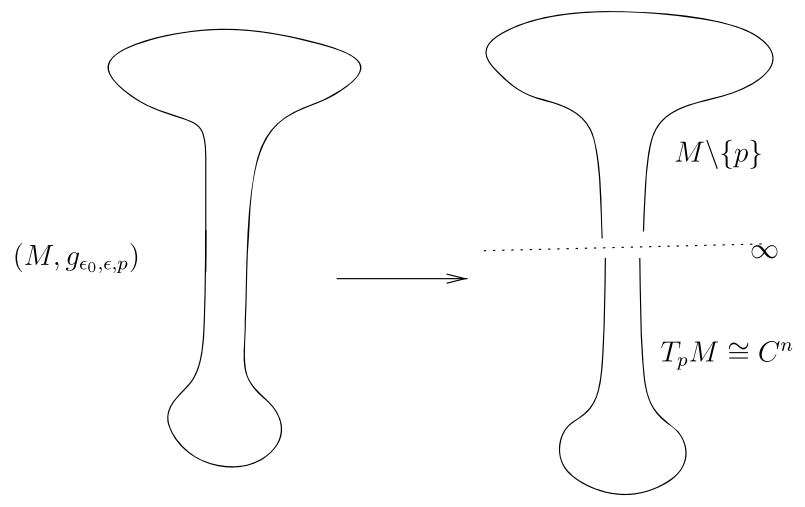

Figure 4. Explosion of a manifold at $p$.

which maps $p^{\prime} \rightarrow p$ and is the identity on $\left.\partial B^{2 n}(1)\right)$. Restricting $p^{\prime} \in B^{2 n}(1-$ $\kappa$ ) for a fixed small $\kappa>0$, and suitably modifying the diffeomorphism on $\operatorname{Int}\left(B^{2 n}(1)\right) \backslash B^{2 n}(1-\kappa)$ once and for all, we can smoothly extend outside of $B^{2 n}(1)$ by setting it to be the identity. This defines a diffeomorphism $\varphi_{p^{\prime} p}^{I}:\left(M,\left\{p^{\prime}\right\}\right) \rightarrow(M,\{p\})$ which is the identity outside $U$. Furthermore one can easily arrange that as $p^{\prime} \rightarrow p$, the map $\varphi_{p p^{\prime}} \rightarrow i d$ in $C^{\infty}$-topology.

By making the above modification on $\operatorname{Int}\left(B^{2 n}(1)\right) \backslash B^{2 n}(1-\kappa)$ once and for all, the local trivializations over different $U$ will be compatible and hence we have shown that $E(M) \rightarrow M \times \mathbb{R}$ is locally trivial.

One can see this explosion process more vividly if we consider it in the point of view of Riemannian manifolds (Figure 4). Let $g$ be a given metric on $M$ and $0<\operatorname{inj}(g)<\infty$ be the injective radius of $g$. Fix a constant $\varepsilon_{0}$ that $0<\varepsilon_{0}<\operatorname{inj}(g)$. In our case, we will consider the compatible metric $g=\omega(\cdot, J \cdot)$. We will introduce a family of Riemannian metrics on $M$ for $\varepsilon>0$, denoted by $g_{\varepsilon_{0}, \varepsilon, p}$, in a way that the family satisfies the following properties:

Proposition 8.1. There exists a family of Riemannian metrics $g_{\delta, p}$ on $M$ for $\delta>0$ such that

(1) $g_{\delta, p} \equiv g$ for $\delta \geq 2 \varepsilon_{0}$,

(2) The fiberwise pull-back $\left.\pi_{E(M, p)}^{*}\left(g_{\delta, p}\right)\right|_{E_{\delta}}$ over $M \times \mathbb{R}_{+} \backslash\{0\}$ extends smoothly to $M \times \mathbb{R}_{+}$by defining the metric $g_{0, p}$ on $E_{0} \cong T_{p} M$ to be $g_{0, p}=g(p)$.

Proof. In regard to the expression (8.21) of the coordinate chart applied to $Y=\{p\}$

$$
E(I)(x, \varepsilon)=(I(\varepsilon x), \varepsilon)
$$


we want the family $g_{\delta, p}$ to be defined by

$$
g_{\delta, p}=\frac{1}{\delta^{2}} I^{*} g
$$

for $\delta$ near 0 . Then the pull-back metric of $g_{\delta, p}$ to $E_{\delta}$ is given by

$$
\left.\pi_{E(M, p)}^{*} g_{\delta, p}(x, \delta)\right|_{T E_{\delta}}=\frac{1}{\delta^{2}}\left(E(I) \circ R_{\delta}\right)^{*} g(x, \delta)
$$

in the coordinate chart $E(I)$ on $E(U)$. Now a straightforward calculation shows that this family has the coordinate expression as

$$
\pi_{E(M, p)}^{*} g_{\delta, p}(x, \delta)(v)=g\left(I \circ R_{\delta}(x)\right)\left(T_{R_{\delta}(x)} I(v)\right)
$$

for $v \in T E_{\delta}$ at $(x, \delta)$ with respect to the canonical coordinate $E(I)$ associated to the Darboux chart $I$ at $p$. From this it follows that this family smoothly extends to $E(M, p)$ across $\delta=0$, if we set the metric $g_{0, p}$ to be $g(p)$ : Here we use the condition $T_{0} I=\mathrm{i} d$ on $T_{p} M$.

For any given $0<\varepsilon \leq \varepsilon_{0}$, we can interpolate the scaled metric $g / \varepsilon_{0}^{2}$ and $g / \varepsilon^{2}$ on $B_{\varepsilon_{0}}(p)$ via cylindrical metric. More precise description of the metrics is in order.

Using the Darboux family, for any $p \in M$, we define a one-parameter family of metrics $g_{\varepsilon_{0}, \varepsilon, p}$ on $M$ as the following:

$$
g_{\varepsilon_{0}, \varepsilon, p}= \begin{cases}\frac{1}{\varepsilon_{0}^{2}} g, & x \in M \backslash B_{\varepsilon_{0}}(p), \\ \rho_{+}(x) \frac{1}{\varepsilon_{0}^{2}} g+\left(1-\rho_{+}-\rho_{-}\right) \frac{I_{*}(g(p))}{\left(r \circ\left(\exp _{p}^{I}\right)^{-1}\right)^{2}} & \\ \quad+\rho_{-}(x) \frac{1}{\varepsilon^{2}} I_{*}(g(p)), & x \in B_{\varepsilon_{0}}(p) \backslash B_{\varepsilon}(p), \\ \frac{1}{\varepsilon^{2}} I_{*}(g(p)), & x \in B_{\varepsilon}(p),\end{cases}
$$

where the $r$ is the radius function on $\left(T_{p} M, g(p)\right)$, the $\rho_{+}(x)$ is a smooth cut function that $\rho_{+}(x)=1$ outside $B_{\varepsilon_{0}}(p)$ and $\rho(x)=0$ in $B_{\frac{9}{10} \varepsilon_{0}}(p)$, while $\rho_{-}(x)$ is another smooth cut function that $\rho_{-}(x)=0$ outside $B_{\frac{11}{10} \varepsilon}(p)$ and $\rho(x)=1$ in $B_{\varepsilon}(p)$.

We equip $M \backslash\{p\}$ with a metric $g_{\varepsilon_{0}, p}$ making $M \backslash\{p\}$ a manifold with one cylindrical end, where

$$
g_{\varepsilon_{0}, p}= \begin{cases}\frac{1}{\varepsilon_{0}^{2}} g, & x \in M \backslash B_{\varepsilon_{0}}(p), \\ \rho_{+}(x) \frac{1}{\varepsilon_{0}^{2}} g+\left(1-\rho_{+}(x)\right) \frac{I_{*}(g(p))}{\left(r \circ\left(\exp _{p}^{I}\right)^{2}-1\right)^{2}}, & x \in B_{\varepsilon_{0}}(p) .\end{cases}
$$

We also equip $T_{p} M$ with a metric $g_{\mathrm{cyl}, \varepsilon, p}$ making it to be a manifold with one cylindrical end as well, where

$$
g_{\mathrm{cyl}, \varepsilon, p}= \begin{cases}\left(1-\rho_{-}(x)\right) \frac{g(p)}{r^{2}}+\rho_{-}(x) \frac{1}{\varepsilon^{2}} g(p), & x \in T_{p} M \backslash B_{\varepsilon}(p), \\ \frac{1}{\varepsilon^{2}} g(p), & x \in B_{\varepsilon}(p) .\end{cases}
$$


Clearly, the scaling map $\varepsilon:\left(T_{p} M, g_{\mathrm{cyl}, 1, p}\right) \rightarrow\left(T_{p} M, g_{\mathrm{cyl}, \varepsilon, p}\right), v \mapsto v / \varepsilon$ is an isometry and $\left(T_{p} M, g_{\mathrm{cyl}, 1, p}\right)$ is isometric to $\left(\mathbb{C}^{n}, g_{\mathrm{cyl}, 1,0}\right)$.

Remark 8.1. We have the following observations:

(1) The metric $g_{\varepsilon_{0}, \varepsilon, p}$ on $M$ is the interpolation of the metrics $g_{\varepsilon_{0}, p}$ and $g_{\mathrm{cyl}, \varepsilon, p}$.

(2) $\lim _{\varepsilon \rightarrow 0} g_{\varepsilon_{0}, \varepsilon, p}=g_{\varepsilon_{0}, p}$ on $M \backslash\{p\}$.

(3) The expression $\frac{I_{*}(g(p))}{\left(r \circ\left(\exp _{p}^{I}\right)^{-1}\right)^{2}}$ is simply the push-forward of the cylindrical metric $\frac{g}{r^{2}}=g_{\mathbb{R} \times S^{2 n-1}}$ on $T_{p} M \backslash\{p\}$ to $M$ by $\exp _{p}^{I}$.

(4) The degenerating metric $g_{\varepsilon_{0}, \varepsilon, p}$ on $M$ given is noncollapsing as $\varepsilon \rightarrow 0$, and

$$
g_{\varepsilon_{0}, p}=\lim _{\varepsilon \rightarrow 0} g_{\varepsilon_{0}, \varepsilon, p}, \quad \text { on } M \backslash\{p\} .
$$

in the Gromov-Hausdorff topology.

We note that $B_{\varepsilon_{0}}(p) \backslash B_{\varepsilon}(p)$ is identified with

$$
\left(0, \ln \left(\varepsilon_{0}\right)-\ln \varepsilon\right] \times S^{2 n-1} \subset \mathbb{R} \times S^{2 n-1}
$$

via the map $(r, \Theta) \mapsto(s, \Theta)$ with $s=\ln r$. In the coordinates $(s, \Theta)$, any point $x \in B_{\varepsilon_{0}}(p)$ is identified to a pair

$$
(s(x), \Theta(x)) \in(-\infty, 0] \times S^{2 n-1} .
$$

We call $(s, \Theta)$ the cylindrical coordinate chart near $p$.

Now we equip $E(M) \times M \times \mathbb{R}_{+} \rightarrow M \times \mathbb{R}_{+}$with the structure of Riemannian fibration with its fibers given by

$$
\begin{cases}\left(M, g_{\varepsilon_{0}, \varepsilon, p}\right), & \text { for }(p, \varepsilon) \in M \times \mathbb{R}_{+}, \varepsilon \neq 0 \\ \left(T_{p} M, g(p)\right), & \text { for }(p, \varepsilon)=(p, 0) .\end{cases}
$$

This fibration over $\varepsilon>0$ will host the off-shell Banach manifolds for the resolved Floer trajectories arising from nodal Floer trajectories, while the union

$$
E_{0} \#(M \backslash\{p\})=T_{p} M \#(M \backslash\{p\})
$$

regarded as the end-connected sum of two symplectic manifolds $E_{0}$ and $M \backslash\{p\}$ : Here $E_{0}$ has convex end and $M \backslash\{p\}$ has concave end both with the unit sphere $S^{1}\left(T_{p} M\right)$ as their asymptotic boundaries in the Darboux chart $I_{p}$. This explosion $E(M) \times M \times \mathbb{R}_{+} \rightarrow M \times \mathbb{R}_{+}$will be implicitly used to define a Banach manifold that host the enhanced nodal Floer trajectories. We turn to the description of these off-shell Banach manifolds in the next couple of subsections. 
8.2. Off-shell formulation for perturbed $J$-holomorphic discs. The off-shell formulation of the moduli space $\mathcal{M}_{(0 ; 2,0)}^{\mathrm{SFT}}\left(\mathbb{C}^{n}\right)$ has been given in Subsection 5.2. Here we provide the off-shell setting for the perturbed $\boldsymbol{J}$-holomorphic disk moduli spaces $\mathcal{M}\left(\left(K^{-}, J^{-}\right) ;\left[z_{-}, w_{-}\right] ; A_{-}\right)$and $\mathcal{M}\left(\left(K^{+}, J^{+}\right) ;\left[z_{+}, w_{+}\right] ; A_{+}\right)$. Note that if the target is the compact manifold $M$, then the off-shell Banach manifold hosting perturbed $\boldsymbol{J}$-holomorphic curves (or discs) is the standard $W^{1, p}(\dot{\Sigma}, M)$. That formulation was used in "disk-flow-disk" sections.

However, to resolve enhanced nodal Floer trajectories by the scaled gluing method, we need to blow up every point $p$ in $M$. Therefore, the off-shell Banach manifold should incorporate this fact for which we need to consider a family of Banach manifold, which forms a (locally trivial) fiber bundle over the target manifold $M$.

We first introduce the Banach manifold $W_{\alpha}^{1, p}\left(\dot{\Sigma}, M ; p, z_{+}, \gamma_{+}, \tau_{+}\right)$for each given $\left(p, z_{+}, \gamma_{+}, \tau_{+}\right)$. Here $\alpha=\alpha(\tau)$ stands for the weighting function that

$$
\alpha(\tau)= \begin{cases}\mathrm{e}^{2 \pi \delta|\tau|}, & \tau \leq 0, \\ 1, & \tau \geq 0 .\end{cases}
$$

The space $W_{\alpha}^{1, p}\left(\dot{\Sigma}, M ; p, z_{+}, \gamma_{+}, \tau_{+}\right)$consists of elements $u:\left(\dot{\Sigma}, o_{+}\right) \rightarrow$ $(M, p)$ satisfying the following:

(1) $u \in W_{l o c}^{1, p}$

(2) In the analytical chart of a positive puncture $e_{+} \in \Sigma, \lim _{\tau \rightarrow+\infty}$ $u(\tau, t)=z_{+}(t)$ for the periodic orbit $z_{+}(t)$ in $M$.

(3) For sufficiently large $\tau, u(\tau, t)=\exp _{z_{+}(t)}(\xi(\tau, t))$, and $\xi(\tau, t) \in$ $L^{p}\left([0,+\infty) \times S^{1}, z_{+}^{*}(T M)\right)$.

(4) In the analytical chart $D_{+} \cong(-\infty, 0] \times S^{1}$ of the marked point $o_{+}$, $u(\tau, t)$ is in a cylindrical coordinate chart $B_{\varepsilon_{0}}(p)$ of $p$, with $u(\tau, t)=$ $\left(\Theta_{+}(\tau, t), s_{+}(\tau, t)\right)$, satisfying

$$
\begin{aligned}
& \mathrm{e}^{\frac{2 \pi \delta|\tau|}{p}}\left\|\Theta_{+}(\tau, t)-\gamma_{+}(t)\right\|_{S^{2 n-1}} \in W^{1, p}\left((-\infty, 0] \times S^{1}, \mathbb{R}\right), \\
& \mathrm{e}^{\frac{2 \pi \delta|\tau|}{p}}\left|s_{+}(\tau, t)-2 \pi\left(\tau-\tau_{+}\right)\right| \in W^{1, p}\left((-\infty, 0] \times S^{1}, \mathbb{R}\right)
\end{aligned}
$$

for the simple Reeb orbit $\gamma_{+}(t)$ and $\tau_{+} \in \mathbb{R}$.

$W_{\alpha}^{1, p}\left(\dot{\Sigma}, M ; p_{-}, z_{-}, \gamma_{-}, \tau_{-}\right)$is defined similarly, but the weight function $\alpha$ is replaced by $\alpha(-\tau)$.

Remark 8.2. We only consider simple Reeb orbits because we have chosen a generic $J$ so that the nodal point $p=u_{-}\left(o_{-}\right)=u_{+}\left(o_{+}\right)$of any nodal Floer trajectory $\left(u_{-}, u_{+}\right)$is immersed. Translating this to the asymptote of $u_{ \pm}$in the cylindrical manifold $M \backslash\{p\}$, we only get simple Reeb orbits in $S^{2 n-1}$. Therefore, to host such $u_{ \pm}$with immersed at $o_{ \pm}$, the function spaces $W_{\alpha}^{1, p}\left(\dot{\Sigma}, M ; p_{ \pm}, z_{ \pm}, \gamma_{ \pm}, \tau_{ \pm}\right)$with simple Reeb orbits $\gamma_{ \pm}$are adequate. 
We denote the set of parameterized simple Reeb orbits $\gamma(t)$ in $S^{2 n-1}$ by $\widetilde{\mathcal{R}}_{1}(\lambda)$. By the Hopf fibration $S^{2 n-1} \rightarrow \mathbb{C} P^{n}$ we see $\widetilde{\mathcal{R}}_{1}(\lambda) \cong S^{2 n-1}$, because given any point in $S^{2 n-1}$ to start, the passing $S^{1}$ fiber is a simple Reeb orbit.

Remark 8.3. Since only finitely many nodal Floer trajectories $\left(u_{-}, u_{+}\right)$ are involved during gluing, we can assume $\left\|\nabla u_{ \pm}\left(o_{ \pm}\right)\right\|$(the gradient is with respect to the metric $g$ on $M$ ) is uniformly bounded. Therefore by possibly shrinking the cylindrical charts $O_{ \pm}$, we can assume they all satisfy $u_{ \pm}\left(O_{ \pm}\right) \subset$ $U_{p}$. Therefore, for $(\tau, t) \in O_{ \pm}, u_{ \pm}(\tau, t)$ is in the cylindrical coordinate of the target $\left(M \backslash\{p\}, g_{\delta, p}\right)$. This said, in cylindrical coordinates of the domain and target, the map

$$
u_{ \pm}: O_{ \pm} \rightarrow B_{\varepsilon_{0}}(p) \cong(-\infty, 0] \times S^{2 n-1},(\tau, t) \rightarrow\left(s_{ \pm}(\tau, t), \Theta_{ \pm}(\tau, t)\right)
$$

has the asymptote satisfying

$$
\begin{aligned}
& \left|\nabla^{k}\left(\Theta_{ \pm}(\tau, t)-\gamma_{ \pm}(t)\right)\right|_{S^{2 n-1}} \leq C_{k} \mathrm{e}^{\frac{-2 \pi c_{k}|\tau|}{p}} \quad \text { and } \\
& \left|\nabla^{k}\left(s \pm(\tau, t)-2 \pi\left(\tau-\tau_{ \pm}\right)\right)\right| \leq C_{k} \mathrm{e}^{\frac{-2 \pi c_{k}|\tau|}{p}}
\end{aligned}
$$

for some constant $C_{k}$ and $c_{k}$, where $s_{ \pm}=s \circ u_{ \pm}$and $\Theta_{ \pm}=\Theta \circ u_{ \pm}$. The $C_{k}$ and $c_{k}$ can be made uniform for all $u_{ \pm}$nearby the (finitely many) nodal Floer trajectories $\left(u_{-}, u_{+}\right)$by the continuity of the $\nabla^{k+1} u_{ \pm}$translated into cylindrical coordinate. We chose $\delta$ in the definition of $W_{\alpha}^{1, p}\left(\dot{\Sigma}, M ; p_{ \pm}, z_{ \pm}, \gamma_{ \pm}, \tau_{ \pm}\right)$ to be less than $c_{k}(k=0,1,2)$.

Then we let

$$
W_{\alpha}^{1, p}\left(\dot{\Sigma}, M ; z_{+}\right):=\bigcup_{p \in M} \bigcup_{\left(\gamma_{+}, \tau_{+}\right) \in \widetilde{\mathcal{R}}_{1}(\lambda) \times \mathbb{R}} W_{\alpha}^{1, p}\left(\dot{\Sigma}, M, p, z_{+}, \gamma_{+}, \tau_{+}\right),
$$

where $\widetilde{\mathcal{R}}_{1}(\lambda)$ is the set of all parameterized simple Reeb orbits in $S^{2 n-1}$. So $W_{\alpha}^{1, p}\left(\dot{\Sigma}, M ; z_{+}\right)$is the space that hosts all $\left(K_{-}, J_{-}\right)$-holomorphic discs $u_{+}$ with boundary on the periodic orbit $z_{+}(t)$ in $M$, and immersion at $o_{+}$. The moduli space $W_{\alpha}^{1, p}\left(\dot{\Sigma}, M ; z_{-}\right)$is defined similarly.

We will show $W_{\alpha}^{1, p}\left(\dot{\Sigma}, M ; z_{+}\right)$is a Banach manifold. First, we describe the tangent space of a given element $u$ in $W_{\alpha}^{1, p}\left(\dot{\Sigma}, M ; z_{+}\right)$. Since

$$
\pi: W_{\alpha}^{1, p}\left(\dot{\Sigma}, M ; z_{+}\right) \rightarrow M
$$

is a fiber bundle with its fiber at $p \in M$ given by

$$
W_{\alpha}^{1, p}\left(\dot{\Sigma}, M, p, z_{+}\right):=\bigcup_{\left(\gamma_{+}, \tau_{+}\right) \in \widetilde{\mathcal{R}}_{1}(\lambda) \times \mathbb{R}} W_{\alpha}^{1, p}\left(\dot{\Sigma}, M, p, z_{+}, \gamma_{+}, \tau_{+}\right)
$$

we need to consider both the vertical and horizontal variations for $u$. 
Let $\chi_{+}:(-\infty, 0] \rightarrow[0,1]$ be a smooth function such that $\chi_{+}(\tau)=1$ for $\tau \leq-2$ and $\chi_{+}(\tau)=0$ for $\tau \geq-1$. We consider the quadruple $\left(U, V_{\widetilde{\mathcal{R}}_{1}(\lambda)}^{+}\right.$, $\left.V_{\mathbb{R}}^{+}, v_{+}\right)$satisfying

(1) $V_{\widetilde{\mathcal{R}}_{1}(\lambda)}^{+} \in T_{\gamma_{+}} \widetilde{\mathcal{R}}_{1}(\lambda), V_{\mathbb{R}}^{+} \in \mathbb{R}=T_{\tau_{+}} \mathbb{R}$, and $v_{+} \in T_{p} M$, where $p=u\left(o_{+}\right) ;$

(2) $U \in W_{\text {loc }}^{1, p}\left(\left(\dot{\Sigma}, u^{*} T M\right)\right.$;

(3) $U \in W_{\delta}^{1, p}\left([0,+\infty) \times S^{1}, u^{*} T M\right)$, where $[0,+\infty) \times S^{1}$ is the analytical chart for the positive puncture $e_{+} \in \Sigma$;

(4) In the analytical chart $D_{+} \cong(-\infty, 0] \times S^{1}$ of the marked point $o_{+}$, $u(\tau, t)$ is in the cylindrical chart of $p \in M$. Let

$$
\widetilde{U}(\tau, t)=U(\tau, t)-\chi_{+}(\tau) \operatorname{Pal}_{u(\tau, t)} U(-\infty, t),
$$

then $\mathrm{e}^{\frac{2 \pi \delta|\tau|}{p}}|\widetilde{U}(\tau, t)| \in W^{1, p}\left((-\infty, 0) \times S^{1}, \mathbb{R}\right)$. Here $U(-\infty, t)=V_{\widetilde{\mathcal{R}}_{1}(\lambda)}^{+}$ $(t)$, and $\operatorname{Pal}_{u(\tau, t)} U(-\infty, t)$ is the parallel transport of $U(-\infty, t)$ from $u(-\infty, t)$ to $u(\tau, t)$ along the minimal geodesic in $\left(M, g_{\varepsilon_{0}, p}\right)$.

Let $C^{0}(u)$ be the set of all such quadruples. It becomes a Banach space with the norm

$$
\left\|\left(U, V_{\widetilde{\mathcal{R}}_{1}(\lambda)}^{+}, V_{\mathbb{R}}^{+}, v_{+}\right)\right\|_{1, p, \alpha}^{p}=\left\|\mathrm{e}^{\frac{2 \pi \delta|\tau|}{p}} \widetilde{U}(\tau, t)\right\|_{W^{1, p}}^{p}+\left|V_{\mathbb{R}}^{+}\right|^{p}+\left|V_{\widetilde{\mathcal{R}}_{1}(\lambda)}^{+}\right|^{p}+\left|v_{+}\right|^{p} .
$$

Then it is standard to check $W_{\alpha}^{1, p}\left(\dot{\Sigma}, M ; z_{+}\right)$is a Banach manifold and

$$
C^{0}(u)=T_{u} W_{\alpha}^{1, p}\left(\dot{\Sigma}, M ; z_{+}\right) .
$$

Similarly, $W_{\alpha}^{1, p}\left(\dot{\Sigma}, M ; z_{-}\right)$is a Banach manifold. The tangent vector

$$
\left(U, V_{\widetilde{\mathcal{R}}_{1}(\lambda)}^{-}, V_{\mathbb{R}}^{-}, v_{-}\right) \in T_{u} W_{\alpha}^{1, p}\left(\dot{\Sigma}, M ; z_{-}\right)
$$

is defined similarly, where $V_{\widetilde{\mathcal{R}}_{1}(\lambda)}^{-} \in T_{\gamma_{-}} \widetilde{\mathcal{R}}_{1}(\lambda), V_{\mathbb{R}}^{-} \in \mathbb{R}=T_{\tau_{-}} \mathbb{R}$, and $v_{-} \in$ $T_{p} M, p=u\left(o_{-}\right)$. The Banach norm is

$$
\left\|\left(U, V_{\widetilde{\mathcal{R}}_{1}(\lambda)}^{-}, V_{\mathbb{R}}^{-}, v_{-}\right)\right\|_{1, p, \alpha}^{p}=\left\|\mathrm{e}^{\frac{2 \pi \delta|\tau|}{p}} \widetilde{U}(\tau, t)\right\|_{W^{1, p}}^{p}-\left|V_{\mathbb{R}}^{+}\right|^{p}+\left|V_{\widetilde{\mathcal{R}}_{1}(\lambda)}^{-}\right|^{p}+\left|v_{-}\right|^{p} .
$$

Remark 8.4. $U$ corresponds to the variation of $u$ within a fixed fiber $M \backslash\{p\}$, and $v_{+} \in T_{p} M$ corresponds to the variation of the fiber $M \backslash\{p\}$ in $\widetilde{M}$.

$$
\begin{aligned}
& \text { Let } \\
& \qquad \begin{array}{r}
\mathcal{B}_{+}=W_{\alpha}^{1, p}\left(\dot{\Sigma}, M ; z_{+}\right), \quad \mathcal{L}_{+}=\bigcup_{u \in \mathcal{B}_{+}} L_{\alpha}^{p}\left(\dot{\Sigma}, \Lambda_{J^{+}}^{0,1}\left(u_{+}^{*}(T M)\right)\right), \quad \text { and } \\
\bar{\partial}_{\left(J^{+}, K^{+}\right)}: \mathcal{B}_{+} \rightarrow \mathcal{L}_{+}, \quad(u, p) \rightarrow\left(\bar{\partial}_{J^{+}} u+\left(P_{K^{+}}\right)_{J^{+}}^{(0,1)}(u), p\right) .
\end{array}
\end{aligned}
$$


Then $\bar{\partial}_{\left(J^{+}, K^{+}\right)}$is a section of the Banach bundle $\mathcal{B}_{+} \rightarrow \mathcal{L}_{+}$, and the perturbed $\left(J^{+}, K^{+}\right)$-holomorphic disk moduli space $\mathcal{M}\left(J^{+}, K^{+} ; z_{+}\right)$can be written as the zero set

$$
\mathcal{M}_{1}\left(J^{+}, K^{+} ; z_{+}\right)=\left(\bar{\partial}_{\left(J^{+}, K^{+}\right)}\right)^{-1}(0) .
$$

When we consider the moduli space with more topological restrictions on $u$, say $\mathcal{M}_{1}\left(K^{+}, J^{+} ;\left[z_{+}, w_{+}\right] ; A_{+}\right)$, we can accordingly restrict to

$$
\bar{\partial}_{\left(J^{+}, K^{+}\right)}: \mathcal{B}_{+}\left(\left[z_{+}, w_{+}\right] ; A_{+}\right) \rightarrow \mathcal{L}_{+}\left(\left[z_{+}, w_{+}\right] ; A_{+}\right),
$$

and get $\mathcal{M}_{1}\left(K^{+}, J^{+} ;\left[z_{+}, w_{+}\right] ; A_{+}\right)=\left(\bar{\partial}_{\left(J^{+}, K^{+}\right)}\right)^{-1}(0)$. Here

$$
\mathcal{B}_{+}\left(\left[z_{+}, w_{+}\right] ; A_{+}\right)=\left\{u \in \mathcal{B}_{+} \mid\left[u \# w_{+}\right]=A_{+}\right\}
$$

and so $\mathcal{B}_{+}=\mathcal{B}_{+}\left(z_{+}\right)$is decomposed into

$$
\mathcal{B}_{+}=\bigcup_{A_{+}} \mathcal{B}_{+}\left(\left[z_{+}, w_{+}\right] ; A_{+}\right) .
$$

And

$$
\mathcal{L}_{+}\left(\left[z_{+}, w_{+}\right] ; A_{+}\right)=\left.\mathcal{L}_{+}\right|_{\mathcal{B}_{+}\left(\left[z_{+}, w_{+}\right] ; A_{+}\right)} \rightarrow \mathcal{B}_{+}\left(\left[z_{+}, w_{+}\right] ; A_{+}\right)
$$

is the restriction of the bundle $\mathcal{L}_{+} \rightarrow \mathcal{B}_{+}$to $\mathcal{B}_{+}\left(\left[z_{+}, w_{+}\right] ; A_{+}\right)$. We also note that $\mathcal{B}_{+}\left(\left[z_{+}, w_{+}\right] ; A_{+}\right)$has the decomposition

$$
\mathcal{B}_{+}\left(\left[z_{+}, w_{+}\right] ; A_{+}\right)=\bigcup_{p \in M} \mathcal{B}_{+}\left(\left[z_{+}, w_{+}\right], p ; A_{+}\right)
$$

that is a fiber bundle over $M$ with its fiber at $p \in M$ given by

$$
\mathcal{B}_{+}\left(\left[z_{+}, w_{+}\right], p ; A_{+}\right)=\left\{\left(u, o_{+}\right) \mid \mathcal{B}_{+}\left(\left[z_{+}, w_{+}\right] ; A_{+}\right), u\left(o_{+}\right)=p\right\} .
$$

We now study the linearization of $\bar{\partial}_{\left(J^{+}, K^{+}\right)}$. First we describe the tangent space $T_{(u, p)} \mathcal{B}_{+}\left(\left[z_{+}, w_{+}\right] ; A_{+}\right)$. It decomposes

$$
T_{(u, p)} \mathcal{B}_{+}\left(\left[z_{+}, w_{+}\right] ; A_{+}\right)=T_{(u, p)}^{v} \mathcal{B}_{+}\left(\left[z_{+}, w_{+}\right] ; A_{+}\right) \oplus T_{(u, p)}^{h} \mathcal{B}_{+}\left(\left[z_{+}, w_{+}\right] ; A_{+}\right)
$$

into the vertical and horizontal components for the fibration

$$
\mathcal{B}_{+}\left(\left[z_{+}, w_{+}\right] ; A_{+}\right) \rightarrow M ;(u, p) \mapsto u .
$$

Then we have the canonical identification

$$
T_{(u, p)}^{v} \mathcal{B}_{+}\left(\left[z_{+}, w_{+}\right] ; A_{+}\right)=T_{(u)}^{v} \mathcal{B}_{+}\left(\left[z_{+}, w_{+}\right], p ; A_{+}\right) \cong W_{\alpha}^{1, p}\left(u^{*} T M ; p, z_{+}\right)
$$

where $W_{\alpha}^{1, p}\left(u^{*} T M ; p, z_{+}\right)$is the set of $\left(U, V_{\mathcal{R}_{1}(\lambda)}^{+}, V_{\mathbb{R}}^{+}\right)$satisfying the conditions given right above Remark 8.4.

On the other hand, the horizontal space is not canonically given and so we will choose them by prescribing their fiber components in the given trivialization of $\left(\left.E(M)\right|_{U} \cong U \times E(M, p)\right.$. Take a small convex neighborhood $U$ of $p$, and consider the parameterized line $\gamma:[0,1] \rightarrow U$ with $\gamma(0)=$ $p, \gamma(1)$ with constant speed. By the discussion before Proposition 8.1 we have diffeomorphisms $\varphi_{p \gamma(s)}:(M,\{p\}) \rightarrow(M, \gamma(s))$. We abbreviate $\varphi_{p \gamma(s)}$ 
by $\varphi_{s}$. Then the fiber component of the horizontal lifting of $v \in T_{p} M$ at $u$ in this trivialization is given by

$$
\left.\frac{d}{d s}\right|_{s=0} \varphi_{s} \circ u=: X_{0} \circ u,
$$

where $X_{s}$ is the vector field generating the isotopy $\varphi_{s}$ to the direction of $v=p \vec{p}^{\prime}$. Therefore using the local trivialization of $\mathcal{B}_{+}\left(\left[z_{+}, w_{+}\right] ; A_{+}\right) \rightarrow M$ induced by the family of diffeomorphisms $\varphi_{p p^{\prime}}$, the horizontal lifting of $v \in$ $T_{p} M$ is precisely $\left(X_{0} \circ u, v\right)$.

Note that the set of these variations $\left\{\left(X_{0} \circ u, v\right\}\right.$ defines an a $2 n$ dimensional subspace of $T_{u} \mathcal{B}_{+}\left(\left[z_{+}, w_{+}\right] ; A_{+}\right)$isomorphic to $T_{p} M$. We denote this subspace by $\widetilde{T}_{p} M \subset \mathcal{B}_{+}\left(\left[z_{+}, w_{+}\right] ; A_{+}\right)$.

Now we are ready to derive the formula for the linearization. When the variational vector field $U$ is tangent to a fixed target $M \backslash\{p\}$, the linearization $D_{u} \bar{\partial}_{\left(J^{+}, K^{+}\right)}(U)$ at $u$ is computed in a standard way.

Lemma 8.1. We have

$$
D_{u} \bar{\partial}_{\left(J^{+}, K^{+}\right)} U \in L_{\alpha}^{p}\left(\dot{\Sigma}, \Lambda_{J^{+}}^{0,1}\left(u^{*}(T M)\right)\right) .
$$

Proof. In the cylindrical end $(-\infty, 0] \times S^{2 n-1}$ in $M \backslash\{p\}$, the vector field

$$
U-\widetilde{U}=\chi_{+}(\tau) \operatorname{Pal}_{u(\tau, t)} U(-\infty, t)
$$

is asymptotically $J_{p}$-holomorphic: one way to see this is the following: identify $(-\infty, 0] \times S^{2 n-1}$ to $\mathbb{C}^{n} \backslash\{0\}$ and regard $u(\tau, t)$ in $\mathbb{C}^{n} \backslash\{0\}$. then the push forward of $\mathrm{Pal}_{u(\tau, t)} U(-\infty, t)$ is very close to the vector field

$$
V_{\mathbb{R}}^{+} u(z)+2 \pi e^{2 \pi\left(\tau-\tau_{+}+\sqrt{-1} t\right)} V_{\mathcal{R}_{1}(\lambda)}(0)
$$

in $\mathbb{C}^{n}$ when $\tau$ is negative enough. Furthermore, we have $\lim _{\tau \rightarrow-\infty} u(\tau, t)=p$, and $J(u(\tau, t)) \rightarrow J_{p}$. Therefore, we have

$$
\left|D_{u} \bar{\partial}_{\left(J^{+}, K^{+}\right)}(U-\widetilde{U})\right| \leq C e^{-c|\tau|}
$$

and so

$$
U=(U-\widetilde{U})+\widetilde{U} \in W_{\alpha}^{1, p}\left((-\infty, 0] \times S^{1}, \mathbb{R}\right) .
$$

This finishes the proof.

When the variational vector field is induced by a change of base point $p$ in $(M, p)$ in the direction of $v \in T_{p} M$, it is given by the one whose fiber component of the induced variational vector field at $u$ is given by $X_{0} \circ u$ where $X_{s}$ is the vector field generating the isotopy $\varphi_{s}$. We denote by $X_{v}$ the $X_{0}$ associated to $v \in T_{p} M$. Of course the component in $T_{p} M$ is just $v$. Note that the set of these variations $\left\{\left(X_{v} \circ u, v\right)\right\}$ defines an a $2 n$-dimensional subspace of $T_{(u, p)} \mathcal{B}_{+}\left(\left[z_{+}, w_{+}\right] ; A_{+}\right)$isomorphic to $T_{p} M$. We denote this subspace by $\widetilde{T_{p} M} \subset T_{(u, p)} \mathcal{B}_{+}\left(\left[z_{+}, w_{+}\right] ; A_{+}\right)$and $\left(X_{v} \circ u, v\right):=\widetilde{v}$. With this 
choice, obviously we have the decomposition

$$
\begin{aligned}
T_{(u, p)} \mathcal{B}_{+}\left(\left[z_{+}, w_{+}\right] ; A_{+}\right) & =T_{u} \mathcal{B}_{+}\left(\left[z_{+}, w_{+}\right], p ; A_{+}\right) \oplus \widetilde{T_{p} M} \\
& =W_{\alpha}^{1, p}\left(u^{*} T M ; p, z_{+}\right) \oplus \widetilde{T_{p} M} .
\end{aligned}
$$

Now the linearization of the section

$$
\bar{\partial}_{\left(J^{+}, K^{+}\right)}: \mathcal{B}_{+}\left(\left[z_{+}, w_{+}\right] ; A_{+}\right) \rightarrow \mathcal{L}_{+}\left(\left[z_{+}, w_{+}\right] ; A_{+}\right)
$$

at $(u, p)$ along

$$
\widetilde{v} \in \widetilde{T_{p} M} \subset T_{(u, p)} \mathcal{B}_{+}\left(\left[z_{+}, w_{+}\right] ; A_{+}\right)
$$

is given by $\left(D_{u} \bar{\partial}_{\left(J^{+}, K^{+}\right)}\left(X_{v_{+}} \circ u\right), v_{+}\right)$in the above-mentioned trivialization of $T_{(u, p)} \mathcal{B}_{+}\left(\left[z_{+}, w_{+}\right] ; A_{+}\right)$.

A straightforward calculation gives rise to the following:

Lemma 8.2. We have the formula

$$
\begin{aligned}
D_{u} \bar{\partial}_{\left(J^{+}, K^{+}\right)}\left(X_{v^{+}} \circ u\right)= & \left(u^{*} \nabla\right)^{(0,1)}\left(X_{v^{+}} \circ u\right)+T^{(0,1)}\left(d u, X_{v^{+}} \circ u\right) \\
& +D P_{K^{+}}(u)^{(0,1)}\left(X_{v^{+}} \circ u\right)
\end{aligned}
$$

where $T$ is the torsion tensor of the almost complex connection $\nabla$ and the $(0,1)$-parts are taken with respect to $J^{+}$.

Combining all these, we have obtained

Proposition 8.2. For $(u, p) \in \mathcal{M}_{1}\left(K_{-}, J_{-} ;\left[z_{+}, w_{+}\right] ; A_{+}\right)$,

$$
D_{(u, p)} \bar{\partial}_{\left(J^{+}, K^{+}\right)}: T_{(u, p)} W_{\alpha}^{1, p}\left(\dot{\Sigma}, M ; z_{+}\right) \rightarrow L_{\alpha}^{p}\left(\dot{\Sigma}, \Lambda_{J^{+}}^{0,1}\left(u^{*} T M\right)\right) \oplus T_{p} M
$$

is a Fredholm operator with

$$
\text { index } D_{u} \bar{\partial}_{\left(J^{+}, K^{+}\right)}=n-\mu\left(\left[z_{+}, w_{+}\right]\right)+2 c_{1}\left(A_{+}\right) .
$$

Next we prove the following transversality result of the section $\bar{\partial}_{\left(J^{+}, K^{+}\right)}$.

Proposition 8.3. For generic $J^{+} \in \mathcal{J}_{\omega} M$ and any $\left(J^{+}, K^{+}\right)$-holomorphic curve $u$,

$$
D_{(u, p)} \bar{\partial}_{\left(J^{+}, K^{+}\right)}: T_{(u, p)} W_{\alpha}^{1, p}\left(\dot{\Sigma}, M ; z_{+}\right) \rightarrow L_{\alpha}^{p}\left(\dot{\Sigma}, \Lambda_{J^{+}}^{0,1}\left(u^{*} T M\right)\right) \oplus T M
$$

is surjective.

Proof. We first consider $M \backslash\{p\}$ for a fixed $p$, and a fixed asymptote $\left(\gamma_{+}, \tau_{+}\right)$ in the cylindrical metric on $M \backslash\{p\}$ near the point $p$. For the linearization of

$$
\begin{aligned}
& \bar{\partial}_{\left(\cdot, K^{+}\right)}: W_{\alpha}^{1, p}\left(\dot{\Sigma}, M ; p, z_{+}, \gamma_{+}, \tau_{+}\right) \times \mathcal{J}_{\omega} \\
& \quad \bigcup_{u \in W_{\alpha}^{1, p}\left(\dot{\Sigma}, M ; p, z_{+}, \gamma_{+}, \tau_{+}\right)} \bigcup_{J^{+} \in \mathcal{J}_{\omega}} L_{\alpha}^{p}\left(\dot{\Sigma}, \Lambda_{J^{+}}^{0,1}\left(u^{*} T M\right)\right),
\end{aligned}
$$


standard argument shows that the map

$$
D_{u} \bar{\partial}_{\left(\cdot, K^{+}\right)}: T_{\left(u, J^{+}\right)}\left(W_{\alpha}^{1, p}\left(\dot{\Sigma}, M ; p, z_{+}, \gamma_{+}, \tau_{+}\right) \times \mathcal{J}_{\omega}\right) \rightarrow L_{\alpha}^{p}\left(\dot{\Sigma}, \Lambda_{J^{+}}^{0,1}\left(u^{*} T M\right)\right)
$$

is surjective for any given $\left(J^{+}, K^{+}\right)$-holomorphic curve $u$.

Now we enlarge the domain of $\bar{\partial}_{\left(\cdot, K^{+}\right)}$from $W_{\alpha}^{1, p}\left(\dot{\Sigma}, M ; p, z_{+}, \gamma_{+}, \tau_{+}\right) \times \mathcal{J}_{\omega}$ to $W_{\alpha}^{1, p}\left(\dot{\Sigma}, M ; z_{+}\right) \times \mathcal{J}_{\omega}$, i.e.,

$$
\begin{aligned}
\bar{\partial}_{\left(\cdot, K^{+}\right)}: W_{\alpha}^{1, p}\left(\dot{\Sigma}, M ; z_{+}\right) \times \mathcal{J}_{\omega} \\
\quad \rightarrow\left(\bigcup_{u \in W_{\alpha}^{1, p}\left(\dot{\Sigma}, M ; z_{+}\right)} \bigcup_{J^{+} \in \mathcal{J}_{\omega}} L_{\alpha}^{p}\left(\dot{\Sigma}, \Lambda_{J^{+}}^{0,1}\left(u^{*} T M\right)\right)\right) \oplus T M .
\end{aligned}
$$

Then for any given $\left(J^{+}, K^{+}\right)$-holomorphic curve $u \in W_{\alpha}^{1, p}\left(\dot{\Sigma}, M ; z_{+}\right)$,

$$
D_{u} \bar{\partial}_{\left(\cdot, K^{+}\right)}: T_{\left(u, J^{+}\right)}\left(W_{\alpha}^{1, p}\left(\dot{\Sigma}, M ; z_{+}\right) \times \mathcal{J}_{\omega}\right) \rightarrow L_{\alpha}^{p}\left(\dot{\Sigma}, \Lambda_{J^{+}}^{0,1}\left(u^{*} T M\right)\right) \oplus T M
$$

is surjective because (8.9) is surjective.

Now we consider the projection p: $W_{\alpha}^{1, p}\left(\dot{\Sigma}, M ; z_{+}\right) \times \mathcal{J}_{\omega} \rightarrow \mathcal{J}_{\omega}$. Then by Sard-Smale theorem, for any generic $J^{+} \in \mathcal{J}_{\omega}$, specifying to $J_{+}$for the above parameterized family of maps $D_{u} \bar{\partial}_{\left(\cdot, K^{+}\right)}$,

$$
D_{u} \bar{\partial}_{\left(J^{+}, K^{+}\right)}: T_{u} W_{\alpha}^{1, p}\left(\dot{\Sigma}, M ; z_{+}\right) \rightarrow L_{\alpha}^{p}\left(\dot{\Sigma}, \Lambda_{J^{+}}^{0,1}\left(u^{*} T M\right)\right) \oplus T M
$$

is surjective.

To prepare for the next subsection, we define the one-jet evaluation map for $\left(u, o_{+}\right) \in \mathcal{M}_{1}\left(\left[z_{+}, w_{+}\right] ; A_{+}\right)$. Recall that in the cylindrical coordinate chart near $p=u_{+}\left(o_{+}\right)$, we use the embedding

$$
\frac{1}{\delta}\left(\exp _{x}^{I}\right)^{-1}: B_{\varepsilon_{0}}(p) \backslash\{p\} \rightarrow\left(T_{p} M, J_{p}\right) \backslash\{p\} \cong\left(\mathbb{C}^{n}, J_{0}\right) \backslash\{0\} \cong \mathbb{R} \times S^{2 n-1}
$$

to express $u=(s, \Theta) \subset \mathbb{R} \times S^{2 n-1}$ with the asymptotes $\Theta(\tau, t) \rightarrow \gamma_{+}(t)$ and $s(\tau, t) \rightarrow 2 \pi\left(\tau-\tau_{+}\right)$. We define the tangential evaluation map

$$
\mathrm{ev}_{+}^{\#}: W_{\alpha}^{1, p}\left(\dot{\Sigma}, M ; z_{+}\right) \rightarrow \mathbb{R} \times \mathcal{R}_{1}(\lambda), \quad u \rightarrow\left(\tau_{+}, \gamma_{+}\right) .
$$

All the discussion above in this section hold for $\mathcal{M}_{1}\left(K^{-}, J^{-} ;\left[z_{-}, w_{-}\right] ; A_{-}\right)$ and $W_{\alpha}^{1, p}\left(\dot{\Sigma}, M ; z_{-}\right)$without change of proofs.

Here the $\alpha=\alpha(\tau)$ is a similar (but different) weighting function as before:

$$
\alpha(\tau)= \begin{cases}1, & \tau \leq 0, \\ \mathrm{e}^{2 \pi|\tau|}, & \tau \geq 0 .\end{cases}
$$

We abuse the notation $\alpha$ and the norm $\|\cdot\|_{1, p, \alpha}$ for $u_{-}$and $u_{+}$. 


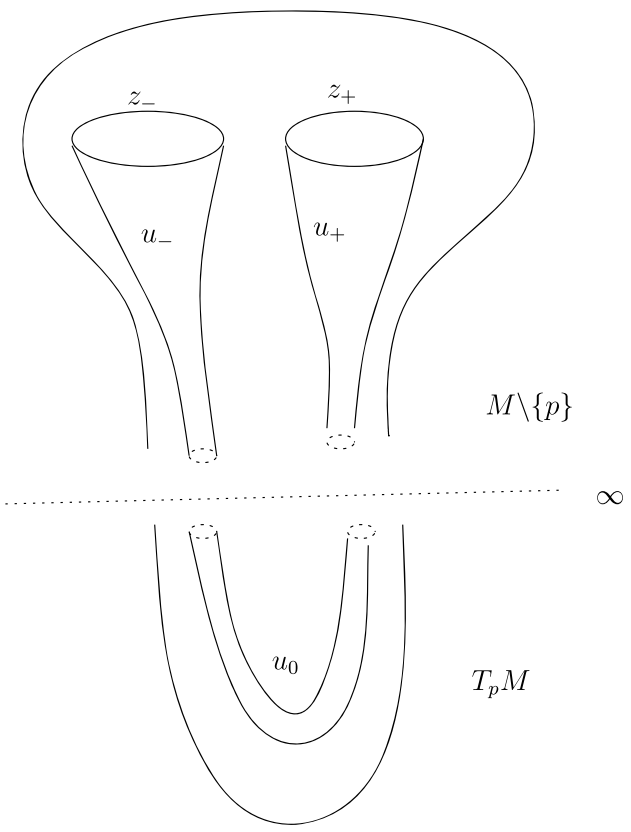

Figure 5. The enhanced nodal Floer trajectory.

The tangential evaluation map of $u \in W_{\delta}^{1, p}\left(\dot{\Sigma}, \widetilde{M} ; z_{-}\right)$is defined similarly up to the sign of $\tau_{-}$:

$$
e v_{-}^{\#}: W_{\delta}^{1, p}\left(\dot{\Sigma}, M ; z_{-}\right) \rightarrow \mathbb{R} \times \mathcal{R}_{1}(\lambda), \quad u \rightarrow\left(\tau_{-}, \gamma_{-}\right) .
$$

8.3. Off-shell formulation of enhanced nodal Floer trajectories. Now we are ready to define the Banach manifold hosting the enhanced nodal Floer trajectories (Figure 5). For notation brevity, we have set $T_{x} M \backslash\{0\}=$ $T_{x}^{+} M$ and $T M \backslash o_{M}=T^{+} M$, where $o_{M}$ is the zero section of $T M$. For all $x$ in $M$, identifying each $\left(T_{x} M, J_{x}\right)$ with $\left(\mathbb{C}^{n}, J_{0}\right)$, we get a family of inhomogeneous local models

$$
\mathcal{M}_{(0 ; 2,0)}^{+}\left(\dot{\Sigma}, T_{x} M\right) \cong \mathcal{M}_{(0 ; 2,0)}\left(\dot{\Sigma}, T_{x} M\right) \oplus T_{x}^{+} M,
$$

and the corresponding Banach manifolds hosting them

$$
\mathcal{B}_{x}:=W_{\delta,(0 ; 2,0)}^{1, p}\left(\dot{\Sigma}, T_{x} M\right) \oplus T_{x}^{+} M,
$$

and the Banach bundles

$$
\mathcal{L}_{x}:=\bigcup_{u \in \mathcal{B}_{x}} L_{\delta}^{p}\left(\dot{\Sigma}, \Lambda^{0,1}\left(u^{*} T\left(T_{x} M\right)\right) .\right.
$$

We emphasize that in defining $W_{\delta,(0 ; 2,0)}^{1, p}\left(\dot{\Sigma}, T_{x} M\right)$, the metric $h$ in the linear space $T_{x} M$ is cylindrical, like the one we defined in $\mathbb{C}^{n}$ in Section 6 : we let 
$h(z)=\lambda(z) g(x)$, where $g(x)$ is the original Riemannian metric on $T_{x} M$, $\lambda: T_{p} M \rightarrow \mathbb{R}_{+}$is the same radial function as in 6 , such that $\lambda(z)=\frac{1}{|z|_{g}^{2}}$ when $|z|_{g}$ is sufficiently large.

The union of these Banach manifolds, which hosts all local models in different $T_{x} M$, is

$$
\mathcal{B}_{\operatorname{lmd}}=\bigcup_{x \in M} \mathcal{B}_{x}:=\bigcup_{x \in M} W_{\delta,(1 ; 2,0)}^{1, p}\left(\dot{\Sigma}, T_{x} M\right) \oplus T_{x}^{+} M
$$

and the corresponding Banach bundle is

$$
\mathcal{L}_{\text {lmd }}=\bigcup_{x \in M} \mathcal{L}_{x}
$$

Here $T_{x}^{+} M$ is the summand encoding the vector $\vec{a}=\nabla f(x)$ which is not a zero vector since we assume that the node of the nodal Floer trajectories occur outside the set of critical points of the back-ground Morse function $f$. We also note that the group

$$
\operatorname{Aut}_{l m d}(x):=\left(T_{x} M \times(\mathbb{R} \backslash\{0\})\right) \times \mathbb{R}
$$

acts on $W_{\delta,(1 ; 2,0)}^{1, p}\left(\dot{\Sigma}, T_{x} M\right)$ where the factor $T_{x} M$ corresponds to the translations on $T_{x} M, \mathbb{R} \backslash\{0\}$ corresponds to multiplication by nonzero real constant on $T_{x} M$ and the last $\mathbb{R}$-factor corresponds to the domain $\tau$-translations. This action also induces an action on $\mathcal{L}_{\text {lmd }}$.

We let

$$
\pi: \bigcup_{x \in M} \mathcal{M}_{(0 ; 2,0)}^{+}\left(\dot{\Sigma}, T_{x} M\right) \rightarrow M
$$

be the projection to the base $M$, and the symplectic field theory evaluation map

$$
\begin{aligned}
e v^{\mathrm{SFT}} & =\left(e v_{-}^{\mathrm{SFT}}, e v_{+}^{\mathrm{SFT}}\right): \mathcal{M}_{(0 ; 2,0)}^{+}\left(\dot{\Sigma}, T_{x} M\right) \rightarrow \mathcal{R}_{1}(\lambda) \times_{\Delta_{M}} \mathcal{R}_{1}(\lambda), \\
u_{0} & \rightarrow\left(\gamma_{0-}, \gamma_{0+}\right),
\end{aligned}
$$

where $\gamma_{0 \pm}$ are the asymptotic data of the local model $u_{0}$. Here we use the fiber product $\mathcal{R}_{1}(\lambda) \times_{\Delta_{M}} \mathcal{R}_{1}(\lambda)$ to emphasize that $\gamma_{-}$and $\gamma_{+}$lie in the same $T_{p} M$, where $p=\pi\left(u_{0}\right)$. (We recall from Subsection 8.1 and the paragraph right therebefore that we have made the identification of $\left(T_{p} M, J_{p}, \omega_{p}\right)$ and $\mathbb{C}^{n}$ using the Darboux family $I$.)

We form the Banach manifold hosting enhanced nodal Floer trajectories via the fiber product of

$$
\begin{gathered}
\pi_{\Theta} \circ e v_{-}^{\#} \times \pi_{\Theta} \circ e v_{\mathrm{SFT}} \times \pi_{\Theta} e v_{+}^{\#}: \mathcal{R}_{1}(\lambda) \times\left(\mathcal{R}_{1}(\lambda) \times_{\Delta_{M}} \mathcal{R}_{1}(\lambda)\right) \times \mathcal{R}_{1}(\lambda) \\
\left(u_{-}, u_{0}, u_{+}\right) \rightarrow\left(\pi_{\Theta} \circ e v_{-}^{\#}\left(u_{-}\right), e v_{\mathrm{SFT}}\left(u_{0}\right), \pi_{\Theta} \circ e v_{+}^{\#}\left(u_{+}\right)\right):
\end{gathered}
$$


Let $\Delta_{\mathcal{R}_{1}(\lambda)} \subset \mathcal{R}_{1}(\lambda) \times \mathcal{R}_{1}(\lambda)$ be the diagonal. Then we define

$$
\begin{aligned}
\mathcal{B}_{\text {nodal }}:= & \left(\pi_{\Theta} \circ e v_{-}^{\#} \times e v_{\mathrm{SFT}} \times \pi_{\Theta} \circ e v_{+}^{\#}\right)^{-1}\left(\Delta_{\mathcal{R}_{1}(\lambda)} \times_{\Delta_{M}} \Delta_{\mathcal{R}_{1}(\lambda)}\right) \\
= & \left\{\left(u_{-}, u_{0}, u_{+}\right) \in \mathcal{B}_{-} \times \mathcal{B}_{\text {lmd }} \times \mathcal{B}_{+} \mid u_{-}\left(o_{-}\right)=u_{+}\left(o_{+}\right)\right. \\
& \left.\pi_{\Theta} \circ e v_{-}^{\#}\left(u_{-}\right)=e v_{\mathrm{SFT}}^{-}\left(u_{0}\right), \pi_{\Theta} \circ e v_{+}^{\#}\left(u_{+}\right)=e v_{\mathrm{SFT}}^{+}\left(u_{0}\right)\right\}
\end{aligned}
$$

to be the Banach manifold. Due to the action of $\left(T_{x} M \times(\mathbb{R} \backslash\{0\})\right) \times \mathbb{R}$ on $\mathcal{B}_{\text {lmd }}$, the same group acts on $\mathcal{B}_{\text {nodal }}$.

From the matching condition it is clear that for any $u=\left(u_{-}, u_{0}, u_{+}\right) \in$ $\mathcal{B}_{\text {nodal }}$, its tangent space is

$$
\begin{aligned}
T_{u} \mathcal{B}_{\text {nodal }}= & \left\{\left(\xi_{-}, \xi_{0}, \xi_{+}\right) \in T_{u_{+}} \mathcal{B}_{+} \times T_{u_{0}} \mathcal{B}_{\text {lmd }} \times T_{u_{-}} \mathcal{B}_{-} \mid\right. \\
& \left.v_{-}=v_{+}=v_{0}=v, V_{\mathcal{R}_{1}(\lambda)}^{ \pm}=V_{\mathcal{R}_{1}(\lambda)}^{0 \pm}\right\}
\end{aligned}
$$

where we have the expressions

$$
\begin{aligned}
& \xi_{ \pm}=\left(U_{ \pm}, V_{\mathcal{R}_{1}(\lambda)}^{ \pm}, V_{\mathbb{R}}^{ \pm}, v_{ \pm}\right) \in T_{u_{ \pm}} \mathcal{B}_{ \pm} \\
& \xi_{0}=\left(U_{0}, V_{\mathcal{R}_{1}(\lambda)}^{0-}, V_{\mathbb{R}}^{0-}, V_{\mathcal{R}_{1}(\lambda)}^{0+}, V_{\mathbb{R}}^{0+}, v_{0}\right) \in T_{u_{0}} \mathcal{B}_{\text {lmd }},
\end{aligned}
$$

and the $v_{0} \in T_{p} M$ correspond to the variation of the base point $p$.

We have a natural $\operatorname{Aut}_{\text {lmd }}(x)$-equivariant section

$$
\begin{aligned}
\bar{\partial}_{(J, K, f)}: \mathcal{B}_{\text {nodal }} \rightarrow \mathcal{L}_{-} \times \mathcal{L}_{\text {lmd }} \times \mathcal{L}_{+}, \\
\quad\left(u_{-}, u_{0}, u_{+}, p\right) \rightarrow\left(\bar{\partial}_{\left(J^{-}, K^{-}\right)} u_{-}, \bar{\partial}_{\left(J_{p}, f\right)} u_{0}, \bar{\partial}_{\left(J^{+}, K^{+}\right)} u_{+}\right),
\end{aligned}
$$

where $p=u_{ \pm}\left(o_{ \pm}\right)$is the nodal point, and $\bar{\partial}_{\left(J_{p}, f\right)} u_{0}=\bar{\partial}_{J_{p}} u_{0}-\nabla f(p) d \tau+$ $J_{p} \nabla f(p) d t$.

If we put more topological restrictions on $u_{-}$and $u_{+}$, namely if we let $\mathcal{B}_{\text {nodal }}\left(\left[z_{-}, w_{-}\right],\left[z_{+}, w_{+}\right] ; A_{-}, A_{+}\right)=\left\{\left(u_{-}, u_{0}, u_{+}\right) \in \mathcal{B}_{\text {nodal }} \mid\left[u_{ \pm} \# w_{ \pm}\right]=A_{ \pm}\right\}$, then similarly we have the section

$$
\begin{aligned}
\bar{\partial}_{(J, K, f)} & : \mathcal{B}_{\text {nodal }}\left(\left[z_{-}, w_{-}\right],\left[z_{+}, w_{+}\right] ; A_{-}, A_{+}\right) \\
\longrightarrow & \mathcal{L}\left(\left[z_{-}, w_{-}\right] ; A_{-}\right) \times \mathcal{L}_{\text {lmd }} \times \mathcal{L}\left(\left[z_{+}, w_{+}\right] ; A_{+}\right),
\end{aligned}
$$

and the moduli space of enhanced nodal Floer trajectories with the background Morse function $f$ defined in Subsection 5.1 can be interpreted as

$$
\mathcal{M}^{\text {nodal }}\left(\left[z_{-}, w_{-}\right],\left[z_{+}, w_{+}\right] ; A_{-}, A_{+} ;(K, J),\left(f, J_{0}\right)\right)=\left(\bar{\partial}_{(J, K, f)}\right)^{-1}(0)
$$

from (8.18).

Proposition 8.4. For generic $J^{-}$and $J^{+}$in $\mathcal{J}_{\omega}$, any enhanced nodal Floer trajectory $u=\left(u_{-}, u_{0}, u_{+}\right) \in \mathcal{M}^{\text {nodal }}\left(\left[z_{-}, w_{-}\right],\left[z_{+}, w_{+}\right] ; A_{-}, A_{+} ;(K, J),(f\right.$, $\left.J_{0}\right)$ ) is regular, in the sense that

$$
\begin{aligned}
& D_{(u, p)} \bar{\partial}_{(J, K, f)}: T_{(u, p)} \mathcal{B}_{\text {nodal }}\left(\left[z_{-}, w_{-}\right],\left[z_{+}, w_{+}\right] ; A_{-}, A_{+}\right) \\
& \quad \longrightarrow \mathcal{L}\left(\left[z_{-}, w_{-}\right] ; A_{-}\right) \times \mathcal{L}_{\text {lmd }} \times \mathcal{L}\left(\left[z_{+}, w_{+}\right] ; A_{+}\right) \oplus T_{p} M
\end{aligned}
$$


is surjective. Consequently, there exists a right inverse of $D_{(u, p)} \bar{\partial}_{(J, K, f)}$,

$$
\begin{aligned}
& \left.Q^{\varepsilon}\right|_{\varepsilon=0}: \quad \mathcal{L}\left(\left[z_{-}, w_{-}\right] ; A_{-}\right) \times \mathcal{L}_{\text {lmd }} \times \mathcal{L}\left(\left[z_{+}, w_{+}\right] ; A_{+}\right) \oplus T_{p} M \\
& \quad \longrightarrow T_{(u, p)} \mathcal{B}_{\text {nodal }}\left(\left[z_{-}, w_{-}\right],\left[z_{+}, w_{+}\right] ; A_{-}, A_{+}\right) .
\end{aligned}
$$

Before the proof of the proposition, we state a lemma concerning the linearization of the inhomogeneous local model equation with respect to the variation $\widetilde{v}=(0, v) \in T_{\left(u_{0}, p\right)} \mathcal{B}_{\text {lmd }}$ which is the horizontal lifting of $v \in T_{p} M$ as constructed before for the curves $u_{ \pm}$.

Lemma 8.3. For any $\left(u_{0}, v\right) \in \mathcal{M}_{(0 ; 2,0)}^{+}\left(\dot{\Sigma}, T_{x} M\right)$ satisfying

$$
\bar{\partial}_{\left(J_{p}, f\right)} u_{0}=\bar{\partial}_{J} u_{0}-\nabla f(p) d \tau+J \nabla f(p) d t=0
$$

and corresponding to the variation $\widetilde{v}=\left(X_{v} \circ u_{0}, v\right)$ in the trivialization constructed by the family $\varphi_{p p^{\prime}}$ with $v \in T_{p} M$, we have

$$
\left(D_{\left(u_{0}, p\right)} \bar{\partial}_{\left(J_{p}, f\right)}(\widetilde{v})\right)^{v} \in \mathcal{L}_{\text {lmd }}
$$

Consequently, the map

$$
D_{\left(u_{0}, p\right)} \bar{\partial}_{\left(J_{p}, f\right)}: T_{u_{0}} \mathcal{B}_{\text {lmd }} \rightarrow \mathcal{L}_{\operatorname{lmd}} \oplus T_{p} M
$$

is surjective.

Now we go back to the proof of the Proposition 8.4:

Proof. Let $u=\left(u_{+}, u_{0}, u_{-}\right)$be an enhanced nodal Floer trajectory with the nodal point $p$. In Subsection 8.2, we have proved that for generic $J^{ \pm} \in \mathcal{J}_{\omega}$,

$$
D_{\left(u_{ \pm}, p\right)} \bar{\partial}_{\left(J^{ \pm}, K^{ \pm}\right)}: T_{\left(u_{ \pm}, p\right)} \mathcal{B}\left(\left[z_{ \pm}, w_{ \pm}\right] ; A_{ \pm}\right) \rightarrow L_{\delta}^{p}\left(\dot{\Sigma}, \Lambda_{J_{ \pm}}^{0,1}\left(u_{ \pm}^{*}(T M)\right)\right) \oplus T_{p} M
$$

is surjective. By Proposition 5.4, for generic $J^{ \pm} \in \mathcal{J}_{\omega}$ we have that

$$
\begin{aligned}
& e v_{-} \times e v_{+}: \mathcal{M}_{1}\left(J^{-}, K^{-} ;\left[z_{-}, w_{-}\right] ; A_{-}\right) \times \mathcal{M}_{1}\left(J^{+}, K^{+} ;\left[z_{+}, w_{+}\right] ; A_{+}\right), \\
& \left(u_{-}, u_{+}\right) \rightarrow\left(u_{-}\left(o_{-}\right), u_{+}\left(o_{+}\right)\right)
\end{aligned}
$$

is transversal to $\Delta_{M} \subset M \times M$. Therefore, for any $\eta_{ \pm} \in L_{\delta}^{p}\left(\dot{\Sigma}, \Lambda_{J_{ \pm}}^{0,1}\left(u_{ \pm}^{*}(T M)\right)\right)$, there exist $\xi_{ \pm}=\left(U_{ \pm}, V_{\mathcal{R}_{1}(\lambda)}^{ \pm}, V_{\mathbb{R}}^{ \pm}, v_{ \pm}\right) \in T_{u_{ \pm}} \mathcal{B}\left(\left[z_{ \pm}, w_{ \pm}\right] ; A_{ \pm}\right)$such that

$$
D_{u_{ \pm}} \bar{\partial}_{\left(J^{ \pm}, K^{ \pm}\right)} \xi_{ \pm}=\eta_{ \pm} \quad \text { and } \quad v_{-}=v_{+}:=v \text {. }
$$

Then for any $\eta_{0} \in \Lambda_{J_{p}}^{0,1}\left(u_{0}^{*}\left(T T_{p} M\right)\right)$, by the transversality of the local models in $\mathbb{C}^{n} \cong T_{p} M$, for $u_{0}$ in $T_{p} M$, there exists

$$
\xi_{0}=\left(U_{0}, V_{\mathcal{R}_{1}(\lambda)}^{0-}, V_{\mathcal{R}_{1}(\lambda)}^{0+}\right) \in T_{u_{0}} \mathcal{B}_{p}
$$

such that $D_{u_{0}} \bar{\partial}_{\left(J_{p}, f\right)} \xi_{0}=\eta_{0}$. Hence for $\left(\xi_{0}, v_{0}\right) \in T_{\left(u_{0}, v_{0}\right)} \mathcal{B}_{\text {lmd }}$ with $v_{0}=v$,

$$
D_{u_{0}} \bar{\partial}_{\left(J_{p}, f\right)}\left(\xi_{0}, v_{0}\right)=D_{u_{0}} \bar{\partial}_{J_{p}, f} \xi_{0}=\eta_{0} .
$$


The proposition immediately follows from the surjectivity of $D_{u_{ \pm}} \bar{\partial}_{\left(J_{ \pm}, K_{ \pm}\right)}$, $D_{u_{0}} \bar{\partial}_{\left(J_{p}, f\right)}$ and the transversality of the evaluation map

$$
\pi_{\Theta} \circ e v_{-}^{\#} \times \pi_{\Theta} \circ e v_{+}^{\#} \rightarrow \mathcal{R}_{1}(\lambda) \times \mathcal{R}_{1}(\lambda)
$$

to the diagonal $\Delta_{\mathcal{R}_{1}(\lambda)}$. For any $\eta=\left(\eta_{-}, \eta_{0}, \eta_{+}\right)$, using the above construction we define $\left.Q^{\varepsilon}\right|_{\varepsilon=0}$ is defined to be $\left.Q^{\varepsilon}\right|_{\varepsilon=0}(\eta)=\left(\xi_{-}, \xi_{0}, \xi_{+}, v\right)$. Then $\left.Q^{\varepsilon}\right|_{\varepsilon=0}$ is a right inverse of $D_{(u, p)} \bar{\partial}_{(J, K, f)}$ and the proposition follows.

Remark 8.5. For the purpose of constructing approximate right inverse later, we define the operators $Q_{-}, Q_{0}$ and $Q_{+}$by

$$
Q_{-} \eta_{-}:=\xi_{-}, \quad Q_{0} \eta_{0}:=\xi_{0}, \quad Q_{+} \eta_{+}:=\xi_{+}
$$

for the $\left(\eta_{-}, \eta_{0}, \eta_{+}\right)$and $\left(\xi_{-}, \xi_{0}, \xi_{+}\right)$above.

8.4. Appendix: Explosion. In this appendix, we collect various facts on the so called, explosion construction of manifolds. We verbatim follow the exposition in the smooth context given by Weinstein in Section 4 [We2].

Let $Y \subset X$ be a submanifold. The explosion of $X$ along $Y$, denoted by $E(X, Y)$, is defined by beginning with the product $X \times \mathbb{R}$, removing the "axis" $X \times\{0\}$, and replacing it with the normal bundle $N(X, Y)=$ $T_{Y} X / T Y$ to $Y$ in $X$. The differentiable structure on $E(X, Y)$ is taken to be the usual product structure on $X \times(\mathbb{R} \times\{0\})$. Charts near $X \times\{0\}$ are defined with the aid of local coordinates on $X$. A more precise description of local charts near $X \times\{0\}$ is in order.

Let $X$ have dimension $n$ and $Y$ have dimension $k$. We abbreviate the coordinates $\left(x_{1}, \ldots, x_{n}\right)$ on $\mathbb{R}^{n}$ by $(y, z)$, where $y=\left(x_{1}, \ldots, x_{k}\right)$ and $z=$ $\left(x_{k+1}, \ldots, x_{n}\right)$. Suppose that $\Phi$ is a submanifold fold chart defined on an open subset $U \subset X$ at $p \in Y \subset X$, i.e., a chart for the pair $(X, Y)$ defined on an open subset $\mathcal{U}$ of $\left(\mathbb{R}^{n}, \mathbb{R}^{k}\right)$ which is invariant under the retraction $(y, z) \mapsto(y, 0)$. The corresponding chart $E(\Phi)$ for $E(X, Y)$ is defined on the open subset $\left\{\left(y, z^{\prime}, \varepsilon\right) \mid\left(y, \varepsilon z^{\prime}\right) \in \mathcal{U}\right\}$ of $\mathbb{R}^{n+1}$ by

$$
E(\Phi)\left(y, z^{\prime}, \varepsilon\right)=\left(\Phi\left(y, \varepsilon z^{\prime}\right), \varepsilon\right)
$$

for $\varepsilon \neq 0$ with $E(\Phi)\left(y, z^{\prime}, 0\right)$ defined as the projection of the tangent vector $T_{(y, 0)} \Phi\left(0, z^{\prime}\right)$ into the normal bundle $N(X, Y)$. The following theorem is proved by Weinstein [We2].

Theorem 8.1 (Lemma 4.3, [We2]). Let $f:(X, Y) \rightarrow(Z, W)$ be a smooth mapping. Then it uniquely induces a smooth mapping $E(f): E(X, Y) \rightarrow$ $E(Z, W)$ such that

(1) $E(f)$ extends to the restriction $f: X \backslash Y \rightarrow Z \backslash W$, 
(2) When $\Phi$ and $\Psi$ are local charts of $(X, Y)$ and $(Z, W)$ respectively and the local representative $\Psi^{-1} f \Phi$ is written as $\Psi^{-1} f \Phi=(g, h)$ : $\left(\mathbb{R}^{n}, \mathbb{R}^{k}\right) \rightarrow\left(R^{m}, \mathbb{R}^{\ell}\right)$, then the local representation $E(g, h)$ of $E(f)$ with respect to the charts $E(\Phi)$ and $E(\Psi)$ is given for $\varepsilon \neq 0$ by the "partial difference quotient"

$$
E(g, h)\left(y, z^{\prime}, e\right)=\left(g\left(y, \varepsilon z^{\prime}\right),(1 / \varepsilon) h\left(y, \varepsilon z^{\prime}\right), \varepsilon\right)
$$

and for $\varepsilon=0$ by the normal derivative

$$
E(g, h)\left(y, z^{\prime}, 0\right)=\left(g(y, 0),\left(\partial h / \partial z^{\prime}\right)(y, 0), 0\right) .
$$

Two immediate consequences are also derived in [We2].

Corollary 8.1 (Theorem 4.4, [We2]). Assuming to each pair $(X, Y)$ the exploded manifold $E(X, Y)$ with the differentiable structure described above defines a covariant functor from the category of pairs of manifolds to the category of manifolds over $\mathbb{R}$.

Corollary 8.2. The identity map on $X \times(\mathbb{R} \backslash\{0\})$ extends to a unique smooth mapping from $E(X, Y)$ to $X \times \mathbb{R}$. The restriction of this mapping to $N(X, Y)$ is the bundle projection onto $Y \times\{0\}$.

This corollary defines a canonical smooth projection map $E(X, Y) \rightarrow$ $X \times \mathbb{R}$, which we denote by $\pi_{E(X, Y)}$.

\section{Part II. Analysis: scale-dependent gluing and compactification}

\section{Smoothing of nodal Floer trajectories I; to "disk-flow-disk"}

The disk-flow-disk moduli spaces

$$
\mathcal{M}^{\text {para }}\left(\left[z_{-}, w_{-}\right] ; f ;\left[z_{+}, w_{+}\right] ; A_{ \pm}\right), \quad \mathcal{M}^{\varepsilon}\left(\left[z_{-}, w_{-}\right] ; f ;\left[z_{+}, w_{+}\right] ; A_{ \pm}\right)
$$

have been defined in Section 4. Recall that the moduli space of "disk-flowdisk" elements of flow time $\varepsilon$ is

$$
\begin{aligned}
\mathcal{M}^{\varepsilon}\left(\left[z_{-}, w_{-}\right] ; f ;\left[z_{+}, w_{+}\right] ; A_{ \pm}\right):= & \left\{\left(u_{-}, \chi, u_{+}\right) \mid u_{ \pm} \in \mathcal{M}\left(K^{ \pm}, J^{ \pm} ; \vec{z}_{ \pm} ; A_{ \pm}\right)\right. \\
& \chi:[0, \varepsilon] \rightarrow M, \dot{\chi}-\nabla f(\chi)=0, u_{-}\left(o_{-}\right) \\
& \left.=\chi(0), u_{+}\left(o_{+}\right)=\chi(\varepsilon)\right\} .
\end{aligned}
$$

We give $\mathcal{M}^{\varepsilon}\left(\left[z_{-}, w_{-}\right] ; f ;\left[z_{+}, w_{+}\right] ; A_{ \pm}\right)$another interpretation through evaluation maps. This point of view is more suitable for analyzing the transition from nodal Floer trajectories to "disk-flow-disk" elements. Consider the deformed evaluation map

$$
\begin{aligned}
& \phi_{f}^{\varepsilon} e v_{-} \times e v_{+}: \mathcal{M}_{1}\left(\left[z_{-}, w_{-}\right] ; A_{-}\right) \times \mathcal{M}_{1}\left(\left[z_{+}, w_{+}\right] ; A_{+}\right) \rightarrow M \times M \\
& \quad\left(u_{-}, u_{+}\right) \rightarrow\left(\phi_{f}^{\varepsilon} u_{-}\left(o_{-}\right), u_{+}\left(o_{+}\right)\right),
\end{aligned}
$$


where $\phi_{f}^{\varepsilon}: M \rightarrow M$ is the time- $\varepsilon$ flow of the Morse function $f$. Then it is easy to see

$$
\begin{gathered}
\mathcal{M}^{\varepsilon}\left(\left[z_{-}, w_{-}\right] ; f ;\left[z_{+}, w_{+}\right] ; A_{ \pm}\right)=\left\{\left(u_{-}, \chi, u_{+}\right) \mid\right. \\
\left.\left(u_{-}, u_{+}\right) \in\left(\phi_{f}^{\varepsilon} e v_{-} \times e v_{+}\right)^{-1}(\Delta), \chi(\tau)=\phi_{f}^{\tau} u_{-}\left(o_{-}\right) \text {for } 0 \leq \tau \leq \varepsilon\right\} .
\end{gathered}
$$

Using the above interpretation, if

$$
\phi_{f}^{\varepsilon} e v_{-} \times e v_{+} \text {is transversal to } \Delta \subset M \times M
$$

and if $D_{u_{ \pm}} \bar{\partial}_{\left(K_{ \pm}, J_{ \pm}\right)}$are surjective, then by the inverse function theorem for (9.1), $\mathcal{M}^{\varepsilon}\left(\left[z_{-}, w_{-}\right] ; f ;\left[z_{+}, w_{+}\right] ; A_{ \pm}\right)$is a manifold of dimension

$$
\begin{aligned}
\operatorname{dim} & \mathcal{M}^{\varepsilon}\left(\left[z_{-}, w_{-}\right] ; f ;\left[z_{+}, w_{+}\right] ; A_{ \pm}\right) \\
\quad & =\operatorname{dim} \mathcal{M}\left(\left[z_{-}, w_{-}\right] ; A_{-}\right)+\operatorname{dim} \mathcal{M}\left(\left[z_{+}, w_{+}\right] ; A_{+}\right)-2 n \\
& =\left(n+\mu_{C Z}\left(\left[z_{-}, w_{-}\right]\right)+2 c_{1}\left(A_{-}\right)\right)+\left(n-\mu_{C Z}\left(\left[z_{+}, w_{+}\right]\right)+2 c_{1}\left(A_{+}\right)\right)-2 n \\
& =\mu_{C Z}\left(\left[z_{-}, w_{-}\right]\right)-\mu_{C Z}\left(\left[z_{+}, w_{+}\right]\right)+2 c_{1}\left(A_{-}\right)+2 c_{1}\left(A_{+}\right),
\end{aligned}
$$

if $\varepsilon>0$ is sufficiently small.

Assumption: For transversality argument, from now on we assume the critical points of $f$ do not coincide with the nodal points of nodal Floer trajectories, this can be achieved by a generic $f$.

Since we mainly care about the transition from disk-flow-disk elements to resolved nodal Floer trajectories during the PSS cobordism, only the disk-flow-disk elements with short-time flows will be considered. So we fix a sufficiently small $\varepsilon_{0}>0$ which is to be determined later, and consider the "disk-flow-disk" moduli spaces $\mathcal{M}^{\varepsilon}\left(\left[z_{-}, w_{-}\right] ; f ;\left[z_{+}, w_{+}\right] ; A_{ \pm}\right)$with $0 \leq$ $\varepsilon \leq \varepsilon_{0}$. The $\varepsilon=0$ case corresponds to the moduli space of nodal Floer trajectories.

Lemma 9.1. Suppose that the almost complex structures $J^{ \pm}$are generically chosen so for any nodal Floer trajectory $\left(u_{-}, u_{+}\right), D_{u_{ \pm}} \bar{\partial}_{\left(K_{ \pm}, J_{ \pm}\right)}$are surjective, and $u_{-}\left(o_{-}\right)$and $u_{+}\left(o_{+}\right)$are immersed points as in Theorem 1.2. Then there exists $\varepsilon_{0}>0$, such that for any $\left(u_{-}, \chi, u_{+}\right) \in \mathcal{M}^{\varepsilon}\left(\left[z_{-}, w_{-}\right] ; f ;\left[z_{+}, w_{+}\right] ; A_{ \pm}\right)$ where $\varepsilon \in\left[0, \varepsilon_{0}\right]$, the above property is preserved, i.e. $D_{u_{ \pm}} \bar{\partial}_{\left(K_{ \pm}, J_{ \pm}\right)}$are surjective, and $u_{-}\left(o_{-}\right)$and $u_{+}\left(o_{+}\right)$are immersed points.

Proof. We prove that $u_{-}\left(o_{-}\right)$and $u_{+}\left(o_{+}\right)$are immersed points. Otherwise, there exist $\varepsilon_{i} \rightarrow 0$, and $\left(u_{-}^{i}, \chi_{i}, u_{+}^{i}\right) \in \mathcal{M}^{\varepsilon_{i}}\left(K^{ \pm}, J^{ \pm} ;\left[z_{-}, w_{-}\right], f,\left[z_{+}, w_{+}\right] ; A_{ \pm}\right)$, such that at least one of $u_{-}^{i}\left(o_{-}\right)$and $u_{+}^{i}\left(o_{+}\right)$is not an immersed point. Passing to a subsequence we may assume, say $d u_{-}^{i}\left(o_{-}\right)$, is 0 for any $i$. Since the energy of any curves $u_{ \pm}^{i}$ is uniformly bounded due to the boundary condition, we can take a subsequence again and get a limiting nodal curve $\left(u_{-}^{\infty}, u_{+}^{\infty}\right)$ by Gromov-compactness. The images of $\chi_{i}$ converge to the nodal point. No bubbling can occur on $u_{-}^{\infty}$ or $u_{+}^{\infty}$, because if a bubble occurs on $u_{+}^{\infty}$ or $u_{+}^{\infty}$, 
then by the semi-positive condition and the genericity of $J^{ \pm}$, we can resolve the bubble to get a at least two-dimensional family of nodal Floer trajectories, contradicting with the rigidity assumption on nodal Floer trajectories. Therefore, the curves $\left(u_{-}^{i}, u_{+}^{i}\right)$ converge to $\left(u_{-}^{\infty}, u_{+}^{\infty}\right)$ in $C^{1}$ topology. This implies $d u_{-}^{\infty}\left(o_{-}\right)=\lim _{i \rightarrow \infty} d u_{-}^{i}\left(o_{-}\right)=0$, contradicting with the immersion condition at the nodal point.

Since $u_{ \pm}\left(o_{ \pm}\right)$are immersed points, $u_{ \pm}$are somewhere injective. Then the genericity of $J^{ \pm}$implies that $D_{u_{ \pm}} \bar{\partial}_{\left(K_{ \pm}, J_{ \pm}\right)}$are surjective.

To complete the PSS cobordism from "disk-flow-disk" configurations to nodal Floer trajectories, we will build a collar neighborhood of

$$
\mathcal{M}^{0}\left(\left[z_{-}, w_{-}\right], f,\left[z_{+}, w_{+}\right] ; A_{ \pm}\right)
$$

in $\mathcal{M}^{\text {para }}\left(\left[z_{-}, w_{-}\right] ; f ;\left[z_{+}, w_{+}\right] ; A_{ \pm}\right)$; More precisely, for some $\varepsilon_{0}>0$, we will construct a differentiable map

$$
\begin{aligned}
& G: \mathcal{M}^{0}\left(\left[z_{-}, w_{-}\right], f,\left[z_{+}, w_{+}\right] ; A_{ \pm}\right) \times\left[0, \varepsilon_{0}\right) \rightarrow \mathcal{M}^{\text {para }}\left(\left[z_{-}, w_{-}\right] ;\right. \\
& \left.\quad f ;\left[z_{+}, w_{+}\right] ; A_{ \pm}\right)
\end{aligned}
$$

such that for each $\varepsilon \in\left[0, \varepsilon_{0}\right)$,

$G_{\varepsilon}:=G(\cdot, \varepsilon): \mathcal{M}^{0}\left(\left[z_{-}, w_{-}\right], f,\left[z_{+}, w_{+}\right] ; A_{ \pm}\right) \rightarrow \mathcal{M}^{\varepsilon}\left(\left[z_{-}, w_{-}\right] ; f ;\left[z_{+}, w_{+}\right] ; A_{ \pm}\right)$ is a diffeomorphism. This problem is reduced to the following finitedimensional differential topology lemma:

Lemma 9.2. $X, Y, Z$ are differentiable manifolds, and only $X$ may have boundary. $X$ is compact, $Z$ is a differentiable submanifold in $Y$, and $I$ is an interval containing 0 . Let $\Phi: X \times I \rightarrow Y$ be a differentiable map. Denote $\Phi_{\varepsilon}:=\Phi(\cdot, \varepsilon)$ for $\varepsilon \in$ I. If $\Phi_{0}: X \rightarrow Y$ is transversal to $Z$ with nonempty intersection, and $\Phi_{0}(\partial X) \cap Z=\emptyset$, then

(1) There exists $\varepsilon_{0}>0$, such that for any $\varepsilon \in\left[0, \varepsilon_{0}\right], \Phi_{\varepsilon}$ is transversal to $Z$;

(2) Furthermore, there exits a differentiable map $G: \Phi_{0}^{-1}(Z) \times\left[0, \varepsilon_{0}\right] \rightarrow$ $\Phi^{-1}(Z)$, such that for any $\varepsilon \in\left[0, \varepsilon_{0}\right], G_{\varepsilon}:=G(\cdot, \varepsilon)$ gives a diffeomorphism from $\Phi_{0}^{-1}(Z)$ to $\Phi_{\varepsilon}^{-1}(Z)$.

Proof. Since $X$ is compact, the compact-open topology in the function space $C^{1}(X, Y)$ coincides with the strong topology $C_{S}^{1}(X, Y)$ as in [Hir]. Since the set of maps transversal to $Z$ in $C_{S}^{1}(X, Y)$ is open, by the condition of $\Phi$ and $\Phi_{0}$ we conclude that for $\varepsilon_{0}$ sufficiently small,

$$
\text { for all } \varepsilon \in\left[0, \varepsilon_{0}\right], \Phi_{\varepsilon} \text { is transversal to } Z \text {. }
$$

Since $\Phi_{\varepsilon}(X) \cap Y \neq \emptyset$, and $\Phi_{0}(\partial X) \cap Y=\emptyset$, the pre-image of all intersections lies in $\operatorname{int}(X)$, where $\operatorname{int}(X)$ is the interior of $X$. Therefore for $\varepsilon_{0}$ sufficiently small, for all $\varepsilon \in\left[0, \varepsilon_{0}\right]$

$$
\Phi_{\varepsilon}(X) \cap Y \neq \emptyset
$$


by analyzing the local behavior of intersections. We also have $\Phi_{\varepsilon}(\partial X) \cap$ $Y=\emptyset$, using the compactness of $\partial X$ and the continuous dependence of $\Phi_{\varepsilon}$ on $\varepsilon$.

Clearly (9.4) implies $\Phi: X \times\left[0, \varepsilon_{0}\right] \rightarrow Y$ is transversal to $Z$. So $\Phi^{-1}(Z):=$ $W$ is a differentiable submanifold in $X \times\left[0, \varepsilon_{0}\right]$. Actually it is $\operatorname{in} \operatorname{int}(X) \times$ $\left[0, \varepsilon_{0}\right]$, because $\Phi\left(\partial X \times\left[0, \varepsilon_{0}\right]\right) \cap Z=\emptyset$. So $\partial W \subset X \times\left\{0, \varepsilon_{0}\right\}$.

Note the following elementary fact during the proof of parameterized transversality in [Hir]:

$$
\Phi_{\varepsilon} \text { transversal to } Y \Longleftrightarrow \varepsilon \text { is a regular value of } \pi: W \rightarrow I,
$$

where $\pi: X \times I \rightarrow I$ is the natural projection. Then we have a submersion $\pi: W \rightarrow\left[0, \varepsilon_{0}\right]$ by translating (9.4) via (9.6). By (9.5) $\pi$ is surjective. Picking any metric on $W$, then the gradient vector field $\nabla \pi$ never vanishes on $W$. Let the time- $\tau$ flow of the gradient vector field to be $\varphi_{\pi}^{\tau}$. By Morse theory, we have the diffeomorphism $\varphi_{\pi}^{\varepsilon}: \pi^{-1}(0) \rightarrow \pi^{-1}(\varepsilon)$ for all $\varepsilon \in\left[0, \varepsilon_{0}\right]$, using that $\partial W \subset X \times\left\{0, \varepsilon_{0}\right\}$. Noting that $\pi^{-1}(\varepsilon)=\Phi_{\varepsilon}^{-1}(Z)$, the map $G:=$ $\varphi_{\pi}^{(\cdot)}: \Phi_{0}^{-1}(Z) \times\left[0, \varepsilon_{0}\right] \rightarrow \Phi^{-1}(Z)$ is desired.

First we derive

Corollary 9.1. For given generic $f$ and $J$, there exists a constant $\varepsilon_{0}>$ 0 , such that for all $\left(u_{-}, \chi, u_{+}\right) \in \mathcal{M}^{\varepsilon}\left(\left[z_{-}, w_{-}\right] ; f ;\left[z_{+}, w_{+}\right] ; A_{ \pm}\right)$where $\varepsilon \in$ $\left(0, \varepsilon_{0}\right]$, the linearized operator $E(u)$ in Section 5.1 is surjective.

Proof. In the above lemma, take $X=\overline{\mathcal{M}}_{1}\left(\left[z_{-}, w_{-}\right] ; A_{-}\right) \times \overline{\mathcal{M}}_{1}\left(\left[z_{+}, w_{+}\right] ; A_{+}\right)$, $Y=M \times M, Z=\Delta$ and $\Phi: X \times I \rightarrow Y$ to be

$$
\begin{aligned}
& \phi_{f}^{\varepsilon} e v_{-} \times e v_{+}: \mathcal{M}\left(\left[z_{-}, w_{-}\right] ; A_{-}\right) \times \mathcal{M}\left(\left[z_{+}, w_{+}\right] ; A_{+}\right) \rightarrow M \times M \\
& \quad\left(u_{-}, u_{+}\right) \rightarrow\left(\phi_{f}^{\varepsilon} u_{-}\left(o_{-}\right), u_{+}\left(o_{+}\right)\right),
\end{aligned}
$$

which smoothly extends to $\overline{\mathcal{M}}_{1}\left(\left[z_{-}, w_{-}\right] ; A_{-}\right) \times \overline{\mathcal{M}}_{1}\left(\left[z_{+}, w_{+}\right] ; A_{+}\right)$. Then $X$ is a compact manifold, $\Phi$ is a differentiable map, and $\Phi_{0}=e v_{-} \times e v_{+}$is transversal to $Z=\Delta$ by our assumption on $J^{ \pm}$. Then by the above Lemma 9.2, the condition (9.3) can be achieved for all $\varepsilon \in\left[0, \varepsilon_{0}\right]$.

For given generic $J^{ \pm}$, Lemma 9.1 says $D_{u_{-}} \bar{\partial}_{\left(K_{-}, J_{-}\right)}$and $D_{u_{+}} \bar{\partial}_{\left(K_{-}, J_{-}\right)}$are surjective for $\left(u_{-}, \chi, u_{+}\right) \in \mathcal{M}^{\varepsilon}\left(\left[z_{-}, w_{-}\right] ; f ;\left[z_{+}, w_{+}\right] ; A_{ \pm}\right)$where $\varepsilon \in\left[0, \varepsilon_{0}\right]$. Combining the condition (9.3), by Proposition 5.1, the corollary follows.

Then we prove the central result of this section.

Proposition 9.1. For given generic $f$ and $J$, there exists a constant $\varepsilon_{0}>0$ and a differentiable map

$$
G: \mathcal{M}^{0}\left(\left[z_{-}, w_{-}\right], f,\left[z_{+}, w_{+}\right] ; A_{ \pm}\right) \times\left[0, \varepsilon_{0}\right] \rightarrow \mathcal{M}\left(\left[z_{-}, w_{-}\right], f,\left[z_{+}, w_{+}\right] ; A_{ \pm}\right),
$$


such that for any $\varepsilon \in\left[0, \varepsilon_{0}\right]$,

$G_{\varepsilon}:=G(\cdot, \varepsilon): \mathcal{M}^{0}\left(\left[z_{-}, w_{-}\right], f,\left[z_{+}, w_{+}\right] ; A_{ \pm}\right) \rightarrow \mathcal{M}^{\varepsilon}\left(\left[z_{-}, w_{-}\right] ; f ;\left[z_{+}, w_{+}\right] ; A_{ \pm}\right)$, is a diffeomorphism.

Proof. We take $X, Y, Z$ and $\Phi$ the same as the above corollary. Then all conditions in Lemma 9.2 hold except the condition $\Phi_{0}(\partial X) \cap Z=\emptyset$. We show this condition also holds. Otherwise, we can find $\left(u_{-}, u_{+}\right) \in$ $\partial\left(\overline{\mathcal{M}}_{1}\left(\left[z_{-}, w_{-}\right] ; A_{-}\right) \times \overline{\mathcal{M}}_{1}\left(\left[z_{+}, w_{+}\right] ; A_{+}\right)\right)$, such that $\Phi_{0}\left(\left(u_{-}, u_{+}\right)\right)=\left(e v_{-} \times\right.$ $\left.e v_{+}\right)\left(u_{-}, u_{+}\right) \in \Delta$. In other words, $u_{-}\left(o_{-}\right)=u_{+}\left(o_{+}\right)$, and at least one of $u_{-}$and $u_{+}$is in the compactified space $\overline{\mathcal{M}}_{1}\left(\vec{z}_{ \pm} ; A_{ \pm}\right)$, say $u_{+} \in \overline{\mathcal{M}}_{1}$ $\left.\left(\left[z_{+}, w_{+}\right] ; A_{+}\right)\right)$. Then $u_{+}$must contain some bubble. This is impossible because it contradicts with the rigidity assumption of nodal Floer trajectories, as explained in the proof of Lemma 9.1.

Then we apply part (2) of Lemma 9.2 and get the desired map $G$.

From Proposition 9.1, we see the moduli space

$$
\mathcal{M}^{\text {para }}=\bigcup_{\varepsilon \in\left[0, \varepsilon_{0}\right)} \mathcal{M}^{\varepsilon}\left(\left[z_{-}, w_{-}\right] ; f ;\left[z_{+}, w_{+}\right] ; A_{ \pm}\right)
$$

is a one-dimensional manifold with boundary $\mathcal{M}^{0}\left(\left[z_{-}, w_{-}\right] ; f ;\left[z_{+}, w_{+}\right] ; A_{ \pm}\right)$.

Remark 9.1. There is a slight cheating in the proof of Corollary 9.1 and Proposition 9.1: The $\left.X:=\overline{\mathcal{M}}_{1}\left(\left[z_{+}, w_{+}\right] ; A_{+}\right)\right) \times \overline{\mathcal{M}}_{1}\left(\left[z_{-}, w_{-}\right] ; A_{-}\right)$is not really a compact manifold with boundary. However, for small $\varepsilon_{0}$, we can show for all $\varepsilon \in\left[0, \varepsilon_{0}\right], \Phi_{\varepsilon}(X-\operatorname{int}(X)) \cap \Delta=\emptyset$, this is by the same argument as in Lemma 9.1. Then we can shrink $X$ a bit to $X^{\text {shr }}$, where $X^{\text {shr }}$ is a compact manifold with boundary, and $\Phi_{\varepsilon}\left(X-X^{\text {shr }}\right) \cap \Delta=\emptyset$ for all $\varepsilon \in\left[0, \varepsilon_{0}\right]$. Then we can replace $X$ by $X^{\text {shr }}$ and apply Proposition 9.1.

\section{Smoothing of nodal Floer trajectories II; to Floer trajectories}

In this section, we will carry out the gluing of the perturbed $\boldsymbol{J}$-holomorphic curves $u_{ \pm}$and the local model curve and produce $\varepsilon$-dependent one-parameter family of resolved Floer trajectories.

Let's recall the domains of these curves. The domain of $u_{+}$is a punctured Riemann surfaces $\dot{\Sigma}_{+}$with a puncture $e_{+}$and a marked point $o_{+}$, where $\Sigma_{+} \cong S^{2}$, and $\dot{\Sigma}_{+} \cong \mathbb{C}$. Similarly, for the domain $\dot{\Sigma}_{-}$of $u_{-}$. Let

$$
\begin{array}{ll}
E_{-}=\left\{(\tau, t) \mid(\tau, t) \in(-\infty, 0] \times S^{1}\right\}, & O_{-}=\left\{(\tau, t) \mid(\tau, t) \in[0,+\infty) \times S^{1}\right\}, \\
E_{+}=\left\{(\tau, t) \mid(\tau, t) \in[0,+\infty) \times S^{1}\right\}, & O_{+}=\left\{(\tau, t) \mid(\tau, t) \in(-\infty, 0] \times S^{1}\right\}
\end{array}
$$

be the analytic charts on $\Sigma_{ \pm}$around the punctures $e_{ \pm}$and marked points $o_{ \pm}$, respectively. Then $z=\mathrm{e}^{2 \pi(\tau+\mathrm{i} t)}$ is the given analytic coordinates near $e_{+}$and $o_{-}$, and $z=\mathrm{e}^{-2 \pi(\tau+\mathrm{i} t)}$ is the analytic coordinates near $e_{-}$and $o_{+}$. Note that the $(\tau, t)$ in different charts are different local coordinates, but to 
keep the notation simple we still denote them by the same variables $(\tau, t)$. We have

$$
u_{ \pm}: \dot{\Sigma}_{ \pm} \rightarrow(M, \omega, J), \quad u_{ \pm}\left(o_{ \pm}\right)=p .
$$

We remark that the analytic charts $E_{ \pm}, O_{ \pm}$are unique up to $\tau$-translation and $t$-rotation.

On the $E_{ \pm}$and $O_{ \pm}$we put the metric $g_{(-\infty, 0] \times S^{1}}$ or $g_{[0,+\infty) \times S^{1}}$ in the obvious way. We extend the metric to the remaining part of $\dot{\Sigma}_{ \pm}$in any way, and then fix it.

For the gluing purpose, we need to consider three metrics on the manifold $M$ : the original metric $g$, the Darboux-cylindrical metric $g_{\delta, p}$ and the degenerating metric $g_{\delta, \varepsilon, p}$. The definitions of these metrics are in order:

Let $\delta>0$ be a fixed number less than the injective radius of $(M, g)$. Assume $\delta$ is so small that for every $p$ in $M, B_{\delta}(p)$ is contained in a Darboux neighborhood $U_{p}$ of $p$. Then

$$
\frac{1}{\delta}\left(\exp _{p}^{I}\right)^{-1}: B_{\delta}(p) \rightarrow B_{1}(0) \subset\left(T_{p} M, g_{p}\right) \cong\left(\mathbb{C}^{n}, g_{s t}\right) .
$$

Via the diffeomorphism, $\mathbb{R} \times S^{2 n-1} \cong \mathbb{C}^{n} \backslash\{0\} ;(s, \Theta) \mapsto\left(e^{s} \Theta\right)$, we can pull back the standard metric on $\mathbb{R} \times S^{2 n-1}$ to define the metric on $B_{\delta}(p) \backslash\{p\}$ such that it is isometric to $S^{2 n-1} \times(-\infty, 0]$.

10.1. Construction of approximate solutions. Given any nodal Floer trajectory $\left(u_{-}, u_{+}\right)$, from $(8.6) u_{ \pm}$has the asymptote

$$
\left|\nabla^{k}\left(\Theta_{ \pm}(\tau, t)-\gamma_{ \pm}(t)\right)\right|_{S^{2 n-1}} \leq C_{k} \mathrm{e}^{\frac{-2 \pi c_{k}|\tau|}{p}},
$$

and

$$
\left|\nabla^{k}\left(s \pm(\tau, t)-2 \pi\left(\tau-\tau_{ \pm}\right)\right)\right| \leq C_{k} \mathrm{e}^{\frac{-2 \pi c_{k}|\tau|}{p}},
$$

where $s_{ \pm}=s \circ u_{ \pm}$and $\Theta_{ \pm}=\Theta \circ u_{ \pm}$. Here, the number $p>2$ shouldn't be confused with the point $p$ on $M$.

Let $f: M \rightarrow \mathbb{R}$ be a given Morse function. We choose $f$ so that $\|f\|_{C^{2}}$ is sufficiently small. In particular, we assume

$$
|\nabla f| \leq 1
$$

which can be always achieved by rescaling $f$.

Given the nodal Floer trajectory $\left(u_{-}, u_{+}\right)$with the nodal point $p$, we construct a normalized local model curves $u_{0}$ in the following way:

Lemma 10.1. $u_{0}$ defines a proper map and satisfies

$$
\begin{aligned}
u_{0}: \mathbb{R} \times S^{1} & \rightarrow\left(T_{p} M, J_{p}\right) \cong\left(\mathbb{C}^{n}, J_{s t}\right), \\
(t, \tau) & \rightarrow\left(\Theta_{0}(\tau, t), s_{0}(\tau, t)\right) \in \mathbb{R} \times S^{2 n-1}
\end{aligned}
$$

when $|\tau|$ large, and satisfies the followings:

- $\frac{\partial u_{0}}{\partial \bar{z}}=\nabla f(p)$. 
- In the cylindrical end of $\mathbb{C}^{n}$, it has the same asymptote as $u_{ \pm}$in each of its ends, in the sense that

$$
\begin{aligned}
\mathrm{e}^{\frac{2 \pi \delta|\tau|}{p}}\left|\Theta_{0}(\tau, t)-\gamma_{ \pm}(t)\right|_{S^{2 n-1}} \in W^{1, p}\left(O_{ \pm}\right) \quad \text { and } \\
\mathrm{e}^{\frac{2 \pi \delta|\tau|}{p}}\left|s_{0}(\tau, t)-2 \pi\left( \pm \tau-\tau_{ \pm}\right)\right| \in W^{1, p}\left(O_{ \pm}\right),
\end{aligned}
$$

where

$$
O_{+} \cong(-\infty, 0] \times S^{1}, \quad O_{-} \cong[0,+\infty) \times S^{1}
$$

are the cylindrical charts of the ends $\{ \pm \infty\} \times S^{1}$ in $\mathbb{R} \times S^{1}$ respectively, - $\int_{S^{1}} u_{0}(0, t) d t=0$.

Such $u_{0}$ is unique.

Proof. All the properties are immediate consequences of the expression of the model curves $u(z)=\vec{A} z+\vec{B} / z+\vec{C}+\vec{a} \tau$ given in (6.5). Here in (6.5) we take $\vec{A}=\mathrm{e}^{-2 \pi \tau_{+}} \gamma_{+}(0), \vec{B}=\mathrm{e}^{-2 \pi \tau_{-}} \gamma_{-}(0)$ and $\vec{a}=\nabla f(p)$. We only comment on the last two properties. For the last one, we have only to choose $\vec{C}=0$ in (6.5). On the other hand, for the second property, we use the fact $\tau / \mathrm{e}^{z} \rightarrow 0$ as $\tau \rightarrow \infty$ and so the contribution of $\nabla f(p) \tau$ is negligible compared to $A \mathrm{e}^{2 \pi(\tau+\mathrm{i} t)}+B \mathrm{e}^{-2 \pi(\tau+\mathrm{i} t)}$. (Detailed calculation was carried out in Section 7). For the uniqueness of $u_{0}$, notice that $w_{0}:=u_{0}-\nabla f(p) \tau$ is a holomorphic function from $S^{1} \times \mathbb{R}$ to $\mathbb{C}^{n}$, and on $o_{ \pm} \in D_{ \pm}, w_{0}$ can only have simple pole because the $\Theta$ component of $w_{0}$ converges to simple Reeb orbits $\gamma_{ \pm}(t) \subset S^{2 n-1}$. Therefore, the Laurent series of $w_{0}(z)$ must be $w_{0}(z)=\vec{A} z+\vec{B} / z+\vec{C}$ for some constant vectors $\vec{A}, \vec{B}$ and $\vec{C}$. Since $w_{0}$ has the same asymptote as $u_{0}$, the $\vec{A}, \vec{B}$ and $\vec{C}$ coincides with the ones given in the beginning of the proof.

Remark 10.1. From the above lemma, we see $u_{0}$ can be explicitly given as

$$
u_{0}(\tau, t)=\vec{A} z+\vec{B} / z+\vec{a} \tau,
$$

where $\vec{A}=\mathrm{e}^{-2 \pi \tau_{+}} \gamma_{+}(0), \vec{B}=\mathrm{e}^{-2 \pi \tau_{-}} \gamma_{-}(0), \vec{a}=\nabla f(p)$ and $z=\mathrm{e}^{2 \pi(\tau+i t)}$. We call $u_{0}$ the normalized local model, because $\vec{C}$ has been normalized to zero.

From the expression of $u_{0}$, and the definition of $\vec{A}$ and $\vec{B}$, we get

$$
\left|\nabla^{k}\left(u_{0}(\tau, t)-\mathrm{e}^{2 \pi\left(\tau-\tau_{+}\right)} \gamma_{+}(t)\right)\right| \leq C_{k} \mathrm{e}^{\frac{-2 \pi c_{k}\left(\tau-\tau_{+}\right)}{p}}, \tau>0 .
$$

in the cylindrical metric $|\cdot|$ in $\mathbb{C}^{n}$ for some constants $C_{k}$ and $c_{k}$. Similar result holds for another end of $u_{0}$ when $\tau<0$. Note the convergence (10.5) is stronger than our original requirement (10.3), because $\delta$ is chosen to be smaller than the least $c_{k}$.

In Theorem 5.1, we have proved that for generic $J$, for any nodal Floer trajectory $\left(u_{-}, u_{+}\right)$, the $\left[d u_{-}\left(o_{-}\right)\right]$and $\left[d u_{+}\left(o_{+}\right)\right]$are linearly independent. Consequently, $\vec{A}$ and $\vec{B}$ are linearly independent in $\mathbb{C}^{n}$ for the normalized 
local model $u_{0}$ sitting in $T_{p} M$, where $p=u_{+}\left(o_{+}\right)=u_{-}\left(o_{-}\right)$is the node. From the linear independence of $\vec{A}$ and $\vec{B}$ we get

$$
\min _{t \in S^{1}}\left|u_{0}(0, t)\right| \geq b>0
$$

for some constant $b$.

We consider the scaled local model curve

$$
u_{0}^{\varepsilon}:=\varepsilon u_{0}=\varepsilon(\vec{A} z+\vec{B} / z+\vec{a} \tau) .
$$

From the asymptote (10.5) of $u_{0}$, we derive

$$
\left|\nabla^{k}\left(u_{0}^{\varepsilon}(\tau, t)-\mathrm{e}^{2 \pi\left(\tau-\tau_{+}-2 R(\varepsilon)\right)} \gamma_{+}(t)\right)\right| \leq C_{k} \mathrm{e}^{-2 \pi c_{k}\left(\tau-\tau_{+}-2 R(\varepsilon)\right)}, \tau>0 .
$$

Here

$$
R(\varepsilon)=-\frac{1}{4 \pi} \ln \varepsilon
$$

Similar result holds for another end of $u_{0}^{\varepsilon}$ when $\tau<0$.

Lemma 10.2. Consider the scaled local model curve $u_{0}^{\varepsilon}$ chosen in (10.7). For any given $0<\alpha<2$, there exists $\delta_{\varepsilon}>\varepsilon$ such that

$$
\delta_{\varepsilon} \rightarrow 0, \quad \delta_{\varepsilon} / \varepsilon \rightarrow \infty
$$

and

$$
u_{0}^{\varepsilon}\left([-\alpha R(\varepsilon), \alpha R(\varepsilon)] \times S^{1}\right) \subset B_{p}\left(\delta_{\varepsilon}\right) \subset M .
$$

Proof. We have when $\tau \rightarrow+\infty$,

$$
\left|u_{0}^{\varepsilon}(\alpha R(\varepsilon), t)\right| \sim \varepsilon \cdot|\vec{A}| \cdot \mathrm{e}^{2 \pi \cdot\left(-\alpha \frac{1}{4 \pi} \ln \varepsilon\right)}=|\vec{A}| \varepsilon^{1-\frac{\alpha}{2}} .
$$

Similar result holds for the other end when $\tau \rightarrow-\infty$. So the choice

$$
\delta_{\varepsilon}=\varepsilon^{1-\frac{\alpha}{2}}
$$

will do our purpose.

We choose different cylindrical coordinates near the marked point $o_{ \pm}$of $u_{ \pm}$and get the re-parameterization of the outer curves there (Figure 6)

$$
u_{+}^{\varepsilon}=u_{+}(\tau-2 R(\varepsilon), t) \quad \text { and } \quad u_{-}^{\varepsilon}=u_{-}(\tau+2 R(\varepsilon), t) .
$$

We compare the asymptote of $u_{0}^{\varepsilon}$ and $u_{+}^{\varepsilon}$ for $\tau$ in the range of $[R(\varepsilon)-$ $1, R(\varepsilon)+1]$. It turns out that they get close exponentially as $\varepsilon \rightarrow 0$ : in $[R(\varepsilon)-1, R(\varepsilon)+1]$, by $(10.1)$

$$
\begin{aligned}
& \left|\nabla^{k}\left(u_{+}^{\varepsilon}(\tau, t)-\mathrm{e}^{2 \pi\left(\tau-\tau_{+}-2 R(\varepsilon)\right)} \gamma_{+}(t)\right)\right| \\
& \quad=\left|\nabla^{k}\left(u_{+}(\tau-2 R(\varepsilon), t)-\mathrm{e}^{2 \pi\left(\tau-\tau_{+}-2 R(\varepsilon)\right)} \gamma_{+}(t)\right)\right| \\
& \quad<C_{k} \mathrm{e}^{\frac{-2 \pi c_{k}\left|\tau-\tau_{+}-2 R(\varepsilon)\right|}{p}} .
\end{aligned}
$$




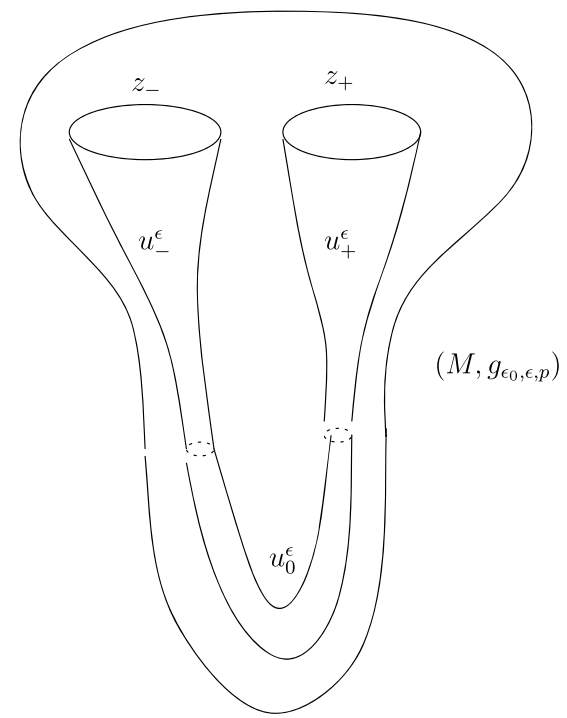

Figure 6. Preglued solution.

Combining (10.8) and (10.10), we see for $\tau \in[R(\varepsilon)-1, R(\varepsilon)+1]$,

$$
\begin{aligned}
\left|\nabla^{k}\left(u_{0}^{\varepsilon}(\tau, t)-u_{+}^{\varepsilon}(\tau, t)\right)\right| & <2 \max _{\tau \in[R(\varepsilon)-1, R(\varepsilon)+1]} C_{k} \mathrm{e}^{\frac{-2 \pi c_{k}\left|\tau-2 R(\varepsilon)-\tau_{+}\right|}{p}} \\
& \leq C_{k} \mathrm{e}^{\frac{-2 \pi c_{k}\left|R(\varepsilon)+1-2 R(\varepsilon)-\tau_{+}\right|}{p}} \\
& =C_{k} \mathrm{e}^{\frac{-2 \pi c_{k}\left|R(\varepsilon)-1+\tau_{+}\right|}{p}} \rightarrow 0
\end{aligned}
$$

as $\varepsilon \rightarrow 0$. Similarly, we can prove the closeness of $u_{0}^{\varepsilon}$ and $u_{-}^{\varepsilon}$ when $\tau$ is in $[-R(\varepsilon)-1,-R(\varepsilon)+1]$.

Next, we construct the approximate solution

$$
u_{\mathrm{app}}^{\varepsilon}(\tau, t)= \begin{cases}u_{-}, & \tau \in \Sigma_{-} \backslash O_{-}, \\ u_{-}^{\varepsilon}(\tau, t), & \tau \in[-2 R(\varepsilon),-R(\varepsilon)-1], \\ \chi_{\varepsilon}(\tau) u_{0}^{\varepsilon}(\tau, t) & \\ \quad+\left(1-\chi_{\varepsilon}(\tau)\right) u_{-}^{\varepsilon}(\tau, t), & \tau \in[-R(\varepsilon)-1,-R(\varepsilon)+1], \\ u_{0}^{\varepsilon}(\tau, t), & \tau \in[-R(\varepsilon)+1, R(\varepsilon)-1], \\ \chi_{\varepsilon}(\tau) u_{0}^{\varepsilon}(\tau, t) & \\ \quad+\left(1-\chi_{\varepsilon}(\tau)\right) u_{+}^{\varepsilon}(\tau, t), & \tau \in[R(\varepsilon)-1, R(\varepsilon)+1], \\ u_{+}^{\varepsilon}(\tau, t), & \tau \in[R(\varepsilon)+1,2 R(\varepsilon)], \\ u_{+}, & \tau \in \Sigma_{+} \backslash O_{+},\end{cases}
$$


where the cut-off function $\chi_{\varepsilon}: \mathbb{R} \rightarrow[0,1]$ satisfies

$$
\begin{gathered}
\chi_{\varepsilon}(\tau)= \begin{cases}1, & \text { for }|\tau| \leq R(\varepsilon)-1, \\
0, & \text { for }|\tau| \geq R(\varepsilon)+1,\end{cases} \\
\left|\chi_{\varepsilon}^{\prime}(\tau)\right| \leq 1 .
\end{gathered}
$$

In the above formula, the summation "+" is with respect to the linear space structure of $T_{p} M$ (by the Darboux cylindrical chart, we can think the local model lies in $\left.T_{p} M\right)$.

Now by applying a version of the implicit function theorem or the Newton's iteration method, we want to perturb $u_{\text {app }}^{\varepsilon}$ to a genuine solution $u^{\varepsilon}$ of the resolved Floer trajectory equation

$$
\bar{\partial}_{J} u^{\varepsilon}+\left(P_{\varepsilon \chi_{\varepsilon}(\tau) f}\left(u^{\varepsilon}\right)\right)_{J}^{(0,1)}=0,
$$

where

$$
\begin{aligned}
P_{\varepsilon \chi_{\varepsilon}(\tau) f}\left(u^{\varepsilon}\right) & =\varepsilon \chi_{\varepsilon}(\tau)\left(J X_{f}\left(u^{\varepsilon}\right) d \tau-X_{f}\left(u^{\varepsilon}\right) d t\right) \\
& =\varepsilon \chi_{\varepsilon}(\tau)\left(\nabla f\left(u^{\varepsilon}\right) d \tau-J \nabla f\left(u^{\varepsilon}\right) d t\right) .
\end{aligned}
$$

For the simplicity of notations, we write

$$
a^{\varepsilon}=P_{\varepsilon \chi_{\varepsilon}(\tau) f}\left(u^{\varepsilon}\right)
$$

and then

$$
a^{\varepsilon}\left(\frac{\partial}{\partial \tau}\right)=\varepsilon \chi_{\varepsilon}(\tau) \nabla f\left(u^{\varepsilon}\right) .
$$

In the conformal coordinates $(\tau, t)$ and the cylindrical metric, we have the identity

$$
\left|a^{\varepsilon}\right|^{2}=2\left|a^{\varepsilon}\left(\frac{\partial}{\partial \tau}\right)\right|^{2}
$$

and so it will be enough to estimate the latter norm. Therefore, we will carry out estimation of this latter norm below.

10.2. Error estimates of approximate solutions. With the choice of metric $g_{\varepsilon_{0}, \varepsilon, p}$ in the beginning of this section, we carry out the error estimates, i.e., the point estimate and $L^{p}$ estimate for the norm

$$
\left|\bar{\partial}_{J} u_{\text {app }}^{\varepsilon}-\left(P_{K_{R(\varepsilon)}}\left(u_{\text {app }}^{\varepsilon}\right)\right)_{J}^{(0,1)}\right|_{g_{\varepsilon_{0}, \varepsilon, p}} .
$$

Convention: In many estimates of this subsection, there are different constants $C$ 's. The exact values are not important; The importance is that all of them are independent on $\varepsilon$. For this reason, we just denote them by the same symbol $C$ and shouldn't cause problems.

We split this estimation into three regions:

(1) the region for $|\tau| \leq \frac{2}{3} R(\varepsilon)$,

(2) the region for $\frac{2}{3} R(\varepsilon) \leq|\tau| \leq R(\varepsilon)+1$,

(3) the region for $R(\varepsilon)+1 \leq|\bar{\tau}| \leq 2 R(\varepsilon)$. 
Case 1: For $|\tau| \leq \frac{2}{3} R(\varepsilon), u_{\text {app }}^{\varepsilon}=u_{0}^{\varepsilon}$. Recall $R(\varepsilon)=-\frac{1}{4 \pi} \ln \varepsilon$. By taking $\alpha=\frac{2}{3}$ in Lemma 10.2, we have

$$
u_{0}^{\varepsilon}\left(\left[-\frac{2}{3} R(\varepsilon), \frac{2}{3} R(\varepsilon)\right] \times S^{1}\right) \subset B_{\delta_{\varepsilon}}(p),
$$

where $\delta_{\varepsilon}=\varepsilon^{\frac{2}{3}}$. The local model $u_{0}^{\varepsilon} \subset\left(T_{p} M, J_{p}\right) \cong \mathbb{C}^{n}$ satisfies $\bar{\partial}_{J_{p}} u_{0}^{\varepsilon}-$ $\varepsilon \nabla f(p)=0$. Therefore,

$$
\begin{aligned}
\bar{\partial}_{J} u_{\mathrm{app}}^{\varepsilon}-a^{\varepsilon} & =\left(\bar{\partial}_{J} u_{0}^{\varepsilon}-a^{\varepsilon}\right)-\left(\bar{\partial}_{J_{p}} u_{0}^{\varepsilon}-\varepsilon \nabla f(p)\right) \\
& =\left(\bar{\partial}_{J} u_{0}^{\varepsilon}-\bar{\partial}_{J_{p}} u_{0}^{\varepsilon}\right)-\varepsilon\left(\chi_{\varepsilon}(\tau) \nabla f\left(u_{0}^{\varepsilon}\right)-\nabla f(p)\right) \\
& =\frac{1}{2}\left(J-J_{p}\right) d u_{0}^{\varepsilon} \circ \mathrm{i}-\varepsilon\left(\nabla f\left(u_{0}^{\varepsilon}\right)-\nabla f(p)\right) .
\end{aligned}
$$

We have

$$
\begin{aligned}
\left\|J(x)-J_{p}\right\| & \leq C\|D J(p)\|_{B_{\delta_{\varepsilon}}(p)} \cdot|x|_{g}, \\
|\nabla f(x)-\nabla f(p)| & \leq C\left\|D^{2} f\right\|_{B_{\delta_{\varepsilon}}(p)} \cdot|x|_{g},
\end{aligned}
$$

where $|x|_{g}$ is the Euclidean norm $g(p)$ in the Darboux chart at $p$.

On the other hand for the normalized local model $u_{0}^{\varepsilon}$ with $z=\mathrm{e}^{2 \pi(\tau+\mathrm{i} t)}$, we have

$$
\left|\frac{\partial u_{0}^{\varepsilon}}{\partial \tau}\right|_{g},\left|\frac{\partial u_{0}^{\varepsilon}}{\partial t}\right|_{g} \sim\left|u_{0}^{\varepsilon}\right|_{g} \leq C \delta_{\varepsilon}
$$

Therefore,

$$
\left|d u_{0}^{\varepsilon}\right|_{g} \leq C \delta_{\varepsilon} .
$$

Since $u_{0}^{\varepsilon}\left(\left[-\frac{2}{3} R(\varepsilon), \frac{2}{3} R(\varepsilon)\right] \times S^{1}\right) \subset B_{\delta_{\varepsilon}}(p)$, on the image of $u_{0}^{\varepsilon}$, the almost complex structure deviates from the standard complex structure $J_{p}$ on $T_{p} M$ by

$$
\left\|J\left(u_{0}^{\varepsilon}\right)-J_{p}\right\| \leq C\left\|D J\left(u_{0}^{\varepsilon}\right)\right\| \cdot\left|u_{0}^{\varepsilon}\right|_{g}
$$

where $\|\cdot\|$ is the operator norm of linear maps $L: V \rightarrow V$. We emphasize that the norm $\|L\|$ is independent on the conformal class of constant metrics on $V$. Therefore, (10.21) holds regardless of our choice of metrics $g$ or $g_{\delta, \varepsilon, p}$.

On the other hand, we obtain

$$
\varepsilon\left|\nabla f\left(u_{0}^{\varepsilon}\right)-\nabla f(p)\right|_{g} \leq C \varepsilon\left|u_{0}^{\varepsilon}\right|_{g} .
$$

Now we are ready to estimate $\left|\bar{\partial}_{J} u_{\text {app }}^{\varepsilon}-a^{\varepsilon}\right|_{g_{\varepsilon_{0}, \varepsilon, p}}$. By (10.16), (10.21) and (10.20),

$$
\begin{aligned}
\left|\bar{\partial}_{J} u_{\mathrm{app}}^{\varepsilon}-a^{\varepsilon}\right|_{g} & \leq \frac{1}{2}\left\|J-J_{0}\right\|\left|d u_{0}^{\varepsilon}\right|_{g}+\varepsilon\left|\nabla f\left(u_{0}^{\varepsilon}\right)-\nabla f(p)\right|_{g} \\
& \leq C\left(\left|u_{0}^{\varepsilon}\right|_{g}\left|d u_{0}^{\varepsilon}\right|_{g}+\varepsilon\left|u_{0}^{\varepsilon}\right|_{g}\right) \\
& \leq C\left(\delta_{\varepsilon}^{2}+\varepsilon \delta_{\varepsilon}\right) .
\end{aligned}
$$


Since $u_{0}^{\varepsilon}(\tau, t) \in B_{\delta_{\varepsilon}}(p)$, and noting in $B_{\delta_{\varepsilon}}(p)$ the metric $g_{\varepsilon_{0}, \varepsilon, p} \leq \frac{1}{\varepsilon^{2}} g$, by (10.23), we have

$$
\left|\bar{\partial}_{J} u_{\text {app }}^{\varepsilon}-a^{\varepsilon}\right|_{g_{\varepsilon_{0}, \varepsilon, p}} \leq \frac{1}{\varepsilon}\left|\bar{\partial}_{J} u_{\text {app }}^{\varepsilon}-a^{\varepsilon}\right|_{g} \leq C\left(\delta_{\varepsilon}^{2} / \varepsilon+\delta_{\varepsilon}\right) .
$$

Since $\delta_{\varepsilon}=\varepsilon^{\frac{2}{3}}$, we obtain

$$
\left|\bar{\partial}_{J} u_{\mathrm{app}}^{\varepsilon}-a^{\varepsilon}\right|_{g_{\varepsilon_{0}, \varepsilon, p}} \leq C\left(\varepsilon^{\frac{4}{3}-1}+\varepsilon^{\frac{2}{3}}\right) \leq C \varepsilon^{\frac{1}{3}} .
$$

This error converges to 0 as $\varepsilon \rightarrow 0$.

Case 2: For $\frac{2}{3} R(\varepsilon) \leq|\tau| \leq R(\varepsilon)+1$, by Lemma 10.2 again we have $u_{0}^{\varepsilon}(\tau, t) \in$ $B_{\delta_{\varepsilon}}(p)$, where $\delta_{\varepsilon}=C \varepsilon^{\frac{1}{2}}$. On the other hand,

$$
\left|u_{0}^{\varepsilon}(\tau, t)\right|_{g} \geq \varepsilon \cdot \min \{|\vec{A}|,|\vec{B}|\} \cdot \mathrm{e}^{2 \pi \cdot \frac{2}{3} R(\varepsilon)}=\beta \varepsilon^{\frac{2}{3}},
$$

when $\varepsilon$ is small. So the image of $u_{0}^{\varepsilon}(\tau, t)$ is contained in

$$
B_{\delta_{\varepsilon}}(p) \backslash B_{\beta \varepsilon^{\frac{2}{3}}}(p),
$$

where the metric $g_{\varepsilon_{0}, \varepsilon, p}$ is cylindrical and so $g_{\varepsilon_{0}, \varepsilon, p}(x)=\frac{1}{|x|_{g}^{2}} g(x)$. Therefore,

$$
\left|\bar{\partial}_{J} u_{0}^{\varepsilon}-a^{\varepsilon}\right|_{\varepsilon_{0}, \varepsilon, p}=\frac{1}{\left|u_{0}^{\varepsilon}(\tau, t)\right|_{g}}\left|\bar{\partial}_{J} u_{0}^{\varepsilon}-a^{\varepsilon}\right|_{g} .
$$

Similar to the second inequality in (10.23), we have

$$
\begin{aligned}
\left|\bar{\partial}_{J} u_{\mathrm{app}}^{\varepsilon}-a^{\varepsilon}\right|_{g} & \leq \frac{1}{2}\left\|J-J_{0}\right\|\left|d u_{0}^{\varepsilon}\right|_{g}+\varepsilon\left|\chi_{\varepsilon} \nabla f\left(u_{0}^{\varepsilon}\right)-\nabla f(p)\right|_{g} \\
& \leq C\left(\left|u_{0}^{\varepsilon}\right|_{g}\left|d u_{0}^{\varepsilon}\right|_{g}+\varepsilon\right) .
\end{aligned}
$$

Combining (10.26),(10.27) and (10.28) we get

$$
\left|\bar{\partial}_{J} u_{0}^{\varepsilon}-a^{\varepsilon}\right|_{g_{\varepsilon_{0}, \varepsilon, p}} \leq C\left(\left|d u_{0}^{\varepsilon}\right|_{g}+\frac{\varepsilon}{\beta \varepsilon^{\frac{2}{3}}}\right) \leq C\left(\delta_{\varepsilon}+\varepsilon^{\frac{1}{3}}\right) \leq C^{\prime} \varepsilon^{\frac{1}{3}} .
$$

For $\left|u_{0}^{\varepsilon}(\tau, t)-u_{ \pm}^{\varepsilon}(\tau, t)\right|_{g_{\varepsilon_{0}, \varepsilon, p}}$, since the metric $g_{\varepsilon_{0}, \varepsilon, p}$ is cylindrical in this part, we also have from (10.11) (which is in cylindrical metric)

$$
\left|u_{0}^{\varepsilon}(\tau, t)-u_{ \pm}^{\varepsilon}(\tau, t)\right|_{g_{\varepsilon_{0}, \varepsilon, p}} \leq C \mathrm{e}^{\frac{-2 \pi c_{0} R(\varepsilon)}{p}}=C \varepsilon^{\frac{c_{0}}{2 p}} .
$$


Combining the above (10.29) and (10.30), with respect to the metric $g_{\varepsilon_{0}, \varepsilon, p}$,

$$
\begin{aligned}
\mid \bar{\partial}_{J} & u_{\mathrm{app}}^{\varepsilon}-\left.a^{\varepsilon}\right|_{g_{\varepsilon_{0}, \varepsilon, p}} \\
= & \left|\bar{\partial}_{J}\left(\chi_{\varepsilon}(\tau) u_{0}^{\varepsilon}+\left(1-\chi_{\varepsilon}(\tau)\right) u_{ \pm}\right)-a^{\varepsilon}\right|_{g_{\varepsilon_{0}, \varepsilon, p}} \\
\leq & \chi_{\varepsilon}(\tau)\left|\bar{\partial}_{J} u_{0}^{\varepsilon}-a^{\varepsilon}\right|_{g_{\varepsilon_{0}, \varepsilon, p}}+\left(1-\chi_{\varepsilon}(\tau)\right)\left(\left|\bar{\partial}_{J} u_{ \pm}^{\varepsilon}\right|_{g_{\varepsilon_{0}, \varepsilon, p}}+\left|a^{\varepsilon}\right|_{g_{\varepsilon_{0}, \varepsilon, p}}\right) \\
& +\chi_{\varepsilon}^{\prime}(\tau)\left|u_{0}^{\varepsilon}-u_{ \pm}^{\varepsilon}\right|_{g_{\varepsilon_{0}, \varepsilon, p}} \\
\leq & 1 \cdot C^{\prime} \varepsilon^{\frac{1}{3}}+1 \cdot\left(0+\frac{\varepsilon}{\beta \varepsilon^{\frac{2}{3}}} \cdot|\nabla f|_{g}\right)+1 \cdot C \varepsilon^{\frac{c_{0}}{2 p}}, \\
\leq & C \varepsilon^{\min \left\{\frac{1}{3}, \frac{c_{0}}{2 p}\right\}}
\end{aligned}
$$

In the second of the above inequalities, we have used that $u_{ \pm}^{\varepsilon}=u_{+}(\tau-$ $2 R(\varepsilon), t)$ is $\boldsymbol{J}$-holomorphic, $\left|a^{\varepsilon}\right|_{g_{\varepsilon_{0}, \varepsilon, p}} \leq \frac{1}{\beta \varepsilon^{\frac{2}{3}}}\left|a^{\varepsilon}\right|_{g}$, and $\left|\chi^{\prime}(\tau)\right| \leq 1$.

Case 3: For $R(\varepsilon)+1 \leq|\tau| \leq 2 R(\varepsilon), u_{\text {app }}^{\varepsilon}=u_{ \pm}^{\varepsilon}$ are $\boldsymbol{J}$-holomorphic, and $a^{\varepsilon}=0$, so

$$
\bar{\partial}_{J} u_{\text {app }}^{\varepsilon}-a^{\varepsilon} \equiv 0
$$

In all, we have obtained the point estimate for any $(\tau, t) \in[-2 R(\varepsilon), 2 R(\varepsilon)] \times$ $S^{1}$ :

$$
\operatorname{Err}(\varepsilon):=\left|\bar{\partial}_{J} u_{\text {app }}^{\varepsilon}-a^{\varepsilon}\right|_{g_{\varepsilon_{0}, \varepsilon, p}} \leq C \varepsilon^{\frac{1}{3}} .
$$

The $L_{\alpha_{\delta, \varepsilon}}^{p}$ estimate (The weight $\alpha_{\delta, \varepsilon}$ is defined in the next section): Note that on $\dot{\Sigma}_{ \pm} \backslash O_{ \pm}$the $u_{\text {app }}^{\varepsilon}$ coincides with the two original solutions $u_{+}$and $u_{-}$, so we only need to integrate $\left|\bar{\partial}_{J} u_{\text {app }}^{\varepsilon}-a^{\varepsilon}\right|_{g_{\varepsilon_{0}, \varepsilon, p}}^{p}$ over $[-2 R(\varepsilon), 2 R(\varepsilon)] \times S^{1}$. Note in such region the weight function

$$
\left|\rho_{\varepsilon}(\tau)\right| \leq \mathrm{e}^{2 \pi \delta \cdot(2 R(\varepsilon))}=\varepsilon^{-\delta} .
$$

Therefore, we get

$$
\begin{gathered}
\left\|\bar{\partial}_{J} u_{\text {app }}^{\varepsilon}-a^{\varepsilon}\right\|_{\alpha_{\delta, \varepsilon}}^{p} \leq(\operatorname{Err}(\varepsilon))^{p} \cdot \varepsilon^{-\delta} \cdot 4 R(\varepsilon)=-C \varepsilon^{\min \left\{\frac{p}{3}, \frac{c_{0}}{2}\right\}-\delta} \ln \varepsilon \\
\text { i.e. }\left\|\bar{\partial}_{\left(J_{\varepsilon}, K_{\varepsilon}\right)} u_{\text {app }}^{\varepsilon}\right\|_{p, \alpha_{\delta, \varepsilon}} \leq L \cdot(R(\varepsilon))^{\frac{1}{p}} \cdot \mathrm{e}^{-\frac{4 \pi a R(\varepsilon)}{p}}
\end{gathered}
$$

where $L$ and $a$ are constant independent on $\varepsilon$, and $a=\min \left\{\frac{1}{3}, \frac{c_{0}}{2 p}\right\}-\frac{\delta}{p}$.

If we choose $0<\delta<\min \left\{\frac{p}{3}, \frac{c_{0}}{2}\right\}$ in the beginning, then $a>0$ and so

$$
\left\|\bar{\partial}_{\left(J_{\varepsilon}, K_{\varepsilon}\right)} u_{\mathrm{app}}^{\varepsilon}\right\|_{p, \alpha_{\delta, \varepsilon}} \rightarrow 0
$$

as $\varepsilon \rightarrow 0$, the error estimate is established. For gluing purpose in later sections, we further assume $\delta$ is small in the beginning such that $0<\delta<a$. 
10.3. The off-shell setting of resolved nodal Floer trajectories. We define the Banach manifold to host all resolved nodal Floer trajectories near the enhanced nodal Floer trajectories $u=\left(u_{-}, u_{0}, u_{+}\right)$. The construction is some "smoothing" of the Banach manifold for enhanced nodal Floer trajectories in Section 8.3. Roughly speaking, We smooth the target $(M \backslash\{p\}) \sqcup T_{p} M$ to $\left(M, g_{\varepsilon_{0}, \varepsilon, p}\right)$, smooth the exponential weight for the outer curves and local model to the exponential weight for the approximate solution, and smooth the Morse-Bott movements for the outer curves and local model near their ends. The precise description is in order:

First we define the Banach manifold $\mathcal{B}_{\text {res }}^{\varepsilon}\left(z_{-}, z_{+} ; p\right)$ for any $\varepsilon \in\left(0, \delta_{0}\right)$ and $p \in M$, where $\delta_{0}>0$ is a small constant to be determine later. $\mathcal{B}_{\text {res }}^{\varepsilon}\left(z_{-}, z_{+} ; p\right)$ consists of maps $u$ from $\dot{\Sigma}$ to the Riemannian manifold $\left(M, g_{\varepsilon_{0}, \varepsilon, p}\right)$ satisfying:

(1) $u \in W_{\mathrm{loc}}^{1, p}(\dot{\Sigma}, M)$.

(2) $\lim _{\tau \rightarrow+\infty} u(\tau, t)=z_{+}(t)$ and $\lim _{\tau \rightarrow-\infty} u(\tau, t)=z_{-}(t)$ for all $t \in S^{1}$.

(3) When $\tau>0$ is large enough, $u(\tau, t)=\exp _{z_{+}(t)} \xi(\tau, t)$ for $\xi(\tau, t) \in$ $W_{\delta}^{1, p}\left([0, \infty) \times S^{1}, z_{+}^{*}(T M)\right)$. Similarly, for the other end converging to $z_{-}(t)$.

(4) For each $u \in \mathcal{B}_{\text {res }}^{\varepsilon}\left(z_{-}, z_{+} ; p\right)$, its tangent space $T_{u} \mathcal{B}_{\text {res }}^{\varepsilon}\left(z_{-}, z_{+} ; p\right)$ is identified as $W_{\alpha_{\delta, \varepsilon}}^{1, p}\left(u^{*}(T M)\right)$, defined as the following: for $V$ be a section of $u^{*}(T M)$, we define the $W_{\alpha_{\delta, \varepsilon}}^{1, p}$ norm of $V$ to be

$$
\begin{aligned}
\|V\|_{1, p, \alpha_{\delta, \varepsilon}}^{p}= & |V(-R(\varepsilon), 0)|^{p}+|V(R(\varepsilon), 0)|^{p} \\
& +\int_{[-2 R(\varepsilon), 2 R(\varepsilon)] \times S^{1}} \alpha_{\delta, \varepsilon}(\tau)\left(\left|V-V_{0}\right|^{p}+\left|\nabla\left(V-V_{0}\right)\right|^{p}\right) d \tau d t \\
& +\int_{|\tau|>2 R(\varepsilon)}\left(\left|V-V_{0}\right|^{p}+\left|\nabla\left(V-V_{0}\right)\right|^{p}\right) d \tau d t,
\end{aligned}
$$

where in the above identity, all metric $|\cdot|$ are the metric $g_{\varepsilon_{0}, \varepsilon, p}$, and

$$
\begin{aligned}
V_{0}(\tau, t)= & \beta_{\varepsilon}^{-}(\tau) \operatorname{Pal}_{u(\tau, t)} \operatorname{Pal}_{u(-R(\varepsilon), t)}(V(-R(\varepsilon), 0)) \\
& +\beta_{\varepsilon}^{+}(\tau) \operatorname{Pal}_{u(\tau, t)} \operatorname{Pal}_{u(R(\varepsilon), t)}(V(R(\varepsilon), 0)),
\end{aligned}
$$

where in the above expression $\operatorname{Pal}_{u(\tau, t)}\left(V\left(\tau^{\prime}, t^{\prime}\right)\right)$ is the parallel transport of $V\left(\tau^{\prime}, t^{\prime}\right)$ along the minimal geodesic of the metric $g_{\varepsilon_{0}, \varepsilon, p}$ from $u\left(\tau^{\prime}, t^{\prime}\right)$ to $u(\tau, t)$. The cut-off function $\beta_{\varepsilon}^{ \pm}: \mathbb{R} \rightarrow[0,1]$ is smooth, $0 \leq\left|\frac{d}{d \tau} \beta_{\varepsilon}^{ \pm}\right| \leq 1$

$$
\begin{aligned}
& \beta_{\varepsilon}^{-}(\tau)= \begin{cases}1, & \text { for } 2 \leq \tau \leq 2 R(\varepsilon)-2, \\
0, & \text { for } \tau \leq 1 \text { or } \tau \geq 2 R(\varepsilon)-1,\end{cases} \\
& \beta_{\varepsilon}^{+}(\tau)= \begin{cases}1, & \text { for }-2 R(\varepsilon)+2 \leq \tau \leq-2, \\
0, & \text { for } \tau \geq-1 \text { or } \tau \leq-2 R(\varepsilon)+1 .\end{cases}
\end{aligned}
$$


The weight function $\alpha_{\delta, \varepsilon}$ is smooth,

$$
\alpha_{\delta, \varepsilon}(\tau)= \begin{cases}\mathrm{e}^{2 \pi \delta|\tau|}, & \text { for }|\tau| \leq R(\varepsilon)-1, \\ \sim \mathrm{e}^{\pi \delta R(\varepsilon)}, & \text { for }|\tau| \in[R(\varepsilon)-1, R(\varepsilon)+1], \\ \mathrm{e}^{2 \pi \delta|\tau-2 R(\varepsilon)|}, & \text { for }|\tau| \in[R(\varepsilon)+1,2 R(\varepsilon)], \\ 1, & \text { for }|\tau| \geq 2 R(\varepsilon) .\end{cases}
$$

In the above the " $\sim$ " means that the ratio of $\alpha_{\delta, \varepsilon}(\tau)$ and $e^{\pi \delta R(\varepsilon)}$ is between $\frac{1}{2}$ and $\frac{3}{2}$ for $|\tau| \in[R(\varepsilon)-1, R(\varepsilon)+1]$.

Remark 10.2 (About the "Morse-Bott" variation). The vector field $V_{0}$ is induced from the "Morse-Bott" variation $V( \pm R(\varepsilon), 0)$, which is the approximation of the true Morse-Bott variation of the asymptotes $\left(\tau_{ \pm}, \gamma_{ \pm}\right)$at infinity of the enhanced nodal Floer trajectories $\left(u_{-}, u_{0}, u_{+}\right)$. Given $\left(\tau^{\prime}, t^{\prime}\right)$, there may be different minimal geodesics connecting the points $u(\tau, t)$ and $u\left(\tau^{\prime}, t^{\prime}\right)$ so the symbol $\mathrm{Pal}_{u(\tau, t)}$ is ambiguous, but $\operatorname{such}(\tau, t)$ form at most 1 dimensional subset in $\mathbb{R} \times S^{1}$ so won't affect the $\|\cdot\|_{1, p, \alpha_{\delta, \varepsilon}}$ norm.

Remark 10.3 (About the cut-off function $\beta_{\varepsilon}^{ \pm}$). Recall for the vector field on $u_{+}$, we take out the $\boldsymbol{J}$-holomorphic vector field induced from the MorseBott move when $\tau<-2$, and then measure the remaining part by $W_{\delta}^{1, p}$ norm. Similarly for the vector field on any local model, we take out the $\boldsymbol{J}$ holomorphic vector field induced from the Morse-Bott move when $|\tau|>2$ and then measure the remaining part by $W_{\delta}^{1, p}$ norm. Since $u_{ \pm}^{\varepsilon}=u_{ \pm}(\cdot-$ $\pm 2 R(\varepsilon)$ ), the $\boldsymbol{J}$-holomorphic vector field induced from the Morse-Bott move $V( \pm R(\varepsilon))$ on the approximate solution is taken out when $2 \leq|\tau| \leq 2 R(\varepsilon)-2$. That is why we design the above cut-off function $\beta_{\varepsilon}^{ \pm}$.

Remark 10.4 (About the exponential weight function $\alpha_{\delta, \varepsilon}(\tau)$ ). Since we will use the bound of the right inverses of $D_{u_{ \pm}^{\varepsilon}} \bar{\partial}_{\left(J^{ \pm}, K^{ \pm}\right)}$and $D_{u_{0}^{\varepsilon}} \bar{\partial}_{\left(J_{p}, a^{\varepsilon}\right)}$ to estimate the bound of the right inverse of $D_{u_{\mathrm{app}}^{\varepsilon}} \bar{\partial}_{\left(J^{ \pm}, K^{ \pm}, \varepsilon f\right)}$, the weight function $\alpha_{\delta, \varepsilon}(\tau)$ has to be the concatenation of the weight functions for $u_{ \pm}^{\varepsilon}$ and $u_{0}^{\varepsilon}$. Also the ratio between $\alpha_{\delta, \varepsilon}(\tau)$ and them must be uniformly bounded up and below. That is why we give the above expression for $\alpha_{\delta, \varepsilon}(\tau)$.

Therefore, we have an $\varepsilon$-family of Banach manifolds $\mathcal{B}_{\text {res }}^{\varepsilon}\left(z_{-}, z_{+} ; p\right)$, and an $\varepsilon$-family of equations $\bar{\partial}_{\left(J_{\varepsilon}, K_{\varepsilon}, \varepsilon f\right)} u^{\varepsilon}=0$ defined on each Banach bundle

$$
\pi: \mathcal{L}_{\text {res }}^{\varepsilon}\left(z_{-}, z_{+} ; p\right) \rightarrow \mathcal{B}_{\text {res }}^{\varepsilon}\left(z_{-}, z_{+} ; p\right)
$$

where

$$
\mathcal{L}_{\text {res }}^{\varepsilon}\left(z_{-}, z_{+} ; p\right)=\bigcup_{u \in \mathcal{B}_{\text {res }}^{\varepsilon}\left(z_{-}, z_{+} ; p\right)} L_{\alpha_{\delta, \varepsilon}}^{p}\left(\Lambda^{0,1}\left(u^{*} T M \otimes\right)\right)
$$


and each fiber $L_{\alpha_{\delta, \varepsilon}}^{p}\left(\Lambda^{0,1}\left(u^{*} T M \otimes\right)\right)$ consists of sections $V$ of $\Lambda^{0,1}\left(u^{*} T M \otimes\right)$ such that

$$
\|V\|_{p, \alpha_{\delta, \varepsilon}}=\int_{|\tau| \geq 2 R(\varepsilon)}|V|^{p} d \tau d t+\int_{|\tau| \leq 2 R(\varepsilon)} \alpha_{\delta, \varepsilon}(\tau)|V|^{p} d \tau d t
$$

where the norm $|\cdot|$ is in terms of the metric $g_{\varepsilon_{0}, \varepsilon, p}$.

We define

$$
\mathcal{B}_{\text {res }}^{\varepsilon}\left(z_{-}, z_{+}\right):=\bigcup_{p \in M} \mathcal{B}_{\text {res }}^{\varepsilon}\left(z_{-}, z_{+} ; p\right) .
$$

For $u \in \mathcal{B}_{\text {res }}^{\varepsilon}\left(z_{-}, z_{+}\right)$, its tangent space consists of elements $U=(V, v)$ where $V \in T_{u} \mathcal{B}_{\text {res }}^{\varepsilon}\left(z_{-}, z_{+} ; p\right)$ and $v \in T_{p} M$, with the norm

$$
\|U\|_{1, p, \alpha_{\delta, \varepsilon}}=\|V\|_{1, p, \alpha_{\delta, \varepsilon}}+|v| .
$$

Here $v$ represents the variation of the target Riemannian manifolds $\left(M, g_{\varepsilon_{0}, \varepsilon, p}\right)$, which are parameterized by $M$.

Remark 10.5. The trivialization of the family of Riemannian manifolds

$$
\bigcup_{p \in M}\left(M, g_{\varepsilon_{0}, \varepsilon, p}\right)
$$

is to regard them as pointed manifolds $(M, p)$ and use the trivialization given in Subsection 8.1.

Let

$$
\mathcal{L}_{\text {res }}^{\varepsilon}\left(z_{-}, z_{+}\right):=\bigcup_{p \in M} \mathcal{L}_{\text {res }}^{\varepsilon}\left(z_{-}, z_{+} ; p\right)
$$

then we have a natural section

$$
\bar{\partial}_{\left(J_{\varepsilon}, K_{\varepsilon}, \varepsilon f\right)}: \mathcal{B}_{\text {res }}^{\varepsilon}\left(z_{-}, z_{+}\right) \rightarrow \mathcal{L}_{\text {res }}^{\varepsilon}\left(z_{-}, z_{+}\right) .
$$

The linearization of $\bar{\partial}_{\left(J_{\varepsilon}, K_{\varepsilon}\right)}$ at $u_{\mathrm{app}}^{\varepsilon}$

$$
D \bar{\partial}_{\left(J_{\varepsilon}, K_{\varepsilon}, \varepsilon f\right)}\left(u_{\mathrm{app}}^{\varepsilon}\right): W_{\alpha_{\delta, \varepsilon}}^{1, p}\left(u^{*} T M\right) \rightarrow L_{\alpha_{\delta, \varepsilon}}^{p}\left(\Lambda^{0,1}\left(u^{*} T M \otimes\right)\right)
$$

is given by

$$
D \bar{\partial}_{\left(J_{\varepsilon}, K_{\varepsilon}, \varepsilon f\right)}\left(u_{\mathrm{app}}^{\varepsilon}\right)=D_{u} \bar{\partial}_{J}\left(u_{\mathrm{app}}^{\varepsilon}\right)+D P_{K_{\varepsilon}}\left(u_{\mathrm{app}}^{\varepsilon}\right) .
$$

The linearization of $D \bar{\partial}_{\left(J_{\varepsilon}, K_{\varepsilon}, \varepsilon f\right)}\left(u_{\mathrm{app}}^{\varepsilon}\right)$ with respect to $v$ is similar to that given in Subsections 8.2 and 8.3. 
10.4. Construction and estimates of the right inverse. Given the approximate solution $u_{\mathrm{app}}^{\varepsilon}$, we will construct the approximate right inverse

$$
Q^{\varepsilon}: L_{\alpha_{\delta, \varepsilon}}^{p}\left(\Lambda^{0,1}\left(u_{\mathrm{app}}^{\varepsilon}\right)^{*} T M\right) \rightarrow W_{\alpha_{\delta, \varepsilon}}^{1, p}\left(\left(u_{\mathrm{app}}^{\varepsilon}\right)^{*} T M\right) \oplus T_{p} M
$$

of the differential operator

$$
D_{u} \bar{\partial}_{\left(J_{\varepsilon}, K_{\varepsilon}\right)}\left(u_{\text {app }}^{\varepsilon}\right): W_{\alpha_{\delta, \varepsilon}}^{1, p}\left(\left(u_{\text {app }}^{\varepsilon}\right)^{*} T M\right) \oplus T_{p} M \rightarrow L_{\alpha_{\delta, \varepsilon}}^{p}\left(\Lambda^{0,1}\left(u_{\text {app }}^{\varepsilon}\right)^{*} T M\right),
$$

and show $Q^{\varepsilon}$ is uniformly bounded in operator norm. For notation brevity, we write $D_{u} \bar{\partial}_{\left(J_{\varepsilon}, K_{\varepsilon}\right)}$ as $D_{u} \bar{\partial}$ if there is no danger of confusion.

The method is similar to that of gluing two $\boldsymbol{J}$-holomorphic discs in $M$ using cylindrical domains, as in Section 29 in [FOOO2]. Indeed, we glue each of the two ends of our local model $u_{0}$ with the outer curves $u_{-}$and $u_{+}$, respectively, and the gluing at two ends are somewhat independent, so locally our construction looks like gluing two curves $u_{0}$ with $u_{-}$or $u_{0}$ with $u_{+}$, respectively.

We introduce various cut-off functions to patch the approximate right inverse.

$$
\chi_{S}^{+}(\tau)= \begin{cases}0, & \tau \leq S-1 \\ 1, & \tau \geq S+1\end{cases}
$$

with $\left|\nabla \chi_{S}^{+}\right| \leq 1$, and put

$$
\chi_{S}^{-}(\tau)=1-\chi_{S}^{+}(\tau), \quad \chi_{S}^{0}(\tau)=1-\chi_{S}^{+}(\tau)-\chi_{-S}^{-}(\tau) .
$$

In the following of this section, it is important to let $S$ have the same order as $R(\varepsilon)$ ) but smaller than $R(\varepsilon)$. For convenience we set

$$
S=\frac{1}{4} R(\varepsilon) .
$$

We also need some "transporting" and "combining" operators to define the approximate right inverse. Recall that when $\tau \in\left[\frac{1}{2} R(\varepsilon), \frac{3}{2} R(\varepsilon)\right]$, the shifted outer curve $u_{+}^{\varepsilon}(\tau, t)=u_{+}(\tau-2 R(\varepsilon), t)$, the scaled local model $u_{0}^{\varepsilon}(\tau, t)=\varepsilon u_{0}(\tau, t)$ and the approximate solution $u_{\text {app }}^{\varepsilon}(\tau, t)$ are exponentially close to each other in the cylindrical metric $g_{\varepsilon_{0}, \varepsilon, p}$. Therefore, for any $|\tau|<\frac{1}{2} R(\varepsilon)$, we can define the transform

$$
J_{0, \varepsilon}^{S}: \Gamma\left(\Lambda^{0,1}\left(\left(u_{0}\right)^{*} T \mathbb{C}^{n}\right)\right) \rightarrow \Gamma\left(\Lambda^{0,1}\left(\left(u_{\mathrm{app}}^{\varepsilon}\right)^{*} T M\right)\right)
$$

in the following way: given any $\eta$ in $\Gamma\left(\Lambda^{0,1}\left(\left(u_{0}\right)^{*} T \mathbb{C}^{n}\right)\right)$, we push forward it to $\Gamma\left(\Lambda^{0,1}\left(\left(u_{0}^{\varepsilon}\right)^{*} T \mathbb{C}^{n}\right)\right)$ using the scaling $\varepsilon: \mathbb{C}^{n} \rightarrow \mathbb{C}^{n}, u_{0} \rightarrow u_{0}^{\varepsilon}$. Then we cut it by $\chi_{R(\varepsilon)+S}^{0}$ and use parallel transport $\mathrm{Pal}_{0, \varepsilon}$ along minimal geodesics connecting $u_{0}^{\varepsilon}(\tau, t)$ and $u_{\text {app }}^{\varepsilon}(\tau, t)$ to get a section on $\Gamma\left(\Lambda^{0,1}\left(\left(u_{\text {app }}^{\varepsilon}\right)^{*} T M\right)\right)$. In short, we denote

$$
\left.J_{0, \varepsilon}^{S} \eta=\operatorname{Pal}_{0, \varepsilon}\left(\chi_{R(\varepsilon)+S}^{0}\left((\varepsilon)_{*} \eta\right)\right)\right) .
$$


Similarly, we can define the transform

$$
J_{+, \varepsilon}^{S}: \Gamma\left(\Lambda^{0,1}\left(\left(u_{+}\right)^{*} T M\right)\right) \rightarrow \Gamma\left(\Lambda^{0,1}\left(\left(u_{\mathrm{app}}^{\varepsilon}\right)^{*} T M\right)\right)
$$

as the following: for any $\eta(\tau, t) \in \Gamma\left(\Lambda^{0,1}\left(\left(u_{+}\right)^{*} T M\right)\right)$, we shift it to $\eta(\tau-$ $2 R(\varepsilon)$ ) and regard it as a one-form on $\left.u_{+}^{\varepsilon}(\tau, t)=u_{+}(\tau-2 R(\varepsilon), t)\right)$. We cut it by $\chi_{R(\varepsilon)-S}^{+}$and use parallel transport $\mathrm{Pal}_{+, \varepsilon}$ along minimal geodesics connecting $u_{+}^{\varepsilon}(\tau, t)$ and $u_{\text {app }}^{\varepsilon}(\tau, t)$ to get a section on $\Gamma\left(\Lambda^{0,1}\left(\left(u_{\text {app }}^{\varepsilon}\right)^{*} T M\right)\right)$. In short,

$$
J_{+, \varepsilon}^{S} \eta=\operatorname{Pal}_{+, \varepsilon}\left(\chi_{R(\varepsilon)-S}^{+}(\eta(\tau-2 R(\varepsilon), t))\right) .
$$

Similarly, we define

$$
J_{-, \varepsilon}^{S}: \Gamma\left(\Lambda^{0,1}\left(\left(u_{-}^{\varepsilon}\right)^{*} T \mathbb{C}^{n}\right)\right) \rightarrow \Gamma\left(\Lambda^{0,1}\left(\left(u_{\mathrm{app}}^{\varepsilon}\right)^{*} T M\right)\right)
$$

to be

$$
J_{-, \varepsilon}^{S} \eta=\operatorname{Pal}_{-, \varepsilon}\left(\chi_{-R(\varepsilon)+S}^{-}(\eta(\tau+2 R(\varepsilon), t))\right) .
$$

For the reversed ones

$$
\begin{aligned}
& J_{\varepsilon, 0}^{S}: \Gamma\left(\Lambda^{0,1}\left(\left(u_{\text {app }}^{\varepsilon}\right)^{*} T M\right)\right) \rightarrow \Gamma\left(\Lambda^{0,1}\left(\left(u_{0}\right)^{*} \mathbb{C}^{n}\right)\right), \\
& J_{\varepsilon,+}^{S}: \Gamma\left(\Lambda^{0,1}\left(\left(u_{\text {app }}^{\varepsilon}\right)^{*} T M\right)\right) \rightarrow \Gamma\left(\Lambda^{0,1}\left(\left(u_{+}\right)^{*} T M\right)\right), \\
& J_{\varepsilon,-}^{S}: \Gamma\left(\Lambda^{0,1}\left(\left(u_{\text {app }}^{\varepsilon}\right)^{*} T M\right)\right) \rightarrow \Gamma\left(\Lambda^{0,1}\left(\left(u_{-}\right)^{*} T M\right)\right),
\end{aligned}
$$

the definitions are similar. For example for $J_{\varepsilon,+}^{S}$, for any $\eta$ in $\Gamma\left(\Lambda^{0,1}\left(\left(u_{\mathrm{app}}^{\varepsilon}\right)^{*}\right.\right.$ $T M)$ ), we cut it by $\chi_{R(\varepsilon)-S}^{+}$then use parallel transport $\mathrm{Pal}_{\varepsilon,+}$ from $u_{\text {app }}^{\varepsilon}$ to $u_{+}^{\varepsilon}$ to get an element in $\Gamma\left(\Lambda^{0,1}\left(\left(u_{+}^{\varepsilon}\right)^{*} T M\right)\right)$, and then shift it to $\Gamma\left(\Lambda^{0,1}\left(\left(u_{+}\right)^{*} T M\right)\right)$. In short,

$$
J_{\varepsilon,+}^{S} \eta=\operatorname{Pal}_{\varepsilon,+}\left(\chi_{R(\varepsilon)-S}^{+}(\tau+2 R(\varepsilon))(\eta(\tau+2 R(\varepsilon), t))\right) .
$$

It is easy to check the following identities:

$$
\begin{aligned}
J_{0, \varepsilon}^{S} \circ J_{\varepsilon, 0}^{S}\left(\chi_{R(\varepsilon)}^{0} \eta\right) & =\chi_{R(\varepsilon)}^{0} \eta, \\
J_{+, \varepsilon}^{S} \circ J_{\varepsilon,+}^{S}\left(\chi_{R(\varepsilon)}^{+} \eta\right) & =\chi_{R(\varepsilon)}^{+} \eta, \\
J_{-, \varepsilon}^{S} \circ J_{\varepsilon,-}^{S}\left(\chi_{R(\varepsilon)}^{-} \eta\right) & =\chi_{R(\varepsilon)}^{-} \eta .
\end{aligned}
$$

For an enhanced nodal Floer trajectory $u=\left(u_{-}, u_{0}, u_{+}\right)$, the "combining" operator

$$
I^{S}: T_{u} W_{\alpha}^{1, p} \rightarrow T_{u_{\mathrm{app}}^{\varepsilon}} W_{\alpha_{\delta, \varepsilon}}^{1, p}
$$

is defined as the following: for $\xi=\left(\xi_{-}, \xi_{0}, \xi_{+}\right) \in T_{u} \mathcal{B}_{\text {noal }}$ (defined in (8.17)), i.e.,

$(10.44) \xi_{-} \in T_{u_{-}} W_{\alpha}^{1, p}\left(\dot{\Sigma}, \widetilde{M} ; z_{-}\right), \xi_{0} \in T_{u_{0}} \mathcal{B}_{\mathrm{lmd}}$, and $\xi_{+} \in W_{\alpha}^{1, p}\left(\dot{\Sigma}, \widetilde{M} ; z_{+}\right)$ 
with the matching condition

$$
\xi_{-}(+\infty, t)=\xi_{0}(-\infty, t)=V^{-}(t), \quad \xi_{+}(-\infty, t)=\xi_{0}(+\infty, t)=V^{+}(t),
$$

then by the above $\varepsilon$-scaling and $\pm 2 R(\varepsilon)$ shifting we can regard $\xi_{-}, \xi_{0}$ and $\xi_{+}$as the elements in $T_{u_{-}^{\varepsilon}} W_{\alpha_{\varepsilon}}^{1, p}\left(\dot{\Sigma}, \widetilde{M} ; z_{-}\right), T_{u_{0}^{\varepsilon}} \mathcal{B}_{\text {lmd }}$ and $T_{u_{+}^{\varepsilon}} W_{\alpha_{\varepsilon}}^{1, p}\left(\dot{\Sigma}, \widetilde{M} ; z_{+}\right)$, respectively, with the same matching condition (10.45) (here the weighting function $\alpha_{\varepsilon}(\tau)$ is the shifting of the weighting function $\alpha$, namely $\alpha_{\varepsilon}(\tau)=$ $\alpha(\tau- \pm 2 R(\varepsilon))$ for $u_{ \pm}^{\varepsilon}$, respectively). For convenience we still denote them by $\xi_{-}, \xi_{0}$ and $\xi_{+}$. Then we define

$$
\begin{aligned}
& I^{S}\left(\xi_{-}, V^{-}, \xi_{0}, V^{+}, \xi_{+}\right)(\tau, t) \\
& \quad= \begin{cases}\xi_{+}(\tau, t), & \tau \in\left[\frac{5}{4} R(\varepsilon),+\infty\right), \\
V^{+}+\chi_{R(\varepsilon)+S}^{-}\left(\mathrm{Pal}_{0, \varepsilon}\left(\xi_{0}\right)-V^{+}\right) & \\
\quad+\chi_{R(\varepsilon)-S}^{+}\left(\mathrm{Pal}_{+, \varepsilon}\left(\xi_{+}\right)-V^{+}\right), & \tau \in\left[\frac{3}{4} R(\varepsilon), \frac{5}{4} R(\varepsilon)\right], \\
\xi_{0}(\tau, t), & \tau \in\left[-\frac{3}{4} R(\varepsilon), \frac{3}{4} R(\varepsilon)\right], \\
V^{-}+\chi_{-R(\varepsilon)-S}^{+}\left(\mathrm{Pal}_{0, \varepsilon}\left(\xi_{0}\right)-V^{-}\right) & \\
\quad+\chi_{-R(\varepsilon)+S}^{-}\left(\mathrm{Pal}_{-, \varepsilon}\left(\xi_{-}\right)-V^{-}\right), & \tau \in\left[-\frac{3}{4} R(\varepsilon),-\frac{1}{4} R(\varepsilon)\right], \\
\xi_{-}(\tau, t), & \tau \in\left(-\infty,-\frac{5}{4} R(\varepsilon)\right] .\end{cases}
\end{aligned}
$$

In the above expression the $V^{+}$should be regarded as a vector field obtained by the parallel transport of $V^{+}(t)$ to $u_{\text {app }}^{\varepsilon}(\tau, t)$ in the following way: In the cylindrical coordinate $(s, \Theta)$ of $M \backslash\{p\}$, write $V^{+}(t)=\left(V_{\mathbb{R}}^{+}, V_{\mathcal{R}_{1}(\lambda)}^{+}(t)\right) \in$ $T_{\left(\tau_{+}, \gamma_{+}(t)\right)}\left(\mathbb{R} \times S^{2 n-1}\right)$. For $u_{\text {app }}^{\varepsilon}$ close enough to $\left(u_{-}, u_{0}, u_{+}\right)$, and $|\tau| \in$ $\left[\frac{3}{4} R(\varepsilon), \frac{5}{4} R(\varepsilon)\right], u_{\mathrm{app}}^{\varepsilon}(\tau, t)$ is in the the cylindrical metric part of $\left(M, g_{\varepsilon_{0}, \varepsilon, p}\right)$, which can be isometrically identified as a part of $\mathbb{R} \times S^{2 n-1}$. Then we use the connection of $\mathbb{R} \times S^{2 n-1}$ to do the parallel transport, namely, transport $V_{\mathbb{R}}^{+}$trivially, and transport $V_{\mathcal{R}_{1}(\lambda)}^{+}(t)$ along the minimal geodesic connecting $\gamma_{+}(t)$ and $\Theta\left(u_{\text {app }}^{\varepsilon}(\tau, t)\right)$ in $S^{2 n-1}$. We remark that the above intropolation $I^{S}$ also performs on the common base variation $v \in T_{p} M$ shared by $\xi_{-}, \xi_{0}$ and $\xi_{+}$, resulting in $v$ again.

Remark 10.6. The somewhat complicated interpolation among $V^{ \pm}, \xi_{0}$ and $\xi_{ \pm}$using $\chi_{ \pm R(\varepsilon) \pm S}^{ \pm}$instead of the simple interpolation between $\xi_{0}$ and $\xi_{ \pm}$ using $\chi_{ \pm R(\varepsilon)}^{ \pm}$is responsible for the better accuracy of our approximate right inverse with respect to the exponential weight $\alpha_{\delta, \varepsilon}$, because it makes the interpolation happen at the places $\tau= \pm R(\varepsilon) \pm S$ avoiding the "peaks" of the weight function $\alpha_{\delta, \varepsilon}$ at $\tau= \pm R(\varepsilon)$. We will see the advantage of this while doing the estimates of the approximate right inverse later.

Now we define the approximate right inverse $Q^{\varepsilon}$ for $\varepsilon>0$ using the right inverse $\left.Q^{\varepsilon}\right|_{\varepsilon=0}$ on $\left(u_{-}, u_{0}, u_{+}\right)$defined (8.19). For $\eta \in L_{\alpha_{\delta, \varepsilon}}^{p}\left(\Lambda^{0,1}\left(u_{\mathrm{app}}^{\varepsilon}\right)^{*} T M\right)$, 
we let

$$
\begin{aligned}
Q^{\varepsilon}(\eta) & =I^{S}\left(\left.Q^{\varepsilon}\right|_{\varepsilon=0}\left(J_{\varepsilon,-}^{S}\left(\chi_{R(\varepsilon)}^{-} \eta\right), J_{\varepsilon, 0}^{S}\left(\chi_{R(\varepsilon)}^{0} \eta\right), J_{\varepsilon,+}^{S}\left(\chi_{R(\varepsilon)}^{+} \eta\right)\right)\right) \\
& =I^{S}\left(Q_{-} \circ J_{\varepsilon,-}^{S}\left(\chi_{R(\varepsilon)}^{-} \eta\right), V_{\varepsilon}^{-}, Q_{0} \circ J_{\varepsilon, 0}^{S}\left(\chi_{R(\varepsilon)}^{0} \eta\right), V_{\varepsilon}^{+}, Q_{+} \circ J_{\varepsilon,+}^{S}\left(\chi_{R(\varepsilon)}^{+} \eta\right)\right) \\
& =I^{S}\left(\xi_{\varepsilon,-}, V_{\varepsilon}^{-}, \xi_{\varepsilon, 0}, V_{\varepsilon}^{+}, \xi_{\varepsilon,+}, v\right),
\end{aligned}
$$

where

$\xi_{\varepsilon,-}=Q_{-} \circ J_{\varepsilon,-}^{S}\left(\chi_{R(\varepsilon)}^{-} \eta\right), \xi_{\varepsilon, 0}=Q_{0} \circ J_{\varepsilon, 0}^{S}\left(\chi_{R(\varepsilon)}^{0} \eta\right), \xi_{\varepsilon,+}=Q_{+} \circ J_{\varepsilon,+}^{S}\left(\chi_{R(\varepsilon)}^{+} \eta\right)$ and $V_{\varepsilon}^{+}, V_{\varepsilon}^{-}$are their matching asymptotes at infinity, namely

$$
V_{\varepsilon}^{-}(t)=\xi_{\varepsilon,-}(+\infty, t)=\xi_{\varepsilon, 0}(-\infty, t), \quad V_{\varepsilon}^{+}(t)=\xi_{\varepsilon,+}(-\infty, t)=\xi_{\varepsilon, 0}(+\infty, t),
$$

and $v \in T_{p} M$ is the common base variation shared by $\xi_{-}, \xi_{0}$ and $\xi_{+}$.

Convention of uniform constants: In the remaining part of this subsection, in many estimates there are different constants $C$ 's. The exact values are not important. The importance is that all of them are independent on $\varepsilon$. For this reason, we just denote them by the same symbol $C$ and shouldn't cause problems.

We show the norm of $Q^{\varepsilon}$ is uniformly bounded:

Proposition 10.1. There exists a constant $C=C(\delta)>0$ independent on E such that

$$
\left\|Q^{\varepsilon}(\eta)\right\|_{1, p, \alpha_{\delta, \varepsilon}} \leq C\|\eta\|_{p, \alpha_{\delta, \varepsilon}},
$$

for all $\eta \in L_{\alpha_{\delta, \varepsilon}}^{p}\left(\Lambda^{0,1}\left(u_{\mathrm{app}}^{\varepsilon}\right)^{*} T M\right)$.

Proof. We first show that for $\left(\xi_{-}, \xi_{0}, \xi_{+}\right)$in (10.44) with matching condition (10.45), and $S=\frac{1}{4} R(\varepsilon)$,

$$
\left\|I^{S}\left(\xi_{-}, V^{-}, \xi_{0}, V^{+}, \xi_{+}\right)\right\|_{1, p, \alpha_{\delta, \varepsilon}} \leq C\left(\left\|\xi_{-}\right\|_{1, p, \alpha}+\left\|\xi_{0}\right\|_{1, p, \delta}+\left\|\xi_{+}\right\|_{1, p, \alpha}\right),
$$

where $C$ is independent on $\varepsilon$.

By the definition of $I^{S}$, to prove (10.48), it is enough to estimate the norm of the right-hand side on $[-2 R(\varepsilon), 2 R(\varepsilon)] \times S^{1}$. Let $\xi_{\text {app }}^{\varepsilon}=$ $\left.I^{S}\left(\xi_{-}, V^{-}, \xi_{0}, V^{+}, \xi_{+}\right)\right)$. Then

$\xi_{\text {app }}^{\varepsilon}(\tau, t)=\operatorname{Pal}_{0, \varepsilon}\left(\xi_{0}\right)+\mathrm{Pal}_{+, \varepsilon}\left(\xi_{+}\right)-V^{+}, \quad$ for $\tau \in[R(\varepsilon)-1, R(\varepsilon)+1]$, $\xi_{\text {app }}^{\varepsilon}(\tau, t)=\operatorname{Pal}_{0, \varepsilon}\left(\xi_{0}\right)+\mathrm{Pal}_{-, \varepsilon}\left(\xi_{-}\right)-V^{-}, \quad$ for $\tau \in[-R(\varepsilon)-1,-R(\varepsilon)+1]$.

By Sobolev inequality and the definitions of the norms $\|\cdot\|_{1, p, \delta}$ and $\|\cdot\|_{1, p, \alpha}$, we have the point estimate

$$
\left|\xi_{\text {app }}^{\varepsilon}( \pm R(\varepsilon))-V^{ \pm}\right| \leq C \mathrm{e}^{\frac{-2 \pi \delta R(\varepsilon)}{p}}\left(\left\|\xi_{0}\right\|_{1, p, \delta}+\left\|\xi_{ \pm}\right\|_{1, p, \alpha}\right) .
$$


We also have the energy estimate

$$
\begin{aligned}
& {\left[\int _ { [ 0 , 2 R ( \varepsilon ) ] \times S ^ { 1 } } \alpha _ { \delta , \varepsilon } \cdot \left(\left|\nabla\left(\mathrm{Pal}_{u_{\mathrm{app}}^{\varepsilon}(\tau, t)}\left(\xi_{\mathrm{app}}^{\varepsilon}(R(\varepsilon))\right)-V^{+}\right)\right|^{p}\right.\right.} \\
& \left.\left.\quad+\left|\mathrm{Pal}_{u_{\mathrm{app}}^{\varepsilon}(\tau, t)}\left(\xi_{\mathrm{app}}^{\varepsilon}(R(\varepsilon))\right)-V^{+}\right|^{p}\right) d \tau d t\right]^{\frac{1}{p}} \\
& \quad \leq C\left(\left\|\xi_{-}\right\|_{1, p, \alpha}+\left\|\xi_{0}\right\|_{1, p, \delta}+\left\|\xi_{+}\right\|_{1, p, \alpha}\right)
\end{aligned}
$$

and a similar inequality for $V^{-}$in $[-2 R(\varepsilon), 0]$.

Postponing the proof of (10.49) to the next lemma, it is enough to estimate

$$
\int_{[0,2 R(\varepsilon)] \times S^{1}} \alpha_{\delta, \varepsilon} \cdot\left(\left|\nabla\left(\xi_{\mathrm{app}}^{\varepsilon}-V^{+}\right)\right|^{p}+\left|\xi_{\mathrm{app}}^{\varepsilon}-V^{+}\right|^{p}\right) d \tau d t
$$

and

$$
\int_{[-2 R(\varepsilon), 0] \times S^{1}} \alpha_{\delta, \varepsilon} \cdot\left(\left|\nabla\left(\xi_{\text {app }}^{\varepsilon}-V^{-}\right)\right|^{p}+\left|\xi_{\text {app }}^{\varepsilon}-V^{-}\right|^{p}\right) d \tau d t .
$$

Since the above two terms are similar, we only estimate the first term. The first term is estimated by

$$
\begin{aligned}
& C \int_{[0,2 R(\varepsilon)] \times S^{1}} e^{2 \pi \delta|\tau|} \cdot\left(\left|\chi_{R(\varepsilon)+S}^{-}(\tau)\left(\xi_{0}-V^{+}\right)\right|^{p}\right. \\
& \left.\quad+\left|\nabla\left(\chi_{R(\varepsilon)+S}^{-}(\tau)\left(\xi_{0}-V^{+}\right)\right)\right|^{p}\right) d \tau d t \\
& \quad+C \int_{[0,2 R(\varepsilon)] \times S^{1}} \alpha(\tau) \cdot\left(\left|\chi_{R(\varepsilon)-S}^{+}(\tau)\left(\xi_{+}-V^{+}\right)\right|^{p}\right. \\
& \left.\quad+\left|\nabla\left(\chi_{R(\varepsilon)-S}^{+}(\tau)\left(\xi_{+}-V^{+}\right)\right)\right|^{p}\right) d \tau d t .
\end{aligned}
$$

This is because the weight $\alpha_{\delta, \varepsilon}(\tau, t)$ is estimated by $\mathrm{e}^{2 \pi \delta|\tau|}$ and $\alpha(\tau)$, respectively, on the support of $\chi_{R(\varepsilon)+S}^{-}(\tau)$ and $\chi_{R(\varepsilon)-S}^{+}(\tau)$. Then it is easy to estimate the above expression by the right-hand side of (10.48).

To compete the proof of Proposition 10.1 it is enough to show

$$
\left\|J_{\varepsilon, \pm}^{S}\left(\chi_{R(\varepsilon)}^{ \pm} \eta\right)\right\|_{p, \alpha} \leq B\|\eta\|_{p, \alpha_{\delta, \varepsilon}}, \quad\left\|J_{\varepsilon, 0}^{S}\left(\chi_{R(\varepsilon)}^{0} \eta\right)\right\|_{p, \delta} \leq B\|\eta\|_{p, \alpha_{\delta, \varepsilon}} .
$$

for some fixed constant $B$. Note that

$$
\begin{gathered}
J_{\varepsilon, \pm}^{S}\left(\chi_{R(\varepsilon)}^{ \pm} \eta\right)=\operatorname{Pal}_{\varepsilon, \pm}\left(\chi_{R(\varepsilon)}^{ \pm} \eta\right), \\
J_{\varepsilon, 0}^{S}\left(\chi_{R(\varepsilon)}^{0} \eta\right)=\operatorname{Pal}_{\varepsilon, 0}\left(\chi_{R(\varepsilon)}^{0} \eta\right),
\end{gathered}
$$

and the weight $\alpha_{\delta, \varepsilon}(\tau)$ restricting on the support of $\chi_{R(\varepsilon)}^{-}, \chi_{R(\varepsilon)}^{0}, \chi_{R(\varepsilon)}^{+}$agrees with the weights of $u_{-}^{\varepsilon}, u_{0}^{\varepsilon}$ and $u_{+}^{\varepsilon}$, respectively (more precisely, they agree in the sense that their ratio remains in the finite interval $\left.\left[\frac{1}{2}, \frac{3}{2}\right]\right),(10.50)$ follows by taking $B=2$. 
Lemma 10.3. There exists a constant $C$ independent on $\varepsilon$ (but may be dependent on $\delta$ ) such that

$$
\begin{aligned}
& {\left[\int _ { [ 0 , 2 R ( \varepsilon ) ] \times S ^ { 1 } } \alpha _ { \delta , \varepsilon } \cdot \left(\left|\nabla\left(\mathrm{Pal}_{u_{\mathrm{app}}^{\varepsilon}(\tau, t)}\left(\xi_{\mathrm{app}}^{\varepsilon}(R(\varepsilon))\right)-V^{+}\right)\right|^{p}\right.\right.} \\
& \left.\left.\quad+\left|\mathrm{Pal}_{u_{\mathrm{app}}^{\varepsilon}(\tau, t)}\left(\xi_{\mathrm{app}}^{\varepsilon}(R(\varepsilon))\right)-V^{+}\right|^{p}\right) d \tau d t\right]^{\frac{1}{p}} \\
& \leq C\left(\left\|\xi_{-}\right\|_{1, p, \alpha}+\left\|\xi_{0}\right\|_{1, p, \delta}+\left\|\xi_{+}\right\|_{1, p, \alpha}\right)
\end{aligned}
$$

and

$$
\begin{aligned}
& {\left[\int _ { [ - 2 R ( \varepsilon ) , 0 ] \times S ^ { 1 } } \alpha _ { \delta , \varepsilon } \cdot \left(\left|\nabla\left(\mathrm{Pal}_{u_{\mathrm{app}}^{\varepsilon}(\tau, t)}\left(\xi_{\mathrm{app}}^{\varepsilon}(-R(\varepsilon))\right)-V^{-}\right)\right|^{p}\right.\right.} \\
& \left.\left.\quad+\left|\mathrm{Pal}_{u_{\mathrm{app}}^{\varepsilon}(\tau, t)}\left(\xi_{\mathrm{app}}^{\varepsilon}(-R(\varepsilon))\right)-V^{-}\right|^{p}\right) d \tau d t\right]^{\frac{1}{p}} \\
& \quad \leq C\left(\left\|\xi_{-}\right\|_{1, p, \alpha}+\left\|\xi_{0}\right\|_{1, p, \delta}+\left\|\xi_{+}\right\|_{1, p, \alpha}\right) .
\end{aligned}
$$

Proof. We only prove the first inequality; the second one is similar. Put

$$
V(\tau, t)=\operatorname{Pal}_{u_{\mathrm{app}}^{\varepsilon}(\tau, t)}\left(\xi_{\mathrm{app}}^{\varepsilon}(R(\varepsilon))\right)-\mathrm{Pal}_{u_{\mathrm{app}}^{\varepsilon}(\tau, t)} V^{+} .
$$

Then for $\tau \in[R(\varepsilon)-S, R(\varepsilon)+S]$,

$$
\begin{aligned}
|V(\tau, t)|=\mid & \chi_{R(\varepsilon)+S}^{-}(\tau)\left(\mathrm{Pal}_{u_{\mathrm{app}}^{\varepsilon}(\tau, t)}\left(\xi_{0}(R(\varepsilon))\right)-\mathrm{Pal}_{u_{\mathrm{app}}^{\varepsilon}(\tau, t)} V^{+}\right) \\
& +\chi_{R(\varepsilon)-S}^{+}(\tau)\left(\mathrm{Pal}_{u_{\mathrm{app}}^{\varepsilon}(\tau, t)}\left(\xi_{+}(-R(\varepsilon))\right)-\mathrm{Pal}_{u_{\mathrm{app}}^{\varepsilon}(\tau, t)} V^{+}\right) \mid \\
\leq \mid & \mathrm{Pal}_{u_{\mathrm{app}}^{\varepsilon}(\tau, t)}\left(\xi_{0}(R(\varepsilon))\right)-\mathrm{Pal}_{u_{\mathrm{app}}^{\varepsilon}(\tau, t)} V^{+} \mid \\
& +\left|\mathrm{Pal}_{u_{\mathrm{app}}^{\varepsilon}(\tau, t)}\left(\xi_{+}(-R(\varepsilon))\right)-\mathrm{Pal}_{u_{\mathrm{app}}^{\varepsilon}(\tau, t)} V^{+}\right| .
\end{aligned}
$$

Equation (10.53) still holds outside $[R(\varepsilon)-S, R(\varepsilon)+S]$ by the definition of $V(\tau, t)$.

For the third row in (10.53), using the invariance of vector norm under parallel transport from the tangent space at $u_{\text {app }}^{\varepsilon}(\tau, t)$ in $M$ to the tangent space at $u_{0}^{\varepsilon}(R(\varepsilon))$ in $M$, we get

$$
\begin{aligned}
& \left|\mathrm{Pal}_{u_{\mathrm{app}}^{\varepsilon}(\tau, t)}\left(\xi_{0}(R(\varepsilon))\right)-\mathrm{Pal}_{u_{\mathrm{app}}^{\varepsilon}(\tau, t)} V^{+}\right| \\
& \quad=\left|\xi_{0}(R(\varepsilon))-\mathrm{Pal}_{u_{0}^{\varepsilon}(R(\varepsilon))} \mathrm{Pal}_{u_{\mathrm{app}}^{\varepsilon}(\tau, t)} V^{+}\right| \\
& \quad \leq\left|\xi_{0}(R(\varepsilon))-V^{+}\right|+\left|V^{+}-\mathrm{Pal}_{u_{0}^{\varepsilon}(R(\varepsilon))} \mathrm{Pal}_{u_{\mathrm{app}}^{\varepsilon}(\tau, t)} V^{+}\right| .
\end{aligned}
$$

To estimate the second term of the last inequality, we only need to consider the parallel transport of $V^{+}$in the $S^{2 n-1}$ component, since the $\mathbb{R}$ component has trivial connection. We need to compare the difference of the parallel transport of $V_{\mathcal{R}_{\lambda}}^{+}$along two different geodesic paths in $S^{2 n-1}$ : one is from 
$\gamma_{+}(t)$ to $\Theta \circ u_{0}^{\varepsilon}(R(\varepsilon), t)$, the other is from $\gamma_{+}(t)$ to $\Theta \circ u_{\text {app }}^{\varepsilon}(\tau, t)$ and then to $\Theta \circ u_{0}^{\varepsilon}(R(\varepsilon), t)$. Since parallel transport is governed by a first-order linear ODE, from the exponential convergence of $\Theta \circ u_{\mathrm{app}}^{\varepsilon}(\tau, t)$ and $\Theta \circ u_{0}^{\varepsilon}(R(\tau, t)$ to $\gamma_{+}(t)$, and the $C^{0}$ continuous dependence of solutions of ODE on its coefficients, we get

$$
\begin{aligned}
& \left|\mathrm{Pal}_{u_{\mathrm{app}}^{\varepsilon}(\tau, t)}\left(\xi_{0}(R(\varepsilon))\right)-\mathrm{Pal}_{u_{\mathrm{app}}^{\varepsilon}(\tau, t)} V^{+}\right| \\
& \quad \leq\left|\xi_{0}(R(\varepsilon))-V^{+}\right|+C \mathrm{e}^{\frac{-2 \pi c(R(\varepsilon)-|\tau-R(\varepsilon)|)}{p}}\left|V^{+}\right| .
\end{aligned}
$$

Similar argument yields

$$
\begin{aligned}
& \left|\mathrm{Pal}_{u_{\mathrm{app}}^{\varepsilon}(\tau, t)}\left(\xi_{+}(-R(\varepsilon))\right)-\mathrm{Pal}_{u_{\mathrm{app}}^{\varepsilon}(\tau, t)} V^{+}\right| \\
& \quad \leq C\left|\xi_{+}(-R(\varepsilon))-V^{+}\right|+C \mathrm{e}^{\frac{-2 \pi c(R(\varepsilon)-|\tau-R(\varepsilon)|)}{p}}\left|V^{+}\right| .
\end{aligned}
$$

It is obvious that (10.55) and (10.56) hold when $\tau$ is outside $[R(\varepsilon)-S, R(\varepsilon)+$ $S]$, by definitions of the cut functions. Plugging these in (10.53) we have the point estimate

$$
\begin{aligned}
|V(\tau, t)| \leq & C\left(\left|\xi_{0}(R(\varepsilon))-V^{+}\right|+\left|\xi_{+}(-R(\varepsilon))-V^{+}\right|\right) \\
& +C \mathrm{e}^{\frac{-2 \pi c(R(\varepsilon)-|\tau-R(\varepsilon)|)}{p}}\left|V^{+}\right|
\end{aligned}
$$

for all $\tau$. Similarly, we can estimate $|\nabla V(\tau, t)|$, using the $C^{1}$ continuous dependence of solutions of ODE on its coefficients, and the $C^{1}$ exponential convergence of $\Theta \circ u_{\text {app }}^{\varepsilon}(\tau, t)$ and $\Theta \circ u_{0}^{\varepsilon}\left(R(\tau, t)\right.$ to $\gamma_{+}(t)$. We get

$$
\begin{aligned}
|\nabla V(\tau, t)| \leq & C\left(\left|\xi_{0}(R(\varepsilon))-V^{+}\right|+\left|\left(\nabla \xi_{0}\right)(R(\varepsilon))-\nabla V^{+}\right|\right) \\
& +C\left(\left|\xi_{+}(-R(\varepsilon))-V^{+}\right|+\left|\left(\nabla \xi_{+}\right)(-R(\varepsilon))-\nabla V^{+}\right|\right) \\
& +C \mathrm{e}^{\frac{-2 \pi c(R(\varepsilon)-|\tau-R(\varepsilon)|)}{p}}\left|V^{+}\right| .
\end{aligned}
$$

Now we integrate $(10.57)$ and $(10.58)$ on $[0,2 R(\varepsilon)] \times S^{1}$ to get the $W_{\alpha_{\delta, \varepsilon}}^{1, p}$ estimate of $V(\tau, t)$. We have

$$
\begin{aligned}
& \int_{[0,2 R(\varepsilon)] \times S^{1}}\left(|V(\tau, t)|^{p}+|\nabla V(\tau, t)|^{p}\right) \alpha_{\delta, \varepsilon}(\tau) d \tau d t \\
& \leq C\left[\left|\xi_{0}(R(\varepsilon))-V^{+}\right|^{p}+\left|\xi_{+}(-R(\varepsilon))-V^{+}\right|^{p}\right. \\
& \left.\quad+\left|\left(\nabla \xi_{0}\right)(R(\varepsilon))-\nabla V^{+}\right|^{p}+\left|\left(\nabla \xi_{+}\right)(-R(\varepsilon))-\nabla V^{+}\right|^{p}\right] \frac{\mathrm{e}^{2 \pi \delta R(\varepsilon)}}{\delta} \\
& \quad+\frac{C}{c-\delta} \mathrm{e}^{-2 \pi(c-\delta) R(\varepsilon)}\left|V^{+}\right|^{p}
\end{aligned}
$$


which becomes

$$
\begin{aligned}
& C\left[\left(\left|\xi_{0}(R(\varepsilon))-V^{+}\right|^{p}+\left|\left(\nabla \xi_{0}\right)(R(\varepsilon))-\nabla V^{+}\right|^{p}\right)\right. \\
&\left.\quad+\left(\left|\xi_{+}(-R(\varepsilon))-V^{+}\right|^{p}+\left|\left(\nabla \xi_{+}\right)(-R(\varepsilon))-\nabla V^{+}\right|^{p}\right)\right] \frac{\mathrm{e}^{2 \pi \delta R(\varepsilon)}}{\delta} \\
& \quad+\frac{C}{c-\delta} \mathrm{e}^{-2 \pi(c-\delta) R(\varepsilon)\left|V^{+}\right|^{p}} \\
& \leq C\left[\int_{[R(\varepsilon)-1, R(\varepsilon)+1] \times S^{1}}\left(\left|\xi_{0}(\tau, t)-V^{+}\right|^{p}+\left|\left(\nabla \xi_{0}\right)(\tau, t)-\nabla V^{+}\right|^{p}\right)\right. \\
& \quad \times \mathrm{e}^{-2 \pi \delta(R(\varepsilon)-\tau)} d \tau d t+\int_{[-R(\varepsilon)-1,-R(\varepsilon)+1] \times S^{1}}\left(\left|\xi_{+}(\tau, t)-V^{+}\right|^{p}\right. \\
&\left.\left.\quad+\left|\left(\nabla \xi_{+}\right)(\tau, t)-\nabla V^{+}\right|^{p}\right) \mathrm{e}^{-2 \pi \delta(R(\varepsilon)+\tau)} d \tau d t\right] \\
& \quad \times \frac{\mathrm{e}^{2 \pi \delta R(\varepsilon)}}{\delta}+\frac{C}{c-\delta} \mathrm{e}^{-2 \pi(c-\delta) R(\varepsilon)\left|V^{+}\right|^{p}} \\
& \leq C\left[\int_{\mathbb{R} \times S^{1}}\left(\left|\xi_{0}(\tau, t)-V^{+}\right|^{p}+\left|\left(\nabla \xi_{0}\right)(\tau, t)-\nabla V^{+}\right|^{p}\right) \mathrm{e}^{-2 \pi \delta|\tau|} d \tau d t\right. \\
&\left.+\int_{\mathbb{R} \times S^{1}}\left(\left|\xi_{+}(\tau, t)-V^{+}\right|^{p}+\left|\left(\nabla \xi_{+}\right)(\tau, t)-\nabla V^{+}\right|^{p}\right) \alpha(\tau) d \tau d t\right] \\
&+\frac{C}{c-\delta} \mathrm{e}^{-2 \pi(c-\delta) R(\varepsilon)\left|V^{+}\right|^{p},}
\end{aligned}
$$

where in the last inequality we have used Sobolev embedding $W^{1, p} \hookrightarrow C^{0}$, and that $\mathrm{e}^{2 \pi \delta(R(\varepsilon)-\tau)}$ restricted on $[R(\varepsilon)-1, R(\varepsilon)+1]\left(\right.$ or $\mathrm{e}^{2 \pi \delta(R(\varepsilon)+\tau)}$ restricted on $[-R(\varepsilon)-1,-R(\varepsilon)+1])$ is bounded between constants $\mathrm{e}^{-2 \pi \delta}$ and $\mathrm{e}^{2 \pi \delta}$ independent on $\varepsilon$.

Hence

$$
\begin{aligned}
& {\left[\int _ { [ 0 , 2 R ( \varepsilon ) ] \times S ^ { 1 } } \alpha _ { \delta , \varepsilon } \cdot \left(\left|\nabla\left(\mathrm{Pal}_{u_{\mathrm{app}}^{\varepsilon}(\tau, t)}\left(\xi_{\mathrm{app}}^{\varepsilon}(R(\varepsilon))\right)-V^{+}\right)\right|^{p}\right.\right.} \\
& \left.\left.\quad+\left|\mathrm{Pal}_{u_{\mathrm{app}}^{\varepsilon}(\tau, t)}\left(\xi_{\mathrm{app}}^{\varepsilon}(R(\varepsilon))\right)-V^{+}\right|^{p}\right) d \tau d t\right]^{\frac{1}{p}} \\
& \quad \leq C\left(\left\|\xi_{-}\right\|_{1, p, \alpha}+\left\|\xi_{0}\right\|_{1, p, \delta}+\left\|\xi_{+}\right\|_{1, p, \alpha}\right) .
\end{aligned}
$$

The lemma follows.

To show $Q^{\varepsilon}$ is an approximate right inverse, we start with the following lemma concerning the "uniform stabilization" property of the action 
of $Q_{0}$ and $Q_{ \pm}$on compactly supported (or one side compact supported) one-forms $\eta$ :

Lemma 10.4. There exist a constant $C$ independent on $\delta$ and $\varepsilon$ such that

(1) If $\eta_{0} \in L_{\delta}^{p}\left(\Lambda^{0,1}\left(u_{0}\right)^{*} \mathbb{C}^{n}\right)$, then for any $\xi_{0}=Q_{0}\left(\eta_{0}\right)$ with asymptote $V^{ \pm}$ on its two ends, we have

$$
\begin{aligned}
& \left|\xi_{0}(\tau, t)-V^{+}\right| \leq C \mathrm{e}^{\frac{-2 \pi \delta|\tau|}{p}}\left\|\eta_{0}\right\|_{p, \delta}, \quad \text { for } \tau>1, \\
& \left|\xi_{0}(\tau, t)-V^{-}\right| \leq C e^{\frac{-2 \pi \delta|\tau|}{p}}\left\|\eta_{0}\right\|_{p, \delta}, \quad \text { for } \tau<-1,
\end{aligned}
$$

(2) If $\eta_{ \pm} \in L_{\alpha}^{p}\left(\Lambda^{0,1}\left(u_{ \pm}\right)^{*} T M\right)$, then for any $\xi_{ \pm}=Q_{ \pm}\left(\eta_{ \pm}\right)$with the asymptote $V^{ \pm}$as $\tau \rightarrow \pm \infty$, we have

$$
\begin{aligned}
& \left|\xi_{+}(\tau, t)-V^{+}\right| \leq C e^{\frac{-2 \pi \delta|\tau|}{p}}\left\|\eta_{+}\right\|_{p, \alpha}, \quad \text { for } \tau<-1, \\
& \left|\xi_{-}(\tau, t)-V^{-}\right| \leq C e^{\frac{-2 \pi \delta|\tau|}{p}}\left\|\eta_{-}\right\|_{p, \alpha}, \quad \text { for } \tau>1,
\end{aligned}
$$

where $\alpha$ is the weighting function we introduced in Subsection 8.2.

Proof. The proofs of the inequalities are similar, so we just prove (10.61). By definition $\left|\xi_{+}-V^{+}\right| \in W_{\alpha}^{1, p}\left(u_{+}^{*}(T M)\right)$. Suppose $\tau \in(L-1, L+1) \subset(-\infty, 0]$ for some $L$, then $\left|\xi_{+}(\tau, t)-V^{+}\right| \mathrm{e}^{\frac{2 \pi \delta|\tau|}{p}} \in W^{1, p}\left([L-1, L+1] \times S^{1}, u_{+}^{*}(T M)\right)$. By Sobolev embedding $C^{0}\left([L-1, L+1] \times S^{1}, u_{+}^{*}(T M)\right) \hookrightarrow W^{1, p}([L-1, L+$ 1] $\left.\times S^{1}, u_{+}^{*}(T M)\right)$, there is a constant $C$ independent of $L$ and depending only on the metric $g$ on the compact $M$, such that

$$
\begin{aligned}
\left|\xi_{+}(\tau, t)-V^{+}\right| \mathrm{e}^{\frac{2 \pi \delta|\tau|}{p}} & \leq C \cdot\left\|\left(\xi_{+}(\tau, t)-V^{+}\right) \mathrm{e}^{\frac{2 \pi \delta|\tau|}{p}}\right\|_{W^{1, p}\left([L-1, L+1] \times S^{1}, u_{+}^{*}(T M)\right)} \\
& \leq C\left\|\xi_{+}\right\|_{1, p, \alpha} \quad(\text { see }(10.34)) \\
& \leq C\left\|Q_{+}\right\|\left\|\eta_{+}\right\|_{p, \alpha} \quad \text { (by Proposition 10.1). }
\end{aligned}
$$

Hence,

$$
\left|\xi_{+}(\tau, t)-V^{+}\right| \leq C \mathrm{e}^{\frac{-2 \pi \delta|\tau|}{p}}\left\|\eta_{+}\right\|_{p, \alpha}
$$

The following lemma concerns the commutativity of the operator $D \bar{\partial}$ with the operators $I^{S}$ and $J_{*, \varepsilon}^{S}$ :

Lemma 10.5. For any $\eta \in L_{\alpha_{\delta, \varepsilon}}^{p}\left(\Lambda^{0,1}\left(u_{\mathrm{app}}^{\varepsilon}\right)^{*} T M\right)$ and the corresponding $\xi_{\varepsilon,-}, \xi_{\varepsilon, 0}$ and $\xi_{\varepsilon,+}$ defined in (10.47), and $S=\frac{1}{4} R(\varepsilon)$, we have

$$
\begin{aligned}
& \|\left(D_{u_{\mathrm{app}}^{\varepsilon}} \bar{\partial} \circ I^{S}\right)\left(\xi_{\varepsilon,-}, \xi_{\varepsilon, 0}, \xi_{\varepsilon,+}\right) \\
& -\left(\left(J_{-, \varepsilon}^{S}+J_{0, \varepsilon}^{S}+J_{+, \varepsilon}^{S}\right) \circ D_{\left(u_{-}, u_{0}, u_{+}\right)} \bar{\partial}\left(\xi_{\varepsilon,-}, \xi_{\varepsilon, 0}, \xi_{\varepsilon,+}\right)\right) \|_{p, \alpha_{\delta, \varepsilon}} \\
\leq & C\left(e^{\frac{-4 \pi \delta S}{p}}+\operatorname{dist}\left(u_{\mathrm{app}}^{\varepsilon}, u_{-}^{\varepsilon}\right)+\operatorname{dist}\left(u_{\mathrm{app}}^{\varepsilon}, u_{+}^{\varepsilon}\right)+\operatorname{dist}\left(u_{\mathrm{app}}^{\varepsilon}, u_{0}^{\varepsilon}\right)\right)\|\eta\|_{p, \alpha_{\delta, \varepsilon}},
\end{aligned}
$$

where $C$ is a constant independent on $\delta$ and $\varepsilon$. 
Proof. There are three reasons why $I$ and $J$ do not commute with $D_{u_{\mathrm{app}}^{\varepsilon}} \bar{\partial}$ and $D_{\left(u_{-}, u_{0}, u_{+}\right)} \bar{\partial}$. One is that we use the parallel transport along the minimal geodesics from $u_{\text {app }}^{\varepsilon}(\tau, t)$ to $u_{0}^{\varepsilon}(\tau, t)$ and $u_{ \pm}^{\varepsilon}(\tau, t)$ and vice versa. The terms caused by parallel transport are estimated by the second, third and the forth terms of the right-hand side of the inequality.

The second reason is that on $u_{0}$ we use $D_{u_{0}} \bar{\partial}_{J_{p}}$ while on the $\varepsilon u_{0}$ portion of $u_{\text {app }}^{\varepsilon}$ we use $D_{\varepsilon u_{0}} \bar{\partial}_{J}$. The deviation of $J$ from $J_{p}$ is controlled by $\operatorname{dist}\left(\varepsilon u_{0}(\tau, t)\right.$, $p)$. The estimate of (10.63) on this part is similar to the $\bar{\partial} u_{\text {app }}^{\varepsilon}$ error estimate we have carried out in Subsection 10.2, which takes care of the deviation of $J$ from $J_{p}$, which we do not repeat. This contribution is of the order $C \mathrm{e}^{\frac{-4 \pi \delta S}{p}}\|\eta\|_{p, \alpha_{\delta, \varepsilon}}$, if we choose $\delta<p \min \left\{\frac{1}{3}, \frac{c_{0}}{2 p}\right\}$ in the beginning (see (10.31) for the relevant estimate).

The third and more essential point is that we have used the cut-off functions. We need to control the terms caused by $\chi_{ \pm R(\varepsilon) \pm S}^{ \pm}(\tau)\left(\mathrm{Pal}_{ \pm, \varepsilon}\left(\xi_{\varepsilon, \pm}\right)-\right.$ $\left.V^{ \pm}\right)$and $\chi_{ \pm R(\varepsilon) \pm S}^{ \pm}(\tau)\left(\mathrm{Pal}_{0, \varepsilon}\left(\xi_{\varepsilon, 0}\right)-V^{ \pm}\right)$. By the definition of the cut-off functions, these terms are supported in

$$
\pm[R(\varepsilon) \pm S-1, R(\varepsilon) \pm S+1] \times S^{1} \subset \mathbb{R} \times S^{1} .
$$

For $(\tau, t)$ in these regions, by Lemma 10.4, these terms are controlled by $C \mathrm{e}^{-4 \pi \delta S}\|\eta\|_{p, \alpha_{\delta, \varepsilon}}$. For example,

$$
\begin{aligned}
& \left|\chi_{R(\varepsilon)-S}^{+}(\tau)\left(\mathrm{Pal}_{+, \varepsilon}\left(\xi_{\varepsilon,+}(\tau, t)\right)-V^{+}\right)\right| \\
& \leq C \mathrm{e}^{\frac{-2 \pi \delta|\tau-2 R(\varepsilon)|}{p}}\left\|Q_{+} \circ J_{\varepsilon,+}^{S}\left(\chi_{R(\varepsilon)}^{+} \eta\right)\right\|_{p, \alpha} \\
& \leq C \mathrm{e}^{\frac{-2 \pi \delta \cdot(R(\varepsilon)+S)}{p}}\left\|Q_{+}\right\|\left\|J_{\varepsilon,+}^{S}\left(\chi_{R(\varepsilon)}^{+} \eta\right)\right\|_{p, \alpha} \\
& \leq C \mathrm{e}^{\frac{-2 \pi \delta \cdot(R(\varepsilon)+S)}{p}}\left\|Q_{+}\right\|\|\eta\|_{p, \alpha_{\delta, \varepsilon}},
\end{aligned}
$$

where in the last inequality we have used (10.50).

On the other hand, the weight $\alpha_{\delta, \varepsilon}$ on the support of $\chi_{R(\varepsilon)-S}^{+}{ }^{\prime}(\tau)$ is $\mathrm{e}^{2 \pi \delta|\tau|} \leq \mathrm{e}^{2 \pi \delta \cdot(R(\varepsilon)-S+1)}$. Therefore, the $L_{\alpha_{\delta, \varepsilon}}^{p}$ contribution from these terms is no more than

$$
C \mathrm{e}^{-2 \pi \delta(R(\varepsilon)+S)} \mathrm{e}^{2 \pi \delta(R(\varepsilon)-S+1)}\|\eta\|_{p, \alpha_{\delta, \varepsilon}} \leq C \mathrm{e}^{-4 \pi \delta S}\|\eta\|_{p, \alpha_{\delta, \varepsilon}} .
$$

The proposition follows.

With the above lemmas we can prove that $Q^{\varepsilon}$ is an approximate right inverse:

Proposition 10.2. For sufficiently small $\varepsilon>0$,

$$
\left\|\left(D_{u_{\mathrm{app}}^{\varepsilon}} \bar{\partial} \circ Q^{\varepsilon}\right) \eta-\eta\right\|_{p, \alpha_{\delta, \varepsilon}} \leq \frac{1}{2}\|\eta\|_{p, \alpha_{\delta, \varepsilon}}
$$

for all $\eta \in L_{\alpha_{\delta, \varepsilon}}^{p}\left(\Lambda^{0,1}\left(u_{\mathrm{app}}^{\varepsilon}\right)^{*} T M\right)$. 
Proof. From the definition of $Q^{\varepsilon}$ and Lemma 10.5, we have

$$
\begin{aligned}
\mid & \left(D_{u_{\mathrm{app}}^{\varepsilon}} \bar{\partial} \circ Q^{\varepsilon}\right) \eta-\eta \|_{p, \alpha_{\delta, \varepsilon}} \\
= & \left\|\left(D_{u_{\mathrm{app}}^{\varepsilon}} \bar{\partial} \circ I^{S}\right)\left(\xi_{\varepsilon,-}, \xi_{\varepsilon, 0}, \xi_{\varepsilon,+}\right)-\eta\right\|_{p, \alpha_{\delta, \varepsilon}} \\
\leq & \|\left(J_{-, \varepsilon}^{S}+J_{0, \varepsilon}^{S}+J_{+, \varepsilon}^{S}\right) \circ D_{\left(u_{-}, u_{0}, u_{+}\right)} \bar{\partial} \circ\left(Q_{-} J_{\varepsilon,-}^{S} \chi_{R(\varepsilon)}^{-} \eta, Q_{0} J_{\varepsilon, 0}^{S} \chi_{R(\varepsilon)}^{0} \eta,\right. \\
& \left.Q_{+} J_{\varepsilon,+}^{S} \chi_{R(\varepsilon)}^{+} \eta\right)-\eta\left\|_{p, \alpha_{\delta, \varepsilon}}+o(S)\right\| \eta \|_{p, \alpha_{\delta, \varepsilon}} \\
= & \left\|\left(J_{-, \varepsilon}^{S}+J_{0, \varepsilon}^{S}+J_{+, \varepsilon}^{S}\right) \circ\left(J_{\varepsilon,-}^{S} \chi_{R(\varepsilon)}^{-} \eta, J_{\varepsilon, 0}^{S} \chi_{R(\varepsilon)}^{0} \eta, J_{\varepsilon,+}^{S} \chi_{R(\varepsilon)}^{+} \eta\right)-\eta\right\|_{p, \alpha_{\delta, \varepsilon}} \\
& +o(S)\|\eta\|_{p, \alpha_{\delta, \varepsilon}} \\
= & \left\|\chi_{R(\varepsilon)}^{-} \eta+\chi_{R(\varepsilon)}^{0} \eta+\chi_{R(\varepsilon)}^{+} \eta-\eta\right\|_{p, \alpha_{\delta, \varepsilon}}+o(S)\|\eta\|_{p, \alpha_{\delta, \varepsilon}} \\
= & o(S)\|\eta\|_{p, \alpha_{\delta, \varepsilon},}
\end{aligned}
$$

where $o(S)$ is a term going to 0 when $S \rightarrow \infty$, and the second-to-last identity is due to $(10.43)$ and $\chi_{R(\varepsilon)}^{-}+\chi_{R(\varepsilon)}^{0}+\chi_{R(\varepsilon)}^{+}=1$.

When $\varepsilon$ is sufficiently small, $S=\frac{1}{4} R(\varepsilon)$ is very large and we get

$$
\left\|\left(D_{u_{\mathrm{app}}^{\varepsilon}} \bar{\partial} \circ Q^{\varepsilon}\right) \eta-\eta\right\|_{p, \alpha_{\delta, \varepsilon}} \leq \frac{1}{2}\|\eta\|_{p, \alpha_{\delta, \varepsilon}} .
$$

By (10.65), $D_{u_{\mathrm{app}}^{\varepsilon}} \bar{\partial} \circ Q^{\varepsilon}$ is invertible, and

$$
\left\|\left(D_{u_{\mathrm{app}}^{\varepsilon}} \bar{\partial} \circ Q^{\varepsilon}\right)^{-1}\right\|=\left\|\Sigma_{k=0}^{\infty}\left(D_{u_{\mathrm{app}}^{\varepsilon}} \bar{\partial} \circ Q^{\varepsilon}-\mathrm{i} d\right)^{k}\right\| \leq \Sigma_{k=0}^{\infty} \frac{1}{2^{k}}=1 .
$$

So we can construct the true right inverse for $D_{u_{\mathrm{app}}^{\varepsilon}} \bar{\partial}$ to be $Q^{\varepsilon} \circ\left(D_{u_{\mathrm{app}}^{\varepsilon}} \bar{\partial} \circ\right.$ $\left.Q^{\varepsilon}\right)^{-1}$. For convenience we still denote it by $Q^{\varepsilon}$. From its construction and Proposition 10.1 we see $\left\|Q^{\varepsilon}\right\|$ is bounded by a uniform constant $C$ for all $\varepsilon>0$.

Lemma 10.6. For all $\varepsilon>0$, and $\xi \in T_{u_{\mathrm{app}}^{\varepsilon}} \mathcal{B}_{\mathrm{res}}^{\varepsilon}\left(z_{-}, z_{+}\right)$, we have the uniform Sobolev inequality

$$
|\xi|_{\infty} \leq C_{p}\|\xi\|_{1, p, \alpha_{\delta, \varepsilon}},
$$

where the constant $C_{p}$ is independent on $\varepsilon$.

Proof. Since the base variation term $|v|$ appears on both sides of the above inequality, we can assume $v=0$ for $\xi$. For the maps $u_{\text {app }}^{\varepsilon}: \mathbb{R} \times S^{1} \rightarrow$ $\left(M, g_{\varepsilon_{0}, \varepsilon, p}\right)$, the domain $\mathbb{R} \times S^{1}$ with standard metric is noncollapsing; The targets $\left(M, g_{\varepsilon_{0}, \varepsilon, p}\right)$ for all $0<\varepsilon<\varepsilon_{0}$ and $p \in M$ is a family of noncollasping Riemannian manifolds. Therefore, the Sobolev constant

$$
c_{p}\left(u_{\mathrm{app}}^{\varepsilon}\right):=\sup _{0 \neq \xi \in \Gamma\left(\left(u_{\mathrm{app}}^{\varepsilon}\right) * T M\right)} \frac{|\xi|_{L^{\infty}}}{\|\xi\|_{W^{1, p}}}
$$

is uniformly bounded above for all $\varepsilon$, where $\Gamma\left(\left(u_{\mathrm{app}}^{\varepsilon}\right)^{*} T M\right)$ is the set of all $C_{0}^{\infty}$ sections of $\left(u_{\mathrm{app}}^{\varepsilon}\right)^{*} T M$. So we have a uniform constant $c_{p}$ such that 
$|\cdot|_{L^{\infty}} \leq c_{p}\|\cdot\|_{1, p}$. Certainly this is still true if we change the $W^{1, p}$ norm to positive exponential weighted norm: $|\cdot|_{L^{\infty}} \leq c_{p}\|\cdot\|_{1, p, \delta}$, where the weight $\delta(\tau, t)$ is $\mathrm{e}^{2 \pi \delta|\tau|}$ on $\mathbb{R} \times S^{1}$.

However, our norm $\|\cdot\|_{1, p, \alpha_{\delta, \varepsilon}}$ is not the usual weighted Sobolev norm, because we first take out the "Morse-Bott variation" of $\xi$ and then measure the remaining part by the weighted Sobolev norm. To get the Sobolev inequality, notice that

$$
|\xi(\tau, t)| \leq\left|\xi-\beta_{\varepsilon}^{ \pm}(\tau) \mathrm{Pal}_{u_{\mathrm{app}}^{\varepsilon}(\tau, t)} \mathrm{Pal}_{u_{\mathrm{app}}^{\varepsilon}( \pm R(\varepsilon), t)} \xi( \pm R(\varepsilon), 0)\right|+|\xi( \pm R(\varepsilon), 0)| .
$$

Apply $|\cdot|_{L^{\infty}} \leq c_{p}\|\cdot\|_{1, p, \delta}$ to the first term on the right-hand side of the above inequality, and recall the definition of the norm $\|\cdot\|_{1, p, \alpha_{\delta, \varepsilon}}$, then we get

$$
|\xi|_{\infty} \leq \max \left\{c_{p}, 1\right\}\|\xi\|_{1, p, \alpha_{\delta, \varepsilon}} .
$$

Letting $C_{p}=\max \left\{c_{p}, 1\right\}$, the lemma follows.

Proposition 10.3. For every $u_{\mathrm{app}}^{\varepsilon} \in \mathcal{B}_{\mathrm{res}}^{\varepsilon}\left(z_{-}, z_{+}\right)$, there exist constants $h_{\varepsilon}=K e^{-2 \pi \delta R(\varepsilon)}$, where the constant $K$ is independent on $\varepsilon$, such that for every $0<\varepsilon \leq \varepsilon_{0}$, and every $\xi \in T_{u_{\mathrm{app}}^{\varepsilon}}\left(\mathcal{B}_{\mathrm{res}}^{\varepsilon}\left(z_{-}, z_{+}\right)\right)$with $\|\xi\|_{1, p, \alpha_{\delta, \varepsilon}} \leq h_{\varepsilon}$, we have

$$
\left\|d \mathcal{F}_{u_{\mathrm{app}}^{\varepsilon}}(\xi) \xi^{\prime}-\left(D_{u_{\mathrm{app}}^{\varepsilon}} \bar{\partial}_{\left(J_{\varepsilon}, K_{\varepsilon}\right)}\right) \xi^{\prime}\right\|_{p, \alpha_{\delta, \varepsilon}} \leq \frac{1}{2 C}\left\|\xi^{\prime}\right\|_{1, p, \alpha_{\delta, \varepsilon}}
$$

for all $\xi^{\prime} \in T_{u_{\mathrm{app}}^{\varepsilon}}\left(\mathcal{B}_{\mathrm{res}}^{\varepsilon}\left(z_{-}, z_{+}\right)\right)$. Here $C$ is the uniform bound for $\left\|Q^{\varepsilon}\right\|$.

Proof. The proof is a variation of the Proposition 3.5.3 in [MS]. The point estimate is the same as $[\mathbf{M S}]$. The main differences are that our norm $\|\cdot\|_{1, p, \alpha_{\delta, \varepsilon}}$ is not the usual $W^{1, p}$ norm in [MS], and our target manifold $\left(M, g_{\varepsilon_{0}, \varepsilon, p}\right)$ is stretching when $\varepsilon \rightarrow 0$.

Let $\left.\mathcal{F}_{u_{\mathrm{app}}^{\varepsilon}}: T_{u_{\mathrm{app}}^{\varepsilon}} \mathcal{B}_{\mathrm{res}}^{\varepsilon}\left(z_{-}, z_{+}\right) \rightarrow L_{\alpha_{\delta, \varepsilon}}^{p}\left(\Lambda^{0,1}\left(u_{\mathrm{app}}^{\varepsilon}\right)^{*} T M\right)\right)$ be

$$
\mathcal{F}_{u_{\mathrm{app}}^{\varepsilon}}(\xi)=\left(\Phi_{u_{\mathrm{app}}^{\varepsilon}}(\xi)\right)^{-1}\left(\bar{\partial}_{\left(J_{\varepsilon, K_{\varepsilon}}\right)}\left(\exp _{u_{\mathrm{app}}^{\varepsilon}} \xi\right)\right),
$$

where $\Phi_{u_{\mathrm{app}}^{\varepsilon}}(\xi):\left(u_{\mathrm{app}}^{\varepsilon}\right)^{*} T M \rightarrow\left(\exp _{u_{\mathrm{app}}^{\varepsilon}}(\xi)\right)^{*} T M$ is the parallel transport in $\left(M, g_{\varepsilon_{0}, \varepsilon, p}\right)$ along the geodesics $s \rightarrow \exp _{u_{\mathrm{app}}^{\varepsilon}(z)}(s \xi(z))$. Then the differential of $\mathcal{F}_{u_{\mathrm{app}}^{\varepsilon}}$ satisfies $\mathcal{F}_{u_{\mathrm{app}}^{\varepsilon}}(0)=D_{u_{\mathrm{app}}^{\varepsilon}} \bar{\partial}_{\left(J_{\varepsilon}, K_{\varepsilon}\right)}$.

For each $\varepsilon>0,\left(M, g_{\varepsilon_{0}, \varepsilon, p}\right)$ is a compact Riemannian manifold. The point estimate in the proof of Proposition 3.5.3 in [MS] yields

$$
\left|d \mathcal{F}_{u_{\mathrm{app}}^{\varepsilon}}(\xi) \xi^{\prime}-D_{u_{\mathrm{app}}^{\varepsilon}} \bar{\partial}_{\left(J_{\varepsilon}, K_{\varepsilon}\right)} \xi^{\prime}\right| \leq A\left(|d u||\xi|\left|\xi^{\prime}\right|+|\nabla \xi|\left|\xi^{\prime}\right|+|\xi|\left|\nabla \xi^{\prime}\right|\right) .
$$

Here the constant $A>0$ is determined by the Sobolev constant $C_{p}$, hence $A$ is uniform for all $\varepsilon>0$.

By our construction of $u_{\text {app }}^{\varepsilon}$, there exists a uniform constant $B$ for all $\varepsilon>0$, such that $\left|d u_{\text {app }}^{\varepsilon}(\tau, t)\right|_{g_{\varepsilon_{0}, \varepsilon, p}} \leq B$ for all $(\tau, t) \in \mathbb{R} \times S^{1}$. We consider three cases for $\xi$ : 
Case 1: $\xi( \pm R(\varepsilon), 0)=0$, i.e. there is no Morse-Bott variation. In this case, $\|\xi\|_{1, p, \alpha_{\delta, \varepsilon}}$ is the usual weighted Sobolev norm. Multiplying $\mathrm{e}^{\frac{2 \pi \delta|\tau|}{p}}$ to both sides of (10.68) and taking the $L^{p}$ integration over $\mathbb{R} \times S^{1}$, we get

$$
\begin{aligned}
& \left\|d \mathcal{F}_{u_{\mathrm{app}}^{\varepsilon}}(\xi) \xi^{\prime}-D_{u_{\mathrm{app}}^{\varepsilon}} \bar{\partial}_{\left(J_{\varepsilon}, K_{\varepsilon}\right)} \xi^{\prime}\right\|_{p, \alpha_{\delta, \varepsilon}} \\
& \leq A\left(B\|\xi\|_{1, p, \alpha_{\delta, \varepsilon}}\left|\xi^{\prime}\right|_{\infty}+\|\xi\|_{1, p, \alpha_{\delta, \varepsilon}}\left|\xi^{\prime}\right|_{\infty}+|\xi|_{\infty}\left\|\xi^{\prime}\right\|_{p, \alpha_{\delta, \varepsilon}}\right) \\
& \leq A\left(B+2 C_{p}\right)\|\xi\|_{1, p, \alpha_{\delta, \varepsilon}} \cdot C_{p}\left\|\xi^{\prime}\right\|_{p, \alpha_{\delta, \varepsilon}},
\end{aligned}
$$

where in the last inequality we have used the Sobolev inequality (10.66). The proposition is proved by taking $h_{\varepsilon}=\left(A C_{p}\left(B+2 C_{p}\right)\right)^{-1} \frac{1}{2 C}$.

Case 2: $\xi(\tau, t)=\beta_{\varepsilon}^{ \pm}(\tau) \mathrm{Pal}_{u_{\mathrm{app}}^{\varepsilon}(\tau, t)} \mathrm{Pal}_{u_{\mathrm{app}}^{\varepsilon}( \pm R(\varepsilon), t)} \xi( \pm R(\varepsilon), 0)$, i.e., $\xi$ is purely induced from the Morse-Bott variation. In this case, $\|\xi\|_{1, p, \alpha_{\delta, \varepsilon}}=$ $|\xi(R(\varepsilon), 0)|$, and by construction $|\xi|_{\infty} \leq\|\xi\|_{1, p, \alpha_{\delta, \varepsilon}}$. We also have

$$
\begin{aligned}
|\nabla \xi|_{\infty} & =\left|\nabla\left(\beta_{\varepsilon}^{ \pm}(\tau) \mathrm{Pal}_{u_{\mathrm{app}}^{\varepsilon}(\tau, t)} \mathrm{Pal}_{u_{\mathrm{app}}^{\varepsilon}( \pm R(\varepsilon), t)} \xi( \pm R(\varepsilon), 0)\right)\right|_{\infty} \\
& \leq|\xi( \pm R(\varepsilon), 0)|+\left|d u_{\mathrm{app}}^{\varepsilon}\right|_{\infty} \cdot|\xi( \pm R(\varepsilon), 0)| \\
& \leq(1+B)|\xi( \pm R(\varepsilon), 0)| \\
& =(1+B)\|\xi\|_{1, p, \alpha_{\delta, \varepsilon}} .
\end{aligned}
$$

Therefore at any $(\tau, t)$,

$$
\begin{aligned}
& |d u||\xi|\left|\xi^{\prime}\right|+|\nabla \xi|\left|\xi^{\prime}\right|+|\xi|\left|\nabla \xi^{\prime}\right| \\
& \leq B\|\xi\|_{1, p, \alpha_{\delta, \varepsilon}}\left|\xi^{\prime}\right|_{\infty}+(1+B)\|\xi\|_{1, p, \alpha_{\delta, \varepsilon}}\left|\xi^{\prime}\right|_{\infty}+\|\xi\|_{1, p, \alpha_{\delta, \varepsilon}}\left|\nabla \xi^{\prime}\right| .
\end{aligned}
$$

Multiplying $\mathrm{e}^{\frac{2 \pi \delta|\tau|}{p}}$ to both sides of the above inequality and taking the $L^{p}$ integration over $\mathbb{R} \times S^{1}$, and noticing that $\xi$ is supported in $\tau \in[0, \pm 2 R(\varepsilon)]$, we get

$$
\begin{aligned}
\| & d \mathcal{F}_{u_{\mathrm{app}}^{\varepsilon}}(\xi) \xi^{\prime}-D_{u_{\mathrm{app}}^{\varepsilon}} \bar{\partial}_{\left(J_{\varepsilon}, K_{\varepsilon}\right)} \xi^{\prime} \|_{p, \alpha_{\delta, \varepsilon}} \\
\leq & \left.A(1+2 B)\|\xi\|_{1, p, \alpha_{\delta, \varepsilon}} \xi^{\prime}\right|_{\infty} \cdot\left(\int_{[0, \pm 2 R(\varepsilon)] \times S^{1}} \mathrm{e}^{2 \pi \delta|\tau|} d \tau d t\right)^{\frac{1}{p}} \\
+ & A\|\xi\|_{1, p, \alpha_{\delta, \varepsilon}}\left(\int_{\mathbb{R} \times S^{1}} \mathrm{e}^{2 \pi \delta|\tau|}\left|\nabla \xi^{\prime}\right| d \tau d t\right)^{\frac{1}{p}} \\
\leq & A(1+2 B)\|\xi\|_{1, p, \alpha_{\delta, \varepsilon}} \cdot C_{p}\left\|\xi^{\prime}\right\|_{1, p, \alpha_{\delta, \varepsilon}} \cdot\left(\frac{1}{2 \pi \delta}\right)^{\frac{1}{p}} \cdot \mathrm{e}^{\frac{4 \pi \delta R(\varepsilon)}{p}} \\
& +A\|\xi\|_{1, p, \alpha_{\delta, \varepsilon}}\left\|\xi^{\prime}\right\|_{1, p, \alpha_{\delta, \varepsilon}} \\
= & A\left(\frac{(1+2 B) C_{p}}{(2 \pi \delta)^{\frac{4 \pi \delta R(\varepsilon)}{p}}} \mathrm{e}^{\frac{4 \pi \delta(\varepsilon)}{p}}+1\right)\|\xi\|_{1, p, \alpha_{\delta, \varepsilon}}\left\|\xi^{\prime}\right\|_{1, p, \alpha_{\delta, \varepsilon}} \\
\leq & K\left(A, B, \delta, C_{p}\right) \mathrm{e}^{\frac{4 \pi \delta R(\varepsilon)}{p}}\|\xi\|_{1, p, \alpha_{\delta, \varepsilon}}\left\|\xi^{\prime}\right\|_{1, p, \alpha_{\delta, \varepsilon}},
\end{aligned}
$$


where $K\left(A, B, \delta, C_{p}\right)$ is a constant independent on $\varepsilon$. Therefore, the proposition is proved by taking $h_{\varepsilon}=\left(K\left(A, B, \delta, C_{p}\right)\right)^{-1} \frac{1}{2 C} \cdot \mathrm{e}^{-\frac{4 \pi \delta R(\varepsilon)}{p}}$.

Case 3: For general $\xi$. We can write $\xi=\xi_{1}+\xi_{2}$, where $\xi_{1}$ is in case 1 and $\xi_{2}$ is in case 2 . Then we can apply triangle inequality on the terms involving $\xi$ in the point estimate (10.68), and then $L^{p}$ integrate the point estimate. The proof reduces to cases 1 and 2 .

Combining the three cases, there exists a constant $K$ independent on $\varepsilon$, such that for $h_{\varepsilon}:=K \mathrm{e}^{-\frac{4 \pi \delta R(\varepsilon)}{p}}$ and $\|\xi\|_{1, p, \alpha_{\delta, \varepsilon}} \leq h_{\varepsilon}$,

$$
\left\|d \mathcal{F}_{u_{\mathrm{app}}^{\varepsilon}}(\xi) \xi^{\prime}-\left(D_{u_{\mathrm{app}}^{\varepsilon}} \bar{\partial}_{\left(J_{\varepsilon}, K_{\varepsilon}\right)}\right) \xi^{\prime}\right\|_{p, \alpha_{\delta, \varepsilon}} \leq \frac{1}{2 C}\left\|\xi^{\prime}\right\|_{1, p, \alpha_{\delta, \varepsilon}} .
$$

Remark 10.7. In our setting, for each $\varepsilon$, the almost complex structure $J_{\varepsilon}$ is $(\tau, t)$-dependent, while in [MS] it is not. But since $J(\tau, t) \equiv J_{0}$ for $\tau \in$ $[-R(\varepsilon), R(\varepsilon)]$, and $J(\tau, t) \equiv J(t)$ for $|\tau|>R(\varepsilon)+1$, our $J_{\varepsilon}(\tau, t)$ is actually a compact family of almost complex structures (smoothly parameterized by $\left.\pm[R(\varepsilon), R(\varepsilon)+1] \times S^{1}\right)$. Therefore, the proof in the compact family of $J_{\varepsilon}(\tau, t)$ case is the same as the fixed $J$ case (see the Remark 3.5.4 in [MS]).

Remark 10.8. Unlike [MS], in our case $\left\|d u_{\text {app }}^{\varepsilon}\right\|_{p, \alpha_{\delta, \varepsilon}} \rightarrow \infty$, and we only have $\left|d u_{\text {app }}^{\varepsilon}\right|_{\infty} \leq B$. In the case $2,|\xi|_{p, \alpha_{\delta, \varepsilon}} \rightarrow \infty$, and we only have $|\xi|_{\infty} \leq$ $|\xi(R(\varepsilon), 0)|$. The loss of the exponential decay of $d u_{\text {app }}^{\varepsilon}$ and $\xi$ is caused by the stretching of the target manifold $\left(M, g_{\varepsilon_{0}, \varepsilon, p}\right)$ when $\varepsilon \rightarrow 0$. This is the reason that our estimate $(10.67)$ is weaker than that in [MS], where the latter is on a fixed compact Riemannian manifold.

For gluing we need the following abstract implicit function theorem in [MS]:

Proposition 10.4. Let $X, Y$ be Banach spaces and $U$ be an open set in $X$. The map $f: X \rightarrow Y$ is continuous differentiable. For $\xi_{0} \in U, D:=d f\left(x_{0}\right)$ : $X \rightarrow Y$ is surjective and has a bounded linear right inverse $Q: Y \rightarrow X$, with $\|Q\| \leq C$. Suppose that there exists $h>0$ such that for all $x \in B_{h}\left(x_{0}\right) \subset U$,

$$
\|d f(x)-D\| \leq \frac{1}{2 C} .
$$

Then for $\left\|f\left(x_{0}\right)\right\| \leq \frac{h}{4 C}$, there exists a unique $x \in B_{h}\left(x_{0}\right)$ such that

$$
f(x)=0, \quad x-x_{0} \in \text { Image } Q, \quad\left\|x-x_{0}\right\| \leq 2 C\left\|f\left(x_{0}\right)\right\| .
$$

Now we apply the above implicit function theorem in the following setting: $X=T_{u_{\mathrm{app}}^{\varepsilon}} \mathcal{B}_{\mathrm{res}}^{\varepsilon}\left(z_{-}, z_{+}\right), \quad Y=L_{\alpha_{\delta, \varepsilon}}^{p}\left(\Lambda^{0,1}\left(u_{\mathrm{app}}^{\varepsilon}\right)^{*} T M\right), \quad f=\mathcal{F}_{u_{\mathrm{app}}^{\varepsilon}}, \quad x_{0}=0$. Then from Proposition 10.3 we have

$$
\left\|d \mathcal{F}_{u_{\mathrm{app}}^{\varepsilon}}(\xi)-d \mathcal{F}_{u_{\mathrm{app}}^{\varepsilon}}(0)\right\| \leq \frac{1}{2 C}
$$


for $\xi$ in $X$ with $\|\xi\|_{1, p, \alpha_{\delta, \varepsilon}} \leq h_{\varepsilon}:=K \mathrm{e}^{-\frac{4 \pi \delta R(\varepsilon)}{p}}$. From (10.33), we have

$$
\left\|\mathcal{F}_{u_{\text {app }}^{\varepsilon}}(0)\right\| \leq L \cdot(R(\varepsilon))^{\frac{1}{p}} \cdot \mathrm{e}^{-\frac{4 \pi a R(\varepsilon)}{p}},
$$

hence for $\varepsilon$ small,

$$
\left\|\mathcal{F}_{u_{\mathrm{app}}^{\varepsilon}}(0)\right\| \leq \frac{1}{4 C} \cdot K e^{-\frac{4 \pi \delta R(\varepsilon)}{p}}=\frac{h_{\varepsilon}}{4 C} .
$$

Here we have used our choice of $0<\delta<a$ in the beginning, so ( $R$ $(\varepsilon))^{\frac{1}{p}} \cdot \mathrm{e}^{-\frac{4 \pi a R(\varepsilon)}{p}}$ decays faster than $\mathrm{e}^{-\frac{4 \pi \delta R(\varepsilon)}{p}}$. By the above abstract implicit function theorem, we have finished the gluing and prove the following theorem, which is a half of Theorem 5.2.

Theorem 10.1. Let $\left(K_{\varepsilon}, J_{\varepsilon}\right)$ be the family of Floer data defined in (5.34). Then

(1) There exists a topology on $\mathcal{M}_{(0 ; 1,1)}^{\text {para }}\left(\left[z_{-}, w_{-}\right],\left[z_{+}, w_{+}\right]\right) ;\left\{\left(K_{\varepsilon}, J_{\varepsilon}\right)\right\}$ with respect to which the gluing construction defines a proper embedding

$$
\begin{aligned}
\text { Glue } & :\left(0, \varepsilon_{0}\right) \times \mathcal{M}_{(0 ; 1,1)}^{\text {nodal }}\left(\left[z_{-}, w_{-}\right],\left[z_{+}, w_{+}\right] ;(H, J),\left(f, J_{0}\right)\right) \\
& \left.\rightarrow \mathcal{M}_{(0 ; 1,1)}^{\text {para }}\left(\left[z_{-}, w_{-}\right],\left[z_{+}, w_{+}\right]\right) ;\{(K, J)\}\right)
\end{aligned}
$$

for sufficiently small $\varepsilon_{0}$.

(2) The above mentioned topology can be compactified into

$$
\left.\overline{\mathcal{M}}_{(0 ; 1,1)}^{\text {para }}\left(\left[z_{-}, w_{-}\right],\left[z_{+}, w_{+}\right]\right) ;\{(K, J)\}\right)
$$

where $\left.\overline{\mathcal{M}}_{(0 ; 1,1)}^{\text {para }}\left(\left[z_{-}, w_{-}\right],\left[z_{+}, w_{+}\right]\right) ;\{(K, J)\}\right)$ is given by

$$
\begin{aligned}
& \left.\overline{\mathcal{M}}_{(0 ; 1,1)}^{\text {para }}\left(\left[z_{-}, w_{-}\right],\left[z_{+}, w_{+}\right]\right) ;\{(K, J)\}\right) \\
& =\bigcup_{0<\varepsilon \leq \varepsilon_{0}} \mathcal{M}_{(0 ; 1,1)}\left(\left[z_{-}, w_{-}\right],\left[z_{+}, w_{+}\right]\right) ;\left\{\left(K_{\varepsilon}, J_{\varepsilon}\right)\right\} \\
& \quad \cup \mathcal{M}_{(0 ; 1,1)}^{\text {nodal }}\left(\left[z_{-}, w_{-}\right],\left[z_{+}, w_{+}\right] ;(H, J),\left(f, J_{0}\right)\right)
\end{aligned}
$$

as a set,

(3) The embedding Glue smoothly extends to the embedding

$$
\begin{aligned}
\overline{\text { Glue }}: & {\left[0, \varepsilon_{0}\right) \times \mathcal{M}_{(0 ; 1,1)}^{\text {nodal }}\left(\left[z_{-}, w_{-}\right],\left[z_{+}, w_{+}\right] ;(H, J),\left(f, J_{0}\right)\right) } \\
& \left.\rightarrow \overline{\mathcal{M}}_{(0 ; 1,1)}^{\text {para }}\left(\left[z_{-}, w_{-}\right],\left[z_{+}, w_{+}\right]\right) ;\{(K, J)\}\right)
\end{aligned}
$$

that satisfies

$$
\overline{\operatorname{Glue}}\left(u_{+}, u_{-}, u_{0} ; 0\right)=\operatorname{Glue}\left(u_{+}, u_{-}, u_{0}\right) .
$$

For $0<\varepsilon \leq \varepsilon_{0}$, we denote by

$$
\operatorname{Glue}\left(u_{+}, u_{-}, u_{0} ; \varepsilon\right) \in \mathcal{M}_{(0 ; 1,1)}^{\mathrm{para}}\left(\left[z_{-}, w_{-}\right],\left[z_{+}, w_{+}\right]\right) ;\left\{\left(K_{\varepsilon}, J_{\varepsilon}\right)\right.
$$


the image of

$$
\left(u_{+}, u_{-}, u_{0} ; \varepsilon\right) \in\left[0, \varepsilon_{0}\right) \times \mathcal{M}_{(0 ; 1,1)}^{\text {nodal }}\left(\left[z_{-}, w_{-}\right],\left[z_{+}, w_{+}\right] ;(H, J),\left(f, J_{0}\right)\right)
$$

under the embedding Glue.

In the rest of part II of the paper, we will prove that $\overline{\text { Glue }}$ is surjective onto an open neighborhood of $\mathcal{M}_{(0 ; 1,1)}^{\text {nodal }}\left(\left[z_{-}, w_{-}\right],\left[z_{+}, w_{+}\right] ;(H, J),\left(f, J_{0}\right)\right)$ in

$$
\left.\overline{\mathcal{M}}_{(0 ; 1,1)}^{\text {para }}\left(\left[z_{-}, w_{-}\right],\left[z_{+}, w_{+}\right]\right) ;\{(K, J)\}\right) .
$$

\section{Adiabatic degeneration: analysis of the thin part}

In this section, we consider a one-parameter family $\left(K_{\varepsilon}, J_{\varepsilon}\right)$ as provided in Subsection 5.5 with $R=R(\varepsilon) \rightarrow \infty$,

$$
\varepsilon R(\varepsilon) \rightarrow \ell
$$

with $\ell \geq 0$ as $\varepsilon \rightarrow 0$. Motivated by the gluing construction in the previous section, we will be particularly interested in the case where $\ell=0$, e.g.,

$$
R(\varepsilon)=-\frac{\log \varepsilon}{4 \pi} .
$$

We recall $\delta_{\varepsilon}$ satisfies

$$
\delta_{\varepsilon} / \varepsilon \rightarrow \infty \quad \text { as } \varepsilon \rightarrow 0 .
$$

We use the Hamiltonian defined by

$$
K_{\varepsilon}(\tau, t)= \begin{cases}\kappa_{\varepsilon}^{+}(\tau) \cdot H_{t}, & \tau \geq R(\varepsilon), \\ \rho_{\varepsilon}(\tau) \cdot \varepsilon f, & |\tau| \leq R(\varepsilon), \\ \kappa_{\varepsilon}^{-}(\tau) \cdot H_{t}, & \tau \leq-R(\varepsilon) .\end{cases}
$$

The $K_{\varepsilon}(\tau, t)$ was defined before in (5.34), where $\kappa_{\varepsilon}^{+}(\tau)$ was defined in (5.19) and $\rho_{\varepsilon}(\tau)$ was defined in (5.30). We then study the family of equation

$$
\left(d u+P_{K_{R(\varepsilon)}}(u)\right)_{J}^{(0,1)}=0
$$

as $\varepsilon \rightarrow 0$. For the simplicity of notation, we denote $K_{\varepsilon}(\tau, t, x)=K_{\varepsilon}$. By definition of $K_{\varepsilon}$ and $J_{\varepsilon}$, as $\varepsilon \rightarrow 0$, on the domain

$$
[-R(\varepsilon)+1, R(\varepsilon)-1] \times S^{1},
$$

we have $K_{\varepsilon}(\tau, t) \equiv \varepsilon f$ and $J_{R}(\tau, t) \equiv J_{0}$, and so (11.4) becomes

$$
\frac{\partial u}{\partial \tau}+J_{0}\left(\frac{\partial u}{\partial t}-\varepsilon X_{f}(u)\right)=0
$$

Furthermore, $K_{\varepsilon}(\tau, t) \equiv H_{t} d t, J_{R}(\tau, t) \equiv J_{t}$ on

$$
\mathbb{R} \times S^{1} \backslash[-R(\varepsilon)+1, R(\varepsilon)-1] \times S^{1} .
$$

Equation (11.4) is cylindrical at infinity, i.e., invariant under the translation in $\tau$-direction at infinity. 
Note that on any fixed compact set $B \subset \mathbb{R} \times S^{1}$, we will have

$$
B \subset[-R(\varepsilon), R(\varepsilon)] \times S^{1}
$$

for all sufficiently small $\varepsilon$. And as $\varepsilon \rightarrow 0, K_{\varepsilon} \rightarrow 0$ on $B$ in $C^{\infty}$-topology, and hence equation (11.4) converges to $\bar{\partial}_{J_{0}} u=0$ on $B$ in that $J \rightarrow J_{0}$ and $K_{\varepsilon} \rightarrow 0$ in $C^{\infty}$-topology. On the other hand, after translating the region $\left(-\infty,-\left(R-\frac{1}{3}\right]\right.$ to the right (resp. $\left[R-\frac{1}{3}, \infty\right)$ to the left) by $2 R-\frac{2}{3}$ in $\tau$-direction, (11.4) converges to

$$
\frac{\partial u}{\partial \tau}+J_{0}\left(\frac{\partial u}{\partial t}-X_{H}(u)\right)=0
$$

on $(-\infty, 0] \times S^{1}$ (resp. on $\left.[0, \infty) \times S^{1}\right)$ and $\bar{\partial}_{J_{0}} u=0$ on $\left[0, R-\frac{1}{3}\right] \times S^{1}$ (resp. on $\left.\left[-R+\frac{1}{3}, 0\right] \times S^{1}\right)$.

Now we are ready to state the meaning of the level-0 convergence for a sequence $u_{n}$ of solutions $\left(d u+P_{K_{\varepsilon_{n}}}\right)_{J_{\varepsilon_{n}}}^{(0,1)}=0$ as $n \rightarrow \infty$. After taking away bubbles, we assume that we have the derivative bound

$$
|d u|<C<\infty
$$

where we take the norm $|d u|$ with respect to the given metric $g$ on $M$. We denote

$$
\Theta_{\varepsilon}=\left[-R(\varepsilon)+\frac{1}{3}, R(\varepsilon)-\frac{1}{3}\right] \times S^{1}
$$

and consider the local energy

$$
E_{J, \Theta_{\varepsilon}}(u)=\int_{-R(\varepsilon)+1 / 3}^{R(\varepsilon)-1 / 3} \int_{S^{1}}|d u|_{J}^{2} d t d \tau .
$$

There are two cases to consider:

(1) there exists $c>0$ such that $E_{J, \Theta_{\varepsilon_{n}}}\left(u_{n}\right)>c>0$ for all sufficiently large $n$

(2) $\lim _{n \rightarrow \infty} E_{J, \Theta_{\varepsilon_{n}}}\left(u_{n}\right)=0$.

For the case (1), standard argument produces a nonconstant bubble and so we will mainly consider the case (2). Therefore from now on, we will assume

$$
\lim _{j \rightarrow \infty} E_{J, \Theta_{\varepsilon_{j}}}\left(u_{j}\right)=0 .
$$

Now we consider the reparameterization

$$
\bar{u}_{j}(\tau, t)=u_{j}\left(\frac{\tau}{\varepsilon_{j}}, \frac{t}{\varepsilon_{j}}\right)
$$

on the domain $\left[-\varepsilon_{j} R\left(\varepsilon_{j}\right), \varepsilon_{j} R\left(\varepsilon_{j}\right)\right] \times \mathbb{R} / 2 \pi \varepsilon_{j} \mathbb{Z}$. A straightforward calculation shows that $\bar{u}_{j}$ satisfies

$$
\frac{\partial \bar{u}}{\partial \tau}+J_{0}\left(\frac{\partial \bar{u}}{\partial t}-X_{f}(u)\right)=0
$$


or equivalently

$$
\frac{\partial \bar{u}}{\partial \tau}+J_{0} \frac{\partial \bar{u}}{\partial t}+\operatorname{grad}_{J_{0}} f(u)=0
$$

on $\left[-\varepsilon_{j} R\left(\varepsilon_{j}\right), \varepsilon_{j} R\left(\varepsilon_{j}\right)\right] \times \mathbb{R} / 2 \pi \varepsilon_{j} \mathbb{Z}$. For the simplicity of notation, we will sometimes denote

$$
R_{j}=R\left(\varepsilon_{j}\right) .
$$

The following result was proved in Part II of [Oh3]. A similar result was also obtained by Mundet i Riera and Tian. (See Theorem 1.3 [MT].)

Theorem 11.1 ( $[\mathrm{Oh} 3, \mathrm{MT}]$ ). Suppose

$$
\ell=\lim _{j \rightarrow \infty} \varepsilon_{j} R\left(\varepsilon_{j}\right), \quad \lim _{j \rightarrow \infty} E_{J, \Theta_{R_{j}}}\left(u_{j}\right)=0 .
$$

Then there exists a subsequence, again denoted by $u_{j}$, such that the reparameterized map $\bar{u}_{j}$ converges to a $\chi:[-\ell, \ell] \rightarrow M$ satisfying $\dot{\chi}+\operatorname{grad}_{J} f(\chi)=0$ in $C^{\infty}$-topology. In particular, when $\ell=0$, the original map $\left.u_{j}\right|_{\Theta_{\varepsilon_{j}}}$ converges to a point $p \in M$.

Under this assumption $\lim _{\varepsilon \rightarrow 0} E_{J, \Theta_{R}}\left(u_{j}\right)=0$, after taking away bubbles, the translated sequences $\left.u_{j}\left(\cdot \pm R_{j}+1\right), \cdot\right):\left(-\infty, R_{j}\right] \times S^{1} \rightarrow M$ of solutions $u_{j}$ of (11.4) as above converge to $u_{-}, u_{+}: \mathbb{R} \times S^{1} \rightarrow M$ that satisfies the equation

$$
\frac{\partial u}{\partial \tau}+J_{0}\left(\frac{\partial u}{\partial t}-X_{H_{ \pm}}(u)\right)=0
$$

in compact $C^{\infty}$-topology where $H_{ \pm}$are the Hamiltonians

$$
H_{+}(\tau, t, x)=\kappa^{+}(\tau) H(t, x), \quad H_{-}(\tau, t, x)=\kappa^{-}(\tau) H(t, x) .
$$

We recall (14.1) for the definition of $\kappa^{ \pm}$. We phrase this convergence $u_{j}$ converges to the nodal Floer trajectory $\left(u_{-}, u_{+}\right)$.

In the next section, we will carry out a detailed study of microscopic picture of this convergence near the node $p$.

\section{Controlled nodal degeneration of Floer trajectories}

In this section, we will give a precise description of the degeneration of the solutions

$$
\frac{\partial u}{\partial \tau}+J_{0}\left(\frac{\partial u}{\partial t}-X_{K_{\varepsilon}}(u)\right)=0
$$

to a nodal Floer trajectories as $\varepsilon \rightarrow 0$, where $K_{\varepsilon}$ is the Hamiltonian as defined in (11.3).

We choose a sequence $\varepsilon_{j} \rightarrow 0$ and let $R_{j}$ be any sequence such that $\varepsilon_{j} R_{j} \rightarrow 0$ as $j \rightarrow \infty$, e.g., $R_{j}=-\log \varepsilon_{j} / 2 \pi$. We start with the convergence in the sense of stable maps. 
12.1. Convergence in level 0: stable map convergence. We partition $\mathbb{R} \times S^{1}$ into the union

$$
\mathbb{R} \times S^{1}=\left(-\infty,-R_{j}\right] \cup\left(-R_{j}, R_{j}\right) \cup\left[R_{j}, \infty\right) .
$$

Let $u_{j}$ be a sequence of solutions of (12.1) for $\varepsilon=\varepsilon_{j}$. Then we note that $u_{j}$ satisfies

$$
\begin{aligned}
& \left.\frac{\partial u}{\partial \tau}+J_{0}\left(\frac{\partial u}{\partial t}-\chi\left(\tau-R_{j}+1\right)\right) X_{H}(u)\right)=0 \\
& \text { on }\left(-\infty,-R_{j}+1\right] \times S^{1} \text {, } \\
& \frac{\partial u}{\partial \tau}+J_{0}\left(\frac{\partial u}{\partial t}-\chi\left(-\tau+\left(R_{j}-1\right)\right) X_{H}(u)\right)=0 \\
& \text { on }\left[R_{j}-1, \infty\right) \times S^{1} \text {, and } \\
& \frac{\partial u}{\partial \tau}+J_{0}\left(\frac{\partial u}{\partial t}-\rho_{R_{j}}\left(\tau-R_{j}\right) X_{\varepsilon_{j} f}(u)\right)=0
\end{aligned}
$$

on $\left[-R_{j}, R_{j}\right] \times S^{1}$.

If we consider the translated sequence $u_{j}\left(\tau-\left(R_{j}-1\right), t\right)$, then it satisfies the equation

$$
\frac{\partial u}{\partial \tau}+J_{0}\left(\frac{\partial u}{\partial t}-\chi(\tau) X_{H}(u)\right)=0
$$

and $u_{i}\left(\cdot+\left(R_{j}-1\right), \cdot\right)$ satisfies

$$
\frac{\partial u}{\partial \tau}+J_{0}\left(\frac{\partial u}{\partial t}-\chi(-\tau) X_{H}(u)\right)=0 .
$$

It is important to note that the last two equations do not depend on the parameters $\varepsilon$ (and $R$ ) and so carries the well-defined moduli space of solutions. In the similar vein, we note that as $\varepsilon_{j} \rightarrow 0$, the last equation "converges" to the equation

$$
\frac{\partial u}{\partial \tau}+J_{0} \frac{\partial u}{\partial t}=0
$$

which is again independent of the parameters $\varepsilon_{j}$.

Now we recall our basic hypothesis

$$
0=\mu\left(\left[z_{-}, w_{-}\right] ; H\right)-\mu\left(\left[z_{+}, w_{+}\right] ; H\right)=0 \quad \text { or }-1 .
$$

We will also require all the relevant moduli spaces entering in the gluing constructions are transversal and the almost complex structure $J$ is generic in that all the nodes in this dimension are immersed as proven in Theorem 5.1. This can be always achieved if $(M, \omega)$ is semi-positive. In general, we will apply the machinery of Kuranishi structure [FOn]: Since Theorem 5.1 holds for a generic choice of $J_{0}$ when both $u_{ \pm}$are smooth, and the corresponding smooth moduli space of Floer trajectories are transversal for a generic choice of $J$, we can always put the trivial obstruction bundle on the Floer moduli 
spaces. Nontrivial obstruction bundles will appear only in the sphere bubble components. Therefore, we may safely assume that for a generic choice of $J$, the nodes of all the relevant nodal Floer trajectories are immersed.

We will further assume that $u_{i}$ does not split-off at $\pm \infty$. More precisely, we assume that both $u_{i}\left(\cdot-\left(R_{j}-1\right), \cdot\right)$ and $u_{i}\left(\cdot+\left(R_{j}+1\right), \cdot\right)$ uniformly converge, respectively, as $i \rightarrow \infty$. This will follow from the dimensional restriction by a generic choice of $J$.

Under these hypotheses, a straightforward dimension counting argument, Gromov-Floer compactness and Theorem 11.1 imply

(1) $\left|d u_{j}\right|_{C^{0}}<C$ for all $j$ and $u_{j}$ converges uniformly in fine $C^{\infty}$ topology and

(2) $u_{j}\left(\cdot-\left(R_{j}+1\right), \cdot\right) \rightarrow u_{-}$as $j \rightarrow \infty$ where $u_{-}$satisfies

$$
\frac{\partial u}{\partial \tau}+J_{0}\left(\frac{\partial u}{\partial t}-\chi(\tau) X_{H}(u)\right)=0
$$

and $u_{j}\left(\cdot+\left(R_{j}+K_{j}+1\right), \cdot\right) \rightarrow u_{+}$satisfies

$$
\frac{\partial u}{\partial \tau}+J_{0}\left(\frac{\partial u}{\partial t}-\chi(-\tau) X_{H}(u)\right)=0
$$

We denote by $\operatorname{Glue}\left(u_{-}, u_{+}, u_{0} ; \varepsilon\right)$ the gluing solution constructed in the previous sections out of $u_{-}, u_{+}, u_{0}$ and the parameter $\varepsilon$ with $R=-\log \varepsilon / 2 \pi$. Denote by Glue $(\varepsilon)$ the set of the gluing solutions constructed in Section 10 . In the next section, we will prove that provided $\varepsilon$ is sufficiently small, any solution $u$ of (12.1) "sufficiently $C^{0}$-close to Glue $(\varepsilon)$ " will become indeed Glue $\left(u_{-}, u_{+}, u_{0} ; \varepsilon\right)$ for some choice of $\left(u_{-}, u_{+}\right)$and $u_{0}$. We now make this statement precise in the rest of this section.

We fix conformal identifications

$$
\begin{aligned}
& \varphi_{-}: \Sigma_{-} \rightarrow S^{2} \backslash\{N\}, \quad \varphi_{-}(-\infty)=N, \\
& \varphi_{+}: \Sigma_{+} \rightarrow S^{2} \backslash\{S\}, \quad \varphi_{+}(+\infty)=S,
\end{aligned}
$$

so that they are compatible to the analytic coordinates prescribed near $p_{+} \in$ $\Sigma_{+}$and $q_{-} \in \Sigma_{-}$in Subsection 3.1. As was shown in Subsection 5.2, this will determine the unique points $o_{+} \in \Sigma_{+}, o_{-} \in \Sigma_{-}$, respectively, such that

$$
\varphi_{-}\left(o_{-}\right)=N, \quad \varphi_{+}\left(o_{+}\right)=S .
$$

This will in turn determine a unique conformal identification modulo $\tau$-translations, which we also denote by $\varphi_{ \pm}$

$$
\begin{aligned}
& \varphi_{-}:\left(\Sigma_{-}, q_{-}, o_{-}\right) \rightarrow \mathbb{R} \times S^{1}, \\
& \varphi_{+}:\left(\Sigma_{+}, p_{+}, o_{+}\right) \rightarrow \mathbb{R} \times S^{1} .
\end{aligned}
$$


We then form a disjoint union

$$
\dot{\Sigma}=\dot{\Sigma}_{-} \cup \dot{\Sigma}_{+}
$$

with $o_{-}$and $o_{+}$identified.

We now consider the Floer trajectories $u_{ \pm}: \dot{\Sigma}_{ \pm} \rightarrow M$ with the node $p=u_{-}\left(o_{-}\right)=u_{+}\left(o_{+}\right)$. Since we assume that $u_{-}$and $u_{+}$are immersed at the node $p$ and $J_{0}$-holomorphic, there exists a sufficiently small $\varepsilon_{0}>0$ such that both $u_{-}^{-1}\left(B_{p}^{2 n}\left(\varepsilon_{0}\right)\right)$ and $u_{+}^{-1}\left(B_{p}^{2 n}\left(\varepsilon_{0}\right)\right)$ are conformally isomorphic to $D^{2} \backslash\{0\}$. Denote

$$
\begin{aligned}
& S_{+}=u_{+}^{-1}\left(B_{p}^{2 n}\left(\varepsilon_{0}\right)\right) \subset \dot{\Sigma}_{+}, \\
& S_{-}=u_{-}^{-1}\left(B_{p}^{2 n}\left(\varepsilon_{0}\right)\right) \subset \dot{\Sigma}_{-}
\end{aligned}
$$

and $S=S_{-} \cup S_{+} \subset \dot{\Sigma}_{-} \cup \dot{\Sigma}_{+}=\dot{\Sigma}$.

For further discussion, we will need the following proposition. This is a standard result whose proof can be derived from $[\mathbf{F O n}, \mathbf{M T}]$ and so omitted.

Proposition 12.1. We denote by $\bmod (\Sigma)$ the conformal modulus of the annulus $\Sigma$. Let $(M, \omega, J)$ be an almost Kähler manifold and $\Sigma$ be a Riemann surface of annulus type with $\bmod (\Sigma)=L<\infty$. Suppose that $h: \Sigma \rightarrow M$ is a smooth map satisfying

$$
h(\Sigma) \subset B_{p}^{2 n}(\varepsilon)
$$

and

$$
\bar{\partial}_{J_{R(\varepsilon)}} h+\left(P_{K_{R(\varepsilon), \varepsilon}}\right)_{J_{R(\varepsilon)}}^{(0,1)}(h)=0 .
$$

Identify $\Sigma \cong[-L, L] \times S^{1} \rightarrow M$ conformally. Then there exist $\varepsilon_{0}^{\prime}>0$ and $C, k>0$ depending only on $(M, \omega, J)$ but independent of $h, L$ such that whenever $0 \leq \varepsilon<\varepsilon_{0}^{\prime}$,

$$
|d u|_{g_{J_{0}}}\left(\tau^{\prime}, t^{\prime}\right) \leq C \mathrm{e}^{-\operatorname{dist}\left(\tau^{\prime}, \partial[-L, L]\right)}
$$

for all $\tau^{\prime} \in[-L+1, L-1]$, and

$$
\operatorname{leng}\left(u\left(\tau^{\prime}, \cdot\right)\right) \leq C \mathrm{e}^{-k \operatorname{dist}\left(\tau^{\prime}, \partial[-L, L]\right)} .
$$

We now derive the following lemma from this proposition.

Lemma 12.1. Let $k>0$ be the constant given in Proposition 12.1. There exists $\varepsilon_{0}>0$ such that

$$
u_{j}^{-1}\left(B_{p}^{2 n}\left(\varepsilon_{0}\right)\right)=: \Sigma_{j}\left(\varepsilon_{0}\right)
$$

has a topological type of annulus and decompose $\mathbb{R} \times S^{1}$

$$
\Sigma_{j,-} \cup \Sigma_{j}\left(\varepsilon_{0}\right) \cup \Sigma_{j,+}
$$

such that $\mathbb{R} \times S^{1} \backslash \Sigma_{j}\left(\varepsilon_{0}\right)=\Sigma_{j,-} \coprod \cup \Sigma_{j,+}$. 
Proof. Let $\varepsilon_{0}^{\prime}$ be the constant given in Proposition 12.1. Theorem 11.1 implies that

$$
u_{j}\left(\left[-R_{j}+\frac{1}{3}, R_{j}-\frac{1}{3}\right] \times S^{1}\right) \subset B^{2 n}\left(\varepsilon_{0}^{\prime}\right)
$$

for all sufficiently large $j$ and so the exponential decay (away from the boundary) (12.3) holds. It follows from this that $\Sigma_{j}\left(\varepsilon_{0}^{\prime}\right)$ is of annulus type.

Now we are ready to give the meaning of the stable map convergence of $u_{j}$ to the nodal Floer trajectories $\left(u_{-}, u_{+}\right)$. This is a variation of those given in $[\mathbf{F O n}, \mathbf{H W Z 4}]$ applied to the current circumstance.

Following [HWZ4], we introduce a definition

Definition 12.1 (Definition 4.1, [HWZ4]). A deformation of a compact Riemann surface $(A, j)$ of annulus type is a continuous surjection map $f$ : $A \rightarrow S$ onto the nodal surface, so that $f^{-1}(o)$ is a smooth embedded circle, and

$$
f: A \backslash f^{-1}(o) \rightarrow S \backslash\{o\}
$$

is an orientation preserving diffeomorphism. On $S \backslash\{o\}$ we have the pushed forward complex structure $f_{*} j$.

For each given nodal surface $S$, we recall a construction of a family of deformations in the following way (see $[$ FOn]) parameterized by $\alpha \in \mathbb{C}$ with $|\alpha|$ sufficiently small.

Example 12.1 (Fukaya-Ono, [FOn]). We choose the unique biholomorphic map

$$
\Phi_{\alpha}: T_{o_{-}} S_{-} \backslash\left\{o_{-}\right\} \rightarrow T_{o_{+}} S_{+} \backslash\left\{o_{+}\right\},
$$

such that $u \otimes \Phi_{\alpha}(u)=\alpha$. In terms of analytic coordinates at $o_{-} \in S_{-}$and $o_{+} \in S_{+}$, the coordinate expression of $\Phi_{\alpha}$ is given by the map $\Phi_{\alpha}(z)=\frac{\alpha}{z}$.

We denote $|\alpha|=R_{\alpha}^{-2}$ for $|\alpha|$ sufficiently small and so $R_{\alpha}$ sufficiently large so that the composition

$$
\exp _{S_{-}}^{-1} \circ \Phi_{\alpha} \circ \exp _{S_{+}}: D_{o_{+}}\left(R_{\alpha}^{-\frac{1}{2}}\right) \backslash D_{o_{+}}\left(R_{\alpha}^{-\frac{3}{2}}\right) \rightarrow D_{o_{-}}\left(R_{\alpha}^{-\frac{1}{2}}\right) \backslash D_{o_{-}}\left(R_{\alpha}^{-\frac{3}{2}}\right)
$$

is a diffeomorphism. By composing with the biholomorphism

$$
\begin{aligned}
& {\left[-\ln R_{\alpha}^{-1 / 2}, \ln R_{\alpha}^{1 / 2}\right] \times S^{1} \rightarrow D_{o_{+}}\left(R_{\alpha}^{-\frac{1}{2}}\right) \backslash D_{o_{-}}\left(R_{\alpha}^{-\frac{3}{2}}\right) ;} \\
& \quad(\tau, t) \mapsto \mathrm{e}^{2 \pi((\tau-R)+\mathrm{i} t)}=\mathrm{e}^{-2 \pi R} z
\end{aligned}
$$

with $z=\mathrm{e}^{2 \pi(\tau+\mathrm{i} t)}$ the standard coordinate on $\mathbb{C}$, this diffeomorphism becomes nothing but

$$
\begin{aligned}
{\left[-\ln R_{\alpha}^{-\frac{1}{2}}, \ln R_{\alpha}^{\frac{1}{2}}\right] \times S^{1} } & \rightarrow\left[-\ln R_{\alpha}^{-\frac{1}{2}}, \ln R_{\alpha}^{\frac{1}{2}}\right] \times S^{1} \\
(\tau, t) & \mapsto(-\tau,-t)=\left(\tau^{\prime}, t^{\prime}\right) .
\end{aligned}
$$


We glue the metrics on

$$
D_{o_{+}}\left(R_{\alpha}^{-\frac{1}{2}}\right)-D_{o_{-}}\left(R_{\alpha}^{-\frac{3}{2}}\right)
$$

without changing the metric outside $D_{o_{+}}\left(R_{\alpha}^{-\frac{1}{2}}\right)$ on $\Sigma_{0}$. Identify $D_{o_{+}}\left(R_{\alpha}^{-\frac{1}{2}}\right)$ with an open set in $\mathbb{C} \ni z$ with the standard metric. Consider the biholomorphism $\Phi_{\alpha}: z \rightarrow \frac{\alpha}{z}$, for which we have

$$
\left(\Phi_{\alpha}\right)^{*}|d z|^{2}=\left|\frac{\alpha}{z^{2}}\right|^{2}|d z|^{2} .
$$

Note that on $|z|=R^{-1}$, we have

$$
\begin{gathered}
\Phi_{\alpha}(\{z|| z \mid=\sqrt{\alpha}\})=\{z|| z \mid=\sqrt{\alpha}\}, \\
\left(\Phi_{\alpha}\right)^{*}|d z|^{2}=|d z|^{2} .
\end{gathered}
$$

We choose a function and fix it once and for all

$$
\chi_{R_{\alpha}}:(0, \infty) \rightarrow(0, \infty)
$$

such that

(1) $\left(\Phi_{\alpha}\right)^{*}\left(\chi_{R_{\alpha}}|d z|^{2}\right)=\chi_{R_{\alpha}}|d z|^{2}$,

(2) $\chi_{R_{\alpha}}(r) \equiv 1$, if $r>|\alpha|^{3 / 8}=R_{\alpha}^{-3 / 4}$.

By the definition of $\chi_{R_{\alpha}}$, we can replace the given metric $g_{o_{+}}=|d z|^{2}$ by $\chi_{R_{\alpha}}(|z|)|d z|^{2}$ inside the disc $D^{2}\left(|\alpha|^{1 / 4}\right)$, and denote the resulting metric by $g_{v}^{\prime}$. We would like to emphasize that this modification process is canonical depending only on the fixed complex charts at the singular points and on the choice of $\chi_{R_{\alpha}}$. As a result, this modification process does not add more parameters in the description of deformation of stable curves. Hence, we have constructed a family of stable curves parameterized by a neighborhood of the origin in $T_{o_{+}} S_{+} \otimes T_{o_{-}} S_{-}$. We denote the constructed Riemann surface with the conformal structure constructed in this way by

$$
\left(S_{\alpha}, j_{\alpha}\right)
$$

We set $S_{0}$ to be the given nodal Riemann surface $S$. We can define a surjective continuous map $f_{\alpha}: S_{\alpha} \rightarrow S$ by the projection from the graph of $w=\frac{\alpha}{z}$ to the union of the $z$-axis and $w$-axis that is invariant under the diagonal reflection.

This finishes construction of one-parameter family of deformations of the given nodal Riemann surface. We call this explicit deformation FukayaOno's deformation and will always consider this deformation in the following discussion.

Definition 12.2 (Real deformation). We call the deformation $\left(S_{\alpha}, j_{\alpha} ; f_{\alpha}\right)$ a real deformation if $\alpha \in \mathbb{R}_{+}$. 
We go back to the study of convergence $u_{j}: \mathbb{R} \times S^{1} \cong \dot{\Sigma} \rightarrow M$.

For a given $\mu>0$ and a collection of sufficiently large $R_{\alpha}$, we denote

$$
W_{o, \alpha}(\mu):=\left(D_{o_{+}}(\mu)-D_{o_{+}}\left(R_{\alpha}^{-1}\right)\right) \bigcup\left(D_{o_{-}}(\mu)-D_{o_{-}}\left(R_{\alpha}^{-1}\right)\right)
$$

the prescribed neck region in $S_{\alpha}$. The following definition is essentially the same one as the stable map convergence given in Definition 10.2 [FOn].

Definition 12.3 (Level 0 convergence). We say that $u_{n}$ converges to Glue $\left(u_{-}, u_{+}\right)$in level 0 if

(1) for any $\mu>0,\left.u_{n}\right|_{S_{n} \backslash W_{o}(\mu)} \rightarrow \operatorname{Glue}\left(u_{-}, u_{+}\right)$in $C^{\infty}$ on compact sets,

(2) there exists a sequence of real deformations $f_{n}:\left(S_{n}, j_{n}\right) \rightarrow(S, j)$ such that $\left(f_{n}\right)_{*} j_{n} \rightarrow j_{ \pm}$in compact $C^{\infty}$-topology on $S \backslash\{o\}$,

(3) $\lim _{\mu \rightarrow 0}\left(\lim \sup _{n \rightarrow 0} \operatorname{Diam}\left(u_{n}\left(W_{o, n}(\mu)\right)\right)\right)=0$.

In terms of this definition, the standard definition of stable map convergence of Floer trajectories to a nodal Floer trajectory as given in $[$ FOn,LT1] can be translated into

Proposition 12.2. Consider the partitions $\left(-L_{j}, L_{j}\right)$ associated to the surface $\Sigma_{0, j}=u_{j}^{-1}\left(B\left(\varepsilon_{0}, p\right)\right)$. Then the maps $u_{j}: \Sigma_{0, j} \rightarrow M$ converge to the nodal Floer trajectory

$$
\left.\operatorname{Glue}\left(u_{-}, u_{+}\right)\right|_{S\left(\varepsilon_{0}\right)},
$$

where $S\left(\varepsilon_{0}\right)=S_{-}\left(\varepsilon_{0}\right) \cup S_{+}\left(\varepsilon_{0}\right)$.

Note that the level 0 convergence does not reflect the immersion property of the nodes. It turns out that the level 0 convergence to nodal trajectories with immersed nodes has a finer convergence property, which we now explain.

12.2. One-jet convergence to nodal curves with immersed nodes. Now we are ready to give the precise meaning of the convergence $u_{j}$ to $\left(u_{-}, u_{+}, u_{0}\right)$, where $u_{0}$ is a local model obtained in Section 6 .

We start with the description of the sequence of Floer trajectories $u_{j}$ over the central region $\Sigma_{j}\left(\varepsilon_{0}\right)$. Fix a sufficiently small $\varepsilon_{0}>0$, for which Proposition 12.1 holds. We choose a conformal diffeomorphism

$$
\psi_{j, \text { int }}:\left[-L_{j}, L_{j}\right] \rightarrow \Sigma_{j}\left(\varepsilon_{0}\right)
$$

with $2 L_{j}=\bmod \left(\Sigma_{j}\left(\varepsilon_{0}\right)\right)$. We denote the corresponding conformal coordinates by $\left(\tau^{\prime}, t^{\prime}\right)$. We would like to emphasize that this coordinates $\left(\tau^{\prime}, t^{\prime}\right)$ may not be the same one as the original coordinates $(\tau, t)$ in $\mathbb{R} \times S^{1}$.

Applying Proposition 12.1 to the maps

$$
h_{j}=u_{j} \circ \psi_{j, \text { int }},
$$

we obtain 
Corollary 12.1. There exist $\varepsilon_{0}>0$, a sequence $\varepsilon_{i}^{\prime} \rightarrow 0$ and a subsequence $j_{i}$ of $j$ 's in turn so that

(1) $u_{i}$ is embedded on $u_{i}^{-1}\left(B_{p}^{2 n}\left(\varepsilon_{0}\right) \backslash B_{p}^{2 n}\left(\varepsilon_{i}^{\prime}\right)\right)$ and $u_{i}^{-1}\left(B_{p}^{2 n}\left(\varepsilon_{0}\right) \backslash B_{p}^{2 n}\left(\varepsilon_{i}^{\prime}\right)\right)$ is a disjoint union of two components $\Sigma_{i, \varepsilon_{i}^{\prime} \leq r \leq \varepsilon_{0}}^{ \pm}$of cylindrical type.

$$
\varepsilon_{i}^{\prime} / \varepsilon_{j_{i}} \rightarrow \infty, \quad \bmod \left(\Sigma_{i, \varepsilon_{i}^{\prime} \leq r \leq \varepsilon_{0}}^{ \pm}\right) \rightarrow \infty
$$

Proof. The first statement is an immediate consequence of a diagonal sequence argument from the stable map convergence $u_{i}$ and the immersion property of $u_{ \pm}$.

For the second statement, we pick any sequence $\varepsilon_{i}^{\prime} \rightarrow 0$ and consider the $\operatorname{modulus} \bmod \left(u_{j}^{-1}\left(B_{p}^{2 n}\left(\varepsilon_{i}^{\prime}\right)\right)\right.$. By the first statement, we have

$$
\lim _{j \rightarrow \infty} \bmod \left(u_{j}^{-1}\left(B_{p}^{2 n}\left(\varepsilon_{i}^{\prime}\right)\right)=\infty\right.
$$

for each fixed $i$. Take the subsequence $j_{i}$ of $j$ so that

$$
\varepsilon_{i}^{\prime} / \varepsilon_{j_{i}}, \quad \bmod \left(u_{j}^{-1}\left(B_{p}^{2 n}\left(\varepsilon_{i}^{\prime}\right)\right)\right) \geq i
$$

for each $i$ : this is possible since $\varepsilon_{j} \rightarrow 0$. This finishes the proof.

By renumbering $j_{i}$, we will just denote $j_{i}$ by $i$ and so we are given two sequences

$$
\varepsilon_{i}, \varepsilon_{i}^{\prime} \rightarrow 0, \quad \varepsilon_{i}^{\prime} / \varepsilon_{i} \rightarrow \infty
$$

and $\bmod \left(u_{i}^{-1}\left(B_{p}^{2 n}\left(\varepsilon_{i}^{\prime}\right)\right)=: 2 L_{i}^{\prime} \rightarrow \infty\right.$ as $i \rightarrow \infty$. We will assume this for the rest of this section.

Now we define a rescaled map

$$
\widetilde{u_{i, \text { int }}}:\left[-L_{i}^{\prime}, L_{i}^{\prime}\right] \times S^{1} \rightarrow \mathbb{C}^{n}
$$

by

$$
\widetilde{u_{i, \text { int }}}(z)=\frac{1}{\epsilon_{i}}\left(u_{i} \circ \psi_{i, \text { int }}\right)(z)
$$

and study its convergence behavior.

We consider the decomposition of the Riemann surface

$$
\mathbb{R} \times S^{1} \cong \dot{\Sigma}=\Sigma_{i,-} \cup \Sigma_{i, 0} \cup \Sigma_{i,+},
$$

where $\Sigma_{i, 0}=u_{i}^{-1}\left(B^{2 n}\left(\varepsilon_{i}^{\prime}\right)\right)$ and

$$
\mathbb{R} \times S^{1} \backslash \Sigma_{j, 0}=\Sigma_{j,-} \cup \Sigma_{j,+} .
$$

We denote the translated sequences

$$
\begin{aligned}
& u_{i,-}^{\prime}=u_{i}\left(\cdot-\left(R_{i}+1\right), \cdot\right):\left(-\infty, R_{i}\right] \times S^{1} \rightarrow M, \\
& u_{i,+}^{\prime}=u_{i}\left(\cdot+\left(R_{i}+1\right), \cdot\right):\left[-R_{i}, \infty\right) \times S^{1} \rightarrow M
\end{aligned}
$$


and their conformal reparameterizations by

$$
\begin{aligned}
& v_{i,-}=u_{i}^{\prime} \circ \varphi_{-}: \varphi_{-}^{-1}\left(\left(-\infty, R_{i}\right] \times S^{1}\right) \rightarrow M, \\
& v_{i,+}=u_{i}^{\prime} \circ \varphi_{+}: \varphi_{+}^{-1}\left(\left[-R_{i}, \infty\right) \times S^{1}\right) \rightarrow M .
\end{aligned}
$$

It is easy to see from the definitions that we can choose $R_{i}=R_{i}\left(\varepsilon_{i}\right)$ so that $\varphi_{-}^{-1}\left(\left(-\infty, R_{i}\right] \times S^{1}\right) \supset S^{2} \backslash D_{S}^{2}\left(C \varepsilon_{i}\right), \quad \varphi_{-}^{-1}\left(\left[-R_{i}, \infty\right) \times S^{1}\right) \supset S^{2} \backslash D_{N}^{2}\left(C \varepsilon_{i}\right)$ for some constant $C>0$ independent of $i$.

Now we are ready to give the main definition of the refined convergence. As before $u_{0}$ stands for a local model obtained in Section 6.

Definition 12.4 ( $\left\{\varepsilon_{i}\right\}$-controlled one-jet convergence). We say that a sequence $u_{\varepsilon_{i}}$ of solutions for (12.1) converges to $\left(u_{-}, u_{+} ; u_{0}\right)$ in the $\left\{\varepsilon_{i}\right\}$ controlled way if the following holds :

(1) $u_{\varepsilon_{i}}$ converges to Glue $\left(u_{-}, u_{+}\right)$in level 0 ,

(2) we have $\bmod \left(\Sigma_{i, 0}^{\prime}\right)=2 L_{i}^{\prime} \rightarrow \infty$,

(3) there exists a sequence of automorphisms $g_{v, \lambda}$ given by $g_{v, \lambda}(u)=$ $\lambda u+v$ for some vectors $v_{i} \in \mathbb{C}^{n}$ and $\lambda_{i} \in \mathbb{R}$ such that we have the inequality

$$
\left|\nabla^{k}\left(g_{v_{i}, \lambda_{i}}^{-1}\left(\frac{1}{\varepsilon_{i}} u_{i} \circ \psi_{i, \text { int }}+\tau \vec{a}\right)-u_{0}\right)(\tau, t)\right| \leq \min \left(\delta_{k, i}, C_{k} \mathrm{e}^{-c_{k}\left|\tau-L_{i}^{\prime}\right|}\right)
$$

on $\left[-L_{i}^{\prime}, L_{i}^{\prime}\right] \times S^{1}$ in the given Darboux chart at $p$ with respect to the cylindrical metrics on $\mathbb{R} \times S^{1}$ and $g_{\mathbb{C}^{n}}^{\prime}$.

Surjectivity proof will be finished by the following convergence theorem.

Theorem 12.1. Suppose that $u_{-}, u_{+}$are immersed at the node

$$
p=u_{-}\left(o_{-}\right)=u_{+}\left(o_{+}\right) .
$$

Let Glue $\left(u_{-}, u_{+}\right)$be the nodal Floer trajectory formed by $u_{-}$and $u_{+}$ with nodal points $p=u_{-}(o-)=u_{+}\left(o_{+}\right)$. Suppose that $u_{n}$ converges to Glue $\left(u_{-}, u_{+}\right)$in level 0 . Then there exists a subsequence $u_{n_{i}}$ and a sequence $\varepsilon_{i} \rightarrow 0$ such that $u_{n_{i}}$ converges to $\left(u_{-}, u_{+} ; u_{0}\right)$ in the $\left\{\varepsilon_{i}\right\}$-controlled way.

We will give the proof of this theorem in the next section.

Once we prove this theorem, the well-known argument by Donaldson $[\mathbf{D}]$ proves the following which will finish the proof of surjectivity. We omit the details of this last step but refer to Section 62.7 of Chapter 10 of [FOOO2] for relevant details of this last step in a similar context.

Theorem 12.2. Let $R(\varepsilon)=-\frac{1}{2 \pi} \log \varepsilon$ and $K_{\varepsilon}$ be the Hamiltonian as defined in (11.3). There exists small constants $\varepsilon_{1}, \varepsilon_{2}$ with $\varepsilon_{1}<\varepsilon_{2}^{100}$ such that for any $0<\varepsilon<\varepsilon_{1}$ and any solution $u: \mathbb{R} \times S^{1} \rightarrow M$ of

$$
\bar{\partial}_{J_{\varepsilon}} u+P_{K_{\varepsilon}, J_{\varepsilon}}^{(1,0)}(u)=0
$$


satisfying

$$
\max _{z \in \mathbb{R} \times S^{1}} \operatorname{dist}(u, \operatorname{Glue}(\varepsilon))<\varepsilon_{2}
$$

indeed has the form $u=\operatorname{Glue}\left(u_{-}, u_{+}, u_{0} ; \varepsilon\right)$ for some $u_{-}, u_{+}$and $u_{0}$.

Here the choice of exponent "100" is not significant which is made imitating the statement of Theorem 62.2 [FOOO2].

The following proposition will be important in the energy estimates needed to prove the above theorem. Again this is the analog to Proposition 62.79 [FOOO2] in the current context. Here since we consider the case where $\gamma_{+, j}$ converges to $\gamma_{a^{+}}$in $C^{0}$-topology, we can write

$$
\gamma_{+, j}(t)-\gamma_{a^{+}}(t):=\exp _{\gamma_{+}(t)}^{-1}\left(\gamma_{+, j}(t)\right)
$$

for the unit vector

$$
a^{+}:=\lim _{\tau \rightarrow \infty} \frac{d u^{+}\left(\frac{\partial}{\partial \tau}\right)}{\left|d u^{+}\left(\frac{\partial}{\partial \tau}\right)\right|} .
$$

Similar remark applies to $\gamma_{-, j}$.

We identify a Darboux neighborhood of $p$ with an open neighborhood of $0 \in \mathbb{C}^{n} \cong T_{p} M$.

Proposition 12.3. For each given $k$, there exist $I_{0}, R_{0}$ and constant $o\left(i, R_{0} \mid k\right)$ with

$$
\lim _{i \rightarrow \infty} \lim _{R_{0} \rightarrow \infty} o\left(i, R_{0} \mid k\right)=\infty
$$

such that for all $-\frac{1}{2 \pi} \log \varepsilon_{i}^{\prime}+R_{0} \leq s \leq-\frac{1}{2 \pi} \log \varepsilon_{0}-R_{0}$ the followings hold:

(1) $s$ is a regular value of $s \circ u_{i}$ and the curve $u_{i}\left(\Sigma_{i}\left(\varepsilon_{0}\right)\right) \cap\left(\{s\} \times S^{2 n-1}\right)$ is parameterized by the union of two disjoint circles $\gamma_{i, s}^{ \pm}: S^{1} \times S^{2 n-1}$ for which we have

$$
\left|\nabla^{k}\left(\gamma_{i, s}^{ \pm}-\gamma_{a^{ \pm}}\right)\right| \leq o\left(i, R_{0} \mid k\right) .
$$

(2) For $s_{1} \in\left[-\frac{1}{2 \pi} \log \varepsilon_{i}^{\prime}+R_{0},-\frac{1}{2 \pi} \log \varepsilon_{0}-R_{0}\right]$, the set

$$
\Sigma_{i, s_{1}-1 \leq s \leq s_{1}+1}=u_{i}\left(\Sigma_{i}\right) \cap\left(\left[s_{1}-1, s_{1}+1\right] \times S^{2 n-1}\right)
$$

is a disjoint union of two components $\Sigma_{i, s_{1}-1 \leq s \leq s_{1}+1}^{ \pm}$such that each of $\Sigma_{i, s_{1}-1 \leq s \leq s_{1}+1}^{ \pm}$has a parameterization

$$
u_{i, s_{1}-1 \leq s \leq s_{1}+1}^{ \pm}:[-1 / 2 \pi, 1 / 2 \pi] \times S^{1} \rightarrow \Sigma_{i, s_{1}-1 \leq s \leq s_{1}+1}^{ \pm}
$$

for which we have

$$
\left|\nabla^{k}\left(u_{i, s_{1}-1 \leq s \leq s_{1}+1}^{ \pm}-u_{a^{ \pm}, s_{1}}^{\text {flat }}\right)\right|<o\left(i, R_{0} \mid k\right),
$$

where

$$
u_{a^{ \pm}, s_{1}}^{\text {flat }}(\tau, t)=\left(2 \pi \tau+s_{1}, \gamma_{a^{ \pm}}(t)\right)
$$


Proof. We first note that regularity of $s$ follows from Corollary 12.1.

Consider the composition

$$
v_{-}:=u_{-} \circ \varphi_{-}^{-1}: S^{2} \backslash\{S\} \rightarrow M .
$$

This map $v_{-}$extends smoothly to $S^{2}$ and its derivative $d v_{-}(S) \neq 0$ by the immersion assumption on the node. Now we consider the translated sequence $u_{j}^{\prime}:=u_{j}\left(\cdot-\left(R_{j}+1\right), \cdot\right)$ which converges to $u_{-}$in compact $C^{\infty}$ topology on $\left(-\infty, R_{j}\right] \times S^{1}$ as $j \rightarrow \infty$ and define

$$
v_{j}:=u_{j}^{\prime} \circ \varphi_{-}^{-1}: \varphi_{-}\left(\left(-\infty, R_{j}\right] \times S^{1}\right) \rightarrow M
$$

as before. Then by the hypothesis of level zero convergence, $S^{2} \backslash \varphi_{-}((-\infty$, $\left.\left.R_{j}+1\right] \times S^{1}\right)$ shrinks to the point $\{S\}$ as $j \rightarrow \infty$ and $v_{j} \rightarrow v_{-}$in compact $C^{\infty}$ topology and $v_{-}$is immersed at $S$.

For the rest of the statements, we will prove them by contradiction. Suppose to the contrary. Then we can choose a sequence $s_{i}$ with

$$
s_{i}-\frac{1}{2 \pi} \log \varepsilon_{i}^{\prime}, \quad \frac{1}{2 \pi} \log \varepsilon_{0}-s_{i} \rightarrow \infty
$$

such that one of the following alternatives must hold:

(1) There exist $k$ and $c>0$ such that

$$
\left|\nabla^{k}\left(\gamma_{a^{+}}-\gamma_{i, s_{i}}^{+}\right)\right|>c
$$

or

$$
\left|\nabla^{k}\left(\gamma_{a^{-}}-\gamma_{i, s_{i}}^{-}\right)\right|>c
$$

for any parameterization $\gamma_{i, s_{i}}^{ \pm}$of $u_{i}\left(\Sigma_{i}^{ \pm}\right) \cap\left(\left\{s_{i}\right\} \times S^{2 n-1}\right)$.

(2) There exist $k$ and $c>0$ such that

$$
\left|\nabla^{k}\left(u_{i, s_{i}-1 \leq s \leq s_{i}+1}-u_{a^{ \pm}, s_{i}}^{\text {flat }}\right)\right|>c
$$

for any parameterization $u_{i, s_{i}-1 \leq s \leq s_{i}+1}^{ \pm}$of $\Sigma_{i, s_{1}-1 \leq s \leq s_{1}+1}^{ \pm}$.

In terms of $r$ coordinates, we have

$$
\begin{aligned}
{\left[s_{i}-1, s_{i}+1\right] \times S^{2 n-1} } & \leftrightarrow\left[\mathrm{e}^{s_{i}-1}, \mathrm{e}^{s_{i}+1}\right] \times S^{2 n-1} \\
& =\left[\varepsilon_{0}^{1 / 2 \pi} \mathrm{e}^{-K_{i}-1}, \varepsilon_{0}^{1 / 2 \pi} \mathrm{e}^{-K_{i}+1}\right] \times S^{2 n-1}
\end{aligned}
$$

for $K_{i}:=\frac{1}{2 \pi} \log \varepsilon_{0}-s_{i} \rightarrow \infty$. Since $v_{-}$is immersed at $\{S\}$ and $v_{-}(S)=p$, the subset

$$
v_{-}\left(\left[\varepsilon_{0}^{1 / 2 \pi} \mathrm{e}^{-K_{j}-1}, \varepsilon_{0}^{1 / 2 \pi} \mathrm{e}^{-K_{i}+1}\right] \times S^{2 n-1}\right):=A_{i} \subset S^{2} \backslash\{S\}
$$

is of annulus type and shrinks to the point $S$ as $j \rightarrow \infty$. Therefore, by taking a diagonal sequence argument and using the immersion property of $v_{-}$at $S$, if necessary, we may assume

$$
\left|\nabla^{k}\left(v_{i}-v_{-}\right)\right|_{A_{i}} \rightarrow 0 .
$$


This is then translated into

$$
\left|\nabla^{k}\left(u_{i, s_{i}-1 \leq s \leq s_{i}+1}-u_{a^{ \pm}, s_{i}}^{\text {flat }}\right)\right| \rightarrow 0
$$

as $i \rightarrow \infty$. This in particular rules out the second possibility.

On the other hand, the immersion property of $u_{-}$at $o_{-}$implies that

$$
\left|u_{+}(z)-z \cdot a^{+}\right|=O\left(|z|^{2}\right) .
$$

Therefore, we have

$$
|| u_{+}(z)|-| z|| a^{-}||=\left|r\left(u_{+}(z)\right)-r\left(z \cdot a^{+}\right)\right| \leq O\left(|z|^{2}\right) .
$$

Since $\Theta\left(u_{+}(z)\right)=\frac{u_{+}(z)}{\left|u_{+}(z)\right|}$, we obtain

$$
\begin{aligned}
\left|\Theta\left(u_{+}(z)\right)-\Theta\left(z \cdot a^{+}\right)\right| & =\left|\frac{u_{+}(z)}{\left|u_{+}(z)\right|}-\frac{z \cdot a^{+}}{|z|\left|a^{-}\right|}\right| \\
& \leq \frac{\left|u_{+}(z)-z \cdot a^{+}\right|}{|z|\left|a^{-}\right|}+\frac{\| u_{+}(z)|-| z|| a^{-}||}{|z|\left|a^{-}\right|} \leq O(|z|) .
\end{aligned}
$$

Similarly, we have

$$
\begin{aligned}
\left|\gamma_{j, s_{i}}^{+}-\gamma_{a^{+}}\right| & =\left|\Theta\left(u_{j,+}(z)\right)-\Theta\left(z \cdot a^{+}\right)\right| \\
& =\left|\Theta\left(u_{+}(z)\right)-\Theta\left(z \cdot a^{+}\right)\right|+\left|\Theta\left(u_{j,+}(z)\right)-\Theta\left(z \cdot a^{+}\right)\right| .
\end{aligned}
$$

For the first term, we have

$$
\left|\Theta\left(u_{+}(z)\right)-\Theta\left(z \cdot a^{+}\right)\right| \leq O(|z|)
$$

and for the second term, we have

$$
\lim _{i \rightarrow \infty}\left|\Theta\left(u_{i,+}(z)\right)-\Theta\left(z \cdot a^{+}\right)\right|=0 .
$$

Therefore, we have obtained

$$
\left|\Theta\left(u_{+}(z)\right)-\Theta\left(u_{i,+}(z)\right)\right| \rightarrow 0
$$

since $z$ satisfies $\varepsilon_{0}^{1 / 2 \pi} \mathrm{e}^{-K_{i}-1} \leq|z| \leq \varepsilon_{0}^{1 / 2 \pi} \mathrm{e}^{-K_{i}+1}$ and $K_{i} \rightarrow \infty$ as $j \rightarrow \infty$.

Combining (12.9) and (12.10), we can prove

$$
\left|\nabla^{k}\left(\gamma_{a^{-}}-\gamma_{i, s_{i}}^{-}\right)\right| \rightarrow 0
$$

inductively over $k=0, \ldots$, as $\gamma_{i, s_{i}}^{-}(t)=\Theta\left(u_{i,+}^{-1}\left(B_{p}\left(\mathrm{e}^{s_{i}}\right)\right)\right)$. This contradicts to the hypothesis and so the proposition is proved. 


\section{Surjectivity of the scale-dependent gluing family}

The main goal of this section is to prove Theorem 12.1, which will imply that the enhanced resolutions of Floer nodal trajectories $\left(u_{-}, u_{+}, u_{0}\right)$ exhaust all the solutions of (11.4), which are close to those of the enhanced nodal trajectories $\left(u_{-}, u_{+}\right)$in a suitable sense.

To prepare the proof, we consider the map

$$
\widetilde{u}_{i}: \Sigma_{i}\left(\varepsilon_{0}\right) \rightarrow T_{p} M
$$

defined by

$$
\widetilde{u}_{i}(\tau, t)=\frac{1}{\varepsilon_{i}}\left(\exp _{p}^{I}\right)^{-1} \circ u_{i}
$$

where $\Sigma_{i}\left(\varepsilon_{0}\right)=u_{i}^{-1}\left(B_{p}^{2 n}\left(\varepsilon_{0}\right)\right)$.

We denote the pull-back almost complex structure on $\left(\exp _{p}^{I}\right)^{-1}\left(B_{p}^{2 n}\left(\varepsilon_{0}\right)\right)$ $\subset T_{p} M$ by $\widetilde{J}_{i}$ which is defined by

$$
\widetilde{J}_{i}=\left(\exp _{p}^{I} \circ R_{\varepsilon_{i}}\right)^{*} J_{0}
$$

on $B_{p}^{2 n}\left(\varepsilon_{0} / \varepsilon_{i}\right) \subset T_{p} M$. Then $\widetilde{u}_{i}$ satisfies the equation

$$
\bar{\partial}_{\widetilde{J}_{i}} \widetilde{u}_{i}+\left(P_{K_{R_{i}, \varepsilon_{i}}^{(1,0)}}\right) \widetilde{J}_{i}\left(\widetilde{u}_{i}\right)=0 .
$$

We first describe the metrics on the domain $\mathbb{C}$ and the target $\mathbb{C}^{n}$, with which we evaluate the $C^{k}$ norms of $\xi_{i}$ 's.

For $\varepsilon_{i}^{\prime}$ chosen before it follows, by choosing $\varepsilon_{0}$ smaller if necessary, that $\Sigma_{i}\left(\varepsilon_{0}\right) \backslash \Sigma_{i}\left(\varepsilon_{i}^{\prime}\right)$ is a disjoint union of two domains of cylindrical type. We denote

$$
\widetilde{u}_{i}^{-1}\left(B_{p}^{2 n}\left(\varepsilon_{0}\right)\right) \backslash \widetilde{u}_{i}^{-1}\left(B_{p}^{2 n}\left(\varepsilon_{i}^{\prime}\right)\right)=C_{i, 1}\left(\varepsilon_{i}^{\prime}, \varepsilon_{0}\right) \cup C_{i,+}\left(\varepsilon_{i}^{\prime}, \varepsilon_{0}\right) .
$$

Whenever there is no danger of ambiguity, we will just denote $C_{i, \pm}$ for $C_{i, \pm}\left(\varepsilon_{i}^{\prime}, \varepsilon_{0}\right)$, respectively.

We recall that we have used the metrics as follows: for the target, we use the metric, denoted by $g_{\mathbb{C}^{n}}^{\prime}$, to satisfy the following properties:

(1) $g_{\mathbb{C}^{n}}^{\prime}$ is a flat Euclidean metric on the Euclidean ball $B^{2 n}(2)$ of radius 2.

(2) Outside the (Euclidean) ball $B^{2 n}(4)$, it is the standard product metric on $[\log 4, \infty) \times S^{2 n-1}(3)$. (Here $S^{2 n-1}(3)$ is the round sphere of radius 3 .

(3) $g_{\mathbb{C}^{n}}$ is of nonnegative curvature.

For the domain, we require the metric, denoted by $g_{\mathbb{C}}^{\prime}$, to have totally geodesic boundary and to satisfy the following properties:

(1) $g_{\mathbb{C}}^{\prime}$ is a flat Euclidean metric on the Euclidean ball $B^{2}(1)$ of radius 1 .

(2) Outside the (Euclidean) ball $B^{2}(2)$ of radius $2, g_{\mathbb{C}}^{\prime}$ is the standard product metric $[0, \infty) \times[0,3 \pi / 2]$.

(3) $g_{\mathbb{C}}^{\prime}$ is of nonnegative curvature. 
We now recall that for any contact hypersurface $(N, \xi)$ of a symplectic manifold $(M, \omega)$ has the canonical co-orientation. If a smooth map $u: \Sigma \rightarrow$ $M$ from an oriented surface $\Sigma$ is transversal to a contact hypersurface $N \subset$ $M$, then the preimage $u^{-1}(N)$ has a natural orientation induced by the coorientation of $N \subset M$. Call this the induced orientation on $u^{-1}(N)$ and denote $o_{\text {ind }}$.

When $\Sigma$ is given a complex structure $j$, it carries the complex orientation on it and its boundary $\partial \Sigma$ has the boundary orientation $o_{\text {bdy }}$ defined by the convention

$$
\vec{n} \oplus o_{\mathrm{bdy}}=o_{\Sigma},
$$

where $\vec{n}$ is the unit normal outward to $\Sigma$ on the boundary.

Now assume that $\Sigma$ is oriented and $\partial \Sigma=\coprod_{j} \partial_{j} \Sigma$, where each $\partial_{i} \Sigma$ denotes a connected component of $\partial \Sigma$. If $u: \Sigma \rightarrow M$ is transversal to a contact hypersurfaces $N_{j} \subset M$ and $u^{-1}\left(N_{j}\right)=\partial_{j} \Sigma$, then $\partial_{j} \Sigma$ carries two orientations $o_{\text {ind }}$ and $o_{\text {bdy }}$.

Definition 13.1. Let $(u, \Sigma)$ as above. We say that a component $\partial_{i} \Sigma$ is an outside boundary if $o_{\text {ind }}=o_{\mathrm{bdy}}$, and an inside boundary if $o_{\text {ind }}=-o_{\mathrm{bdy}}$. We denote by $\partial_{\text {out }} \Sigma$ the union of outside boundaries and by $\partial_{\text {in }} \Sigma$ the union of inside boundaries.

Theorem 13.1. Let $\widetilde{u}_{i}$ satisfy (13.1). There exist $a_{i}$ and $\delta_{k, i}>0$, such that $\lim _{i \rightarrow \infty} a_{i, \pm}=a_{ \pm}, \lim _{i \rightarrow \infty} \delta_{k, i}=0$ and $u_{i}$ satisfies the following properties:

(1) There exists an open subset $\mathcal{U}_{i, \text { out }}$ of $\Sigma_{i}\left(\varepsilon_{0}\right)$ containing $C_{i,+} \cup C_{i,-}$, a sequence $L_{i, \pm} \rightarrow \infty$ such that there exists a biholomorphic embedding

$$
\begin{aligned}
& \psi_{i, \text { neck, },}:\left[0,2 L_{i,+}\right] \times S^{1} \rightarrow C_{i,+} \\
& \psi_{i, \text { neck,- }}:\left[-2 L_{i,-}, 0\right] \times S^{1} \rightarrow C_{i,-}
\end{aligned}
$$

such that $\psi_{i, \text { neck, } \pm}$ satisfies

$$
\left.\mid \nabla^{k}\left(\left(\widetilde{u}_{i} \circ \psi_{i, \text { neck, }}\right)-u_{a_{i}}^{\text {flat }}\right)\right) \mid(\tau, t)<C_{k} \mathrm{e}^{-c_{k} \min \left\{|\tau|,\left|L_{i, \pm} \pm \tau\right|\right\}} .
$$

on $\left[0,2 L_{i,+}\right] \times S^{1}$ (or on $\left[-2 L_{i,-}, 0\right] \times S^{1}$, respectively). Here $c_{k}, C_{k}$ are independent of $i$ and we put

$$
u_{a_{i, \pm}}^{f l a t}(\tau, t)=\left(2 \pi \tau, \gamma_{a_{i, \pm}}(t)\right)
$$

and use the cylindrical metrics for both the domain and the target.

(2) There exist a sequence $L_{i, 0} \rightarrow \infty$, open sets $\mathcal{U}_{i \text {,int }} \subset \Sigma_{i}\left(\varepsilon_{0}\right)$ and a biholomorphic map

$$
\psi_{i, i n t}:\left[-L_{i, 0}, L_{i, 0}\right] \times S^{1} \rightarrow \mathcal{U}_{i, i n t}
$$

with the following properties:

(a) $\mathcal{U}_{i, \text { int }} \cap \mathcal{U}_{i, \text { out }}=\operatorname{Im}\left(\psi_{i, \text { int }}\right) \cap \operatorname{Im}\left(\psi_{i, \text { neck }}\right)$. 
(b) $u_{i} \circ \psi_{i, \text { int }}$ satisfies Definition 12.4, i.e., there exists a sequence of automorphisms $g_{v_{i}, \lambda_{i}}$ of $\mathbb{C}^{n}$ such that

$$
\begin{gathered}
\left.\mid \nabla^{k} g_{v_{i}, \lambda_{i}}^{-1}\left(\frac{1}{\varepsilon_{i}} u_{i} \circ \psi_{i, i n t}(\tau, t)+\tau \vec{a}\right)\right)-u_{0}(\tau, t) \mid \\
\leq \min \left(\delta_{k, i}, C_{k} \mathrm{e}^{-c_{k} \min \left\{\left|\tau \pm L_{i, 0}\right|\right\}}\right) \\
\text { on }\left[-L_{i, 0}, L_{i, 0}\right] \times S^{1} \text { with in the cylindrical metrics on } \mathbb{R} \times S^{1} \text { and }
\end{gathered}
$$$$
g_{\mathbb{C}^{n}}^{\prime} .
$$

Here we use conformal parameterizations on $C_{i,+}$ and $C_{i,-}$ given as above because the boundary orientation $o_{\text {bdy }}$ on $C_{i,+} \cap \partial B_{p}\left(\varepsilon_{i, \text { out }}\right)$ of the complex orientation on $C_{i,+}$ coincide with the above induced orientation $o_{\text {ind }}$, while that of $C_{i,-}$ is opposite.

The remaining section will be occupied by the proof of this theorem.

We start with the following characterization of small energy cylinders, which can be proved by the same method as in $[$ Ho, HWZ4, FOOO2]. We denote by $u_{a, s_{1}}^{\text {flat }}$ the cylindrical strip defined by

$$
u_{a, s_{1}}^{\text {flat }}(\tau, t)=\left(s_{1}+\tau, \gamma_{a}(t)\right)
$$

as before.

Theorem 13.2 (Theorem 1.3 [HWZ4], Theorem 62.85 [FOOO2]). Let $R>0$ be given and let $u:[-R, R] \times S^{1} \rightarrow \mathbb{R} \times S^{2 n-1}$ be a $J_{0}$-holomorphic map. For each $E_{0}>0$ and $k$ there exist positive constants $e_{0}, R_{0}, c_{k}$ and $C_{k}$ as follows: Whenever $u$ satisfies

(1) $E(u) \leq E_{0}$,

(2) $R \geq R_{0}$,

(3) $E_{d \lambda}(u) \leq e_{0}$,

(4) the loop $u_{0}(t):=u(0, t)$ satisfies

$$
\int u_{0}^{*} \lambda \leq 3 \pi
$$

we can find $a \in S^{2 n-1}$ and $s_{1} \in \mathbb{R}$ for which we have

$$
\left|\nabla^{k}\left(u-u_{a, s_{1}}^{\text {flat }}\right)\right|(\tau, t) \leq C_{k} \mathrm{e}^{-c_{k}(R-|\tau|)}
$$

on $(\tau, t) \in[-R+10, R-10] \times[0,1]$.

13.1. Convergence in the neck regions. We define

$$
\mathcal{U}_{i, \text { out }}=\Sigma_{i}\left(\varepsilon_{0}\right) \backslash u_{i}^{-1}\left(B_{p}^{2 n}\left(\varepsilon_{i, \text { in }}\right)\right) .
$$

In this subsection, we will study convergence of $\widetilde{u}_{i}$ on the neck regions

$$
u_{i}^{-1}\left(B_{p}^{2 n}\left(\varepsilon_{i, \text { out }}\right)\right) \backslash u_{i}^{-1}\left(B_{p}^{2 n}\left(\varepsilon_{i, \text { in }}\right)\right)
$$

for a choice of two sequences $\varepsilon_{i \text {,out }}>\varepsilon_{i \text {,in }} \gg \varepsilon_{i}$ such that

$$
\varepsilon_{i, \text { out }}, \varepsilon_{i, \text { in }} \rightarrow 0, \quad\left|\log \varepsilon_{i, \text { out }}-\log \varepsilon_{i, \text { in }}\right| \rightarrow \infty
$$


as $i \rightarrow \infty$. It follows from Proposition 12.3 that for a suitable choice of $\varepsilon_{i \text {,out }}$, $u_{i}^{-1}\left(B_{p}^{2 n}\left(\varepsilon_{i, \text { out }}\right)\right)$ define a sequence of open Riemann surfaces which converges to a conformal cylinder $\mathbb{R} \times S^{1}$. Furthermore the standard symplectic area of the rescaled maps $\widetilde{u}_{i}$ converges to infinity, but its end behavior is controlled by the hypotheses that $u_{i}$ converges to the nodal curve $\left(u_{-}, u_{+}\right)$whose node is immersed.

While the standard argument for the closed Riemann surface does not apply to the sequence of the rescaled maps $\widetilde{u}_{i}$ defined on open Riemann surfaces $\Sigma_{i}^{\prime}=u_{i}^{-1}\left(B_{p}^{2 n}\left(\varepsilon_{i, \text { out }}\right)\right)$, the imposed end behavior enables us to apply the strategy employed by Hofer $[\mathbf{H o}]$ estimating the horizontal and vertical energies separately. However in our current circumstance, we need to apply Hofer's strategy to the case where the target manifold is neither complete nor cylindrical but only approximately cylindrical.

We need to concern parameterization of maps $\widetilde{u}_{i}$ on the annular regions

$$
u_{i}^{-1}\left(B_{p}^{2 n}\left(\varepsilon_{i, \text { out }}\right)\right) \backslash u_{i}^{-1}\left(B_{p}^{2 n}\left(\varepsilon_{i, \text { in }}\right)\right)=C_{i,-} \cup C_{i,+},
$$

where both $C_{i \pm}$ are of cylindrical type. We prove the following result whose proof duplicates the one from [FOOO2] used in a similar context.

Proposition 13.1. Let $\left(u_{-}, u_{+}\right)$be a Floer trajectory with immersed nodes as before and suppose $u_{i}$ converges to $\left(u_{-}, u_{+}\right)$in level 0 and let $C_{i, \pm}$ be as above. Suppose that $\varepsilon_{i, \text { out }}, \varepsilon_{i \text {,in }}$ are chosen so that (13.3) holds. Then we have

$$
\bmod \left(C_{i, \pm}\right) \rightarrow \infty
$$

Proof. Since both cases are essentially the same, we will just treat the case of $C_{i,+}=C_{i}$.

Since $u_{i}$ are immersed on $C_{i}$, the image $u_{i}\left(C_{i}\right)$ carries the metric $g_{\text {ind }}$ induced from the compatible metric $g_{J}$ on $M$. We denote by $g_{0}$ on $\left[\log \varepsilon_{i, \text { in }}, \log \varepsilon_{i, \text { out }}\right] \times[0,2 \pi]$ the standard product metric. Using Proposition 12.3 , we can find a diffeomorphism

$$
\Phi_{i}: u_{i}\left(C_{i,+}\right) \rightarrow\left[\log \varepsilon_{i, \text { in }}, \log \varepsilon_{i, \text { out }}\right] \times[0,2 \pi]
$$

so that

(1) $\Phi_{i}\left(u_{i}\left(C_{i}\right) \cap\{s\} \times S^{2 n-1}\right)=\{s\} \times[0,2 \pi]$.

(2) For each sufficiently small $\varepsilon>0$, we have

$$
\left|\left(\Phi_{i}\right)_{*}\left(g_{\text {ind }}\right)-g_{0}\right|_{C^{1}}<\varepsilon
$$

on $\left[\log \varepsilon_{i, \text { in }}, \log \varepsilon_{i, \text { out }}\right] \times S^{1}$ for all sufficiently large $i$.

Let $\psi_{j,+}:\left[-L_{i+}, L_{i+}\right] \times S^{1} \rightarrow C_{i+}$ be the orientation preserving conformal diffeomorphism such that

$$
\psi_{i+}\left(\left\{ \pm L_{i+}\right\} \times S^{1}\right) \subset \partial_{ \pm} C_{i} .
$$




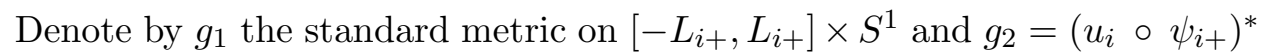
$g_{\text {ind }}$. Since $u_{i} \circ \psi_{i+}$ is pseudo-holomorphic and so

$$
u_{i} \circ \psi_{i+}:\left(\left[-L_{i+}, L_{i+}\right] \times S^{1}, g_{1}\right) \rightarrow\left(u_{i}\left(C_{i}\right), g_{\text {ind }}\right)
$$

is conformal, we have $f^{2} g_{1}=\left(u_{i} \circ \psi_{i+}\right)^{*} g_{\text {ind }}$ and so

$$
g_{1}=f^{2} g_{2}
$$

where $f:\left[-L_{i+}, L_{i+}\right] \times S^{1} \times S^{1} \rightarrow \mathbb{R}$ is a positive smooth function.

We compute

$$
\begin{aligned}
& \left(\int_{\left[-L_{i+}, L_{i+}\right] \times S^{1}} f \Omega_{g_{2}}\right)^{2} \leq\left(\int_{\left[-L_{i+}, L_{i+}\right] \times S^{1}} f^{2} \Omega_{g_{2}}\right)\left(\int_{\left[-L_{i+}, L_{i+}\right] \times S^{1}} \Omega_{g_{2}}\right) \\
& \quad \leq \operatorname{Area}\left(\left[-L_{i+}, L_{i+}\right] \times S^{1} ; g_{1}\right) \\
& \quad \times(1+\varepsilon)\left(\int_{\left[\log \varepsilon_{i, \text { in }}, \log \varepsilon_{i, \text { out }}\right] \times S^{1}} \Omega_{g_{0}}\right) \\
& \leq\left((2 \pi) \cdot\left(2 L_{i+}\right)\right) \cdot(1+\varepsilon) \cdot\left((2 \pi)\left(\log \varepsilon_{i, \text { out }}-\log \varepsilon_{i, \text { in }}\right)\right) \\
& =\left((2 \pi) \cdot\left(2 L_{i+}\right)\right) \cdot(1+\varepsilon)(2 \pi) \cdot\left(\log \varepsilon_{i, \text { out }}-\log \varepsilon_{i, \text { in }}\right) .
\end{aligned}
$$

On the other hand, we derive

$$
\int_{\left[-L_{i+}, L_{i+}\right] \times S^{1}} f \Omega_{g_{2}} \geq(1+\varepsilon)^{-1} \int_{\log \varepsilon_{i, \text { in }}}^{\log \varepsilon_{i, \text { out }}} \operatorname{leng}_{g_{0}}\left(u_{i}^{-1} \circ \gamma_{i, s}\right) d s .
$$

Since the winding number of the curve $u_{i}^{-1} \circ \gamma_{i, s}$ is one and $\gamma_{i, s} \rightarrow \gamma_{a^{+}}$, we have

$$
\operatorname{leng}_{g_{0}}\left(u_{i}^{-1} \circ \gamma_{i, s}\right) \rightarrow 2 \pi
$$

as $i \rightarrow \infty$. Hence, we have proved

$$
\int_{\left[-L_{i+}, L_{i+}\right] \times S^{1}} f \Omega_{g_{2}} \geq(1+\varepsilon)^{-1}\left(\log \varepsilon_{i, \text { out }}-\log \varepsilon_{i, \text { in }}\right) \times 2 \pi .
$$

Substituting this into (13.4), we obtain

$$
\begin{aligned}
& \left((1+\varepsilon)^{-1}\left(\log \varepsilon_{i, \text { out }}-\log \varepsilon_{i, \text { in }}\right) \times 2 \pi\right)^{2} \\
& \quad \leq\left((2 \pi) \cdot\left(2 L_{i+}\right)\right) \times(1+\varepsilon)\left((2 \pi) \cdot\left(\log \varepsilon_{i, \text { out }}-\log \varepsilon_{i, \text { in }}\right)\right.
\end{aligned}
$$

and so

$$
\log \varepsilon_{i, \text { out }}-\log \varepsilon_{j, \text { in }} \leq(1+\varepsilon) 2 L_{i+} .
$$

This proves $L_{i+} \rightarrow \infty$ as $i \rightarrow \infty$ since we have chosen $\varepsilon_{i, \text { out }}, \varepsilon_{i, \text { in }}$ so that

$$
\left|\log \varepsilon_{i, \text { out }}-\log \varepsilon_{i, \text { in }}\right| \rightarrow \infty .
$$


Next, we recall the definition of the energy that Hofer introduced in $[\mathbf{H o}]$, which we denote by $E_{\Sigma}$ and $E_{d \lambda}$ restricted to the case of $\mathbb{C}^{n} \backslash\{0\}$ with the standard symplectic form $\omega_{0}$. If we denote by $\lambda$ the standard contact form on $S^{2 n-1}(1) \subset \mathbb{C}^{n}$ and by $(r, \Theta)$ the polar coordinates of $\mathbb{C}^{n} \cong \mathbb{R}_{+} \times S^{2 n-1}(1)$, then we have

$$
\omega_{0}=d\left(r \Theta^{*} \lambda\right)
$$

Using the diffeomorphism $\mathbb{R} \rightarrow \mathbb{R}_{+} ; s \mapsto \mathrm{e}^{s}$, we identify $\mathbb{C}^{n} \backslash\{0\}$ with $\mathbb{R} \times S^{2 n-1}$. Then pull-back of the standard complex structure $J_{0}$ on $\mathbb{C}^{n} \backslash\{0\}$ is invariant under the translation of $\mathbb{R}$-direction on $\mathbb{R} \times S^{2 n-1}$ as well as $\Theta^{*} d \lambda$ and $\Theta^{*} \lambda$.

We will pull-back the symplectic form $\omega$ on $M$ by the map $\exp _{p}^{I}:=I^{-1}$ to $\omega_{0}$ by a Darboux chart $I$ near the nodal point $p \in M$ such that $I^{*} J(0)=$ $J(p)$. The following lemma is immediate whose proof is omitted.

Lemma 13.1. Let $\widetilde{J}_{\varepsilon}$ be the almost complex structure on $\left(T_{p} M, \omega_{p}\right) \cong$ $\left(\mathbb{C}^{n}, \omega_{0}\right)$ defined by $\widetilde{J}_{\varepsilon}=\left(\exp _{p}^{I} \circ R_{\varepsilon}\right)^{*} J$. Then there exists $\varepsilon_{0}>0$ such that we have

$$
\left|\widetilde{J}_{\varepsilon}(x)-J_{p}\right| \leq C \varepsilon|x|
$$

for all $|x| \leq \varepsilon_{0}$ where $|\cdot|$ is the norm induced by the standard metric on $\mathbb{C}^{n} \cong T_{p} M$. In particular, we have

$$
\left|\widetilde{J}_{\varepsilon_{i}}(x)-J_{p}\right| \leq C \delta_{i}
$$

for all $x \in \frac{1}{\varepsilon_{i}} B_{p}^{2 n}\left(\delta_{i}\right) \cong B_{p}^{2 n}\left(\delta_{i} / \varepsilon_{i}\right) \subset T_{p} M$ for any $0<\delta_{i} \leq \varepsilon_{0}$.

Now we introduce the following

Definition 13.2 ( $d \lambda$-energy). Let $\Sigma$ be a compact surface with boundary and let $u: \Sigma \rightarrow \mathbb{C}^{n} \backslash\{0\}$. We define the $d \lambda$-energy, denoted by $E_{d \lambda}$ by

$$
E_{d \lambda}(u)=\int_{\Sigma}(\Theta \circ u)^{*} d \lambda .
$$

We also use another energy denoted by $E_{\Sigma}[\mathbf{H o}]$. Consider the interval $[a, b] \subset \mathbb{R}$ and let $\mathcal{C}=\mathcal{C}_{[a, b]}$ be the set of smooth functions

$$
\rho:(a, b) \rightarrow[0,1]
$$

such that

(1) supp $\rho$ is of compact support,

(2) $\int_{a}^{b} \rho(u) d u=1$.

Then we consider its integral, denoted by $\widetilde{\rho}$,

$$
\widetilde{\rho}(s)=\int_{a}^{s} \rho(u) d u \text {. }
$$

Composing $\widetilde{\rho}$ with the projection to the $\mathbb{R}$-direction, we regard $\widetilde{\rho}$ as a function on $\mathbb{R} \times S^{2 n-1} \cong \mathbb{C}^{n} \backslash B^{2 n}(1)$. Note that $\widetilde{\rho} \equiv 0$ near the lower limit $s=a$ and $\widetilde{\rho} \equiv 1$ near the upper limit $s=b$. 
Definition 13.3. Let $\rho \in \mathcal{C}$ and $\widetilde{\rho}$ as above. We define $E_{\Sigma}(u ;(a, b))$ by

$$
E_{\Sigma}(u ;(a, b))=\sup _{\rho \in \mathcal{C}} \int_{\Sigma} u^{*} d\left(\widetilde{\rho} \widetilde{\Theta}^{*} \lambda\right) .
$$

We now prove the following.

Lemma 13.2. Denote $C_{i}=u_{i}^{-1}\left(B_{p}\left(\varepsilon_{i, \text { out }}\right) \backslash B_{p}\left(\varepsilon_{i, \text { in }}\right)\right)$ and consider the restriction of $u_{i}$ on $C_{i}$. We have

$$
\lim _{i \rightarrow \infty} E_{d \lambda ; C_{i}}\left(\widetilde{u}_{i}\right)=0
$$

and in particular $E_{d \lambda ; C_{i}}\left(\widetilde{u}_{i}\right)$ is uniformly bounded.

Proof. Note that $C_{i}$ has decomposition

$$
C_{i}=C_{i, 1} \cup C_{i,+}:
$$

$C_{i,-}, C_{i,+}$ are surfaces of annular type such that

$$
\begin{aligned}
& \partial C_{i,-}=\partial_{+} C_{i,-} \cup \partial_{-} C_{i,-}, \\
& \partial C_{i,+}=\partial_{+} C_{i,+} \cup \partial_{-} C_{i,+},
\end{aligned}
$$

where $\widetilde{u}_{i}\left(\partial_{+} C_{i}\right) \subset \partial B^{2 n}\left(\varepsilon_{i, \text { out }} / \varepsilon_{i}\right)$ and $\widetilde{u}_{i}\left(\partial_{-} C_{i}\right) \subset \partial B^{2 n}\left(\varepsilon_{i, \text { out }} / \varepsilon_{i}\right)$.

Since both cases can be treated the same, we will focus on $C_{i,-}$. By Stokes' formula, we obtain

$$
E_{d \lambda}\left(\widetilde{u}_{i}\right)=\int_{C_{i,-}}\left(\Theta \circ \widetilde{u}_{i}\right)^{*} d \lambda=\int_{\partial_{+} C_{i,-}}\left(\Theta \circ \widetilde{u}_{i}\right)^{*} \lambda-\int_{\partial_{-} C_{i,-}}\left(\Theta \circ \widetilde{u}_{i}\right)^{*} \lambda .
$$

Proposition 12.3 implies

$$
\begin{aligned}
& \left.\lim _{i \rightarrow \infty} \Theta \circ \widetilde{u}_{i}\right|_{\partial_{+} C_{i,-}}=\gamma_{-}, \\
& \left.\lim _{i \rightarrow \infty} \Theta \circ \widetilde{u}_{i}\right|_{\partial_{-} C_{i,-}}=\gamma_{-},
\end{aligned}
$$

where $\gamma_{-}$is the Reeb orbit of $S^{2 n-1}$ comes from the tangent cone of $u_{-}$at the node. This implies $\lim _{i \rightarrow \infty} E_{d \lambda ; C_{i,-}}\left(\widetilde{u}_{i}\right)=0$.

Since the same argument applies to $C_{i,+}$ if we replace $\gamma_{-}$by $\gamma_{+}$, we have proved

$$
\lim _{i \rightarrow \infty} E_{d \lambda ; C_{i}}\left(\widetilde{u}_{i}\right)=0
$$

Next we study $E_{C_{i}}\left(\widetilde{u}_{i}\right)=E_{C_{i}}\left(\widetilde{u}_{i} ;\left(\log \left(\varepsilon_{i, \text { out }} / \varepsilon_{i}\right), \log \left(\varepsilon_{i, \text { in }} / \varepsilon_{i}\right)\right)\right.$.

Lemma 13.3. For any given $\delta>0$, there exists $N=N(\delta)$ such that

$$
E_{C_{i}}\left(\widetilde{u}_{i}\right)<2 \pi+\delta
$$

for all $i \geq N$. 
Proof. Let $\rho \in \mathcal{C}$ and $\widetilde{\rho}$ be the associated integral

$$
\widetilde{\rho}(s)=\int_{0}^{s} \rho(u) d u .
$$

Noting that $\widetilde{\rho} \equiv 0$ near $\partial_{-} C_{i}$, we use Stokes' theorem to show

$$
\int_{C_{i}} \widetilde{u}_{i}^{*} d\left(\widetilde{\rho} \Theta^{*} \lambda\right)=\int \gamma_{i,-, \text { out }}^{*} \lambda+\int \gamma_{i,+, \text { out }}^{*} \lambda
$$

where $\gamma_{i, \pm \text { out }}=\left.\widetilde{u}_{i}\right|_{\partial_{+} C_{i, \pm}}$. Proposition 12.3 implies that $\gamma_{i, \pm, \text { out }} \rightarrow \gamma_{ \pm}$ respectively and so each term converges to $2 \pi$ as $i \rightarrow \infty$. Hence there exists $N \in \mathbb{Z}_{+}$such that if $i \geq N$,

$$
\int_{C_{i, \pm}} \widetilde{u}_{i}^{*} d\left(\widetilde{\rho} \Theta^{*} \lambda\right) \leq 2 \pi+\delta
$$

for any $\rho \in \mathcal{C}$. Fixing any such $N$ and taking the supremum over $\rho \in \mathcal{C}$, we have proved $E_{\Sigma}\left(\widetilde{u}_{i}\right) \leq 2 \pi+\delta$ for all $i$.

Now we take a conformal parameterization $\varphi_{i, \pm}:\left[-L_{i, \pm}, L_{i, \pm}\right] \times S^{1} \cong C_{i, \pm}$ and consider the composition $\widetilde{u}_{i} \circ \varphi_{i, \pm}=: v_{i, \pm}$. Proposition 13.1 implies $L_{i,+} \rightarrow \infty$ as $i \rightarrow \infty$ and Lemma 13.2 implies

$$
\lim _{i \rightarrow \infty} \int_{-L_{i, \pm}}^{L_{i, \pm}} \int_{S^{1}} v_{i, \pm}^{*} \Theta^{*} d \lambda=0, \quad E_{\Sigma}\left(v_{i, \pm}\right) \leq 2 \pi+\delta .
$$

Once we have these energy bounds and Theorem 13.2, the argument from [Ho, HWZ4] imply the following proposition when applied to $C_{i,-}$ and of $C_{i,+}$ (see also Chapter 10 of [FOOO2].)

Proposition 13.2. Let $\Sigma_{i}^{\prime}=C_{i,-} \cup C_{i,+}$ be the decomposition mentioned before, and let $v_{i, \pm}$ be the above map restricted to one of the two components respectively. Then the sequence $v_{i, \pm}$ converge to holomorphic cylinders $\widetilde{u}_{\infty, \pm}$ : $\mathbb{R} \times S^{1} \rightarrow \mathbb{R} \times S_{p}^{2 n-1} \cong \mathbb{C}^{n} \backslash\{0\}$ with

$$
\widetilde{u}_{\infty, \pm}=\left(s \circ \widetilde{u}_{\infty, \pm}, \Theta \circ \widetilde{u}_{\infty, \pm}\right)
$$

given by

$$
s \circ \widetilde{u}_{\infty, \pm}\left(\tau^{\prime}, t^{\prime}\right)=\left(2 \pi \tau+s_{ \pm}, \gamma\left(2 \pi t+\theta_{ \pm}\right)\right)=: u_{a_{ \pm}, s_{ \pm}}^{\mathrm{flat}}
$$

for the real numbers $s_{ \pm}$and $\theta_{ \pm}$, where $\gamma_{ \pm}$are the Reeb orbit associated to the tangent cone of $u_{-}$or $u_{+}$, respectively, on $C_{i,-}$ on $C_{i,+}$.

13.2. Convergence in the central region. Now we focus our attention on the central region

$$
\mathcal{U}_{i, \text { int }}:=u_{i}^{-1}\left(B_{p}^{2 n}\left(\delta_{i}\right)\right) .
$$

By the convergence proved in Theorem 11.1, there exists $\delta_{i} \rightarrow 0$ such that

$$
u_{i}\left(\mathcal{U}_{i, i n t}\right) \subset B_{p}^{2 n}\left(\delta_{i}\right)
$$


and $u_{i}$ satisfies $\bar{\partial}_{J} u_{i}=0$ near $u_{i}^{-1}\left(\partial B_{p}^{2 n}\left(\delta_{i}\right)\right)$. We may choose $\varepsilon_{i, \text { in }}$ and $\varepsilon_{i, \text { out }}$ so that

$$
\varepsilon_{i, \text { in }}<\delta_{i}<\varepsilon_{i, \text { out }}<\varepsilon_{0} .
$$

We denote $\Sigma_{i}^{\prime \prime}=u_{i}^{-1}\left(B_{p}^{2 n}\left(\delta_{i}\right)\right)$. Then we have the maps $\widetilde{u}_{i}$ that satisfy

$$
\widetilde{u}_{i}\left(\Sigma_{i}^{\prime \prime}\right) \subset B_{p}^{2 n}\left(\delta_{i} / \varepsilon_{i}\right), \quad \widetilde{u}_{i}\left(\partial \Sigma_{i}^{\prime \prime}\right) \subset \partial B_{p}^{2 n}\left(\delta_{i} / \varepsilon_{i}\right) .
$$

In terms of the orientation convention provided in Definition 13.1, both boundaries of $\Sigma_{i}^{\prime \prime}$ are outside boundaries.

We again consider the rescaled maps $\widetilde{u}_{i}: \Sigma_{i}^{\prime \prime} \rightarrow T_{p} M \cong \mathbb{C}^{n}$ given by

$$
\widetilde{u}_{i}(z)=\frac{1}{\varepsilon_{i}}\left(\exp _{p}^{I}\right)^{-1} \circ u_{i}(z) .
$$

By definition of $\widetilde{J}_{\varepsilon}$, this map satisfies

$$
\left(d \widetilde{u}_{i}+R_{\varepsilon_{i}}^{*} P_{\varepsilon_{i} f}\left(\widetilde{u}_{i}\right)\right)_{\widetilde{J}_{\varepsilon_{i}}}^{(0,1)}=0,
$$

where $R_{\varepsilon}: \mathbb{C}^{n} \rightarrow \mathbb{C}^{n}$ is the rescaling map $x \mapsto \varepsilon x$ on $\mathbb{C}^{n}$.

The following lemma is immediate to check whose proof is omitted.

Lemma 13.4. We can rewrite (13.7) as

$$
\bar{\partial}_{\widetilde{J}_{\varepsilon_{i}}} \widetilde{u}_{i}+P_{\vec{a}}\left(\widetilde{u}_{i}\right)_{J_{p}}^{(0,1)}=C_{\varepsilon_{i}}\left(\widetilde{u}_{i}\right) \cdot \widetilde{u}_{i}
$$

where $\vec{a}=\nabla f(p), C_{\varepsilon_{i}}\left(\widetilde{u}_{i}\right)$ is the obvious operator and we have

$$
\left|\widetilde{J}_{\varepsilon_{i}}\left(\widetilde{u}_{i}\right)-J_{p}\right| \leq C \varepsilon_{i}\left|u_{i}\right|, \quad\left|C_{\varepsilon_{i}}\left(\widetilde{u}_{i}\right) \widetilde{u}_{i}\right| \leq C \delta_{i}
$$

as long as $|u| \leq \delta_{i} / \varepsilon_{i}$.

We now examine the left-hand side of (13.8). We conformally parameterize $\Sigma_{i}^{\prime \prime} \cong\left[-L_{i}, L_{i}\right] \times S^{1}$ with conformal coordinates denoted by $\left(\tau^{\prime}, t^{\prime}\right)$. Then we prove the following lemma by the same way as Proposition 13.1.

Lemma 13.5. Let $\bmod \left(\Sigma_{i}^{\prime \prime}\right)$ be the conformal modulus of $\Sigma_{i}^{\prime \prime}$ as defined above. Then $\bmod \left(\Sigma_{i}^{\prime \prime}\right) \rightarrow \infty$.

We can write

$$
\widetilde{u}_{i}\left(\tau^{\prime}, t^{\prime}\right)=-\tau^{\prime} \vec{a}+\xi_{i}\left(\tau^{\prime}, t^{\prime}\right)
$$

at least as long as $|\tau \vec{a}|<\delta_{i} / \varepsilon_{i}$, or equivalently for $\tau$ satisfying

$$
|\tau| \leq \frac{\delta_{i}}{\varepsilon_{i}|\vec{a}|}
$$

With this conformal coordinate, we can write

$$
\left(\bar{\partial}_{\widetilde{J}_{\varepsilon_{i}}} \widetilde{u}_{i}+P_{\vec{a}}\left(\widetilde{u}_{i}\right)_{J_{p}}^{(0,1)}\right)\left(\frac{\partial}{\partial \tau^{\prime}}\right)=\frac{\partial \widetilde{u}_{i}}{\partial \tau^{\prime}}+\widetilde{J}_{\varepsilon_{i}} \frac{\partial \widetilde{u}_{i}}{\partial t^{\prime}}+\vec{a}=\frac{\partial \xi}{\partial \tau^{\prime}}+\widetilde{J}_{\varepsilon_{i}} \frac{\partial \xi}{\partial t^{\prime}} .
$$


Therefore (13.8) is equivalent to

$$
\frac{\partial \xi_{i}}{\partial \tau^{\prime}}+\widetilde{J}_{\varepsilon_{i}} \frac{\partial \xi_{i}}{\partial t^{\prime}}=C_{\varepsilon_{i}}\left(\widetilde{u}_{i}\right)\left(\frac{\partial}{\partial \tau^{\prime}}\right) \cdot \widetilde{u}_{i}
$$

In particular, we derive

$$
\left|\frac{\partial \xi_{i}}{\partial \tau^{\prime}}+\widetilde{J}_{\varepsilon_{i}} \frac{\partial \xi_{i}}{\partial t^{\prime}}\right| \leq C \delta_{i}
$$

from (13.9) on $B^{2 n}\left(\delta_{i} / \varepsilon_{i}\right)$. Therefore, if we prove that $\xi_{i}$ (or equivalently $\widetilde{u}_{i}$ ) converges locally in $C^{1}$-topology, then the limit of $\xi_{i}$ must be holomorphic and hence the local limit of $\widetilde{u}_{i}$ will have the form

$$
-\tau^{\prime} \vec{a}+\xi_{\infty}\left(\tau^{\prime}, t^{\prime}\right), \quad \text { with } \bar{\partial} \xi_{\infty}=0
$$

as we are expecting. We will now prove this convergence.

Consider the energies of $\widetilde{u}_{i}$ given by

$$
E_{\text {int }}\left(\widetilde{u}_{i}\right)=\int_{\left\{\left.z \in \Sigma_{i}^{\prime \prime}|| \widetilde{u}_{i}(z)\right|_{\left.\mathbb{C}^{n} \leq 4\right\}}\right.} \widetilde{u}_{i}^{*} d\left(\mathrm{e}^{2 s} \lambda\right)
$$

and

$$
E_{d \lambda}\left(\widetilde{u}_{i} ; S\right):=\int_{\left\{\left.z \in \Sigma_{i}^{\prime \prime}|| \widetilde{u}_{i}(z)\right|_{\left.\left.\mathbb{C}^{n} \geq e^{S}\right)\right\}}\right.} \widetilde{u}_{i}^{*} d \lambda .
$$

Next let $\mathcal{C}$ be the set of all nonnegative smooth function $\rho: \mathbb{R} \rightarrow \mathbb{R}$ whose support is compact and is contained in $[2, \infty)$ and such that $\int \rho(s)=1$, and $\widetilde{\rho}$ be the function defined by

$$
\widetilde{\rho}(s)=\int_{2}^{s} \rho(u) d u \text {. }
$$

Then we define

$$
E_{\text {neck }}\left(\widetilde{u}_{i}\right)=\sup _{\rho \in \mathcal{C}} \int \widetilde{u}_{i}^{*} d(\widetilde{\rho} \lambda) .
$$

Lemma 13.6. $E_{\text {neck }}\left(\widetilde{u}_{i}\right)$ and $E_{\text {int }}\left(\widetilde{u}_{i}\right)$ are uniformly bounded above over $i$.

Proof. We recall the energy $E_{\Sigma}\left(u_{i} ;\left[\log \varepsilon_{i}, \log \delta_{i}\right]\right)$ from Definition 13.3 over those $\rho$ defined on $\left[\log \varepsilon_{i}, \log \delta_{i}\right]$. Then by the same proof as Lemma 13.3, we have the uniform upper bound

$$
E_{\Sigma}\left(u_{i} ;\left[\log \varepsilon_{i}, \log \delta_{i}\right]\right)<C
$$

for some $C$ independent of $i$. It is easy to see from the scaling property that

$$
E_{\text {neck }}\left(\widetilde{u}_{i}\right) \leq E\left(u_{i}\right)
$$

and hence $E_{\text {neck }}\left(\widetilde{u}_{i}\right)$ is uniformly bounded.

On the other hand, we have

$$
E_{\text {int }}\left(\widetilde{u}_{i}\right) \leq \varepsilon_{i}^{-2} \int_{u_{i}^{-1}\left(B_{p}^{2 n}\left(2 \varepsilon_{i}\right)\right)} u_{i}^{*} \omega_{0}
$$


by definition. But Stokes' formula gives rise to

$$
\begin{aligned}
\int_{u_{i}^{-1}\left(B_{p}^{2 n}\left(2 \varepsilon_{i}\right)\right)} u_{i}^{*} \omega_{0} & =\int_{u_{i}^{-1}\left(B_{p}^{2 n}\left(2 \varepsilon_{i}\right)\right)} u_{i}^{*} d\left(r^{2} \Theta^{*} \lambda\right) \\
& =\int_{u_{i}^{-1}\left(\partial B_{p}^{2 n}\left(2 \varepsilon_{i}\right)\right)}\left(2 \varepsilon_{i}\right)^{2} \Theta^{*} \lambda \cong 4 \varepsilon_{i}^{2}(2 \pi+2 \pi)
\end{aligned}
$$

by the immersion property of the node and the $\varepsilon_{i}$-controlled convergence of $u_{i}$ to $\left(u_{-}, u_{+}, u_{0}\right)$ mentioned in the previous section. This finishes the proof.

We also prove the following lemma in the same way as Lemma 13.2.

Lemma 13.7. We have

$$
\lim _{S \rightarrow \infty} \limsup _{i \rightarrow \infty} E_{d \lambda}\left(\widetilde{u}_{i} ; S\right)=0 .
$$

We can obtain the same kind of estimates for $\xi_{i}=\widetilde{u}_{i}+\tau a$ from the identity

$$
d \xi_{i}=d \widetilde{u}_{i}+a d \tau .
$$

\section{Lemma 13.8.}

$$
\begin{gathered}
\lim _{i \rightarrow 0} E_{\text {int }}\left(\widetilde{u}_{i}\right)=\lim _{i \rightarrow 0} E_{\text {int }}\left(\xi_{i}\right)=4^{3} \pi, \\
\lim _{i \rightarrow 0}\left|E_{d \lambda}\left(\widetilde{u}_{i} ; S\right)-E_{d \lambda}\left(\xi_{i} ; S\right)\right|=0, \\
\lim _{i \rightarrow \infty}\left|E_{\text {neck }}\left(\widetilde{u}_{i}\right)-E_{\text {neck }}\left(\xi_{i}\right)\right|=0 .
\end{gathered}
$$

Proof. The proofs for $E_{d \lambda}$ and $E_{\text {int }}$ are similar. We will just prove the identity for $E_{\text {int }}$. By definition, we have

$$
\begin{aligned}
E_{\text {int }}\left(\widetilde{u}_{i}\right) & =\int_{\Sigma_{i}^{\prime \prime} \cap \widetilde{u}_{i}^{-1}\left(B^{2 n}(4)\right)} \widetilde{u}_{i}^{*} d\left(r^{2} \lambda\right) \\
& =4^{2} \int_{\partial\left(\Sigma_{i}^{\prime \prime} \cap \widetilde{u}_{i}^{-1}\left(B^{2 n}(4)\right)\right)}\left(\partial \widetilde{u}_{i}\right)^{*} \lambda \rightarrow 4^{2}\left(\int \gamma_{+}^{*} \lambda+\int \gamma_{-}^{*} \lambda\right) \\
& =4^{2} 4 \pi=4^{3} \pi .
\end{aligned}
$$

Here we again used the immersion property of nodes and the fact that both ends of the cylinder are positive. The same applies to $\xi_{i}$ because $\lim _{i \rightarrow \infty}|\vec{a}| /\left|\frac{\partial \widetilde{u}_{i}}{\partial \tau}\right| \rightarrow 0$

Next we examine $E_{\text {neck }}$. For each $\rho \in \mathcal{C}$, we evaluate

$$
\int \widetilde{u}_{i}^{*} d(\widetilde{\rho} \lambda)=\int_{\partial B^{2 n}\left(\delta_{i}\right)}\left(\partial_{+} \widetilde{u}_{i}\right)^{*} \lambda
$$

where $\partial_{+} \widetilde{u}_{i}:=\left.\widetilde{u}_{i}\right|_{\partial_{+}}$and $\partial_{+}$is the outside boundary of $\widetilde{u}_{i}^{-1}\left(\partial B^{2 n}\left(\delta_{i}\right)\right)$. Therefore, we have obtained

$$
E_{\text {neck }}\left(\widetilde{u}_{i}\right)-E_{\text {neck }}\left(\xi_{i}\right)=\int_{\partial B^{2 n}\left(r_{\text {out }}\right)}\left(\left(\partial_{+} \widetilde{u}_{i}\right)^{*} \lambda-\left(\partial_{+} \xi_{i}\right)^{*} \lambda\right)
$$


for all $\rho \in \mathcal{C}$. As $i \rightarrow \infty$, the conformal coordinates $\left(\tau^{\prime}, t^{\prime}\right)$ of the domain $C_{i, \text { int }}:=\widetilde{u}_{i}^{-1}\left(B^{2 n}\left(\delta_{i}\right) \cong\left[-L_{i}^{\prime}, L_{i}^{\prime}\right] \times S^{1}\right.$ converges to the given coordinates $(\tau, t)$ near $\tau^{\prime}=L_{i}^{\prime}$, it follows that we have

$$
\left|\frac{\partial \tau}{\partial t^{\prime}}\right| \leq C
$$

near $L_{i}^{\prime}$ as $i \rightarrow \infty$ and so $|a \tau|_{C^{1} ; \partial C_{i}} \rightarrow 0$ in the cylindrical metrics of the domain and the target. Therefore, it follows:

$$
\left|\widetilde{u}_{i}-\xi_{i}\right|_{C^{1} ; \partial C_{i, \text { int }}} \rightarrow 0
$$

in the cylindrical metric. We note that this convergence is uniform over $\rho \in \mathcal{C}$ as long as supp $\rho$ is contained in a ball $B^{2 n}(r)$ of common radius $r>0$. Furthermore the convergence of $\widetilde{u}_{i}\left( \pm L_{i}^{\prime}, t\right) \rightarrow \gamma_{ \pm}$as $L_{i}^{\prime} \rightarrow \infty$. Combining all these, we obtain

$$
\lim _{i \rightarrow \infty}\left|E_{\text {neck }}\left(\widetilde{u}_{i}\right)-E_{\text {neck }}\left(\xi_{i}\right)\right|=0 .
$$

This finishes the proof.

We note that both $E_{d \lambda}$ and $E_{\text {neck }}$ are invariant under the automorphisms of $\mathbb{C}^{n}$, i.e., under homothety and translations. By applying a suitable sequence of automorphisms $g_{v_{i}, \lambda_{i}}$ to $\xi_{i}$ we can achieve

$$
\min _{t \in S^{1}}\left|g_{v_{i}, \lambda_{i}} \circ \xi_{i}(0, t)\right|=1
$$

for all $i$.

We now prove the following derivative bound.

Proposition 13.3. Denote $\bar{\xi}_{i}=g_{v_{i}, \lambda_{i}} \circ \xi_{i}$. For each $L$, there exists a constant $C=C(L)$ such that

$$
\sup _{-L \leq\left|\tau^{\prime}\right| \leq L}\left|d \xi_{i}\left(\tau^{\prime}, t^{\prime}\right)\right|<C(L) .
$$

Proof. The proof will be given by a bubbling-off analysis which is a variation of the proof of Proposition 27 [Ho]. Suppose to the contrary that there exists a sequence $z_{k} \in\left[-R_{0}, R_{0}\right] \times S^{1} \subset \Sigma_{k}^{\prime \prime} \cong\left[-L_{k}, L_{k}\right] \times S^{1}$ with $L_{k} \rightarrow \infty$ such that

$$
\left|d \bar{\xi}_{k}\left(z_{k}\right)\right| \rightarrow \infty
$$

The following is from $[\mathbf{H V}, \mathbf{F O O O 2}]$.

Lemma 13.9 (Lemma 62.149, [FOOO2]). There exists another sequence $z_{i}^{\prime} \in\left[-R_{0}-1, R_{0}+1\right] \times S^{1}$ satisfying the following properties:

(1) $\left|d \bar{\xi}_{i}\left(z_{i}^{\prime}\right)\right|:=C_{i} \rightarrow \infty$

(2) If $d_{g_{\mathbb{C}}^{\prime}}\left(z^{\prime}, z_{i}^{\prime}\right) \leq C_{i}^{-1 / 2}$ for $z^{\prime} \in \mathbb{C}$, then $\left|d \bar{\xi}_{i}\right|_{g_{\mathbb{C}}^{\prime}, g_{\mathbb{C}^{n}}^{\prime}} \leq 2 C_{i}$.

The following is a verbatim translation of Lemma 62.151 [FOOO2] in our context. For readers' convenience, we duplicate it therefrom with minor modifications. 
Lemma 13.10 (Lemma 62.151, [FOOO2]). The sequence $\bar{\xi}_{i}\left(z_{i}^{\prime}\right) \in \mathbb{C}^{n}$ is bounded.

Proof. The proof is by contradiction. Suppose to the contrary that

$$
R_{3, i}=\left|\bar{\xi}_{i}\left(z_{i}^{\prime}\right)\right|_{\mathbb{C}^{n}} \rightarrow \infty .
$$

We put

$$
\begin{aligned}
D_{i} & =\left\{u \in \mathbb{C} \mid \operatorname{dist}_{g_{\mathbb{H}}^{\prime}}\left(C_{i}^{-1} u+z_{i}^{\prime}, z_{i}^{\prime}\right)\right. \\
& \left.<\min \left\{C_{i}^{-1} \sqrt{R_{3, i}} / 2, C_{i}^{-1 / 2}\right\}, C_{i}^{-1} u+z_{i}^{\prime} \in \mathbb{H}\right\} .
\end{aligned}
$$

We note that $D_{i}$ is a convex domain of its diameter with the order of

$$
\min \left\{\sqrt{R_{3, i}} / 2, C_{i}^{1 / 2}\right\},
$$

which goes to $\infty$ as $i \rightarrow \infty$ by the hypotheses.

We define $\widetilde{\xi}_{i}: D_{i} \rightarrow \mathbb{C}^{n}$ by

$$
\widetilde{\xi}_{i}(u)=\bar{\xi}_{i}\left(C_{i}^{-1} u+z_{i}^{\prime}\right) .
$$

Then we have

$$
\left|d \widetilde{\xi}_{i}\left(z_{i}\right)\right| \geq 1
$$

We now prove

$$
\inf _{u \in D_{i}}\left|\widetilde{\xi}_{i}(u)\right| \geq \sqrt{R_{3, i}}\left(\sqrt{R_{3, i}}-1\right)>2 S_{0}
$$

if $i$ is sufficiently large. We note

$$
\begin{aligned}
\left|\widetilde{\xi}_{i}(u)\right| & \geq\left|\widetilde{\xi}_{i}(0)\right|-\left|\widetilde{\xi}_{i}(u)-\widetilde{\xi}_{i}(0)\right| \\
& =\left|\xi_{i}\left(z_{i}^{\prime}\right)\right|-\left|\widetilde{\xi}_{i}(u)-\widetilde{\xi}_{i}(0)\right| .
\end{aligned}
$$

We have $\left|\bar{\xi}_{i}\left(z_{i}^{\prime}\right)\right|=R_{3, i}$ and

$$
\begin{aligned}
\left|\widetilde{\xi}_{i}(u)-\widetilde{\xi}_{i}(0)\right| & \leq \int_{0}^{1}\left|u \cdot \nabla \widetilde{\xi}_{i}(s u)\right| d s \\
& =\int_{0}^{1}\left|u \cdot C_{i}^{\prime-1} \nabla \bar{\xi}_{i}\left(C_{i}^{\prime-1}(s u)+z_{i}^{\prime}\right)\right| d s \\
& \leq \int_{0}^{1}\left|C_{i}^{\prime-1} u\right|\left|\nabla \bar{\xi}_{i}\left(C_{i}^{\prime-1}(s u)+z_{i}^{\prime}\right)\right| d s .
\end{aligned}
$$

But since $s u \in D_{i}$ for all $s \in[0,1]$, we have

$$
\operatorname{dist}\left(C_{i}^{\prime-1}(s u)+z_{i}^{\prime}, z_{i}^{\prime}\right) \leq C_{i}^{\prime-1 / 2} .
$$

Then (13.17) implies

$$
\left|\nabla \bar{\xi}_{i}\left(C_{i}^{\prime-1}(s u)+z_{i}^{\prime}\right)\right| \leq 2 C_{i}^{\prime} .
$$

Therefore, we have

$$
\left|\widetilde{\xi}_{i}(u)-\widetilde{\xi}_{i}(0)\right| \leq 2|u| \leq \sqrt{R_{3, i}} .
$$


Substituting these into (13.17), we derive

$$
\left|\widetilde{\xi}_{i}(u)\right| \geq R_{3, i}-\sqrt{R_{3, i}}=\sqrt{R_{3, i}}\left(\sqrt{R_{3, i}}-1\right) .
$$

This finishes the proof of (13.16).

Since $\left(H_{-1}^{\alpha}\right)^{\prime} \cap\left(\mathbb{C}^{n} \backslash B^{2 n}\left(2 S_{0}\right)\right) \subset \mathbb{R}^{n} \cup \Lambda,(13.16)$ allows us to regard $\widetilde{\xi}_{i}$ as a sequence of maps

$$
\widetilde{\xi}_{i}: D_{i} \rightarrow \mathbb{R} \times S^{2 n-1} \cong \mathbb{C}^{n} \backslash \subset \mathbb{C}^{n} .
$$

We derive from Lemma 13.8

$$
E\left(\widetilde{\xi}_{i}\right) \leq E_{0}, \quad E_{d \lambda}\left(\widetilde{\xi}_{i}\right) \rightarrow 0 .
$$

Then we can find $s_{i}^{\prime} \rightarrow \infty$ and a subsequence such that $\mathfrak{T}_{s_{i}^{\prime}} \circ \widetilde{\xi}_{i}$ converges to a map

$$
\widetilde{\xi}_{\infty}: D_{\infty} \rightarrow \mathbb{R} \times S^{2 n-1}
$$

in compact $C^{\infty}$ topology. Therefore, we derive $\left|d \widetilde{\xi}_{\infty}\left(z_{\infty}\right)\right| \geq 1$ from (13.15). But this gives rise to a contradiction, which finishes the proof.

Now we go back to the proof of Proposition 13.3.

Define a new map $\widetilde{v}_{k}: D_{k} \rightarrow \mathbb{C}^{n}$ by

$$
\widetilde{v}_{k}(u)=\bar{\xi}_{k}\left(z_{k}+\frac{u}{C_{k}}\right),
$$

where $D_{k} \subset \mathbb{C}$ is defined by

$$
D_{k}=\left\{u \in \mathbb{C} \mid d_{c y l}\left(z_{k}^{\prime}+u / C_{k}, z_{k}^{\prime}\right)<C_{k}^{-1 / 2}, z_{k}^{\prime}+u / C_{k} \in\left[-L_{k}, L_{k}\right] \times S^{1}\right\} .
$$

Since $z_{k}^{\prime} \in\left[-R_{0}, R_{0}\right] \times S^{1}$, it follows:

$$
\begin{aligned}
z_{k}^{\prime}+u / C_{k} & \in\left[-R_{0}+C_{k}^{-1 / 2}, R_{0}+C_{k}^{-1 / 2}\right] \times S^{1} \\
& \subset\left[-R_{0}-1, R_{0}+1\right] \times S^{1} \subset\left[-L_{k}, L_{k}\right] \times S^{1}
\end{aligned}
$$

and so the map $\widetilde{v}_{k}$ is well defined on $D_{k} \cong B^{2}\left(C_{k}^{1 / 2}\right)$. Then $\widetilde{v}_{k}$ satisfies the following properties:

(1) $\widetilde{v}_{k}(0)=\bar{\xi}_{k}\left(z_{k}\right)$ is bounded,

(2) $E\left(\widetilde{v}_{k}\right)<C$,

(3) $\int_{D_{k}} \widetilde{v}_{k}^{*} \Theta^{*} d \lambda \rightarrow 0$ as $k \rightarrow \infty$

(4) $\left|d \widetilde{v}_{k}(u)\right| \leq 2$ on $D_{k}$ and $\left|d \widetilde{v}_{k}(0)\right|=1$

(5) $\left|\bar{\partial} \widetilde{v}_{k}\right| \rightarrow 0$ as $k \rightarrow \infty$.

Therefore, by taking a diagonal subsequence of $\widetilde{v}_{k}$ converges to a holomorphic map $\widetilde{v}_{\infty}: \mathbb{C} \rightarrow \mathbb{C}^{n}$ that satisfies

$$
\int_{\mathbb{C}} \widetilde{v}_{\infty}^{*} \Theta^{*} d \lambda=0, \quad E\left(\widetilde{v}_{\infty}\right)<\infty
$$

and

$$
\left|d \widetilde{v}_{\infty}(0)\right|=1, \quad\left|d \widetilde{v}_{\infty}(u)\right| \leq 2 .
$$


But (13.18) implies $\widetilde{v}_{\infty}$ must be constant while (13.19) implies it cannot, a contradiction. This finishes the proof of Proposition 13.3.

By the elliptic regularity, we derive from (13.14) and Proposition 13.3 that the $C^{k}$ norm of $\bar{\xi}_{i}$ for all $k \geq 0$ is uniformly bounded on any bounded subset of $\mathbb{R} \times S^{1}$. Therefore, by Ascoli-Arzela's theorem, we can find a subsequence of $\bar{\xi}_{i}$ that converges to a holomorphic map

$$
\xi_{\infty}: \mathbb{R} \times S^{1} \rightarrow \mathbb{C}^{n}
$$

in compact $C^{\infty}$ topology. By (13.14), $\xi_{\infty}$ cannot be a constant map.

The following energy bound is an immediate consequences of Lemma 13.6.

Lemma 13.11. $E_{\text {int }}\left(\xi_{\infty}\right)$ and $E_{\text {neck }}\left(\xi_{\infty}\right)$ are finite.

Next we prove the following theorem.

Theorem 13.3. There exists a sequence of vectors $v_{i}$ and a subsequence of $\bar{\xi}_{i}=\xi_{i}-v_{i}$ that converges to a holomorphic map

$$
\xi_{\infty}: \mathbb{R} \times S^{1} \rightarrow \mathbb{C}^{n}
$$

in compact $C^{\infty}$-topology satisfying the following properties:

(1) $E_{\text {int }}\left(\xi_{\infty}\right)$ and $E_{\text {neck }}\left(\xi_{\infty}\right)$ are finite.

(2) In the decomposition $\xi_{\infty}=\left(s \circ \xi_{\infty}, \Theta \circ \xi_{\infty}\right)$ outside $B^{2 n}(1)$, we have

$$
\begin{aligned}
\lim _{\tau^{\prime} \rightarrow \infty} \Theta \circ \xi\left(\tau^{\prime}, t\right) & =\gamma_{+}(t), \\
\lim _{\tau^{\prime} \rightarrow-\infty} \Theta \circ \xi\left(\tau^{\prime}, t\right) & =\gamma_{-}(t),
\end{aligned}
$$

where $\gamma_{ \pm}$are the Reeb orbits of $S^{2 n-1}(1) \subset \mathbb{C}^{n} \cong\left(T_{p} M, \omega_{p}, J_{p}\right)$ associated to the tangent cones of $u_{+}, u_{-}$at the node $p=u_{+}(\infty)=$ $u_{-}(-\infty)$, respectively.

Proof. We start with the following result proved by Hofer $[\mathbf{H o}]$.

Lemma 13.12 (Theorem 31, [Ho]). Suppose that $\xi_{\infty}$ is a proper nonconstant pseudo-holomorphic with finite $E_{\Sigma}$-energy. There exists a closed Reeb orbit $\gamma: S^{1} \rightarrow S^{2 n-1}$ and a sequence $\tau_{k} \rightarrow \infty$ such that $\gamma_{k}=\xi_{\infty}\left(\tau_{k}, \cdot\right)$ converges in $C^{\infty}$ to $\gamma$. Similar statement holds also for $\tau_{k} \rightarrow \infty$.

We will now improve this convergence to

Proposition 13.4. $\xi_{\infty}$ is a proper holomorphic cylinder such that

$$
\lim _{\tau \rightarrow \pm \infty} \xi_{\infty}(\tau, \cdot)=\gamma_{ \pm}
$$

in $C^{\infty}$ where $\gamma_{ \pm}$are the Reeb orbits associated to the tangent cones of the node of $\left(u_{-}, u_{+}\right)$. 
Proof. The main tool for such a convergence result is Theorem 13.2 the characterization of the asymptotics of $J_{0}$-holomorphic maps with small $d \lambda$ energy $E_{d \lambda}$.

Let $\gamma_{a}$ be the Reeb orbit provided in Theorem 13.2 for $u=\xi_{\infty}$. We will treat only the case as $\tau \rightarrow+\infty$ since the case $\tau \rightarrow-\infty$ will be the same. In our situation, we have the vector $a=a \pm=\frac{d u_{ \pm}\left(o_{ \pm}\right)}{\left|d u_{ \pm}\left(o_{ \pm}\right)\right|}$.

We will show that there exists a constant $s_{1} \in \mathbb{R}$ such that $\xi_{\infty}$ satisfies

$$
\left|\xi_{\infty}(z)-u_{a, s_{1}}^{\mathrm{flat}}(z)\right|_{C^{k}} \rightarrow 0
$$

in exponential order as $|z| \rightarrow \infty$.

Let $E_{0}=E_{\text {neck }}\left(\xi_{\infty}\right)$. We take $e_{0}$ as in Theorem 13.2. Since $E\left(\xi_{\infty}\right)<\infty$, we can choose $S$ such that

$$
E\left(\xi_{\infty} ; S\right) \leq e_{0}
$$

Then, we can apply Theorem 13.2 to the restriction of $\xi_{\infty}$ to $[S, S+2 R] \times$ $[0,1]$.

Note $\xi_{\infty}([S, S+2 R] \times[0,1]) \subset[\log 4, \infty) \times S^{2 n-1}$. Put

$$
\gamma(t)=\xi_{\infty}(S+R, t), \quad \gamma_{i}(t)=\xi_{i}(S+R, t) .
$$

Then, by Lemma 13.3, we have:

$$
\int_{0}^{1} \gamma^{*} \lambda=\lim _{i \rightarrow \infty} \int_{0}^{1} \gamma_{i}^{*} \lambda \leq 3 \pi
$$

Therefore, we have constants $R_{2, j}$ and $s_{1, j}$ such that $R_{2, j} \rightarrow \infty$ and

$$
\left|\nabla^{k}\left(\xi_{\infty}-u_{a_{j}, s_{1, j}}^{\mathrm{flat}}\right)\right|(\tau, t) \leq C_{k} \mathrm{e}^{-c_{k}\left|\tau-S-R_{2, j}\right|}
$$

on $(\tau, t) \in\left[S+10, S-10+2 R_{2, j}\right] \times S^{1}$.

Since the intervals $\left[S+10, S-10+2 R_{2, j}\right]$ are nested as $R_{2, j} \nearrow \infty$, we should also have $s_{1, j} \rightarrow s_{1}$ as $j \rightarrow \infty$ for $s_{1}$ appearing in Theorem 13.2. Then (13.20) implies

$$
\left|\nabla^{k}\left(\xi_{\infty}-u_{a_{\infty}, s_{1}}^{\mathrm{flat}}\right)\right|(\tau, t) \leq C_{k}^{\prime} \mathrm{e}^{-c_{k}^{\prime}|\tau|}
$$

on $(\tau, t) \in[S+10, \infty) \times[0,1]$. Therefore, we have finished the proof.

Theorem 13.3 follows from Proposition 13.4.

Since every Reeb orbits of $S^{2 n-1}$ with the action $\int \gamma^{*} \lambda \leq 3 \pi$ is one of $\gamma_{a}$, we have $\gamma_{ \pm}=\gamma_{a_{ \pm}}$for some $a_{ \pm} \in S^{2 n-1}$. To finish the proof of Theorem 12.1, it remains to prove the $\varepsilon$-controlled convergence (12.10).

We take an isomorphism $\psi: \mathbb{R} \times S^{1} \rightarrow \mathbb{R} \times S^{1}$ such that $\psi( \pm \infty)= \pm \infty$ and

$$
d_{g_{\mathbb{C} n}^{\prime}}\left(\xi_{\infty} \circ \psi(0,0), 0\right)=\min _{(\tau, t) \in \mathbb{R} \times S^{1}} d_{g_{\mathbb{C}^{n}}^{\prime}}\left(\xi_{\infty}(\tau, t), 0\right) .
$$

We now define the map

$$
\psi_{i, \text { int }}:\left[-\infty, R_{i}\right) \times S^{1} \rightarrow \mathbb{H}
$$


for some $R_{i}$ to be determined later in the proof. Since we have

$$
\frac{1}{\varepsilon_{i}}\left(\left(\exp _{p}^{I}\right)^{-1} \circ u_{i} \circ \psi_{i, \text { int }}\right)+\tau^{\prime} a=\bar{\xi}_{i} \circ \psi_{i, \text { int }}=g_{v_{i}, \lambda_{i}} \circ \xi_{i} \circ \psi_{i, \text { int }}
$$

by the definitions of $g_{v_{i}, \lambda_{i}}$ and $\xi_{i}$ and $g_{v_{i}, \lambda_{i}} \xi_{i} \circ \psi_{i \text {,int }}$ converges to $\xi_{\infty}$, it

follows that $g_{v_{i}, \lambda_{i}}^{-1}\left(\frac{1}{\varepsilon_{i}}\left(\left(\exp _{p}^{I}\right)^{-1} \circ u_{i} \circ \psi_{i, \text { int }}\right)-\tau^{\prime} a\right)$ converges to $u_{a}^{\text {flat }, s_{1}}$ on compact $C^{\infty}$ topology. For the notational convenience, we will $\operatorname{drop}\left(\exp _{p}^{I}\right)^{-1}$ from $\left(\exp _{p}^{I}\right)^{-1} \circ u_{i} \circ \psi_{i \text {,int }}$ and just denote it by $u_{i} \circ \psi_{i \text {,int }}$.

By the diagonal sequence argument, we can choose a sequence $R_{i} \rightarrow \infty$ so that

$$
\lim _{i \rightarrow \infty} \sup _{R_{0} \leq \tau^{\prime} \leq 2 R_{i}}\left|\nabla^{k}\left(g_{v_{i}, \lambda_{i}}^{-1}\left(\frac{1}{\varepsilon_{1, i}} u_{i} \circ \psi_{i, \text { int }}\left(\tau^{\prime}, t^{\prime}\right)+\tau^{\prime} a\right)-u_{a, s_{1}}^{\text {flat }}\right)\right|=0 .
$$

It follows that there exist $S_{3}, I_{0}$ such that the following holds for $i \geq I_{0}$ :

$$
\int_{\left[S_{3}, 2 R_{i}\right) \times[0,1]}\left(\frac{1}{\varepsilon_{1, i}}\left(g_{v_{i}, \lambda_{i}}^{-1} \circ u_{i} \circ \psi_{i, \text { int }}\right)\right)^{*} d \lambda<e_{0},
$$

(2) $2 R_{i}-S_{3} \geq R_{0}$.

We can apply Theorem 13.2 to obtain $s_{i}^{\prime}$ such that

$$
\begin{aligned}
& \mid \nabla^{k}\left(g_{v_{i}, \lambda_{i}}^{-1}\left(\frac{1}{\varepsilon_{i}}\left(u_{i} \circ \psi_{i, \text { int }}\left(\tau^{\prime}, t^{\prime}\right)+\tau^{\prime} a\right)-u_{a_{i}^{\prime}, s_{i}^{\prime}}^{\mathrm{flat}}\left(\tau^{\prime}, t^{\prime}\right)\right) \mid\right. \\
& \quad \leq C_{k} \mathrm{e}^{-c_{k} \min \left\{\left|2 R_{i}-\tau\right|,\left|\tau-S_{3}\right|\right\}} .
\end{aligned}
$$

Comparing (13.21) with (13.22) we have $s_{i}^{\prime} \rightarrow s_{1}$. Perturbing $\psi_{i \text {,int }}$ slightly and re-choosing $s_{i}$, we may assume $s_{i}^{\prime}=0$.

Therefore, we obtain

$$
\begin{aligned}
& \mid \nabla^{k}\left(g_{v_{i}, \lambda_{i}}^{-1}\left(\frac{1}{\varepsilon_{i}}\left(u_{i} \circ \psi_{i, \text { int }}\left(\tau^{\prime}, t^{\prime}\right)+\tau^{\prime} a\right)-u_{a_{i}^{\prime}, s_{i}^{\prime}}^{\mathrm{flat}}\left(\tau^{\prime}, t^{\prime}\right)\right) \mid\right. \\
& \quad \leq C_{k} \mathrm{e}^{-c_{k} \min \left\{\left|2 R_{i}-\tau\right|,\left|\tau-S_{3}\right|\right\}} .
\end{aligned}
$$

Now the proof of Theorem 12.1 is finished.

\section{Part III. Application: a proof of PSS isomorphism}

In this part, we combine the analysis carried out in the previous sections with the standard cobordism argument to give the proof of $\Psi \circ \Phi=$ id in homology. For completeness's sake, we also give an explanation of the proof $\Phi \circ \Psi=\mathrm{i} d$ whose proof can be given by a more or less standard argument in Floer theory. The isomorphism proof in this part is complete as it is for the semi-positive $(M, \omega)$. However, we have been careful to provide our compactification of the relevant moduli spaces so that one can easily put 
Kuranishi structure [FOn] on them to generalize the isomorphism property to arbitrary compact $(M, \omega)$. Since this is not our main purpose of the paper, we do not pursue complete details and leave them for interested readers.

Remark 13.1. For example, observing that Proposition 5.2 holds for a generic choice of almost complex structures on any symplectic manifold, whether it is semi-positive or not, one can repeat the construction carried out in $[\mathbf{L u}]$ in our setting instead of in the setting of $[\mathbf{P S S}]$ that $[\mathbf{L u}]$ uses.

\section{Review of Floer complex and operators}

In this section, we give a brief summary of basic operators in the standard Floer homology theory. Details of construction of these operators are important for the argument in our proof of isomorphism property of the PSS map. While these constructions are standard, we closely follow the exposition presented in $[\mathrm{Oh2}, \mathrm{Oh} 4]$.

For each nondegenerate $H: S^{1} \times M \rightarrow \mathbb{R}$ with $\phi_{H}^{1}=\phi$, we know that the cardinality of $\operatorname{Per}(H)$ is finite. We consider the free $\mathbb{Q}$ vector space generated by the critical set of $\mathcal{A}_{H}$

$$
\operatorname{Crit} \mathcal{A}_{H}=\left\{[z, w] \in \widetilde{\Omega}_{0}(M) \mid z \in \operatorname{Per}(H)\right\} .
$$

Definition 14.1. Consider the formal sum

$$
\beta=\sum_{[z, w] \in \operatorname{Crit} \mathcal{A}_{H}} a_{[z, w]}[z, w], a_{[z, w]} \in \mathbb{Q} .
$$

(1) We call those $[z, w]$ with $a_{[z, w]} \neq 0$ generators of the sum $\beta$ and write

$$
[z, w] \in \beta .
$$

We also say that $[z, w]$ contributes to $\beta$ in that case.

(2) We define the support of $\beta$ by

$$
\operatorname{supp}(\beta):=\left\{[z, w] \in \operatorname{Crit} \mathcal{A}_{H} \mid a_{[z, w]} \neq 0 \text { in the sum (14.1) }\right\} .
$$

(3) We call the formal sum $\beta$ a Novikov Floer chain (or simply a Floer chain) if

$$
\#\left(\operatorname{supp}(\beta) \cap\left\{[z, w] \mid \mathcal{A}_{H}([z, w]) \geq \lambda\right\}\right)<\infty
$$

for any $\lambda \in \mathbb{R}$. We denote by $C F_{*}(H)$ the set of Floer chains.

We now explain the description of $C F(H)$ as a module over the Novikov ring as in $[\mathbf{F l 3}, \mathbf{H S}]$. Consider the abelian group

$$
\Gamma=\frac{\pi_{2}(M)}{\operatorname{ker} c_{1} \cap \operatorname{ker} \omega}
$$

and the formal sum

$$
R=\sum_{A \in \Gamma} r_{A} q^{A}, \quad r_{A} \in \mathbb{Q} .
$$


We define

$$
\operatorname{supp}(R)=\left\{A \in \Gamma \mid r_{A} \neq 0\right\} .
$$

The (upward) Novikov ring defined by

$$
\Lambda_{\omega}=\Lambda_{\omega}^{\uparrow}=\left\{\sum_{A \in \Gamma} r_{A} q^{A} \mid \forall \lambda \in \mathbb{R}, \#\left\{A \in \Gamma \mid r_{A} \neq 0, \omega(A)<\lambda\right\}<\infty\right\} .
$$

Then we have the valuation on $\Lambda_{\omega}$ given by

$$
v(R)=\min \{\omega(A) \mid A \in \operatorname{supp} R\} .
$$

We recall that $\Gamma$ acts on $\operatorname{Crit} \mathcal{A}_{H}$ by "gluing a sphere"

$$
[z, w] \mapsto[z, w \#(-A)]
$$

which in turn induces the multiplication of $\Lambda_{\omega}$ on $C F(H)$ by the convolution product. This enables one to regard $C F(H)$ as a $\Lambda_{\omega}$-module. We will try to consistently denote by $C F(H)$ as a $\Lambda_{\omega}$-module, and by $C F_{*}(H)$ as a graded $\mathbb{Q}$ vector space.

Suppose $H$ is a nondegenerate one-periodic Hamiltonian function and $J$ a one-periodic family of compatible almost complex structures. We first recall Floer's construction of the Floer boundary map, and the transversality conditions needed to define the Floer homology $H F_{*}(H, J)$ of the pair.

The following definition is useful for the later discussion.

Definition 14.2. Let $z, z^{\prime} \in \operatorname{Per}(H)$. We denote by $\pi_{2}\left(z, z^{\prime}\right)$ the set of homotopy classes of smooth maps

$$
u:[0,1] \times S^{1} \rightarrow M
$$

relative to the boundary

$$
u(0, t)=z(t), \quad u(1, t)=z^{\prime}(t) .
$$

We denote by $[u] \in \pi_{2}\left(z, z^{\prime}\right)$ its homotopy class and by $C$ a general element in $\pi_{2}\left(z, z^{\prime}\right)$.

We define by $\pi_{2}(z)$ the set of relative homotopy classes of the maps

$$
w: D^{2} \rightarrow M ;\left.\quad w\right|_{\partial D^{2}}=z .
$$

We denote by $\pi_{2}(M)$ the second homotopy class of maps $u: S^{2} \rightarrow M$. It acts on $\pi_{2}(z)$ and $\pi_{2}\left(z, z^{\prime}\right)$ (modulo the action of $\pi_{1}(M)$ ) by the obvious operation of a "gluing a sphere". Since the action of $\pi_{1}(M)$ on $\pi_{2}(M)$ does not change $c_{1}$ or the symplectic area, the operation of "gluing a sphere" induces the deck transformation action of $\Gamma_{\omega}$ on $\widetilde{\Omega}_{0}(M) \rightarrow \Omega_{0}(M)$ and so induces the structure of a principal $\Gamma$ fiber bundle

$$
\operatorname{Crit} \mathcal{A}_{H} \rightarrow \operatorname{Per}(H)
$$

on $\operatorname{Crit} \mathcal{A}_{H}$. 
Furthermore, there is a natural map of $C \in \pi_{2}\left(z, z^{\prime}\right)$

$$
(\cdot) \# C: \pi_{2}(z) \rightarrow \pi_{2}\left(z^{\prime}\right)
$$

induced by the gluing map

$$
w \mapsto w \# u .
$$

More specifically we will define the map $w \# u: D^{2} \rightarrow M$ in the polar coordinates $(r, \theta)$ of $D^{2}$ by the formula

$$
w \# u:(r, \theta)= \begin{cases}w(2 r, \theta), & \text { for } 0 \leq r \leq \frac{1}{2}, \\ w(2 r-1, \theta), & \text { for } \frac{1}{2} \leq r \leq 1\end{cases}
$$

once and for all. There is also the natural gluing map

$$
\begin{gathered}
\pi_{2}\left(z_{0}, z_{1}\right) \times \pi_{2}\left(z_{1}, z_{2}\right) \rightarrow \pi_{2}\left(z_{0}, z_{2}\right), \\
\left(u_{1}, u_{2}\right) \mapsto u_{1} \# u_{2} .
\end{gathered}
$$

We also explicitly represent the map $u_{1} \# u_{2}:[0,1] \times S^{1} \rightarrow M$ in the standard way once and for all similarly to (14.4).

Definition 14.3. We define the relative Conley-Zehnder index of $C \in \pi_{2}$ $\left(z, z^{\prime}\right)$ by

$$
\mu_{H}\left(z, z^{\prime} ; C\right)=\mu_{H}([z, w])-\mu_{H}\left(\left[z^{\prime}, w \# C\right]\right)
$$

for a (and so any) representative $u:[0,1] \times S^{1} \times M$ of the class $C$. We will also write $\mu_{H}(C)$, when there is no danger of confusion on the boundary condition.

It is easy to see that this definition does not depend on the choice of bounding disc $w$ of $z$, and so the function

$$
\mu_{H}: \pi_{2}\left(z, z^{\prime}\right) \rightarrow \mathbb{Z}
$$

is well defined.

We now denote by

$$
\mathcal{M}\left(H, J ; z, z^{\prime} ; C\right)
$$

the set of finite-energy solutions of

$$
\frac{\partial u}{\partial \tau}+J\left(\frac{\partial u}{\partial t}-X_{H}(u)\right)=0
$$

with the asymptotic condition and the homotopy condition

$$
u(-\infty)=z, \quad u(\infty)=z^{\prime} ; \quad[u]=C .
$$

(See $[\mathbf{F 1 3 , H S}]$.) Here we remark that although $u$ is a priori defined on $\mathbb{R} \times S^{1}$, it can be compactified into a continuous map $\bar{u}:[0,1] \times S^{1} \rightarrow M$ with the corresponding boundary condition

$$
\bar{u}(0)=z, \quad \bar{u}(1)=z^{\prime}
$$

due to the exponential decay property of finite energy solutions $u$ of (4.2), recalling we assume $H$ is nondegenerate. We will call $\bar{u}$ the compactified 
map of $u$. By some abuse of notation, we will also denote by $[u]$ the class $[\bar{u}] \in \pi_{2}\left(z, z^{\prime}\right)$ of the compactified map $\bar{u}$.

The Floer boundary map

$$
\partial_{(H, J)} ; C F_{k+1}(H) \rightarrow C F_{k}(H)
$$

is defined under the following conditions by studying equation (14.5) for a Floer-regular pair $(H, J)$ and satisfies $\partial \partial=0$, which enables us to take its homology. The Floer homology is defined by

$$
H F_{*}(H, J):=\operatorname{ker} \partial / \operatorname{im} \partial .
$$

One may regard this either as a graded $\mathbb{Q}$-vector space or as a $\Lambda_{\omega}$-module.

Next we describe the Floer chain map. When we are given a family $(\mathcal{H}, j)$ with $\mathcal{H}=\left\{H^{s}\right\}_{0 \leq s \leq 1}$ and $j=\left\{J^{s}\right\}_{0 \leq s \leq 1}$ and a cut-off function $\rho: \mathbb{R} \rightarrow$ $[0,1]$, the chain homomorphism

$$
h_{\mathcal{H}}=h_{(\mathcal{H}, j)}: C F_{*}\left(H_{\alpha}\right) \rightarrow C F_{*}\left(H_{\beta}\right)
$$

is defined by considering the nonautonomous form of (14.5).

Consider the pair $\left(\mathcal{H}_{\mathbb{R}}, j_{\mathbb{R}}\right)$ that are asymptotically constant, i.e., there exists $R>0$ such that

$$
J(\tau) \equiv J(\infty), \quad H(\tau) \equiv H(\infty)
$$

for all $\tau$ with $|\tau| \geq R$. We will always consider the form

$$
\left(\mathcal{H}_{\mathbb{R}}, j_{\mathbb{R}}\right)=\left\{\left(H^{\rho(\tau)}, J^{\rho(\tau)}\right)\right\}
$$

where $\left(H^{s}, J^{s}\right)$ is a homotopy over $s \in[0,1]$ and $\rho: \mathbb{R} \rightarrow[0,1]$ is a function as defined before. We study the following equation (14.5):

$$
\frac{\partial u}{\partial \tau}+J^{\rho(\tau)}\left(\frac{\partial u}{\partial t}-X_{H^{\rho(\tau)}}(u)\right)=0
$$

We denote by

$$
\mathcal{M}(\mathcal{H}, j ; \rho)
$$

the set of finite-energy solutions of (14.7).

For a Floer-regular pair $(\mathcal{H}, j)$, we can define a continuous map of degree zero

$$
h_{(\mathcal{H}, j ; \rho)}: C F\left(H_{\alpha}\right) \rightarrow C F\left(H_{\beta}\right)
$$

by the matrix element $n_{(\mathcal{H}, j ; \rho)}\left(\left[z_{\alpha}, w_{\alpha}\right],\left[z_{\beta}, w_{\beta}\right]\right)$ similarly as for the boundary map. Then $h_{(\mathcal{H}, j)}$ has degree 0 and satisfies the identity

$$
h_{(\mathcal{H}, j ; \rho)} \circ \partial_{\left(H_{\alpha}, J_{\alpha}\right)}=\partial_{\left(H_{\beta}, J_{\beta}\right)} \circ h_{(\mathcal{H}, j ; \rho)} .
$$

Two such chain maps $h_{\left(j^{1}, \mathcal{H}^{1}\right)}, h_{\left(j^{2}, \mathcal{H}^{2}\right)}$ are also chain homotopic [Fl3].

Now we examine Floer chain homotopy maps and the composition law

$$
h_{\alpha \gamma}=h_{\beta \gamma} \circ h_{\alpha \beta}
$$


of the Floer isomorphism

$$
h_{\alpha \beta}: H F_{*}\left(H_{\alpha}\right) \rightarrow H F_{*}\left(H_{\beta}\right) .
$$

Although the above isomorphism in homology depends only on the end Hamiltonians $H_{\alpha}$ and $H_{\beta}$, the corresponding chain map depends on the homotopy $\mathcal{H}=\{H(\eta)\}_{0 \leq \eta \leq 1}$ between $H_{\alpha}$ and $H_{\beta}$, and also on the homotopy $j=\{J(\eta)\}_{0 \leq \eta \leq 1}$. Let us fix nondegenerate Hamiltonians $H_{\alpha}, H_{\beta}$ and a homotopy $\mathcal{H}$ between them. We then fix a homotopy $j=\{J(\eta)\}_{0 \leq \eta \leq 1}$ of compatible almost complex structures and a cut-off function $\rho: \mathbb{R} \rightarrow[0,1]$.

We recall that we have imposed the homotopy condition

$$
\left[w^{+}\right]=\left[w^{-} \# u\right] ; \quad[u]=C \quad \text { in } \quad \pi_{2}\left(z^{-}, z^{+}\right)
$$

in the definition of $\mathcal{M}\left(H, J ;\left[z^{-}, w^{-}\right],\left[z^{+}, w^{+}\right]\right)$and of $\mathcal{M}\left((\mathcal{H}, j ; \rho) ;\left[z_{\alpha}, w_{\alpha}\right]\right.$, $\left.\left[z_{\beta}, w_{\beta}\right]\right)$. One consequence of $(14.9)$ is

$$
\left[z^{+}, w^{+}\right]=\left[z^{+}, w^{-} \# u\right], \quad \text { in } \quad \Gamma
$$

but the latter is a weaker condition than the former. In other words, there could be more than one distinct elements $C_{1}, C_{2} \in \pi_{2}\left(z^{-}, z^{+}\right)$such that

$$
\mu\left(z^{-}, z^{+} ; C_{1}\right)=\mu\left(z^{-}, z^{+} ; C_{2}\right), \omega\left(C_{1}\right)=\omega\left(C_{2}\right) .
$$

When we are given a homotopy $(\bar{j}, \overline{\mathcal{H}})$ of homotopies with $\bar{j}=\left\{j_{\kappa}\right\}, \overline{\mathcal{H}}=$ $\left\{\mathcal{H}_{\kappa}\right\}$, we also define the elongations $\mathcal{H}^{\bar{\rho}}$ of $\mathcal{H}_{\kappa}$ by a homotopy of cut-off functions $\bar{\rho}=\left\{\rho_{\kappa}\right\}$ : we have

$$
\mathcal{H}^{\bar{\rho}}=\left\{\mathcal{H}_{\kappa}^{\rho_{\kappa}}\right\}_{0 \leq \kappa \leq 1}
$$

Consideration of the parameterized version of (14.7) for $0 \leq \kappa \leq 1$ defines the chain homotopy map

$$
H_{\overline{\mathcal{H}}}: C F_{*}\left(H_{\alpha}\right) \rightarrow C F_{*}\left(H_{\beta}\right),
$$

which has degree +1 and satisfies

$$
h_{\left(j_{1}, \mathcal{H}_{1} ; \rho_{1}\right)}-h_{\left(j_{0}, \mathcal{H}_{0}: \rho_{0}\right)}=\partial_{\left(J^{1}, H^{1}\right)} \circ H_{\overline{\mathcal{H}}}+H_{\overline{\mathcal{H}}} \circ \partial_{\left(J^{0}, H^{0}\right)} .
$$

Again the map $H_{\overline{\mathcal{H}}}$ depends on the choice of a homotopy $\bar{j}$ and $\bar{\rho}=$ $\left\{\rho_{\kappa}\right\}_{0 \leq \kappa \leq 1}$ connecting the two functions $\rho_{0}, \rho_{1}$. Therefore, we will denote

$$
H_{\overline{\mathcal{H}}}=H_{(\overline{\mathcal{H}}, \bar{j} ; \bar{\rho})}
$$

as well. Equation (14.10) in particular proves that two chain maps for different homotopies $\left(j_{0}, \mathcal{H}_{0} ; \rho_{0}\right)$ and $\left(j_{1}, \mathcal{H}_{1} ; \rho_{1}\right)$ connecting the same end points are chain homotopic and so proves that the isomorphism (14.8) in homology is independent of the homotopies $(\overline{\mathcal{H}}, \bar{j})$ or of $\bar{\rho}$.

Next, we consider the triple

$$
\left(H_{\alpha}, H_{\beta}, H_{\gamma}\right)
$$


of Hamiltonians and homotopies $\mathcal{H}_{1}, \mathcal{H}_{2}$ connecting from $H_{\alpha}$ to $H_{\beta}$ and $H_{\beta}$ to $H_{\gamma}$, respectively. We define their concatenation $\mathcal{H}_{1} \# \mathcal{H}_{2}=\left\{H_{3}(s)\right\}_{1 \leq s \leq 1}$ by

$$
H_{3}(s)= \begin{cases}H_{1}(2 s), & 0 \leq s \leq \frac{1}{2} \\ H_{2}(2 s-1), & \frac{1}{2} \leq s \leq 1\end{cases}
$$

We note that due to the choice of the cut-off function $\rho$, the continuity equation (14.7) is autonomous for the region $|\tau|>R$, i.e., is invariant under the translation by $\tau$. When we are given a triple $\left(H_{\alpha}, H_{\beta}, H_{\gamma}\right)$, this fact enables us to glue solutions of two such equations corresponding to the pairs $\left(H_{\alpha}, H_{\beta}\right)$ and $\left(H_{\beta}, H_{\gamma}\right)$, respectively.

Now a more precise explanation is in order. For a given pair of cut-off functions

$$
\rho=\left(\rho_{1}, \rho_{2}\right)
$$

and a positive number $R>0$, we define an elongated homotopy of $\mathcal{H}_{1} \# \mathcal{H}_{2}$

$$
\mathcal{H}_{1} \#(\rho ; R) \mathcal{H}_{2}=\left\{H_{(\rho ; R)}(\tau)\right\}_{-\infty<\tau<\infty}
$$

by

$$
H_{(\rho ; R)}(\tau, t, x)= \begin{cases}H_{1}\left(\rho_{1}(\tau+2 R), t, x\right), & \tau \leq 0 \\ H_{2}\left(\rho_{2}(\tau-2 R), t, x\right), & \tau \geq 0\end{cases}
$$

Note that

$$
H_{(\rho ; R)} \equiv \begin{cases}H_{\alpha}, & \text { for } \tau \leq-\left(R_{1}+2 R\right) \\ H_{\beta}, & \text { for }-R \leq \tau \leq R \\ H_{\gamma}, & \text { for } \tau \geq R_{2}+2 R\end{cases}
$$

for some sufficiently large $R_{1}, R_{2}>0$ depending on the cut-off functions $\rho_{1}, \rho_{2}$ and the homotopies $\mathcal{H}_{1}, \mathcal{H}_{2}$, respectively. In particular this elongated homotopy is always smooth, even when the usual glued homotopy $\mathcal{H}_{1} \# \mathcal{H}_{2}$ may not be so. We define the elongated homotopy $j_{1} \#(\rho ; R) j_{2}$ of $j_{1} \# j_{2}$ in a similar way.

For an elongated homotopy $\left(j_{1} \#(\rho ; R) j_{2}, \mathcal{H}_{1} \#(\rho, R) \mathcal{H}_{2}\right)$, we consider the associated perturbed Cauchy-Riemann equation

$$
\left\{\begin{array}{l}
\frac{\partial u}{\partial \tau}+J_{3}^{\rho(\tau)}\left(\frac{\partial u}{\partial t}-X_{H_{3}^{\rho(\tau)}}(u)\right)=0, \\
\lim _{\tau \rightarrow-\infty} u(\tau)=z^{-}, \lim _{\tau \rightarrow \infty} u(\tau)=z^{+}
\end{array}\right.
$$

with the condition (14.9).

Now let $u_{1}$ and $u_{2}$ be given solutions of (14.7) associated to $\rho_{1}$ and $\rho_{2}$ respectively. If we define the pre-gluing map $u_{1} \#_{R} u_{2}$ by the formula

$$
u_{1} \#_{R} u_{2}(\tau, t)= \begin{cases}u_{1}(\tau+2 R, t), & \text { for } \tau \leq-R, \\ u_{2}(\tau-2 R, t), & \text { for } \tau \geq R\end{cases}
$$


and a suitable interpolation between them by a partition of unity on the region $-R \leq \tau \leq R$, the assignment defines a diffeomorphism

$$
\left(u_{1}, u_{2}, R\right) \rightarrow u_{1} \#{ }_{R} u_{2}
$$

from

$$
\mathcal{M}\left(j_{1}, \mathcal{H}_{1} ;\left[z_{1}, w_{1}\right],\left[z_{2}, w_{2}\right]\right) \times \mathcal{M}\left(j_{2}, \mathcal{H}_{2} ;\left[z_{2}, w_{2}\right],\left[z_{3}, w_{3}\right]\right) \times\left(R_{0}, \infty\right)
$$

onto its image, provided $R_{0}$ is sufficiently large. Denote by $\bar{\partial}_{(\mathcal{H}, j ; \rho)}$ the corresponding perturbed Cauchy-Riemann operator

$$
u \mapsto \frac{\partial u}{\partial \tau}+J_{3}^{\rho(\tau)}\left(\frac{\partial u}{\partial t}-X_{H_{3}^{\rho(\tau)}}(u)\right)
$$

acting on the maps $u$ satisfying the asymptotic condition $u( \pm \infty)=z^{ \pm}$and fixed homotopy condition $[u]=C \in \pi_{2}\left(z^{-}, z^{+}\right)$. By perturbing $u_{1} \#_{R} u_{2}$ by the amount that is smaller than the error for $u_{1} \#_{R} u_{2}$ to be a genuine solution, i.e., less than a weighted $L^{p}$-norm, for $p>2$,

$$
\left\|\bar{\partial}_{(\mathcal{H}, j ; \rho)}\left(u_{1} \#(\rho ; R) u_{2}\right)\right\|_{p}
$$

in a suitable $W^{1, p}$ space of $u$ 's, one can construct a unique genuine solution near $u_{1} \#_{R} u_{2}$. By an abuse of notation, we will denote this genuine solution also by $u_{1} \#_{R} u_{2}$. Then the corresponding map defines an embedding

$$
\begin{aligned}
& \mathcal{M}\left(j_{1}, \mathcal{H}_{1} ;\left[z_{1}, w_{1}\right],\left[z_{2}, w_{2}\right]\right) \times \mathcal{M}\left(j_{2}, \mathcal{H}_{2} ;\left[z_{2}, w_{2}\right],\left[z_{3}, w_{3}\right]\right) \times\left(R_{0}, \infty\right) \\
& \quad \rightarrow \mathcal{M}\left(j_{1} \#(\rho ; R) j_{2}, \mathcal{H}_{1} \#(\rho ; R) \mathcal{H}_{2} ;\left[z_{1}, w_{1}\right],\left[z_{3}, w_{3}\right]\right) .
\end{aligned}
$$

Especially, when we have

$$
\mu_{H_{\beta}}\left(\left[z_{2}, w_{2}\right]\right)-\mu_{H_{\alpha}}\left(\left[z_{1}, w_{1}\right]\right)=\mu_{H_{\gamma}}\left(\left[z_{3}, w_{3}\right]\right)-\mu_{H_{\beta}}\left(\left[z_{2}, w_{2}\right]\right)=0
$$

both $\mathcal{M}\left(j_{1}, \mathcal{H}_{1} ;\left[z_{1}, w_{1}\right],\left[z_{2}, w_{2}\right]\right)$ and $\mathcal{M}\left(j_{2}, \mathcal{H}_{2} ;\left[z_{2}, w_{2}\right],\left[z_{3}, w_{3}\right]\right)$ are compact, and so consist of a finite number of points. Furthermore, the image of the above mentioned embedding exhausts the "end" of the moduli space

$$
\mathcal{M}\left(j_{1} \#(\rho ; R) j_{2}, \mathcal{H}_{1} \#(\rho ; R) \mathcal{H}_{2} ;\left[z_{1}, w_{1}\right],\left[z_{3}, w_{3}\right]\right)
$$

and the boundary of its compactification consists of the broken trajectories

$$
u_{1} \#(\rho ; \infty) u_{2}=u_{1} \# \infty u_{2} .
$$

This then proves the following gluing identity

Proposition 14.1. There exists $R_{0}>0$ such that for any $R \geq R_{0}$ we have

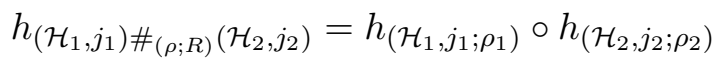

as a chain map from $C F_{*}\left(H_{\alpha}\right)$ to $C F_{*}\left(H_{\gamma}\right)$. 
Here we remind the readers that the homotopy $\mathcal{H}_{1} \#(\rho ; R) \mathcal{H}_{2}$ itself is an elongated homotopy of the glued homotopy $\mathcal{H}_{1} \# \mathcal{H}_{2}$. This proposition then gives rise to the composition law $h_{\alpha \gamma}=h_{\beta \gamma} \circ h_{\alpha \beta}$ in homology.

This finishes the summary of construction of Floer complex and basic operations in Floer theory. In particular, the chain homotopy map is defined whenever the family $(\overline{\mathcal{H}}, \bar{J})$ where $\overline{\mathcal{H}}=\left\{\mathcal{H}_{\kappa}\right\}, \bar{J}=\left\{J^{\kappa}\right\}$ are smooth families over $0 \leq \kappa \leq 1$. However, the chain homotopy map used in PSS map that we have been considering in the present paper is not of this kind but induced by the concatenation of two noncompact homotopies over $-\infty \leq \ell<0$ and $0<\varepsilon \leq 1$.

\section{5. $\Psi \circ \Phi=\mathrm{i} d$; Floer via Morse back to Floer}

Consider the PSS deformation defined over $\kappa \in[-\infty, 1]$. We fix a homotopy $\left(K^{\kappa}, J^{\kappa}\right)$ as any generic homotopy from $\left(K^{\varepsilon_{0}}, J^{\varepsilon_{0}}\right)$ to $\left(K^{1}, J^{1}\right)=$ $(H(t, x), J)$.

Fix a sufficiently small $\varepsilon_{0}>0$ and a sufficiently large $\ell_{0}>0$. We divide the deformation into the following five pieces

$$
\begin{gathered}
\left(K^{\kappa}, J^{\kappa}\right), \quad \text { for }\left[\varepsilon_{0} \leq \kappa \leq 1\right], \\
\left(K_{R(\varepsilon)}, J_{R(\varepsilon)}\right), \quad \text { for } 0<\kappa \leq \varepsilon_{0}, \\
\left(H^{\rho_{-}}, J^{\rho_{-}}\right)_{o_{-}} *\left(f, J_{0} ;[-\ell, \ell]\right) *_{o_{+}}\left(H^{\rho_{+}}, J^{\rho_{+}}\right), \quad \text { for }-\ell_{0} \leq \ell<0:
\end{gathered}
$$

and

$$
\left(H^{\rho_{-}}, J^{\rho_{-}}\right)_{o_{-}} *\left(f, J_{0} ;[-\ell, \ell]\right) *_{o_{+}}\left(H^{\rho_{+}}, J^{\rho_{+}}\right), \quad \text { for }-\infty \leq \ell<-\ell_{0}:
$$

Here $\left(f, J_{0} ;[-\ell, \ell]\right)$ stands for the deformation

$$
\ell \in(-\infty, 0) \mapsto\left(f, J_{0} ;[-\ell, \ell]\right),
$$

where $f$ is a Morse function with respect to the metric $g_{J_{0}}$ and we consider its gradient trajectories over the interval $[-\ell, \ell]$.

We denote by $\left.\mathcal{M}_{\kappa}^{\Psi \Phi}\left(\left[z_{-}, w_{-}\right]\right),\left[z_{+}, w_{+}\right]\right)$the moduli space of configuration corresponding to $\kappa$ and form the parameterized moduli space

$$
\overline{\mathcal{M}}_{\Psi \Phi}^{\mathrm{para}}\left(\left[z_{-}, w_{-}\right],\left[z_{+}, w_{+}\right] ; f\right)=\bigcup_{\kappa \in[-1, \infty]} \mathcal{M}_{\kappa}^{\Psi \Phi}\left(\left[z_{-}, w_{-}\right],\left[z_{+}, w_{+}\right]\right) .
$$

By the nondegeneracy hypothesis and the index condition, $\mathcal{M}_{\kappa}^{\Psi \Phi}$ is empty except at a finite number of points

$$
\kappa \in\left(-\ell_{0},-\ell_{1}\right) \cup\left(\varepsilon_{0}, 1\right)
$$

but a priori those $\kappa$ could be accumulated in $\left[-\ell_{1}, \varepsilon_{0}\right]$. The one-jet transversality of the enhanced nodal Floer trajectory moduli space, which corresponds to $\kappa=0$ and the main gluing result of the present paper, proves that this accumulation cannot be possible. As a result,

$$
\left.\mathcal{M}_{\kappa}^{\Psi \Phi}\left(\left[z_{-}, w_{-}\right]\right),\left[z_{+}, w_{+}\right]\right)=\emptyset
$$


for all $\kappa \in\left[-\ell_{1}, \varepsilon_{0}\right]$ if we choose $\ell_{1}, \varepsilon_{0}$ sufficiently small. Together with the main gluing compactness result of the present paper, this discussion proves the following proposition:

Proposition 15.1. There exist constants $\ell_{0}, \ell_{1}, \varepsilon_{0}$ and $\varepsilon_{1}$ such that the followings hold:

(1) Suppose $\mu_{H}\left(\left[z_{-}, w_{-}\right]\right)-\mu_{H}\left(\left[z_{+}, w_{+}\right]\right)=-1$. Then $\overline{\mathcal{M}}_{\Psi \Phi}^{\text {para }}\left(\left[z_{-}, w_{-}\right]\right)$, $\left.\left[z_{+}, w_{+}\right]\right)$is a compact zero-dimensional manifold such that

$$
\mathcal{M}_{\kappa}^{\Psi \Phi}\left(\left[z_{-}, w_{-}\right],\left[z_{+}, w_{+}\right]\right)=\emptyset
$$

for $\kappa \in\left[-\infty,-\ell_{0}\right] \cup\left[-\ell_{1}, \varepsilon_{0}\right] \cup\left[1-\varepsilon_{1}, 1\right]$.

(2) Suppose $\mu_{H}\left(\left[z_{-}, w_{-}\right]\right)-\mu_{H}\left(\left[z_{+}, w_{+}\right]\right)=0$. Then $\overline{\mathcal{M}}_{\Psi \Phi}^{\text {para }}\left(\left[z_{-}, w_{-}\right]\right)$, $\left.\left[z_{+}, w_{+}\right] ; f\right)$ is a compact one-dimensional manifold with boundary given by

$$
\begin{aligned}
\left.\partial \overline{\mathcal{M}}_{\Psi \Phi}^{\text {para }}\left(\left[z_{-}, w_{-}\right]\right),\left[z_{+}, w_{+}\right] ; f\right) & =\mathcal{M}_{1}\left(\left[z_{-}, w_{-}\right],\left[z_{+}, w_{+}\right]\right) \cup \mathcal{M}_{-\infty}\left(\left[z_{-}, w_{-}\right],\left[z_{+}, w_{+}\right]\right) \\
& \cup\left(\bigcup_{[z, w]} \overline{\mathcal{M}}_{\Psi \Phi}^{\text {para }}\left(\left[z_{-}, w_{-}\right],[z, w]\right) \# \mathcal{M}_{\kappa=1}\left([z, w],\left[z_{+}, w_{+}\right]\right)\right) \\
& \cup\left(\bigcup_{[z, w]} \mathcal{M}_{\kappa=1}\left(\left[z_{-}, w_{-}\right],[z, w]\right) \# \overline{\mathcal{M}}_{\Psi \Phi}^{\text {para }}\left([z, w],\left[z_{+}, w_{+}\right]\right)\right),
\end{aligned}
$$

where the union is taken over all $[z, w]$ with $\mu_{H}\left(\left[z_{-}, w_{-}\right]\right)-\mu_{H}$ $([z, w])=-1$ for the first and $\mu_{H}([z, w])-\mu_{H}\left(\left[z_{+}, w_{+}\right]\right)=-1$ for the second.

Statement (1) in this proposition allows one to define the matrix coefficients the order

$$
\left.\# \overline{\mathcal{M}}_{\Psi \Phi}^{\mathrm{para}}\left(\left[z_{-}, w_{-}\right]\right),\left[z_{+}, w_{+}\right] ; f\right) .
$$

We then define the map

$$
h_{\mathrm{pss}}^{\Psi \Phi}: C F_{*}(H) \rightarrow C F_{*+1}(H)
$$

by the matrix coefficients

$$
\left\langle h_{\mathrm{pss}}^{\Psi \Phi}\left(\left[z_{-}, w_{-}\right],\left[z_{+}, w_{+}\right]\right\rangle:=\# \overline{\mathcal{M}}_{\Psi \Phi}^{\mathrm{para}}\left(\left[z_{-}, w_{-}\right]\right),\left[z_{+}, w_{+}\right] ; f\right) .
$$

Then Statement (2) concerning the description of the boundary of the one dimensional moduli space $\left.\overline{\mathcal{M}}_{\Psi \Phi}^{\text {para }}\left(\left[z_{-}, w_{-}\right]\right),\left[z_{+}, w_{+}\right] ; f\right)$ is translated into the equation

$$
\Psi \circ \Phi-\mathrm{i} d=\partial \circ h_{\mathrm{pss}}^{\Psi \Phi}+h_{\mathrm{pss}}^{\Psi \Phi} \circ \partial .
$$

This finishes the proof $\Psi \circ \Phi=\mathrm{i} d$ in homology. 


\section{6. $\Phi \circ \Psi=\mathrm{i} d$; Morse via Floer back to Morse}

In this section, for each given pair $p, q \in$ Crit $f$, we consider the parameterized moduli space

$$
\overline{\mathcal{M}}_{\Phi \Psi}^{\mathrm{para}}(p, q)=\bigcup_{0 \leq R \leq \infty} \mathcal{M}_{R}^{\Phi \Psi}(p, q): .
$$

We define $\mathcal{M}_{R}^{\Phi \Psi}(p, q)$ in the following way.

First for each $0<R<\infty$, we introduce the moduli space $\mathcal{M}_{(2 ; 0,0))}\left(\left(K^{R}\right.\right.$, $\left.J^{R}\right)$ ) of finite energy solutions of

$$
\bar{\partial}_{\left(K^{R}, J^{R}\right)} u=0
$$

on $\Sigma$ which is a Riemann surface with two marked points $\left\{o_{-}, o_{+}\right\}$so that $\Sigma \backslash\left\{o_{ \pm}\right\} \cong \mathbb{R} \times S^{1}$ conformally. We first define a family of Riemann surface $\left(\Sigma, j_{R}\right)$ by the connected sum

$$
\left(D^{-}, o_{-}\right) \cup C_{R} \cup\left(D^{+}, o_{+}\right), \quad j_{R}=j_{D^{-}} \# j_{C_{R}} \# j_{D^{+}},
$$

where $C_{R}$ is the cylinder $[-R, R] \times S^{1}, j_{C_{R}}$ the standard conformal structure and $j_{R}$ is the obvious glued conformal structure on $D^{-} \cup C_{R} \cup D^{+}$. We denote $(\tau, t)$ the conformal coordinates on $D^{-} \cup C_{R} \cup D^{+} \backslash\left\{o_{-}, o_{+}\right\}$extending the standard coordinates on $\mathbb{C}_{R}$.

In this conformal coordinates, we fix a family of cut-off functions $\chi^{R}$ by

$$
\chi^{R}(\tau)= \begin{cases}1-\kappa^{+}(\tau-R), & \text { for } \tau \geq 0, \\ 1-\kappa^{-}(\tau+R), & \text { for } \tau \leq 0,\end{cases}
$$

for $1 \leq R<\infty$, and $\chi^{R}=R \chi^{1}$ for $0 \leq R \leq 1$. We note that $\chi^{0} \equiv 0$ and $\chi^{R}$ has compact support and $\chi^{R} \equiv 1$ on any given compact subset if $R$ is sufficiently large. Therefore, equation (16.1) is reduced to $\bar{\partial}_{J_{0}} u=0$ near the marked points $o_{ \pm}$. Then we define $\left(K^{R}, J^{R}\right)$ as in Subsection 5.5.

We have two evaluations

$$
\mathrm{ev}_{o_{ \pm}}: \mathcal{M}_{(2 ; 0,0)}\left(K^{R}, J^{R}\right) \rightarrow M ; \quad \mathrm{ev}_{o_{ \pm}}(u)=u\left(o_{ \pm}\right) .
$$

We denote

$$
\begin{aligned}
& \widetilde{\mathcal{M}}^{-}(p ; f)=\{\chi: \mathbb{R} \times M \mid \dot{\chi}+\nabla f(\chi)=0, \chi(-\infty)=p\}, \\
& \widetilde{\mathcal{M}}^{+}(q ; f)=\{\chi: \mathbb{R} \times M \mid \dot{\chi}+\nabla f(\chi)=0, \chi(+\infty)=q\}
\end{aligned}
$$

and define

$$
\begin{aligned}
\widetilde{\mathcal{M}}_{1}^{-}(p ; f) & =\widetilde{\mathcal{M}}^{-}(p ; f) \times \mathbb{R}, \\
\widetilde{\mathcal{M}}_{1}^{+}(q ; f) & =\widetilde{\mathcal{M}}^{+}(q ; f) \times \mathbb{R} .
\end{aligned}
$$

$\tau_{0} \in \mathbb{R}$ acts on both by the action

$$
\left(\tau_{0},(\chi, \tau)\right) \mapsto\left(\chi\left(*-\tau_{0}\right), \tau+\tau_{0}\right) .
$$


This action is free and so their quotients

$$
\mathcal{M}_{1}^{-}(p ; f)=\widetilde{\mathcal{M}}_{1}^{-}(p ; f) / \mathbb{R}, \quad \mathcal{M}_{1}^{+}(q ; f)=\widetilde{\mathcal{M}}_{1}^{+}(q ; f) / \mathbb{R}
$$

become smooth manifold of dimension $\mu_{\text {Morse }}(p ; f)$ and $2 n-\mu_{\text {Morse }}(q ; f)$, respectively. We have the evaluation maps

$$
e v_{+}: \mathcal{M}_{1}^{+}(q ; f) \rightarrow M, \quad e v_{-}: \mathcal{M}_{1}^{-}(p ; f) \rightarrow M,
$$

whose image has one-one correspondence with the unstable manifold $W^{u}(p ; f)$ and the stable manifold $W^{s}(q ; f)$, respectively.

Now we define the moduli space $\mathcal{M}_{R}^{\Phi \Psi}(p, q ; A)$ to be the fiber product

$$
\begin{aligned}
\mathcal{M}_{R}^{\Phi \Psi}(p, q ; A) & =\mathcal{M}_{1}^{-}(p ; f)_{e v_{-}} \times_{e v_{o_{-}}} \mathcal{M}_{(2 ; 0,0)}\left(K^{R}, J^{R} ; A\right)_{e v_{o_{+}}} \times_{e v_{+}} \mathcal{M}_{1}^{+}(q ; f) \\
& =\left\{\left(\left(\chi_{-}, \tau_{-}\right), u,\left(\chi_{+}, \tau_{+}\right)\right) \mid \chi_{-}\left(\tau_{-}\right)=u\left(o_{-}\right), \chi_{+}\left(\tau_{+}\right)=u\left(o_{+}\right)\right\}
\end{aligned}
$$

and

$$
\overline{\mathcal{M}}^{\Phi \Psi, \text { para }}(p, q ; A)=\bigcup_{0 \leq R \leq \infty} \mathcal{M}_{R}^{\Phi \Psi}(p, q ; A) .
$$

A straightforward calculation shows that

$$
\operatorname{dim}^{\text {virt }} \mathcal{M}_{R}^{\Phi \Psi}(p, q ; A)=\mu_{\text {Morse }}(p)-\mu_{\text {Morse }}(q)+2 c_{1}(A) .
$$

Proposition 16.1. Choose a generic pair $\left(f, J_{0}\right)$.

(1) Suppose that $\mu_{\text {Morse }}(p)-\mu_{\text {Morse }}(q)+2 c_{1}(A)=-1$. Then there exist some $\varepsilon_{1}>0$ and $R_{1}>0$ such that Then $\overline{\mathcal{M}}_{R}^{\Phi \Psi, \text { para }}(p, q ; A)$ is a compact 0-dimensional manifold such that

$$
\mathcal{M}_{R}^{\Phi \Psi}(p, q ; A)=\emptyset
$$

if $0 \leq R \leq \varepsilon_{1}$ or $R \geq R_{1}$.

(2) Suppose that $\mu_{\text {Morse }}(p)-\mu_{\text {Morse }}(q)+2 c_{1}(A)=0$. Then $\overline{\mathcal{M}}^{\Phi \Psi \text {,para }}$ $(p, q ; A)$ is a compact one-manifold with boundary given by

$$
\partial \overline{\mathcal{M}}^{\Phi \Psi, \text { para }}(p, q ; A)=\mathcal{M}_{0}^{\Phi \Psi}(p, q ; A) \cup \mathcal{M}_{\infty}^{\Phi \Psi}(p, q ; A) \cup \bigcup_{r} \overline{\mathcal{M}}^{\Phi \Psi}(p, r ; A)
$$

where the union $\bigcup_{r}$ is taken over $r \in$ Crit $f$ such that

$$
\mu_{\text {Morse }}(p)-\mu_{\text {Morse }}(r)+2 c_{1}(A)=-1 \text {. }
$$

Proof. We recall that when $R=0$, equation (16.1) is reduced to $\bar{\partial}_{J_{0}} u=0$. Since $\mu_{\text {Morse }}(p)-\mu_{\text {Morse }}(q)+2 c_{1}(A)=-1$ represents the virtual dimension of $\mathcal{M}_{0}^{\Phi \Psi}(p, q ; A), \mathcal{M}_{0}^{\Phi \Psi}(p, q ; A)$ must be empty for a generic choice of $\left(f, J_{0}\right)$. Here we emphasize the fact that this moduli space depends only on $\left(f, J_{0}\right)$ for which the genericity argument can be applied independent of the parameter $R$. Therefore, the same must be the case when $R_{1} \leq \varepsilon_{1}$ for a sufficiently small $\varepsilon_{1}>0$. This finishes the proof.

We leave the proof of Statement (2) to the readers. 
Using Statement (1), we define the chain homotopy map

$$
h_{\mathrm{pss}}^{\Phi \Psi}: C M_{*}\left(f, J_{0} ; \Lambda_{\omega}\right) \rightarrow C M_{*+1}\left(f, J_{0} ; \Lambda_{\omega}\right)
$$

by the matrix element

$$
\left\langle h_{\mathrm{pss}}^{\Phi \Psi}(p), q \#(-A)\right\rangle=\sum_{(r, A)} \#\left(\bigcup_{r} \overline{\mathcal{M}}^{\Phi \Psi, \mathrm{para}}(p, r ; A)\right) .
$$

Next, we prove the following lemma:

Lemma 16.1. Suppose $\mu_{\text {Morse }}(p)-\mu_{\text {Morse }}(q)+2 c_{1}(A)=0$. Then if $A \neq 0$,

$$
\operatorname{dim} \mathcal{M}_{0}^{\Phi \Psi}(p, q ; A) \geq 2
$$

unless $\mathcal{M}_{0}^{\Phi \Psi}(p, q ; A)=\emptyset$. And when $A=0$, we have

$$
\operatorname{dim} \mathcal{M}_{0}^{\Phi \Psi}(p, q ; A) \geq 1
$$

unless $p=q$.

Proof. If $A \neq 0, u$ is nonconstant in $\left(u ; o_{-}, o_{+}\right) \in \mathcal{M}_{(2 ; 0,0))}\left(\left(K^{R}, J^{R}\right)\right)$. Then the conformal automorphism on the domain $\left(\Sigma ; o_{-}, o_{+}\right)$produces at least a real two-dimensional family which contradicts the index hypothesis. (See $[\mathbf{F 1 3}, \mathbf{F H S}]$ for the semi-positive case and [FOn, LT1] in general.)

On the other hand, if $A=0$, any $J_{0}$-holomorphic sphere must be constant and so the corresponding configuration $\left(\chi_{-}\right.$, const, $\left.\chi_{+}\right)$becomes a full gradient trajectory $\chi=\chi_{-} \# \chi_{+}$. Unless $\chi$ is constant, i.e., unless $p=q$, $\mathbb{R}$-translation produces at least one-dimensional family which again contradicts to the index hypothesis. This finishes the proof.

Now we are ready to finish the proof of the identity

$$
\Phi \circ \Psi-\mathrm{i} d=h_{\mathrm{pss}}^{\Phi \Psi} \partial_{\left(f, J_{0}\right)}^{\mathrm{Mors}}+\partial_{\left(f, J_{0}\right)}^{\mathrm{Mors}} h_{\mathrm{pss}}^{\Phi \Psi} .
$$

A priori, Proposition 16.1 only implies

$$
\sum_{q, A}\langle(\Phi \circ \Psi-\mathrm{i} d)(p), q \#(-A)\rangle=\sum_{q, A}\left\langle h_{\mathrm{pss}}^{\Phi \Psi} \partial_{\left(f, J_{0}\right)}^{\mathrm{Morse}}(p)+\partial_{\left(f, J_{0}\right)}^{\mathrm{Morse}} h_{\mathrm{pss}}^{\Phi \Psi}(p), q \#(-A)\right\rangle .
$$

But the above lemma implies

$$
\langle(p), q \#(-A)\rangle=0
$$

unless $A=0$ and $p=q$. This finishes the proof of (16.2). 


\section{Acknowledgments}

Y.-G. Oh is partially supported by the NSF grant \# DMS 0503954 \& 0904197.

Y.-G. Oh would like to thank K. Fukaya, H. Ohta and K. Ono for the collaboration of the book [FOOO2]. A large part of analysis carried out in Part II of the current work is much influenced by the scale-dependent gluing analysis given in Chapter 10 of the book. He also thanks Bumsig Kim for the help in the argument used in the proof of Proposition 6.1, and National Institute for Mathematical Sciences (NIMS) in Korea for providing its financial support and office space during the fall of 2009 where the final version of the present paper is finished. K. Zhu would like to thank the math department of University of Wisconsin-Madison and Korea Institute of Advanced Study for the nice environment, where most of his research on this paper was carried out.

Finally, but not the least, we would like to express our deep gratitude to the anonymous referee for pointing out many inaccuracies in our presentation and providing many suggestions to improve the presentation of the paper.

\section{References}

[Ba] A. Banyaga, Sur la structure du groupe des difféomorphismes qui préservent une forme symplectique, Comm. Math. Helv. 53 (1978), 174-227.

[BC] P. Biran and O. Cornea, A Lagrangian quantum homology, in "New Perspectives and Challenges in Symplectic Field Theory", CRM Proc. and Lecture Notes, 49, 2009.

[Bou] F. Bourgeois, A Morse-Bott approach to contact homology, PhD Dissertation, Standford University, 2002 (the version 2006).

[BEHWZ] F. Bourgeois, Y. Eliashberg, H. Hofer, K. Wysocki and E. Zehnder, Compactness results in symplectic field theory, Geom. Topol. 7 (2003), 799-888, arXiv: math.SG/0308183.

[CZ] C. C. Conley and E. Zehnder, Morse-type index theory for flows and periodic solutions of Hamiltonian equations, Comm. Pure Appl. Math. 37 (1984), 207-253.

[CL] O. Cornea and F. Lalonde, Cluster homology: an overwiev of the construction and results, ERA - AMS 12 (2006), 1-12.

[D] S. Donaldson, An application of gauge theory to the topology of 4-manifolds, J. Differ. Geom. 18 (1983), 269-316.

[Ek] T. Ekholm, Morse flow trees and Legendrian contact homology in 1-jet spaces, Geometry Topology 11 (2007), 1083-1224, arXiv: math.SG/0509386.

[EGH] Y. Eliashberg, A. Givental and H. Hofer, Introduction to symplectic field theory, Geom. Func. Anal. Special Volume (2000), 560-673, arXiv: math.SG/0010059.

[Fl1] A. Floer, Morse theory for Lagrangian intersections, J. Differ. Geom. 28 (1988), 513-547. 
[Fl2] A. Floer, Witten's complex and infinite dimensional Morse theory, J. Differ. Geom. 30 (1989), 207-221.

[Fl3] A. Floer, Symplectic fixed points and holomorphic spheres, Commun. Math. Phys. 120 (1989), 575-611.

[FHS] A. Floer, H. Hofer and D. Salamon, Transversality in elliptic Morse theory for the symplectic action, Duke Math. J. 80 (1995), 251-292.

[Fu2] K. Fukaya, Morse homotopy and its quantization, in "Geometric Topology", (Athens, GA, 1993), AMS/IP Stud. Adv. Math., 2.1, Amer. Math. Soc., Providence, RI, 1997, 409-440.

[FOh] K. Fukaya and Y.-G. Oh, Zero-loop open strings in the cotangent bundle and Morse homotopy, Asian J. Math. 1 (1997), 96-180.

[FOOO1] K. Fukaya, Y.-G. Oh, H. Ohta and K. Ono, Lagrangian intersection Floer theory-anomaly and obstruction, in "AMS/IP Studies in Advanced Mathematics", 46, Amer. Math. Soc./International Press, 2009.

[FOOO2] K. Fukaya, Y.-G. Oh, H. Ohta and K. Ono, Lagrangian surgery and metamorphosis of pseudo-holomorphic polygons, preprint, 2009; available at http://www.math.kyoto-u.ac.jp/fukaya/fukaya.html.

[FOn] K. Fukaya and K. Ono, Arnold conjecture and Gromov-Witten invariants, Topology 38 (1999), 933-1048.

[Ha] R. Hartshorne, Algebraic geometry, GTM 52, Springer Verlag, New York, 1977.

[Hir] W. Hirsch, Differential topology, GTM 33, Springer Verlag, New York, 1997.

[Ho] H. Hofer, Pseudoholomorphic curves in symplectizations with applications to the Weinstein conjecture in dimension three, Invent. Math. 114 (1993), 515-563.

[HS] H. Hofer and D. Salamon, Floer homology and Novikov rings, in the Floer Memorial Volume, (H. Hofer, et al. eds.), Birkhaüser, 1995, 483-524.

[HV] H. Hofer and C. Viterbo, The Weinstein conjecture in the presence of holomorphic spheres, Comm. Pure Appl. Math. 45 (1992), 583-622.

[HWZ1] H. Hofer, K. Wysocki and E. Zehnder, Properties of pseudo-holomorphic curves in symplectizations I : asymptotics, Ann. Inst. H. Poincaré 13 (1996), 337-371.

[HWZ2] H. Hofer, K. Wysocki and E. Zehnder, Properties of pseudo-holomorphic curves in symplectizations $I V$ : asymptotics with degeneracies, in "Contact and Symplectic Geometry", (Cambridge, 1994), Publ. Newton 2nst, Cambridge University Press, Cambridge, England, 1996, 78-117.

[HWZ3] H. Hofer, K. Wysocki and E. Zehnder, Properties of pseudo-holomorphic curves in symplectizations III: Fredholm theory, in "Progress in Nonlinear Differential Equations and Their Applications", 35, Birkäuser, Basel/Switzerland, 1999.

[HWZ4] H. Hofer, K. Wysocki and E. Zehnder, Finite energy cylinder of small area, Ergodic Theory and Dynam. System 22 (2002), 1451-1486.

[HuT] M. Hutchings and C.H. Taubes, Gluing pseudo-holomorphic curves along branched covered cylinders II, J. Symplectic Geom. 7(1) (2009), 29-133. arXiv: math.SG/0705.2074.

[LT1] G. Liu and G. Tian, Floer homology and Arnold conjecture, J. Diff. Geom. 49 (1998), 1-74. 
[Lu] G. C. Lu, An explicit isomorphism between Floer homology and quantum homology, Pacific J. Math. 213(2) (2004), 319-363.

[MS] D. McDuff and D. Salamon, J-holomorpic curves and symplectic topology, Colloquim Publications, 52, AMS, Providence RI, 2004.

[MT] I. Mundet i Riera and G. Tian, A compactification of the moduli space of twisted holomorphic maps, Advanced Math. 222 (2009), 1117-1196.

[Oh1] Y.-G. Oh, Relative Floer and quantum cohomology and the symplectic topology of Lagrangian submanifolds, in "Contact and Symplectic Geometry", (C. B. Thomas eds.), Cambridge University Press., Cambridge UK, 1996, 201-267.

[Oh2] Y.-G. Oh, Construction of spectral invariants of Hamiltonian paths on closed symplectic manifolds, in "The Breadth of symplectic and Poisson geometry", Prog. Math. 232, Birkhäuser, Boston, 2005, 525-570, arXiv: math.SG/0405064.

[Oh3] Y.-G. Oh, Spectral invariants, analysis of the Floer moduli space and geometry of Hamiltonian diffeomorphisms, Duke Math. J. 130 (2005), 199-295, arXiv: math.SG/0403083.

[Oh4] Y.-G. Oh, Lectures on Floer theory and spectral invariants of Hamiltonian flows, in "Morse Theoretic Methosd in Nonlinear Analysis and in Symplectic Topology", Springer, 2006, 321-416.

[Oh5] Y.-G. Oh, An existence theorem, with energy bounds, of Floer's perturbed Cauchy-Riemann equation with jumping discontinuity, preprint, 2002, arXiv: math.SG/0207214.

[OZ1] Y.-G. Oh and K. Zhu, Embedding property of J-holomorphic curves in CalabiYau manifolds for generic J, Asian J. Math. 13 (2009), 323-340.

[OZ2] Y.-G. Oh and K. Zhu, Thick-thin decomposition of Floer trajectories and adiabatic gluing, preprint 2011, arXiv: 1103.3525.

[PSS] S. Piunikhin, D. Salamon and M. Schwarz, Symplectic Floer-Donaldson theory and quantum cohomology, Publ. Newton. Inst. 8, (C. B. Thomas ed.), Cambridge University Press, Cambridge, UK, 1996, 171-200.

[R] W. Ruan, The Fukaya category of symplectic neighborhood of a non-Hausdorff manifold, arXiv: math/SG/0602119

[SW] D. Salamon, J. Weber, Floer homology and the heat flow, GAFA 16 (2006), 1050-1138.

[SZ] D. Salamon and E. Zehnder, Morse theory for periodic solutions of Hamiltonian systems and the Maslov index, Comm. Pure Appl. Math. 45 (1992), 1303-1360.

[Sc1] M. Schwarz, A quantum cup-length estimate for symplectic fixed points, Invent. Math. 133 (1998), 353-397.

[Sc2] M. Schwarz, On the action spectrum for closed symplectically aspherical manifolds, Pacific J. Math. 193 (2000), 419-461.

[Se1] P. Seidel, $\pi_{1}$ of symplectic diffeomorphism groups and invertibles in quantum homology rings, GAFA 7 (1997), 1046-1095.

[Se2] P. Seidel, A long exact sequence for symplectic Floer cohomology, Topology 42 (2003), 1003-1063. 
[Se3] P. Seidel, Fukaya categories and Picard-Lefschetz theory, in "Zürich Lectures in Advanced Math. European Mathematical Society (EMS)", Zürich, 2008.

[Si] J.-C. Sikorav, Some properties of holomorpic curves in almost complex manifolds, in "Holomorphic Curves in Symplectic Geometry", (M. Audin and Lafontaine, J. ed.), Birkhäuser, Basel, 1994, 165-189.

[Ve] I. N. Vekua, Generalized analytic functions, Pergamon, London, 1962.

[We1] A. Weinstein, On extending the Conley-Zehnder fixed point theorem to other manifolds, in "Nonlinear Functional Analysis and Its Applications, Part 2", (Calif. Berkeley, 1983), Proc. Symp. Pure Math., 45, Part 2, Amer. Math. Soc., Providence, RI, 1986, 541-544.

[We2] A. Weinstein, Blowing up realizations of Heisenberg-Poisson manifolds, Bull. Soc. Math. 113(2) (1989), 381-406.

\section{Index}

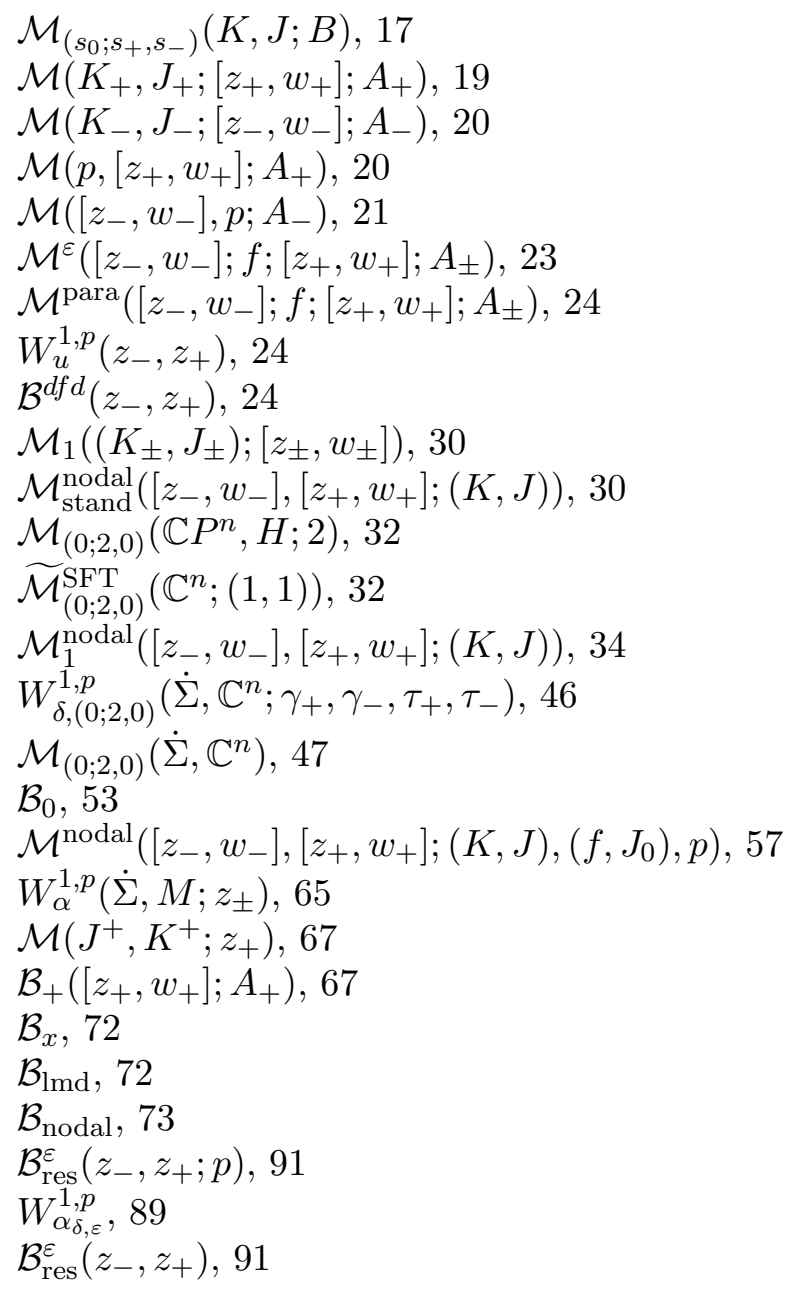


Department of Mathematics, UNIVERSITY OF WISCONSIN-MADISON,

MADISON, WI, 53706 AND

Korea Institute for Advanced Study,

Seoul, Korea

E-mail address: oh@math.wisc.edu

Department of Mathematics,

UNIVERSITY OF WISCONSIN-MADISON,

MAdison, WI 53706,

Current Address: Department of Mathematics,

University of Minnesota,

Minneapolis, MN 55455 ,

E-mail address: zhux0086@math.umn.edu

Received 04/22/2008, accepted 03/03/2011 